\author{
Dissertation zur Erlangung des Doktorgrades \\ der Fakultät für Biologie \\ der Ludwig-Maximilians-Universität München
}

Structure and Architecture of Eisosomes

\author{
vorgelegt von \\ Lena Karotki
}

2012 



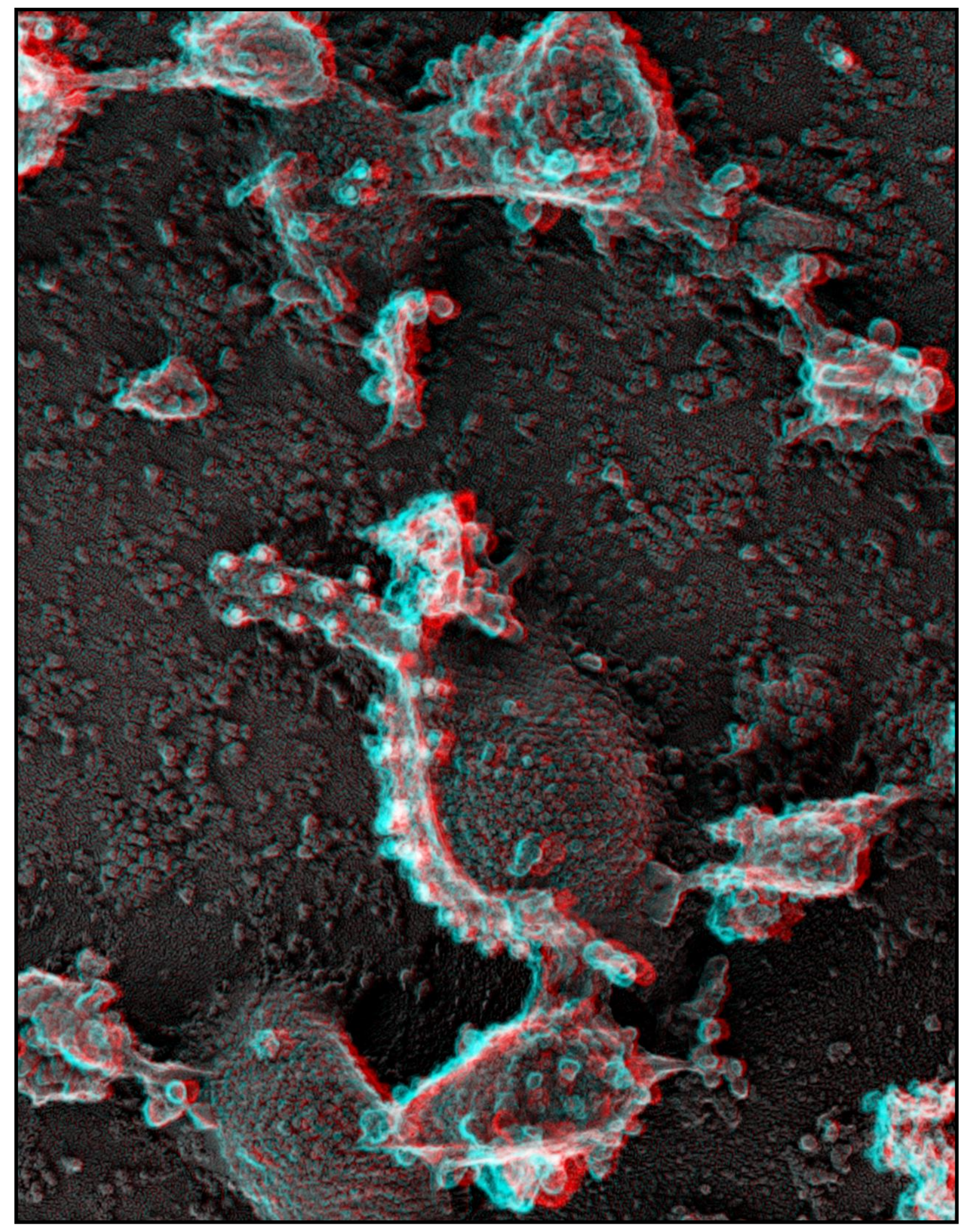




\section{Eidesstattliche Versicherung}

Ich versichere hiermit ehrenwörtlich, dass die vorgelegte Dissertation von mir selbstständig und ohne unerlaubte Hilfe angefertigt ist.

München, den

(Unterschrift)

\section{Erklärung}

Hiermit erkäre ich,

$\square$ dass die Dissertation nicht ganz oder in wesentlichen Teilen einer anderen Prüfungskomission vorgelegt worden ist

$\square$ dass ich mich anderweitig einer Doktorprüfung ohne Erfolg nicht unterzogen habe

München, den

(Unterschrift)

Diese Dissertation wurde von Prof. Dr. Stefan Jentsch betreut. Die Dissertation wurde eingereicht am

1. Gutachter: Prof. Dr. Stefan Jentsch

2. Gutachter: Prof. Dr. Charles David

Tag der mündlichen Prüfung: 03.09.2012 


\section{Table of Contents}

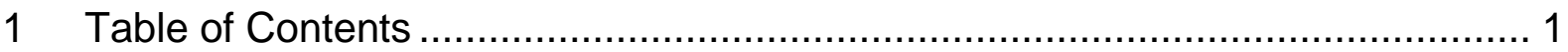

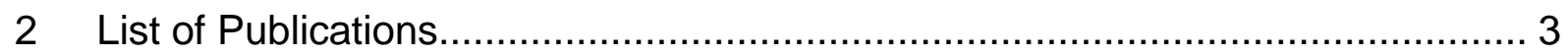

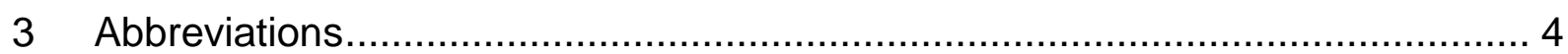

4 Summary

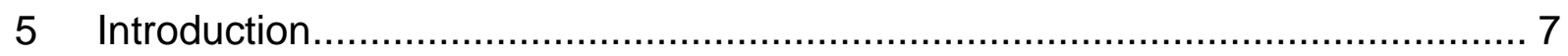

5.1 The composition of the plasma membrane …...................................... 7

5.1.1 The fluid mosaic model ........................................................ 7

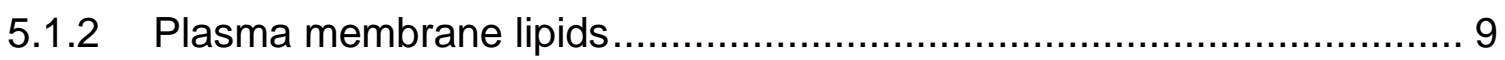

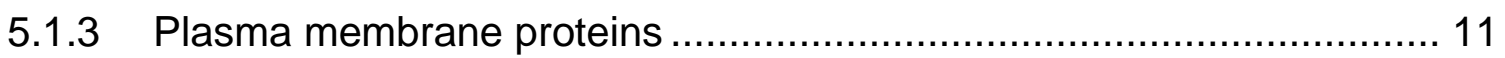

5.2 Plasma membrane organization ....................................................... 13

5.2.1 Macrodomain organization of the plasma membrane ........................ 13

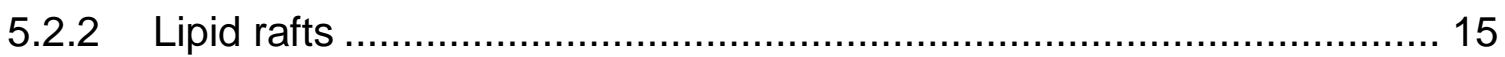

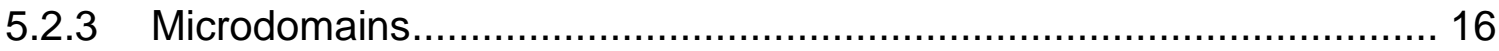

5.3 Structural aspects of membrane domains .......................................... 18

5.3.1 Lipid packing and asymmetry as driving force for membrane shaping.. 19

5.3.2 Membrane shaping by proteins ................................................. 20

5.3.3 Membrane deformation by BAR domain containing proteins ............... 21

5.4 Plasma membrane organization in yeast Saccharomyces cerevisiae ......... 26

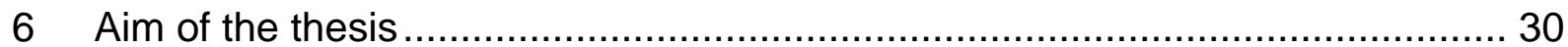

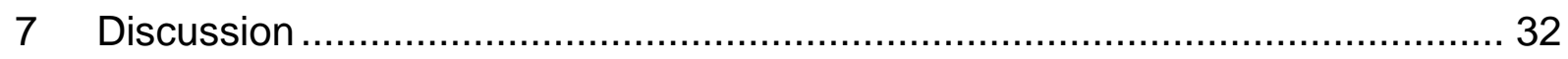

7.1 Pil1 and Lsp1 assemble into large multimeric complexes ........................ 32

7.2 Pil1 and Lsp1 bind phosphoinositides ……........................................... 36 
7.3 Pil1 and Lsp1 sculpt membranes....................................................... 39

7.4 Pil1 and Lsp1 stabilize plasma membrane domains ............................... 44

7.5 Evolutionary conservation of eisosomes ................................................. 45

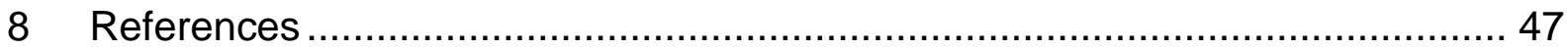

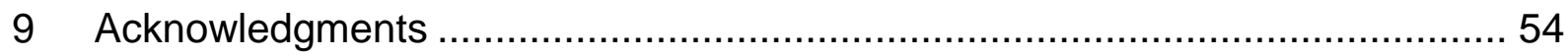

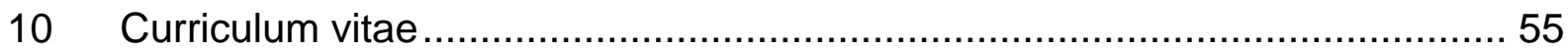

11 Declaration of Individual Contributions …................................................ 57

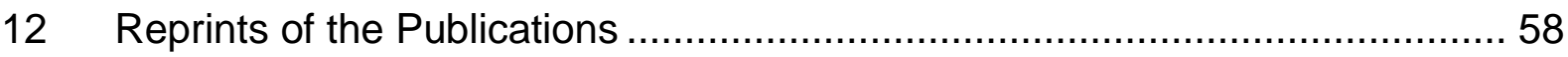




\section{List of Publications}

\section{Publication 1:}

Wang H, Kakaradov B, Collins SR, Karotki L, Fiedler D, Shales M, Shokat KM, Walther TC, Krogan NJ, and Koller D. (2009). A complex-based reconstruction of the Saccharomyces cerevisiae interactome. Mol Cell Proteomics 8, 1361-1381

\section{Publication 2:}

Ziolkowska NE, Karotki L, Rehman M, Huiskonen JT, and Walther TC (2011). Eisosome-driven plasma membrane organization is mediated by BAR domains. Nat Struct Mol Biol 18, 854-856.

\section{Publication 3:}

Karotki L, Huiskonen JT, Stefan JS, Ziolkowska NE, Roth R, Surma MA, Krogan NJ, Emr SD, Heuser J, Grünewald K, Walther TC (2011). Eisosome Proteins Assemble into a Membrane Scaffold. J Cell Biol 195, 889-902. 


\section{Abbreviations}

3D

three dimensional

BAR

Bin, Amphiphysin, ㅌvs161/167

CCP

clathrin coated pit

$\mathrm{CCV}$

clathrin-coated vesicles

COP

coatomer protein

cryo-ET

cryo-electron tomography

DAG

Diacylglycerol

DEEM

deep-etch electron microscopy

EM

electron microscopy

ER

endoplasmatic reticulum

$\mathrm{FCH}$

FES-CIP4 homology

F-BAR

FCH-BAR

GFP

green fluorescent protein

GPI

glycosylphosphatidylinositol

GUV

giant unilamellar vesicle

I-BAR

Inverse-BAR

IPC

inositol phosphate ceramide

$I_{d}$

liquid-disordered

$\mathrm{I}_{0}$

liquid-ordered

LPC

lysophosphatidylcholine

$\mathrm{M}\left(\mathrm{IP}_{2}\right) \mathrm{C}$

mannosyl-diinositolphosphate-ceramide

MCC

membrane compartment containing Can1 


\begin{tabular}{|c|c|}
\hline MCP & membrane compartment containing Pma1 \\
\hline MCT & membrane compartment of TORC2 \\
\hline MDCK & Madin-Darby canine kidney \\
\hline MIPC & mannosyl-inositolphosphate-ceramide \\
\hline NBD & nitrobenzoxadizole \\
\hline PA & phosphatidic acid \\
\hline PC & phosphatidylcholine \\
\hline PE & phosphatidylethanolamine \\
\hline PH domain & Pleckstrin homology domain \\
\hline $\mathrm{Pl}$ & phosphatidylinositol \\
\hline $\mathrm{PI}(3) \mathrm{P}$ & phosphoinositol-3-phosphate \\
\hline $\mathrm{PI}(4) \mathrm{P}$ & phosphoinositol-4-phosphate \\
\hline $\mathrm{PI}(4,5) \mathrm{P}_{2}$ & phosphoinositol-4,5-bisphosphate \\
\hline PS & phosphatidylserine \\
\hline S.cerevisiae & Saccharomyces cerevisiae \\
\hline SM & sphingomyelin \\
\hline SNXs & sorting nexins \\
\hline SPT & single particle tracking \\
\hline TGN & trans-Golgi network \\
\hline TORC2 & target of rapamycin complex 2 \\
\hline
\end{tabular}




\section{Summary}

The plasma membrane is a fundamental feature of life, since it constitutes the boundary between the cell and its environment. To coordinate the various different reactions occuring there, it is highly dynamic and its constituents are organized into domains of distinct protein and lipid composition. This lateral compartmentalization is at least partially mediated by huge protein complexes, termed eisosomes, which localize in a uniform punctuate pattern at the plasma membrane and are mainly composed of two proteins, Pil1 and Lsp1. Essential questions towards an understanding of eisosome-mediated plasma membane organization refer to their molecular architecture as well as to the mechanism mediating the lateral segregation of proteins and lipids into distinct compartments. In order to answer these questions, the work of my thesis characterizes the structure and architecture of eisosomes at several levels of resolution in vitro and in vivo. Using a combination of different biochemical and electron microscopy (EM) approaches, I show that eisosome proteins Pil1 and Lsp1 self-assemble into higher order structures, such as thin filaments and helices. Furthermore, both proteins Pil1 and Lsp1 directly bind and deform preferentially $\mathrm{PI}(4,5) \mathrm{P}_{2}$-containing membranes into long tubules. By fitting of the crystal structure of a stable core domain of Lsp1 (named "Lsp1 ASIA") into 3D models of eisosome proteins bound to membranes, I unveil the mechanism how these proteins self-assemble and bind to membranes and pinpoint amino acids that are essential in this process. Using these EM-derived 3D models of Pil1 and Lsp1, I demonstrate that these structures resemble eisosomes in vivo, From these data, I introduce a model, explaining how eisosomes are molecularly built and how they organize the plasma membrane by self-assembly into a protein scaffold that directly binds and deforms membranes with lipid-binding-specificity. Beyond the immediate gain of knowledge, characterization of eisosomes will most likely have an impact on our understanding of self assembly systems and how they organize cellular structure, and ultimately how that is used to regulate plasma membrane organization and endocytosis. 


\section{Introduction}

\subsection{The composition of the plasma membrane}

The plasma membrane constitutes the boundary between the cell and its environment. As such, it is a fundamental feature of life and crucial for a broad variety of functions, such as cell polarity establishment, cell motility or nutrient absorption. To achieve these many tasks, the plasma membrane is highly dynamic and its composition has to be constantly remodeled according to need. Consequently, the presence of receptors, transporters or signaling molecules achieves tight regulation, by a delicate interplay of proteins and lipids within the plasma membrane.

\subsubsection{The fluid mosaic model}

In 1972 Singer and Nicolson proposed a concept for membrane organization called "fluid mosaic model", which describes biological membranes as twodimensional solutions consisting of globular proteins that freely diffuse in a phopsholipid bilayer (Singer and Nicolson, 1972). The concept is based on general thermodynamic considerations about membranes and their components and involves two kinds of non-covalent interactions, hydrophobic and hydrophilic. Hydrophobic interactions are responsible for sequestering non-polar groups away from water, while hydrophilic interactions lead to the preference of ionic and polar groups for an aqueous environment. Cellular membranes consist mostly of amphipathic phospholipids, which comprise of hydrophobic moieties, the fatty acyl chains and a hydrophilic head group, which can be differently charged. According to thermodynamics, matter prefers to maintain a low energy state, which places certain restrictions on models for membrane structure. Since hydrophobic and hydrophilic interactions have to be maximized, the two leaflets associate in a way that two phospholipid fatty acid tails face towards each other, thereby being sequestered away from contact with water. In contrast, their hydrophilic headgroups are in direct contact with the aqueous phase on the exterior surfaces (Figure 1). As such, lipid bilayers are sufficiently stabilized by non-covalent forces and do not require covalent interactions between individual molecules. The hydrophobic core of this lipid bilayer is estimated to be $\sim 30 \AA$ thick, but varies depending on the chemical composition and 

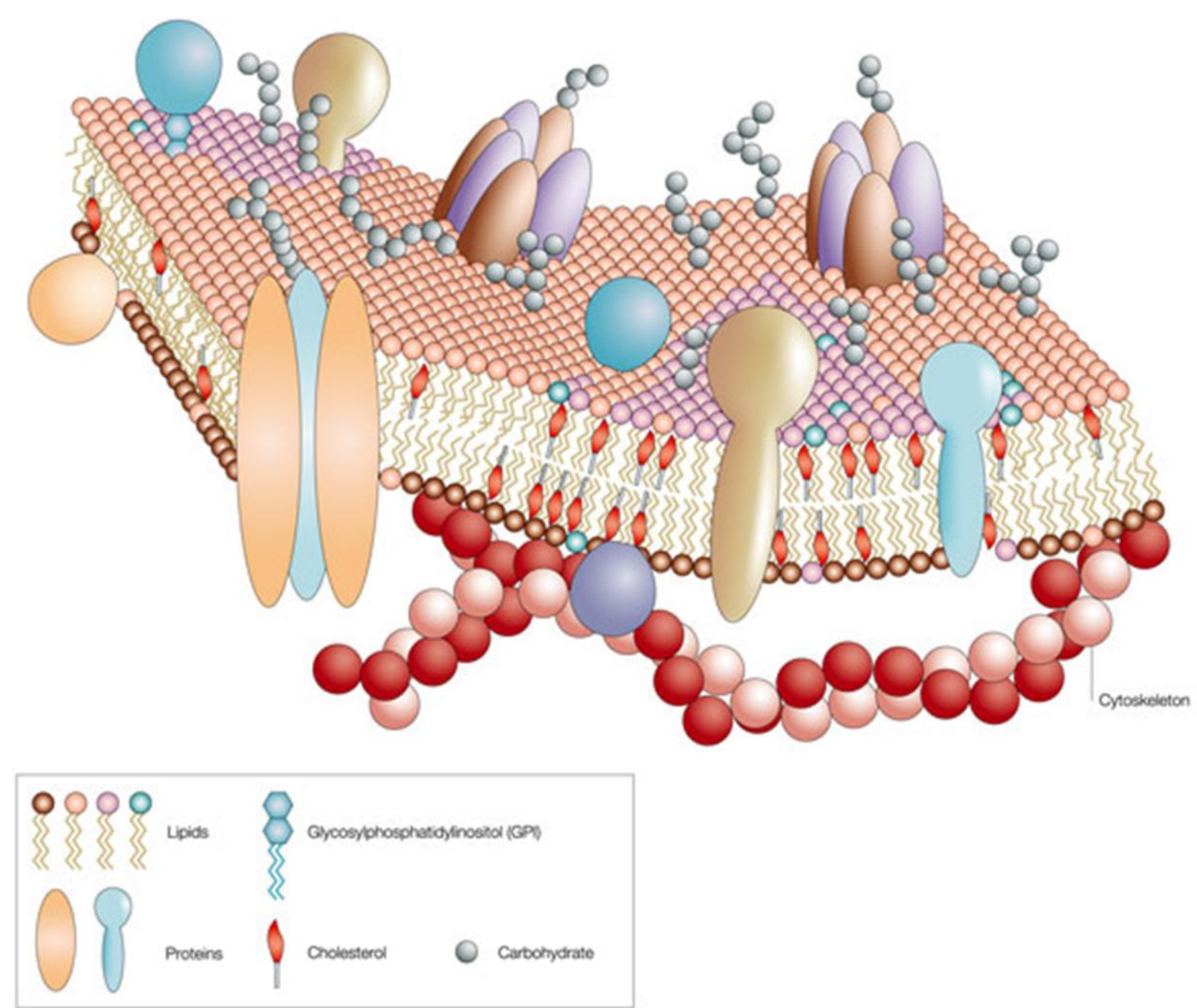

Figure 1: The fluid mosaik model of the plasma membrane (image adopted from http://www.nature.com/horizon/livingfrontier/background/figs/membrane f2.html

hydration level of the bilayer (Lee, 2003). Embedded within the hydrophobic core of the bilayer, globular proteins can diffuse laterally with their non-polar amino acids buried inside and shielded from water. Their polar and ionic groups face outwards, exposed to the surrounding extracellular space or the cytoplasm, where they can react with other molecules (Figure 1).

Although, this concept still persists as the fundamental framework for the dynamic structure of biological membranes, certain aspects of the fluid mosaic model are considerably more sophisticated in nature than originally postulated. Single particle tracking (SPT) of E-cadherin, epidermal growth factor receptor and transferrin receptor showed that $50-75 \%$ of their pool is confined to compartments of 0.04 $0.24 \mu \mathrm{m}^{2}$, showing hindrance of the free diffusion of integral membrane proteins 
(Kusumi et al., 1993). Consistent with this observation, research during the last two decades showed increasing evidence that membranes exhibit lateral heterogeneity.

Biological membranes are not passive solvents of amphipathic proteins and lipids, but rather exhibit inhomogeneous distribution. It is the unique dynamics of the multicomponent lipid bilayer that gives cell membranes their specific properties. To fully understand the complex principles behind their dynamics, requires detailed characterization of the various lipid types constituting the membrane and furthermore, their interactions with each other as well as membrane proteins, respectively.

\subsubsection{Plasma membrane lipids}

Lipids and their metabolites play an important role in regulation and control of cellular function and disease. Cells combine structural properties as well as signaling functions of lipids to orchestrate their many membrane related functions. Therefore, cellular membranes are composed of a complex mixture of different lipid types. These different lipid types vary in their chemical constitution, so that alternating compositions of constituents have an impact on the physical character of a membrane. Specifically, cellular lipids can be classified into three major classes: sterols, sphingolipids and glycerolipids (Figure 2) (van der Rest et al., 1995).

An abundant lipid species in cellular membranes is constituted by sterols. Structurally, they are compact, hydrophobic molecules, consisting of a rigid four-ring system backbone, a non-polar hydrocarbon tail and a single hydroxyl group forming the polar head group. Their presence in the plasma membrane leads to tighter lateral packing of neighboring lipids, so that plasma membrane fluidity as well as permeability for polar molecules is decreased. Based on their impact on fluidity, sterols are important regulators of biophysical membrane characteristics and could also have an impact on the lateral movement of proteins in the membrane (Daum et al., 1998).

Sphingolipids are ubiquitously present in the plasma membrane and constitute $30 \%$ of the total phospholipid content. Additionally, they play a role as second messengers for regulating signal transduction involved in cell growth control, endocytosis, actin cytoskeleton regulation and cell wall integrity (Dickson et al., 2006; van der Rest et al., 1995). Chemically, they are composed of a sphingoid long-chain 


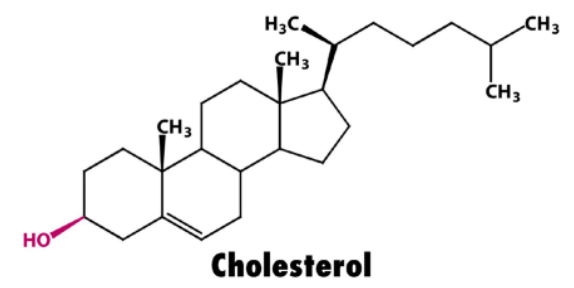<smiles>[R]C(=O)N[C@@H]1COP2(OCC[N+](C)(C)C)(OO2)O[C@@H](C)/C=C/[C@@H]1O</smiles>

Sphingomyelin<smiles>[R]C(=O)OC[C@@H](COP(C)(=O)OCC([NH3+])C([2H])=O)OC([R])=O</smiles>

Phosphatidylserine<smiles>[R]C(=O)OC[C@H](COP(C)(=O)OCC[NH3+])OC([R])=O</smiles>

Phosphatidylethanolamine<smiles>[R]C(=O)OCC(COC([R])=O)OC(=O)OCC[N+](C)(C)C</smiles>

Phosphatidylcholine

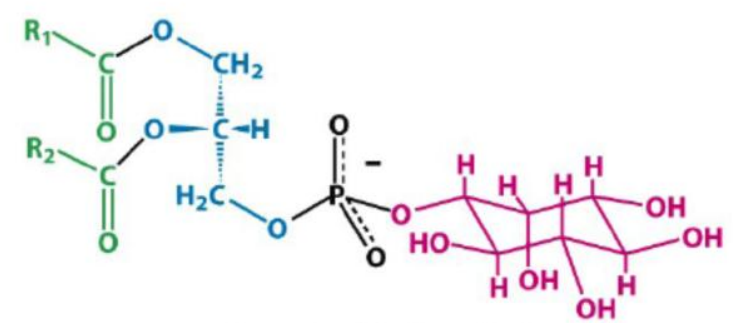

Phosphatidylinositol

Figure 2: Lipids of the plasma membrane (adopted from Biochemistry, Sixth Edition, 2007)

base (LCB), a fatty acid and a polar head group. Yeast for example uses phytosphingosine that is amide-linked to a hydroxyl $\mathrm{C}_{26}$ fatty acid to form a ceramide, to which additional headgroups are added. The resulting major sphingolipid types are inositol phosphate ceramide (IPC), mannosyl-inositolphosphate-ceramide (MIPC) and mannosyl-diinositolphosphate-ceramide $\left(\mathrm{M}\left(\mathrm{IP}_{2}\right) \mathrm{C}\right)$ (van der Rest et al., 1995).

The third major lipid class in biological membranes is represented by glycerophospholipids. They consist of a glycerol backbone with two esterified fatty acid chains and an inorganic phosphate. This phosphoryl group can be esterified to a variety of substituents, thereby defining the final lipid type: no substituent in 
phosphatidic acid (PA), choline in phosphatidylcholine (PC), ethanolamine in phosphatidylethanolamine (PE), serine in phosphatidylserine (PS) or myo-inositol in phosphatidylinositol (PI) (van der Rest et al., 1995). The inositol ring of PI can furthermore be subject to covalent modification by PI kinases and phosphatases, attaching and removing phosphate groups. $\mathrm{PI}$ and its phosphorylated derivatives are key regulators of cell growth and survival, membrane trafficking and cytoskeletal dynamics (Di Paolo and De Camilli, 2006; Hurley, 2006; Lemmon, 2008; Roth, 2004). Specific PI derivatives can be considered as organelle marker, that can be targeted to individual intracellular compartments by recognition of the unique arrangement of phosphate groups around the inositol ring: for example the plasma membrane is enriched in phosphoinositol-4,5-bisphosphate $\left(\mathrm{PI}(4,5) \mathrm{P}_{2}\right)$, whereas phosphoinositol4-phosphate $(\mathrm{Pl}(4) \mathrm{P})$ and phosphoinositol-3-phosphate $(\mathrm{PI}(3) \mathrm{P})$ are primarily in the Golgi and early endosomes, respectively (Kutateladze, 2010).

\subsubsection{Plasma membrane proteins}

The mass ratio of proteins to lipids in the plasma membrane depends on the specific cell type and function, but is on average 1:1. In general, membrane proteins can be classified into two major categories: integral (intrinsic) and peripheral (extrinsic) proteins, with the category being defined by the membrane-protein interaction (Figure 3).

Integral membrane proteins have one or more hydrophobic amino acid stretches that are located within the plasma membrane. Such membrane domains can either span the whole bilayer or just insert in between them, both by interaction of hydrophobic protein residues with the fatty acid chains of the lipids. Hydrophilic parts of the protein are either exposed to the cytoplasm, the extracellular space or both sides and can be used for signal transduction or interaction with other cytoplasmic proteins. Typical examples for such proteins are lon channels, proton pumps or Gprotein coupled receptors.

In contrast, peripheral membrane proteins do not interact with the hydrophobic core of the phospholipid bilayer, but are rather attached to the membrane by a combination of hydrophobic, electrostatic or other non-covalent interactions. Such Interactions include either the binding to integral membrane proteins or a direct electrostatic interaction with the polar head groups of membrane lipids. Additionally, 


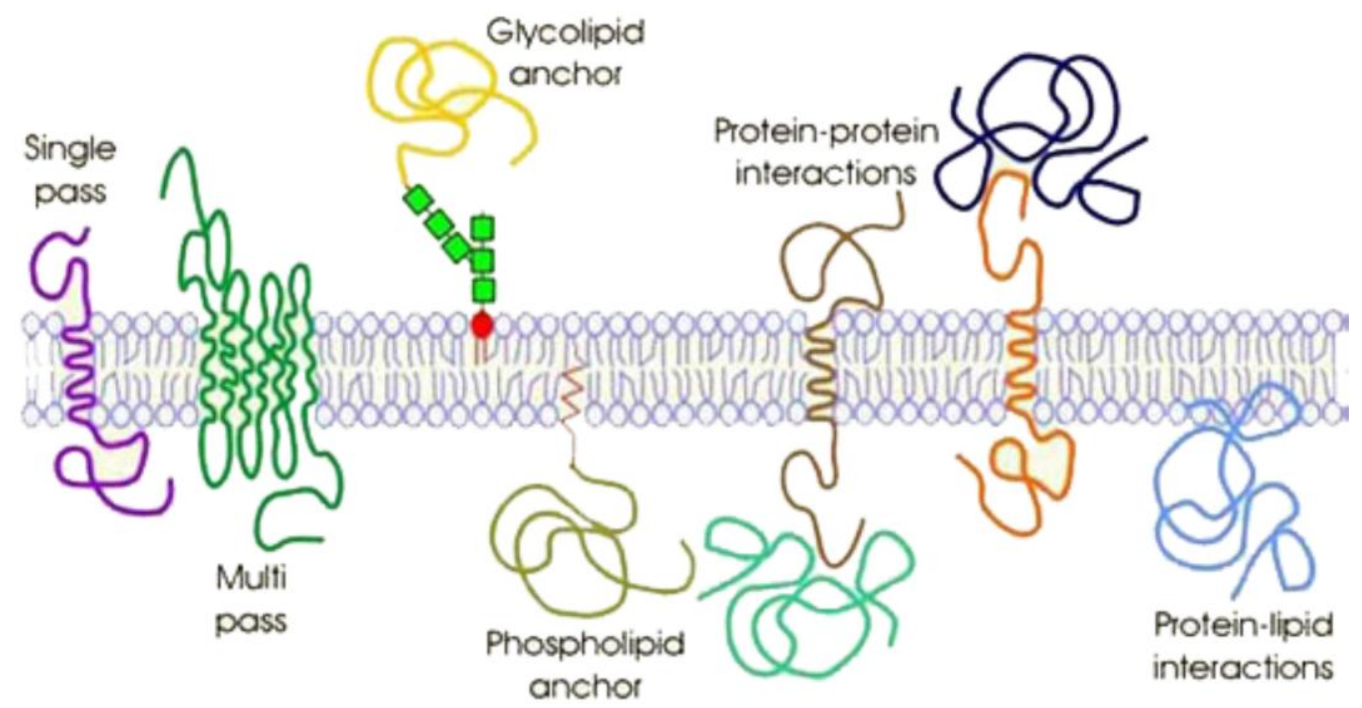

Figure 3: Membrane proteins associate with membranes in different ways.

Membrane proteins can contain lipid-spanning domains (single or multipass) or be anchored by lipid-modifications. Additionally, cytoplasmic proteins can associate with the membrane directly or embedded proteins from the inner or outer side of the membrane (adopted from http://www.utm.utoronto.ca).

fatty acid acyl chains, such as glycosylphosphatidylinositol (GPI) function as anchors in the plasma membrane, to covalently attach proteins by palmitoylation, myristoylation or penylation (Levental et al., 2010). Such lipid modifications regulate function and cellular localization of G-proteins. One example is palmitoylation of members of the Src family of tyrosine kinases, which is required for localization to specific plasma membrane domains, termed caveolae (Lisanti et al., 1994; Sargiacomo et al., 1993; Shenoy-Scaria et al., 1994). As an alternative approach to GPI anchors, proteins comprise defined membrane-targeting domains that interact with specific lipids in the membrane. Among them, the pleckstrin homology $(\mathrm{PH})$ domain was the first such domain found to associate specifically with $\operatorname{PI}(4,5) \mathrm{P}_{2}$ (Harlan et al., 1994; Haslam et al., 1993; Mayer et al., 1993). To date, PH domains have been identified ubiquitously, for example in cytoskeletal proteins, such as dynamin or regulators of small G-proteins, for eample Cdc24 in S. cerevisiae (Toenjes et al., 1999). Other examples of membrane-targeting domains include the PX domain, which preferentially binds to PI(3)P-enriched membranes, such as endosomes and vacuoles (Seet and Hong, 2006; Yu and Lemmon, 2001). The largest group of PX domain containing proteins comprises sorting nexins (SNXs), which are found to participate in endosomal sorting and trafficking in yeast and mammalian cells (Seet and Hong, 2006; Xu et al., 2001). 


\subsection{Plasma membrane organization}

Cellular membranes display a tremendous complexity of lipids and proteins to perform their many tasks. To simultaneously coordinate these processes, cells have the ability to laterally segregate biochemical reactions and thus to locally increase molecular components, promoting the efficiency of their performance (Lajoie et al., 2009; Lingwood and Simons, 2010). Such compartmentalization occurs on several cellular levels.

\subsubsection{Macrodomain organization of the plasma membrane}

Macrodomain organization is a fundamental feature to segregate plasma membranes into distinct reaction compartments. These reaction compartments comprise different lipid and protein composition and may vary in their morphology, features which are in close relationship to their function. Examples of such macrodomain formation include membranes of axons and dentrites in neurons, lamellipodia in fibroblasts or the growing bud in Saccharomyces cerevisiae ( $S$. cerevisiae) (Carmosino et al., 2010). However, the best-studied example so far is epithelial cells, such as Madin-Darby canine kidney (MDCK) cells, which specialize their surface into two distinct domains: the apical and the basolateral plasma membrane. These surfaces vary significantly in their function and are also biochemically distinct. While the apical membrane is enriched in sphingolipids, the basolateral domain contains mostly the glycerophospholipid PC (Simons and van Meer, 1988; van Meer, 1989). Besides their lipid composition, also different proteins are targeted to these surfaces in order to efficiently perform the required functions at the destined surface.

Generation and maintenance of such large-scale domain organization requires the presence of a highly specialized cellular machinery that regulates continuous sorting of newly synthesized components and controls their internalization (Matter, 2000; Mellman, 1996; Nelson and Yeaman, 2001). For example, apical polarized protein traffic is regulated by intrinsic protein sorting signals. According to these signals, proteins emerge from the trans-Golgi network (TGN) in different post-Golgi carriers (Guerriero et al., 2008; Jacob et al., 2003) and navigate through distinct subsets of endosomal compartments to their final destination (Hoekstra et al., 2004; 
Weisz and Rodriguez-Boulan, 2009). Additionally, proteins such as Rab GTPases are crucially involved in defining the trafficking routes of these transport carriers, e.g. Rab11a is present on apical recycling endosomes, where it modulates export to the apical membrane (Lapierre et al., 2001; Roland et al., 2007). In contrast, Rab8 and Rab10 participate in basolateral targeting (Babbey et al., 2006; Schuck et al., 2007).

Studies on the temporal coordination of vesicular transport in mammalian cells revealed a new layer of organization. Temperature shift to $20^{\circ} \mathrm{C}$ blocks vesicular delivery from the Golgi complex and leads to accumulation of fluorescently labeled sphingolipids and surface proteins in this compartment (van Meer et al., 1987). Strikingly, release of this temperature-induced block showed that trapped lipids and proteins leave this compartment together and appear at the appropriate epithelial surface with similar kinetics (Matlin and Simons, 1984; Pfeiffer et al., 1985; van Meer et al., 1987). Based on this observation, Simons and vanMeer postulated that not only proteins but also lipids are presorted in the TGN, in order to be transported collectively within the same carrier vesicles to their target membrane (Simons and van Meer, 1988). This was the biochemical basis for the so called "lipid raft" hypothesis. It posits that lateral self-organization of lipids together with proteins induces sub-compartmentalization to organize bioactivity of cellular membranes,

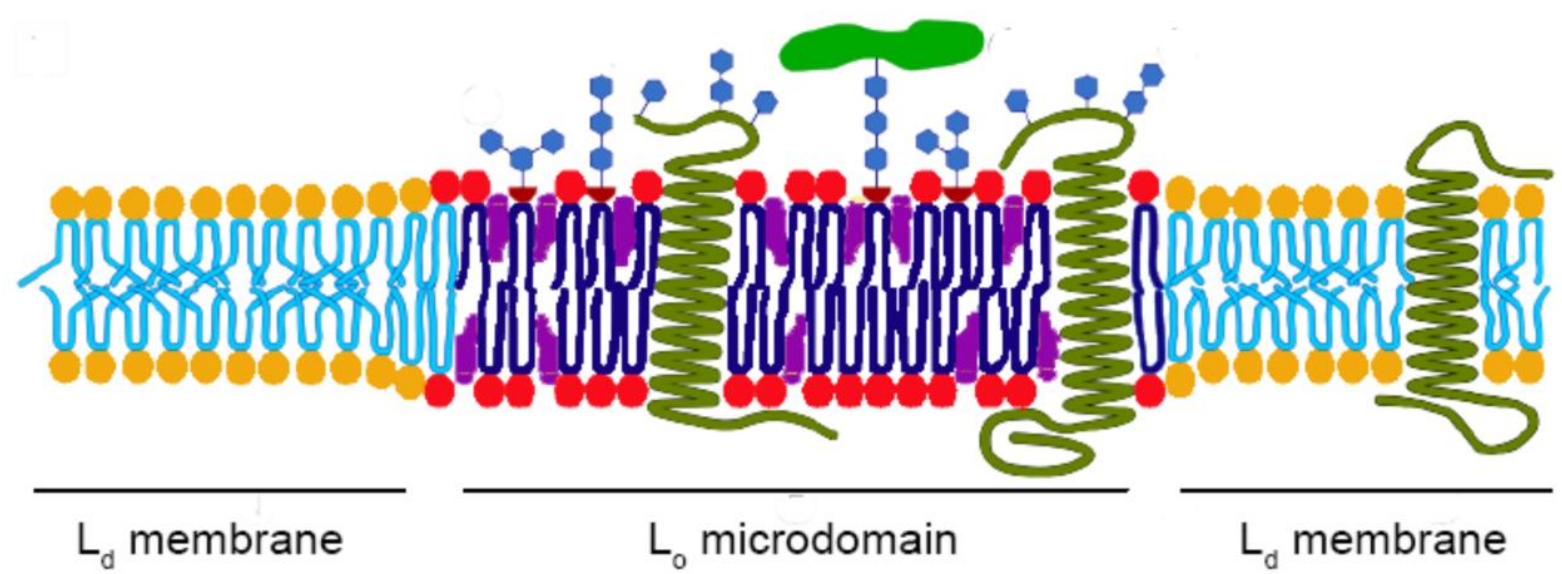

Figure 4: Schematic representation of lipid rafts in the plasma membrane (adopted from http://en.wikipedia.org/wiki/Lipid raft)

Purple cylinders represent cholesterol, which clusters together with glycosphingolipids (blue/red) forming the liquid-ordered microdomain $\left(\mathrm{I}_{0}\right)$. Lipids shown in bright blue and yellow represent liquid-disordered phases in the membrane $\left(I_{d}\right)$. $L_{0}$ microdomains also fuction as assembly platform for signaling molecules, such as e.g. transmembrane proteins (dark green) or GPI-anchored proteins (bright green). 
such as orchestrating the assembly of signaling molecules, regulating receptor and membrane protein trafficking, or influencing membrane fluidity.

\subsubsection{Lipid rafts}

Lipid rafts are thought to be the smallest units of organization in the plasma membrane (Lingwood and Simons, 2010; Munro, 2003; Simons and Vaz, 2004). Although, the biological relevance or even existence of lipid rafts has been questioned for years, current advances in technology yielded data, describing them as dynamic nanoscale assemblies or platforms, enriched in cholesterol and sphingolipids (Simons and Ikonen, 1997; Simons and Vaz, 2004). These two lipid species form a unit together, which floats in the membrane, but is more ordered and tightly packed than the surrounding phospholipid bilayer. The mechanism for lipid raft formation is based on the process of phase separation in the fluid lipid bilayer, specifically between the different lipid species according to their physical properties. Due to the rigid nature of its sterol group, cholesterol prefers interaction with hydrocarbon chains of saturated lipids, such as in sphingolipids, rather than with more bulky unsaturated lipids (Figure 4) (Simons and Vaz, 2004). Additionally, cholesterols small head group is proposed to be insufficient to shield the hydrophobic ring system from water molecules in the environment. By partitioning into the same domain, sphingolipids can provide additional shielding due to their larger head group, so that cholesterol packs among them, filling the voids between associated sphingolipids. This concept was described as the "umbrella-model" or the "condensed complex model" (Ikonen, 2008). Such phase separation can also be reconstituted in model systems, such as liqid bilayers or giant unilamellar vesicles (GUVs), showing spontaneous cholesterol-dependent lateral segregation of lipids (Ipsen et al., 1987; Kahya et al., 2003; Lentz et al., 1980).

The segregation of lipids in the membrane based on their physical properties, lead to the differentiation in cholesterol-dependent liquid-ordered $\left(I_{0}\right)$ and liquiddisordered $\left(I_{d}\right)$ phases (Figure 4) (Simons and Ikonen, 1997). Consistent with the acyl chain length of their components, $I_{0}$ phases are suggested to be thicker then $I_{d}$ phases (Harder and Simons, 1997). This morphological difference may lead to the generation of specialized plasma membrane environments, where proteins e.g. 
comprising of long transmembrane domains can preferrentially incorporate and cluster together. For example GPI-anchored proteins partition into lipid rafts in postTGN sorting vesicles and act as sorting platforms for inclusion of cargo destined for delivery to the apical membrane (Paladino et al., 2004; Simons and Ikonen, 1997). Based on this capability to segregate specific classes of lipids and proteins, rafts support lateral segregation of the plasma membrane into functional domains and for example mediate polarized delivery of proteins (Bagnat et al., 2000).

\subsubsection{Microdomains}

In vitro spontaneous phase separation leads to the formation of very large lipid phases, which is controversial to the observation of small and disperse raft domains in vivo. The reason for this discrepancy is not known yet, but might be based on the plasma membrane being close to a critical point of phase transition. Therefore, small rafts are disperse in vivo, but able to cluster upon interactions with the cytoskeleton or plasma membrane proteins (Honerkamp-Smith et al., 2008; Honerkamp-Smith et al., 2009; Veatch et al., 2008). Crosslinking-experiments of the raft ganglioside GM1 showed that lipid rafts can coalesce to form larger, micrometerscale membrane domains in a sterol-dependent manner (Lingwood et al., 2008). Interactions between lipids by themselves are weak and transient, however such microdomains can be laterally stabilized by protein-protein and/or protein-lipid interactions. An example for such microdomain organization in vivo is provided by caveolae. These are $60-80 \mathrm{~nm}$ flask-like shaped plasma membrane invaginations, which are specialized, morphologically distinguishable form of lipid rafts (Rajendran and Simons, 2005; Simons and Toomre, 2000).

Although the function of cavolae is not entirely clear, they are implicated in numerous cellular functions, such as lipid uptake and regulation, endocytosis, signal transduction and virus entry (Simons and Toomre, 2000). Consistent with their various functions, they have also been linked to disease: mutations in their molecular components have been found in breast cancer as well as in types of muscular dystrophy (Schwencke et al., 2006). Their main component is the integral membrane protein caveolin-1 (Rothberg et al., 1992), which is present with around 145 molecules per caveola (Pelkmans and Zerial, 2005) and is by itself sufficient to form these structures (Fra et al., 1995). Caveolin-1 inserts into the bilayer with its putative 


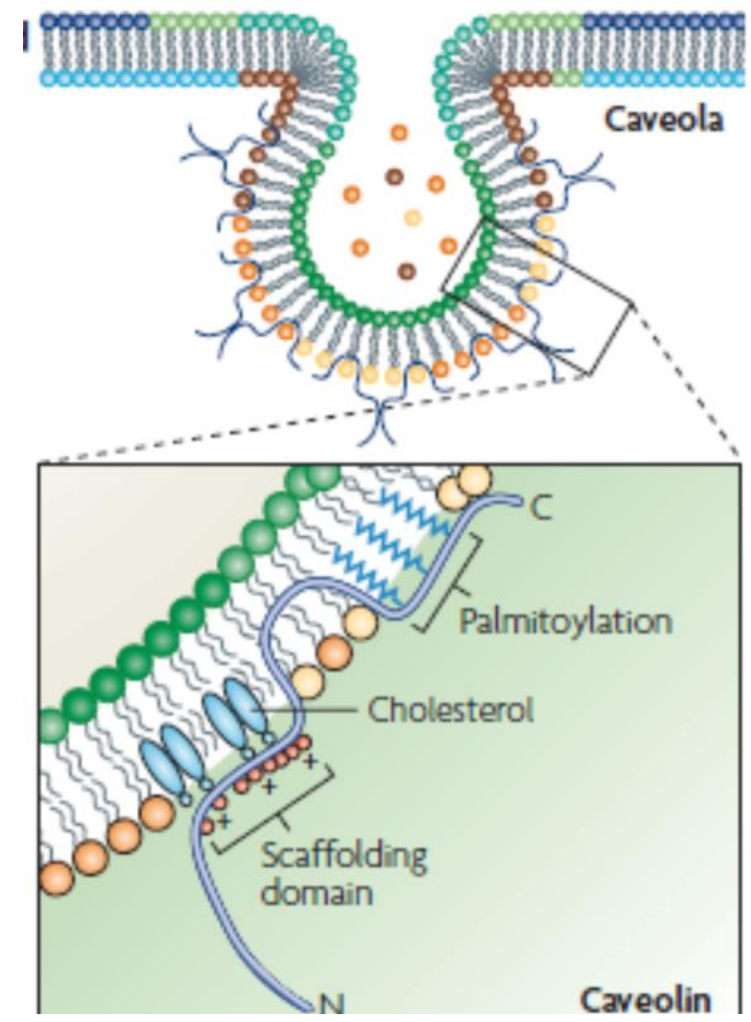

Figure 5: Structure of Caveolae and Caveolins (adopted from (Parton and Simons, 2007))

Caveolin is inserted in to the caveolar membrane with the $\mathrm{N}$ and $\mathrm{C}$ termini facing the cytoplasm and a putative 'hairpin' intramembrane domain embedded within the membrane bilayer. The scaffold domain, a highly conserved region of caveolin might have a role in cholesterol interactions through conserved basic (+) and bulky hydrophobic residues (red circles). The C terminal domain, which is close to the intramembrane domain, is modified by palmitoyl groups that insert into the lipid bilayer. The complex structures that are formed by interconnected caveolae can occupy a large area of the plasma membrane.

transmembrane hairpin domain and has strong affinity for cholesterol (Murata et al., 1995), which is enriched in lipid rafts. Sedimentation velocity experiments show that, detergent-solubilized caveolin-1 forms oligomeric structures of $340 \mathrm{kDa}$, the equivalent of 10-15 molecules. Thus, interaction of caveolin-1 with cholesterol likely triggers its oligomerization, which in turn leads to further intercalation of caveolin-1 into rafts. Based on the local increase of available cholesterol binding sites, such oligomeric building blocks subsequently fuse with each other and collect raft nanoclusters to form and stabilize them into larger domains. This is consistent with the idea of rafts being dynamic and transient structures that can change their size and composition in response to intra- and extracellular stimuli (Hancock, 2006; Sargiacomo et al., 1995; Simons and Toomre, 2000). Thus, caveolin-1 binding and recruitment of cholesterol to raft domains (Murata et al., 1995; Smart et al., 1996) could organize the lipid composition in the membrane and additionally have an impact on raft dynamics and functionality (Harder and Simons, 1997; Lajoie et al., 2009). 


\subsection{Structural aspects of membrane domains}

Membrane domain organization by local enrichment of specific lipids and proteins is often accompanied by morphological changes of the bilayer. Depending on the accumulated constituents, biological membranes can adopt a large variety of different shapes, which are usually in direct relationship to their specific function. In general, four universal types of membrane shapes can be found (Figure 6): 1) flat shapes, such as uncoated plasma membranes or non-invaginated coated pits, 2) cylindrical shapes, as in membrane carriers e.g. operating between endoplasmatic reticulum (ER) and Golgi apparatus, 3) saddle-like shapes, such as membranes connecting budding vesicles before pinching-off and 4) spherical shapes, as in coated endocytic vesicles or coatomer protein (COP)-coated vesicles (Zimmerberg and Kozlov, 2006). Molecular mechanisms to induce membrane curvature or attain the characteristic shapes of different organelles are still poorly understood. Although, all of them relate to the generation of membrane curvature (Gallop and McMahon, 2005), the processes involved are highly diverse and have specific requirements for lipid or protein involvement, as well as the physical forces that need to be applied to achieve the shape.
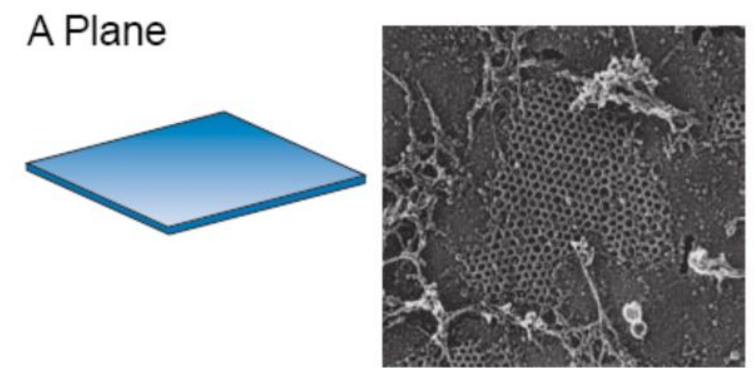

B Cylinder
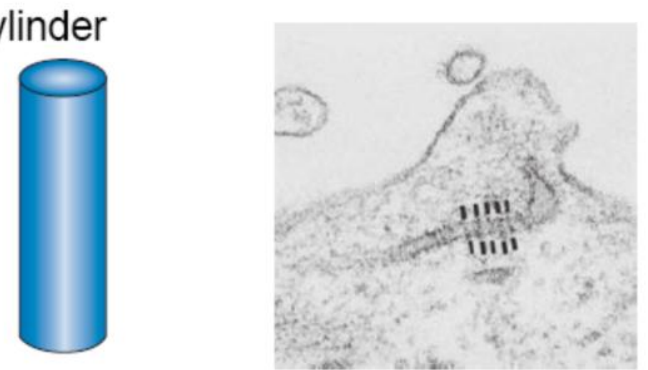

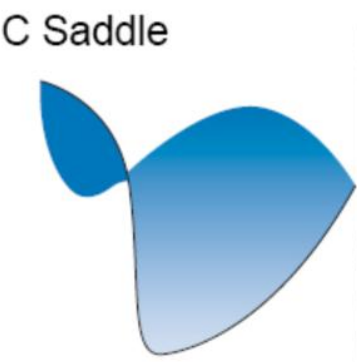

D Sphere

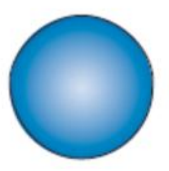

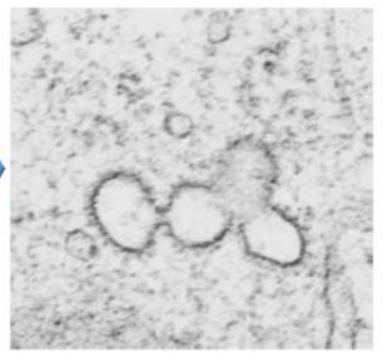

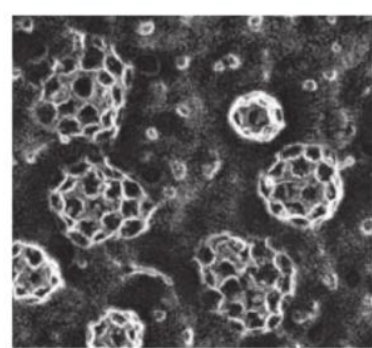

Figure 6: Basic membrane shapes (Zimmerberg and Kozlov, 2006)

(A) Flat clathrin lattice on a membrane fragment. (B) A membrane tube under a dynamin coat. (C) Neck of a membrane bud. (D) Pure clathrin cages assembled in vitro. 


\subsubsection{Lipid packing and asymmetry as driving force for membrane shaping}

The shape of cellular membranes is defined by the molecular characteristics of their constituents. Lipid molecules prefer to organize in structures that best accommodate their dynamic shape, which is not necessarily in a flat bilayer. The curvature coupling mechanism describes the two leaflets of a lipid bilayer as coupled to each other along the mid face of the membrane. This means that neither of the two leaflets can be bent individually, without the other leaflet adopting the equivalent same shape (Sheetz and Singer, 1974). Common constituents of the eukaryotic plasma membrane are PC and PS. Both lipids comprise an overall cylindrical shape and form rather flat or gently curved bilayers in vitro (Janmey and Kinnunen, 2006). Each lipid molecule with diverging shape from a cylinder has an effect on the sponatneous curvature of a membrane and local enrichment leads to asymmetric surface area distribution within the two leaflets and can thereby induce curvature in a membrane (Figure 7 adopted from (Sprong et al., 2001)). For example, lipid molecules with a large headgroup and an inverted cone shape, such as lysophospholipids, polyphosphoinositides or sphingomyelin (SM) can lead to membrane structures with positive curvature. In contrast, lipid molecules with an overall conical shape, comprise of a small hyodrophilic headgroup compared to their hydrophilic moiety, thereby inducing negative curvature. Such molecules are for example PE or diacylglycerol (DAG) .
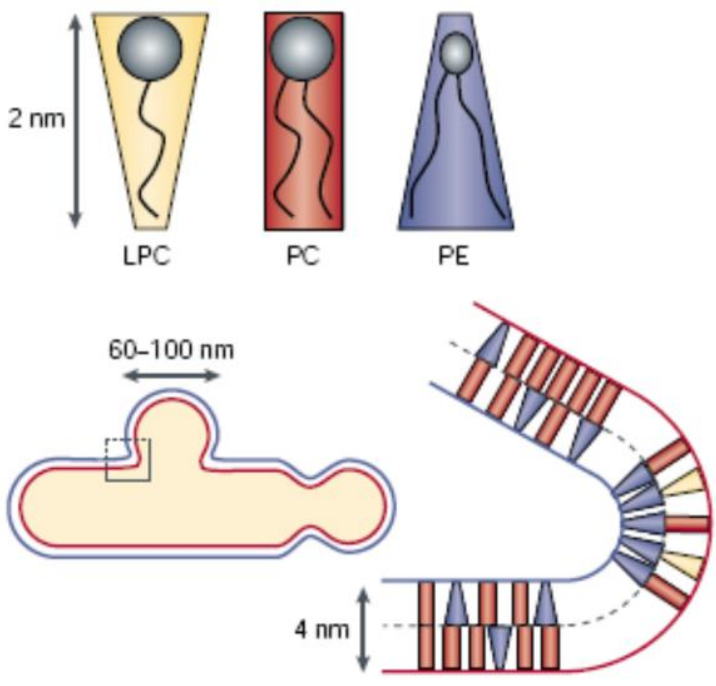

Figure 7: The molecular shape of lipids determines the physical properties of membranes (adopted from (Sprong et al., 2001)).

The overall shape of membrane lipids depends on the relative size of their fatty acid tails to polar head group. One example for a cylindrical lipid shape is PC, where the headgroup and the tails have similar sizes. Lipids with a large hydrophobic part and a relatively small headgroup such as PE, are coneshaped. In contrast, lipids with a relatively large headgroup and the fatty acid tail occupying a smaller surface area have the shape of an inverted cone, as e.g. Iysophosphatidylcholine (LPC) or SM. 
Due to the lipid composition of most cellular membranes, generation of stable high curvature shapes purely based on lipid asymmetry is energetically unfavorable (van Meer et al., 2008). Depending on the shape, this requires a monolayer area difference of $10-20 \%$ across the two layers (Zimmerberg and Kozlov, 2006). Specifically, generation of a membrane tubule requires $\approx 70 \mathrm{kcal} / \mathrm{mol}$, formation of a spherical vesicle even $\approx 300 \mathrm{kcal} / \mathrm{mol}$. Since the characteristic thermal energy of a membrane is much lower $(\approx 0.6 \mathrm{kcal} / \mathrm{mol})$, stable formation of such shapes does not occur spontaneously, but requires molecular mechanisms stabilizing such energetically unfavorable states (Shibata et al., 2009). Thus, generation and particularly stabilization of tubular or spherical shapes from cellular membranes requires a substantial and persistent amount of energy, which usually requires the aid of proteins (Zimmerberg and Kozlov, 2006).

\subsubsection{Membrane shaping by proteins}

Proteins deform membranes using two different types of forces: pulling and bending (Zimmerberg and Kozlov, 2006). Sedimentation of kinesin-coated lipid vesicles onto a network of stabilized microtubules, leads to growth of membrane

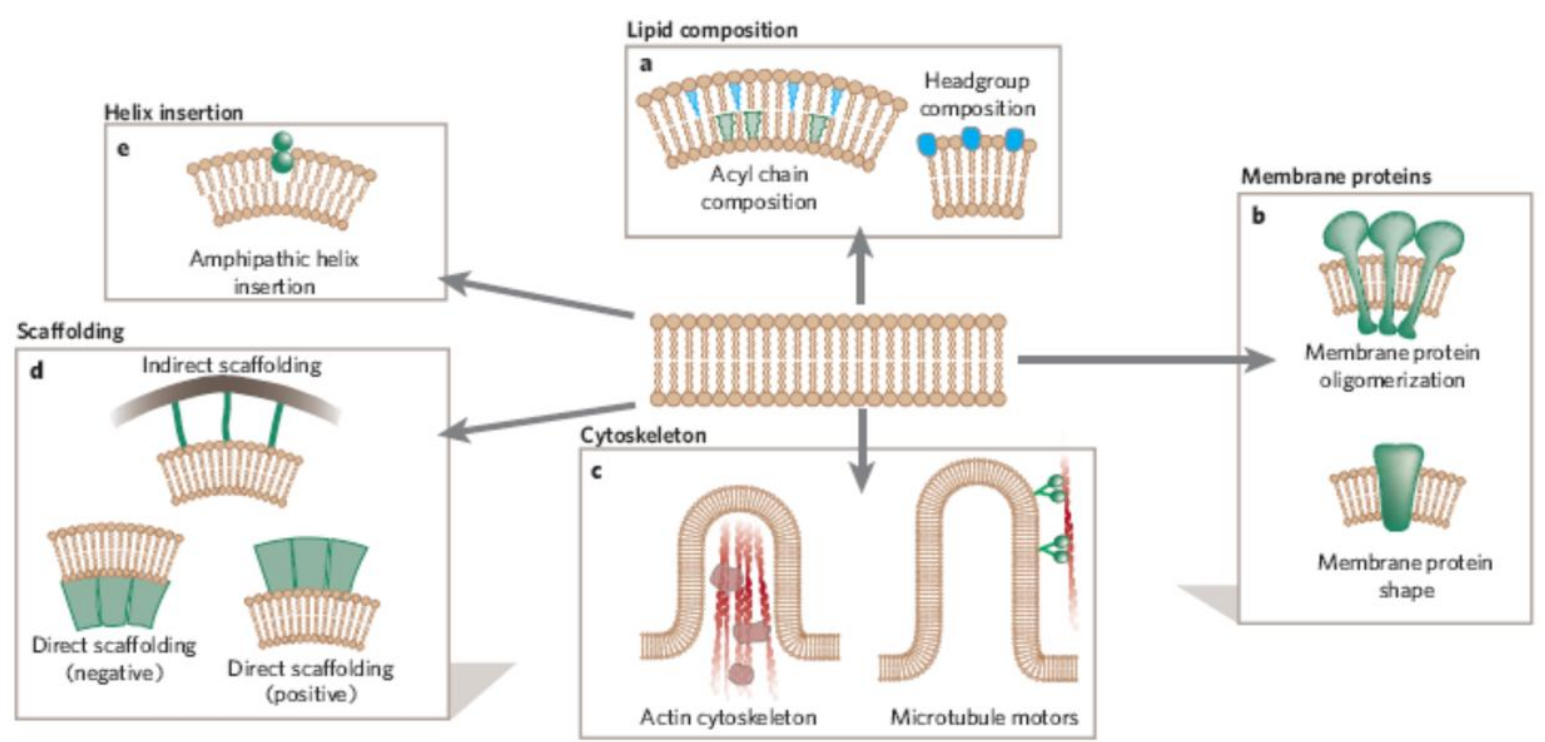

Figure 8: Mechanisms of membrane deformation (adopted from (McMahon and Gallop, 2005)). The phospholipid bilayer can be deformed causing positive or negative membrane curvature. (A) changes in lipid composition (B) influence of integral membrane proteins that have intrinsic curvature or have curvature on oligomerization (C) changes in cytoskeletal polymerization and pulling of tubules by motor proteins (D) direct and indirect scaffolding of the bilayer; e, active amphipathic helix insertion into one leaflet of the bilayer. 
tubes along the microtubule system (Koster et al., 2003; Roux et al., 2002). Together with in vivo studies, this suggested that membrane curvature can be induced by molecular motor proteins, which directly apply pulling forces onto the lipid bilayer. Alternatively, polymerization forces generated by the cytoskeleton itself have also been described to generate elaborate tubular membrane structures, a mechanism independent from motor proteins (Waterman-Storer and Salmon, 1998) (Figure 8).

Bending of a membrane can be induced by two major mechanisms, which are not mutually exclusive: scaffolding and wedging. The wedging mechanism, involves insertion of a part of the protein into the bilayer, e.g. an amphipathic helix. Perturbation in the packing of polar lipid headgroups subsequently induces membrane curvature. This mechanism has been described for a variety of proteins, examples include epsin N-terminal homology domains (Ford et al., 2002), Sar1 of the COPII coat complex (Lee et al., 2005), the reticulons of the ER membrane (Hu et al., 2008) or the ArfGAPs of the COPI coat (Drin et al., 2007). In contrast, the scaffolding mechanism insinuates that proteins apply their internal curvature to the bilayer, thereby forcing the membrane to adopt the scaffolds' shape (Peter et al., 2004). Here, usually charge interactions between the molecules and the membrane mediate curvature generation (Wang et al., 2009). The scaffolding mechanism is based on the assumption that the protein scaffold is more rigid than the membrane, which is a particularly characteristic feature of the $\underline{B}$ in, Amphiphysin, $\underline{R} v s 161 / 167$ (BAR) domain containing protein superfamily.

\subsubsection{Membrane deformation by BAR domain containing proteins}

Members of the BAR domain containing protein superfamily are described as fundamental regulators of membrane-remodeling processes throughout eukaryotes. They are recruited from the cytoplasm to participate in cellular events, e.g. formation of plasma-membrane extensions or invaginations, transport intermediates such as endocytic vesicles or the development of tubular membrane structures (Itoh et al., 2005; Lee et al., 2002; Peter et al., 2004; Tsujita et al., 2006). The molecular basis for our understanding of BAR domain containing proteins comes from crystallographic and biochemical studies (Gallop et al., 2006; Mattila et al., 2007; Peter et al., 2004). In general, all members of the family have a dimeric, bananashaped structure, formed by the antiparallel association of two monomers (Figure 9) 


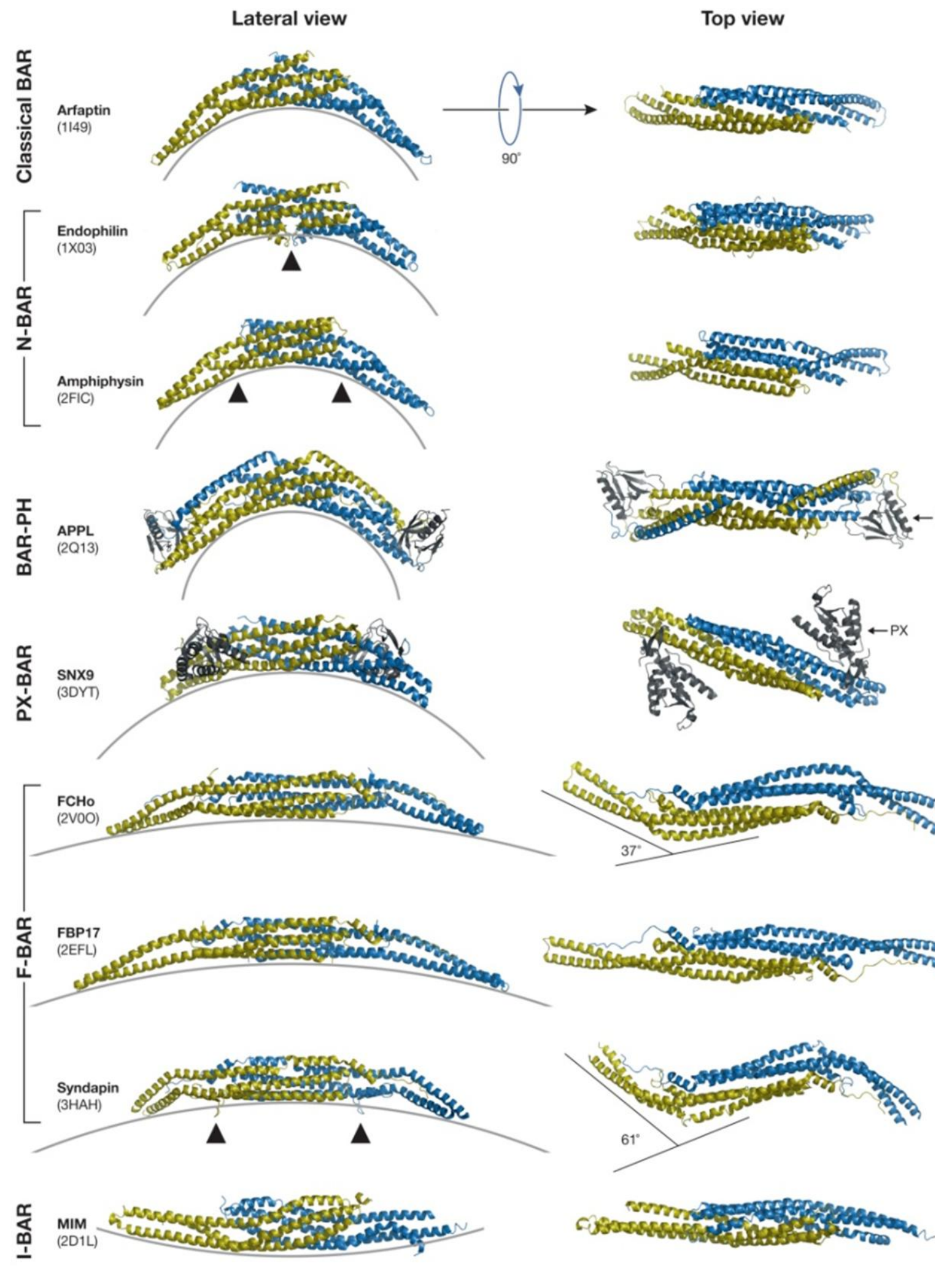

Figure 9: Comparative views of representative members of the BAR domain superfamily (adopted from (Qualmann et al., 2011)).

Each module consists of two monomers (green and turquoise). $\mathrm{PH}$ or PX domains are shown in black, amphipathic $\mathrm{N}$-termini are indicated by black arrowheads (Endophilin, Amphiphysin, Syndapin). 
(Casal et al., 2006; Masuda and Mochizuki, 2010; Shimada et al., 2007; Tarricone et al., 2001; Zhu et al., 2007). The dimer is stabilized by hydrophobic interactions between their inner surfaces, resulting in a symmetrical elongated structure with a central six-helix bundle core (3 from each monomer) and two arms (Gallop et al., 2006; Henne et al., 2007; Lee et al., 2007; Masuda and Mochizuki, 2010; Zhu et al., 2007). Based on their overall degree of curvature, they can be divided in different subgroups: the classical banana-shaped BAR domain, the more elongated crescent shaped F-BAR (FCH-BAR or EFC Extended-FCH) domain and the nearly flat zeppelin shaped I-BAR (Inverse-BAR) domain (Figure 9) (Frost et al., 2008).

Membrane binding and deformation by BAR domains is generated by interaction of positively charged amino acid patches within the concave face of the dimer with the negatively charged phospholipid headgroups in the membrane. As such, the BAR domain imposes its intrinsic curvature onto the membrane and induces curvature by applying the scaffolding mechanism (Zimmerberg and Kozlov, 2006). Consistent with this, incubation of BAR domain proteins with liposomes in vitro bends them into tubules, with the diameter matching the degree of the specific curvature of the BAR domain (Shimada et al., 2007). A variant of the classical BAR domain, the so-called N-BAR domain, additionally inserts an amphipathic helix into the membrane to enhance tubulation. Prominent members of this protein family are endophilin or amphiphysin (Gallop et al., 2006; Masuda and Mochizuki, 2010), which have been described as key players in clathrin-mediated endocytosis. As such, members of the BAR domain containing protein family exemplify how scaffolding and wedging mechanism can be used independently from each other, but can also be applied simultaneously to efficiently induce and stabilize membrane curvature (Masuda and Mochizuki, 2010).

BAR domain containing proteins share the property of acting at sites of membrane dynamics, such as endocytic sites (Salazar et al., 2003; Soulet et al., 2005). Among the different endocytic routes that have been described in eukaryotic cells, clathrin-mediated endocytosis has been studied most extensively. This pathway requires a number of membrane-associated proteins to generate membrane curvature. The formation of clathrin-coated vesicles (CCVs) can be separated into four main steps (Kaksonen et al., 2005; Merrifield et al., 2005): 1) clathrin coat assembly and cargo selection to form the clathrin coated pit (CCP) 2) invagination of 


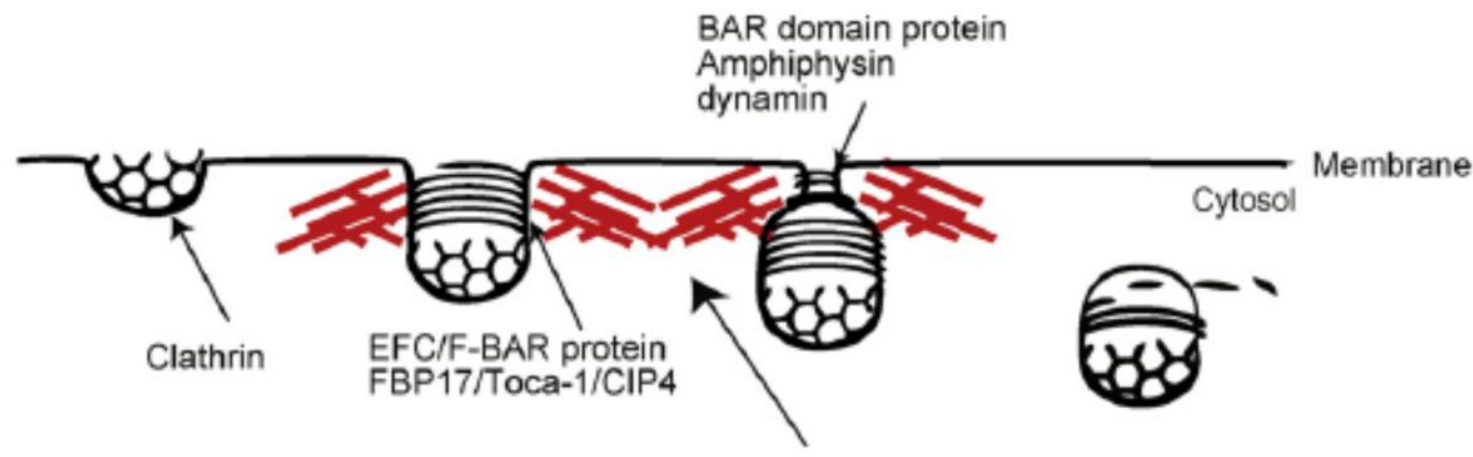

Actin polymerization

\author{
N-WASP-WP complex \\ Arp2/3 complex
}

Figure 10: Involvement of BAR domain proteins in clathrin-mediated endocytosis (adopted from (Suetsugu et al., 2010)).

Clathrin assembly forms the invaginations that may be recognized by the EFC/F-BAR domain. The binding of EFC/F-BAR domain proteins could further induce the invagination/tubulation of the plasma membrane, as well as the actin polymerization to constrict the tubules. The narrower tubules are eventually bound to the BAR domain. Actin polymerization and the dynamin recruited by the EFC/F$\mathrm{BAR}$ and BAR domain proteins induce the scission of the vesicles form the plasma membrane.

the CCP by recruitment of the actin polymerization machinery 3) neck formation at the CCP and 4) vesicle scission (Figure 10). For example FBP17 is a member of FBAR domain proteins, which is transiently recruited to CCPs in the late stage of clathrin-mediated endocytosis (Giuliani et al., 2009; Itoh et al., 2005; Suetsugu et al., 2010; Tsujita et al., 2006; Wu et al., 2010). Clathrin coat assembly probably leads to its accumulation at the foot of the hemispherical structure, which is consistent with its preference for low curvature membranes (Shimada et al., 2007). Subsequent FBP17 self-oligomerization then drives the CCP invagination by constricting the tube in order to form a neck. Additionally, activation of the actin nucleation machinery and further recruitment of dynamins may narrow the diameter of the neck for scission to take place. Here, classical BAR-domain proteins with a sharper curvature, such as amphiphysin (Takei et al., 1999) or endophilin (Farsad et al., 2001; Ferguson et al., 2009) are suggested to regulate the final constriction of the pits (Shimada et al., 2007). In this process of CME, multiple BAR domain proteins participate in multiple steps to generate and bind to different degrees of membrane curvature and recruit endocytic effectors. Also in yeast, CME represents a highly complex mechanism, involving around 60 different types of proteins of various functions (Weinberg and 
Drubin, 2012). Here, this process is locally restricted to defined sites of actin density at the plasma membrane, called actin patches. This demonstrates that also the yeast plasma membrane is able to segregate complex processes into distinct reaction compartments and is highly organized. 


\subsection{Plasma membrane organization in yeast Saccharomyces cerevisiae}

Although, plasma membrane domains are ubiquitously found and play a cruicial role in all different types of cell biological processes, the molecular mechanisms behind their formation are in most cases barely understood. In this regard, yeast S.cerevisiae provides an excellent model system to study this phenomenon, since its plasma membrane is highly organized into distinct domains and can be easily manipulated to allow visualization of proteins at their endogenous levels. As described in mammalian cells, also the yeast plasma membrane constitutes non-overlapping lateral domains (Grossmann et al., 2007; Malinska et al., 2003; Opekarova et al., 2005), which cover the vast majority of the plasma membrane. According to the presence of specific marker proteins, three main nonoverlapping compartments have been described so far: the membrane compartment containing 1) Can1 (MCC) and 2) Target of Rapamycin complex (TORC) 2 (MCT), which appear in a patchy pattern. The third, spreads as a network percolating in between the MCC and the MCT, the membrane compartment containing Pma1 (MCP) (Berchtold and Walther, 2009; Grossmann et al., 2007). Besides specific proteins, these domains also vary in their lipid composition. In particular, the MCC is suggested to be enriched in ergosterol, the major yeast sterol (Grossmann et al., 2007). In general, compartmentalization of the plasma membrane into domains of distinct protein and lipid content is believed to participate in the spatial control of cellular signaling (Fröhlich et al., 2009). Additionally, lateral organization is required for normal endocytosis of proteins located within these domains (Grossmann et al., 2008; Walther et al., 2006).

Compartmentalization of the yeast plasma membrane is achieved by large cytoplasmic protein complexes, termed eisosomes. These large, immobile structures are stably anchored underneath the plasma membrane at the cell cortex and their presence is required for proper plasma membrane organization of proteins and lipids. Eisosomes are composed primarily of two so far unknown proteins, Pil1 and Lsp1 (Figure 11) and have been described to mark static sites of endocytosis (Walther et al., 2006). Furthermore eisosomes colocalize with Sur7, a protein genetically interacting with endophilin, a BAR domain containing protein. Endophilin is a known endocytic effector, pointing to a connection between eisosomes and endocytosis. 


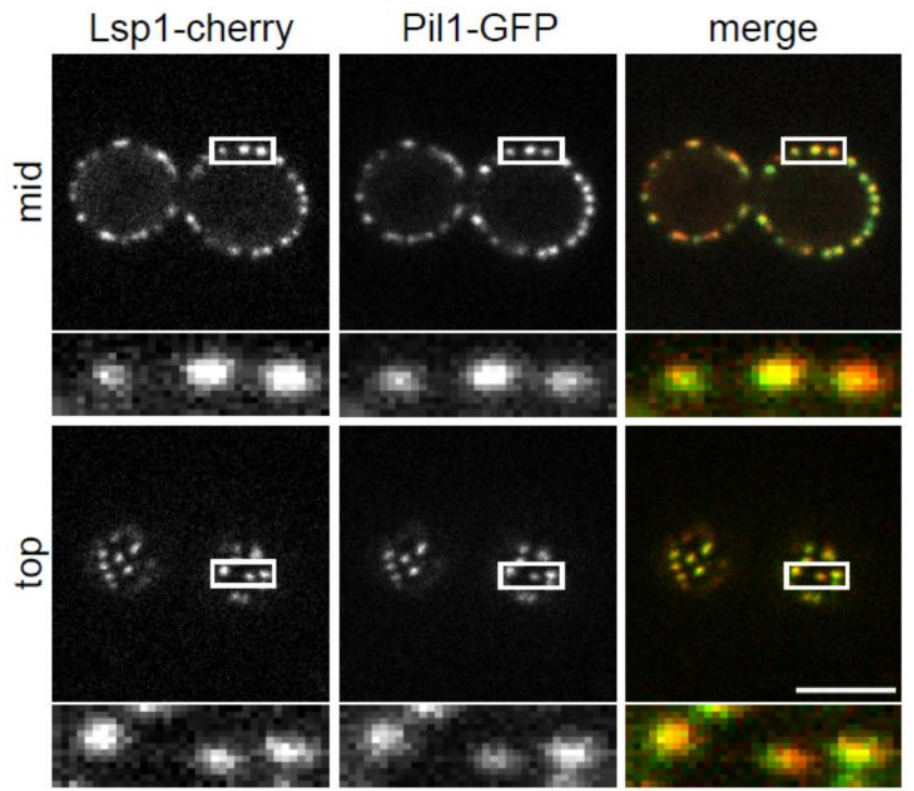

Figure 11: Eisosomes consist of Pil1 and Lsp1 and localize to the yeast cell cortex.

Fluorescence microscopy of cherrytagged Lsp1 and green fluorescent protein (GFP)-tagged Pil1 in yeast cells. Mid (upper panels) and top sections (lower panels) show both proteins co-localizing within eisosomes in a punctuate pattern at the plasma membrane. Insets show magnified views of the indicated areas (white boxes). Scale bar $5 \mu \mathrm{m}$.

This connection is further reflected by the genetic interactions of PIL1 and LSP1 with known endocytic effectors from yeast, such as RVS161, RVS167, PAN1, SLA1 and SLA2 (Michelot et al., 2010; Walther et al., 2006) as well as the physical interaction with the actin nucleation promoting factor Las17 (Michelot et al., 2010). Consistently, pulse-chase experiments investigating uptake of the fluorescent lipid dye FM-464 showed accumulation of this endocytosis marker into discrete foci after $20 \mathrm{~s}$ of application. Interestingly, all of these early endocytic intermediates colocalized with eisosomes, confirming the hypothesis that eisosomes mark sites of endocytosis (Walther et al., 2006). In turn though, not all eisosomes showed FM-464 accumulation, indicating that only a certain subset of eisosomes actively participates in endocytic events at a given time.

Eisosomes mediate the formation of MCC domains, which ultrastructurally appear as furrows in the plasma membrane (Stradalova et al., 2009). They are crucial for normal plasma membrane domain formation and endocytosis, since their absence, as for example in $\Delta$ pil1 cells, leads to altered distribution of lipids and proteins in the membrane. As observed by fluorescence microscopy, the plasma membrane domain pattern collapses in such cells, meaning all MCC proteins investigated so far mislocalize and are either evenly distributed in the membrane or accumulate into one or a few clusters, called eisosome remnants (Fröhlich et al., 2009; Grossmann et al., 2007; Walther et al., 2006). Furthermore, ergosterol loses its punctuate pattern with a more even localization in the plasma membrane and 


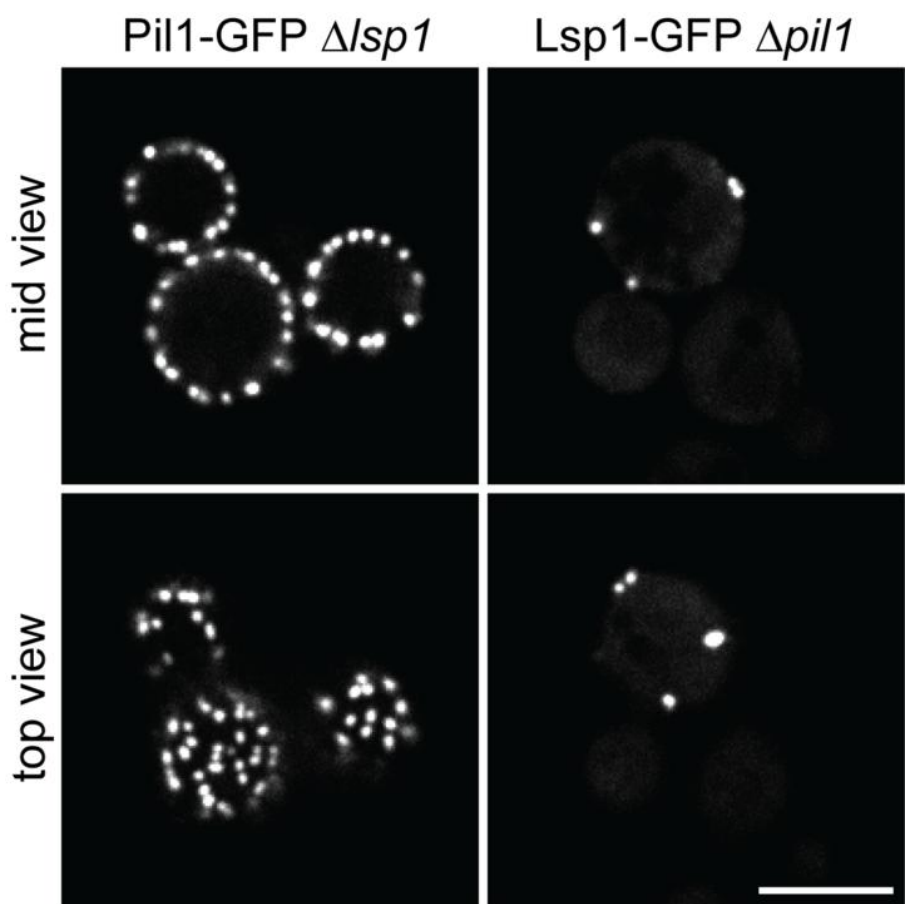

Figure 12: Pil1 is required for normal eisosome localization.

Fluorescence microscopy of GFPtagged Pil1 or Lsp1. In the absence of Lsp1, Pil1-GFP is sufficient to localize in the punctuate eisosome pattern (left panels). Lsp1-GFP alone cannot maintain the pattern and localizes to eisosome remnants (right panels).

accumulates at eisosome remnants (Grossmann et al., 2007). Ultrastructurally, these remnants appear by EM as large aberrant plasma membrane invaginations. Additionally, these cells have altered endocytosis (Grossmann et al., 2008; Walther et al., 2006) as well as cellular signalling (Zhang et al., 2004).

Eisosomes are not reminiscent of any other cellular assembly and stand out due to their characteristic uniform and punctuate pattern, with each eisosome being of relatively constant size. On average, a typical yeast cell has around 30 eisosomes, which are spread along the plasma membrane with a minimal distance from each other (Moreira et al., 2009). Their pattern arises during the cell cycle when the proteins are expressed and once established stays stable, meaning individual eisosomes do neither move nor exchange subunits (Walther et al., 2006). Strikingly, these huge protein clusters are mainly composed of two subunits only, Pil1 and Lsp1. With an estimated number of 115,000 copies per cell for Pil1 and 104,000 copies per cell for Lsp1, both proteins are highly abundant in yeast (de Godoy et al., 2008; Ghaemmaghami et al., 2003). From that, rough calculation estimates a total number of 3000 copies of each of the proteins per complex, since no significant cytoplasmic pool can be detected. These identical subunits, in turn must be repeatedly arranged within eisosomes, to yield a uniform and functional complex. 
Pil1 and Lsp1 are almost identical in their amino acid sequence. They share $72 \%$ of their residues, with the biggest variance in their C-termini, but based on their primary sequence neither of them contains any discernable functional domain,. Within eisosomes, each of these two proteins is represented with similar copy numbers. Consistently, purifications of either Pil1 or Lsp1 from yeast extracts coprecipitate equimolar amounts of the equivalent partner, respectively, indicating a stoichiometric complex (Walther et al., 2006). Although, Pil1 and Lsp1 almost resemble each other in their primary sequence information, they do not function redundantly in eisosome architecture. While the presence of Pil1 is crucial for eisosome assembly and plasma membrane organization in general, the equally abundant Lsp1 surprisingly cannot compensate for Pil1 loss (Figure 12, right panels). However, the molecular basis for this difference was not known so far. 


\section{Aim of the thesis}

In yeast, eisosomes help to organize the plasma membrane into lateral domains of distinct protein and lipid composition. However, the mechanistic details explaining how they bind to the membrane and function to organize lipids and proteins were completely unclear so far. Eisosomes show fascinating structural characteristics: i) they localize in a stable pattern along the whole plasma membrane ii) they are uniformly distributed, with each of them being at minimal distance from each other and iii) they have a constant size, indicating a relatively uniform number of subunits within each eisosome.

A major question about eisosome biology arises from these features, namely how are these structures molecularly built? To address this question, the first aim of my work was to biochemically characterize the structure and architecture of eisosomes at several levels of resolution. For an efficient analysis of eisosome structure, I established an in vitro system that allowed me to investigate these complexes on the basis of recombinant proteins. Therefore, I developped the expression of recombinant Pil1 and Lsp1 in E.coli and optimized their purification procedure, in order to yield protein of sufficient quantity and quality. Previous studies revealed that recombinant Pil1 self-assembles into long filamentous structures. Using a combination of different biochemical, biophysical and cell biological techniques, I characterized the self-assembly by eisosome proteins, Pil1 and Lsp1, into higher order structures. This included techniques such as sedimentation velocity gradients, fluorescence microscopy as well as negative stain and electron microscopy (EM), For a more detailed analysis at a higher level of resolution, I studied the structure of the filaments in their native state by cryo-electron tomography (cryo-ET), cryo-EM and three-dimensional (3D) image reconstruction. This part of my work was performed in collaboration with Prof. Dr. Kay Grünewald and Dr. Juha Huiskonen (Max-PlanckInstitute of Biochemistry, Germany and Division of Structural Biology, University of Oxford, UK).

Building up on the detailed structural analysis of eisosome proteins in vitro, my second aim was the characterization of membrane binding of recombinant Pil1 and Lsp1, using different approaches. First, I established spin-down experiments based on artificially generated lipid vesicles. Thereby, I studied lipid binding affinities, in 
order to unveil potential preferences for specific lipid species over others. In a second complementary approach, I visualized the proteins in the presence of liposomes to study the structure of such protein-liposome mixtures by cryo-ET as well as a combination of cryo-EM and 3D image reconstruction. Thereby, I generated a 3D model of the proteins in interaction with the membrane that revealed how the proteins align relatively to each other as well as to the membrane.

My third aim addressed an obvious paradox about the structure and architecture of eisosomes: in vitro eisosomes assemble into long helices, while in vivo they appear as regular dots at the plasma membrane by fluorescent microscopy. This raised the question how these structures correlate with each other. As an answer to this question is essential towards the understanding of eisosome function, I investigated their structure bound to the plasma membrane of yeast cells. Therefore, I collaborated with the laboratory of Prof. Dr. John Heuser (Washington University School of Medicine, Saint Louis, USA), in order to perform freeze-fracture and deepetch EM (DEEM). Using these techniques creates the unique opportunity to image true surfaces of biological membranes and allowed me to characterize the eisosome scaffold in yeast cells from two different perspectives: 1) the view from the outside of a cell onto the inner leaflet of the plasma membrane with eisosomes bound to it and 2) a direct view onto eisosomes, looking from the cytoplasm onto the plasma membrane. 


\section{Discussion}

Plasma membrane organization is a universal phenomenon of all different types of cells. Although, the lateral segregation of biological reactions constitutes an essential feature, the molecular mechanisms leading to such compartmentalization are mostly unknown. In yeast S.cerevisiae, organization of MCC domains is mediated by eisosomes. As such, they provide an inroad to answering this complex phenomenon. In this work, I have addressed the mechanistic details of plasma membrane domain organization by eisosomes using a combination of structural, biochemical, genetic and cell biological approaches to characterize their main components, Pil1 and Lsp1. My study revealed a fascinating, previously unrecognized self-assembling scaffold that directly binds to and organizes the yeast plasma membrane.

Eisosome proteins stand out due to their stable localization at the plasma membrane (Walther et al., 2006), as well as their presence in extremely high copy numbers (Pil1: 115.000 copies/cell; Lsp1: 104.000 copies/cell; (Hua et al., 2006). These characteristics support the idea of eisosomes playing a role in cellular architecture. As part of the cytoskelleton, tubulin and actin are prominent examples for such architectural proteins, which are highly abundant in cells (5.590 copies/cell for tubulin; 60.000 copies/per cell for actin (Ghaemmaghami et al., 2003; Norbeck and Blomberg, 1997)). However, in comparison to those, eisosome proteins are present with much higher copy numbers, so that Pil1 and Lsp1 could be classified as part of a previously unrecognized cytoskeleton, participating in plasma membrane organization. Consistent with this idea, my work unveils the mechanism of eisosomemediated plasma membrane domain organization, being based on self-assembly of its two main components Pil1 and Lsp1 into a protein scaffold that directly binds and locally deforms the membrane in a $\mathrm{PI}(4,5) \mathrm{P}_{2}$-specific manner.

\subsection{Pil1 and Lsp1 assemble into large multimeric complexes}

Although Pil1 and Lsp1 are almost identical in their amino acid sequence, they play a different architectural role in eisosome structure and assembly. However, the molecular detail behind this paradox was previously unknown. Simultaneously to my 
work in our laboratory, Dr. Natasza Ziołkowska solved the crystal structure of the stable core fragment of Lsp1 including residues 36 to 267 of the full-length protein (named "Lsp1ASIA") and identified the protein as member of the BAR domain containing protein superfamily. This protein family is evolutionary conserved and assembles into complexes on cellular membranes, thereby using the combined energy of multiple subunits to overcome the rigidity of the membrane in order to induce curvature. Other examples of BAR domains have been described to be soluble proteins that assemble into scaffolds upon membrane binding. In contrast, eisosome proteins Pil1 and Lsp1 self-assemble into higher order structures of

Figure 13: Model for the assembly of eisosomes on the plasma membrane.

Assembly of eisosomes can be separated conceptually into three steps: interactions of the proteins to form dimers (interaction 1), association of dimers to form thin filaments (interaction 2) and assembly into helices (interaction 3). Rings observed for Pil1 are interpreted in this model as side-products

of the filament to helix assembly.

crystal structure indentified the stable core domain of Lsp1 as a BAR domain containing protein. Members of this protein family are structurally characterized by anti-parallel association of two monomeric subunits into a banana-shaped dimer. I hypothesize that the same is true for Pil1, since both proteins resemble each other with more than $72 \%$ of their amino acid sequence. Second, the smallest asymmetric unit in each of my EM-derived structural models of either Pil1 or Lsp1 shows a twofold symmetry, strongly suggesting the presence of a dimeric repetitive element in the structures formed by the full-length proteins. These basic building blocks of BAR dimers then interact sidewise at their tips with each other to form a thin filament (interaction 2) (Figure 13). Subsequently, a third, lateral interaction would then lead to helix formation by curling up of thin filaments (interaction 3) (Figure 13). These are represented in the $3 \mathrm{D}$ reconstructions as ridges running on the surface of the structure, reflecting each turn of the helix. This characteristic striation pattern can also be observed in eisosomes investigated by DEEM of freeze-fractured whole yeast cells and plasma membrane fractions, respectively.

Out of these interfaces, interaction 3 is most likely quite flexible, since helices can be formed with significant variation in diameters and varying helical parameters. Furthermore, I predict, that interaction 3 is affected by post-translational modifications, such as phosphorylation. Pil1 and Lsp1 have been described as targets of Pkh-kinases in vivo (Fröhlich et al., 2009; Luo et al., 2008; Walther et al., 2007), 


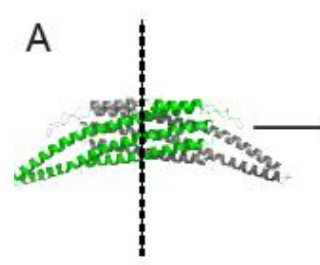

interaction 1

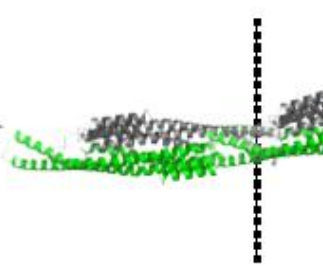

interaction 2

Figure 13: Model for the assembly of eisosomes on the plasma membrane.

Assembly of eisosomes can be separated conceptually into three steps: interactions of the proteins to form dimers (interaction 1), association of dimers to form thin filaments (interaction 2) and assembly into helices (interaction 3). Rings observed for Pil1 are interpreted in this model as side-products of the filament to helix assembly.

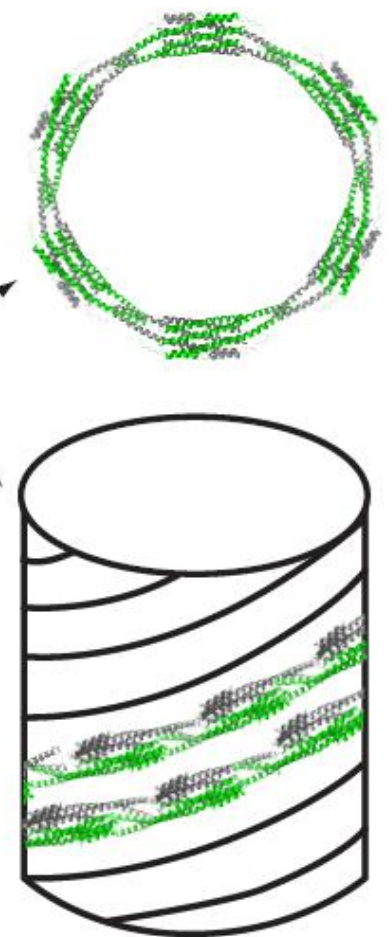

interaction 3

where either their overexpression, addition of the drug myriocin or other treatments increasing Pil1 phosphorylation lead to disassembly of eisosomes (Fröhlich et al., 2009; Luo et al., 2008; Walther et al., 2007). Consistent with these observations, interaction 3 is sensitive to phospho-mimicking mutations. In such mutants, replacement of phosphorylatable serine or threonine residues by a negatively charged aspartate mimicks the presence of a negatively charged phosphogroup in this position and thereby constant phosphorylation. In vivo, such a mutant of Pil1 ("pil1(4D)" with mutations S45D, S59D, S230D, T233D) shows disassembly of eisosomes, with most of the protein being either cytoplasmic or clustering into eisosome remnants at the plasma membrane. This effect can be reconstituted in vitro, since recombinant pil1(4D) also exhibits a disassembly phenotype, showing exclusive assembly into thin filaments and the absence of large helices. Two out of the four phosphorylation sites mutated in pil1(4D) are located in the N-terminus of the protein. Furthermore, N-terminal truncation of Pil1 (pil1 $\Delta N$ ) shows the same phenotype as pil1(4D) in vitro, further suggesting that this segment of the protein is required for interaction 3. Overall, Pil1 has at least 11 phosphorylation sites, so that additional phosphorylation on different residues other than in pil1(4D) may have other, more subtle effects on the structure, leading to local rearrangements, for 
example to adjust the local curvature of the lattice or the interaction with the membrane.

Although Pil1 and Lsp1 are almost identical in sequence, they differ in their significance for eisosome architecture. We propose that this difference is based on variance in the strength between these three interaction interfaces. In vitro, we observed different types of oligomeric structures for Pil1: rings, thin filaments and thick helices. In contrast, Lsp1 primarily formed thick helices highly similar to those of Pil1. The absence of thin filaments and rings in Lsp1 samples suggests a lower tendency to assemble into such structures. Hence, their filament forming interaction 2 might be weaker than that of Pil1, which shows prominent formation of filaments and ring structures. In contrast, Lsp1 helices appear longer and much more ordered in comparison to Pil1, which in turn argues for a stronger lateral interaction 3, mediating the formation of these structures. Moreover, we propose that Lsp1 helices are only stabilized by the collective energy of all end-to-end and lateral interactions. As such, smaller assemblies like rings and thin filaments are unstable and fall apart, which is consistent with the observation of barbed, unordered chains at the ends of Lsp1 helices and the much larger pool of free, non-assembled Lsp1 observed in sedimentation gradients as compared to Pil1. In this model, the formation of ring structures mostly seen for recombinant Pil1 would represent a side product, which forms when thin filaments close up on themselves, thereby preventing helix formation. Interestingly, among the few residues that differ between Lsp1 and Pil1, three are located directly on the tips of the banana-shaped dimer (E/K152, $H / Y 155$, $P / E 167)$. The presence of these amino acid differences being mostly accumulated in the tip regions of the dimer, supports the idea of variance in interaction strength between the different interfaces being the cause for the diverging behavior of Pil1 and Lsp1,.

In the EM-derived structural models of full-length eisosome proteins, the smallest asymmetric unit was invariably a dimer of either Pil1 or Lsp1. For Lsp1, this was confirmed by the crystal structure of its core domain, indentifying it as a BARdomain containing protein. Since both proteins are highly homologous and purify as a stoichiometric complex from yeast, it is possible that eisosomes assembled from Pil1 and Lsp1 form heterodimers in vivo, instead of homodimers assessed here. This idea is supported by homology modeling of a theoretical Pil1 structure based on the Lsp1 
ASIA core domain and subsequent generation of theoretical Pil1 homodimers or Pil1/Lsp1 heterodimers (NE Ziołkowska, unpublished observation). Subsequent assessment of energy profiles of such models indicated that Pil1/Lsp1 heterodimers would be energetically more stable structures than hypothetical Pil1 homodimers (NE Ziolkowska, unpublished observation). Interestingly, heterodimer formation has been described for other BAR domain containing proteins in yeast. Rvs161/Rvs167, the yeast homologues of endophilin/amphiphysin, are both members of the N-BAR protein family, tubulate liposomes in vitro and are implicated in endocytosis (Kaksonen et al., 2005; Youn et al., 2010). Interestingly, these proteins show heterodimer formation (Ren et al., 2006; Youn et al., 2010), but in the absence of either partner, each of the proteins can compensate the lack by forming homodimers, which can perform most of the functions of the heterodimer (Lombardi and Riezman, 2001; Navarro et al., 1997). The structural and functional analogy of these proteins, supports the hypothesis that this is true for Pil1 and Lsp1 as well, meaning that both proteins are capable of building homodimers, but form heterodimers in the presence of the other partner. Localization of Pil1 and Lsp1 by immunogold-labeling and EM on preparations allowing the view from the cytoplasm onto the plasma membrane showed that both proteins are present within eisosomes covering membrane furrows. However, in which specific way the proteins associate to form eisosomes and whether their different properties are used to modulate eisosome structure, is yet unclear.

\subsection{Pil1 and Lsp1 bind phosphoinositides}

Eisosomes are a fascinating case of cellular pattern formation. Besides their uniform size, eisosomes also maintain a minimal distance from each other. Outstanding questions are what restricts assembly of Pil1 in cells, preventing the formation of one giant eisosome rod, and how is their punctuate pattern achieved. When cells are manipulated to increase isotopically in size, new eisosomes form at maximal distance between existing ones and grow to the same dimension (Moreira et al., 2009). In order to yield such a system, eisosome size and localization have to be regulated. Earlier studies showed that eisosome size control is at least partially regulated by Pil1 expression levels and phosphorylation, with the latter responding to changes in sphingolipid synthesis (Fröhlich et al., 2009; Moreira et al., 2009). My 
work now shows that eisosome distribution is regulated by $\mathrm{PI}(4,5) \mathrm{P}_{2}$ levels. From my model, I predict that assembly into a stable helix with a membrane tubule inside (in vitro) or a furrow-like lattice (in vivo) results from direct interaction of dimers or thin filament pieces with the membrane (Figure 14). Furthermore, several lines of evidence support that Pil1 and Lsp1 interact directly with $\mathrm{PI}(4,5) \mathrm{P}_{2}$ : i) Pil1 and Lsp1 tubulate liposomes containing low amounts of $\mathrm{PI}(4,5) \mathrm{P}_{2}$; ii) nitrobenzoxadiazole (NBD)-labeled Pil1 yields a strong signal in the presence of $\mathrm{PI}(4,5) \mathrm{P}_{2}$-containing liposomes, representing membrane binding; iii) in sedimentation assays, Lsp1 interacts more strongly with $\mathrm{PI}(4,5) \mathrm{P}_{2}$ containing liposomes than with those containing other types of charged lipids at the same concentration; iv) inactivation of Mss4, leading to $\mathrm{PI}(4,5) \mathrm{P}_{2}$ depletion in the plasma membrane, has a strong effect on eisosome localization in vivo; v) Consistently, deletion of two $\mathrm{PI}(4,5) \mathrm{P}_{2}$ phosphatases (SJL1 and SJL2) and increased $\mathrm{PI}(4,5) \mathrm{P}_{2}$ levels lead to enlarged green fluorescent protein (GFP)-labelled Pil1 assemblies and vi) PIL1 and SJL1 show highly similar genetic interaction profiles in separately generated E-MAP datasets.

Direct $\mathrm{PI}(4,5) \mathrm{P}_{2}$ binding most likely mediates interaction of Pil1 and Lsp1 with the membrane and plays a critical role in eisosome function in vivo. Lowered $\mathrm{PI}(4,5) \mathrm{P}_{2}$ levels in the plasma membrane, as for example in mss4 mutant cells, lead to dissociation of the proteins from the membrane and aggregation into large eisosome remnants, most likely due to uncontrolled self-assembly. Additionally, our data suggest that $\mathrm{PI}(4,5) \mathrm{P}_{2}$ interaction is not only crucial for Pil1 and Lsp1 assembly,

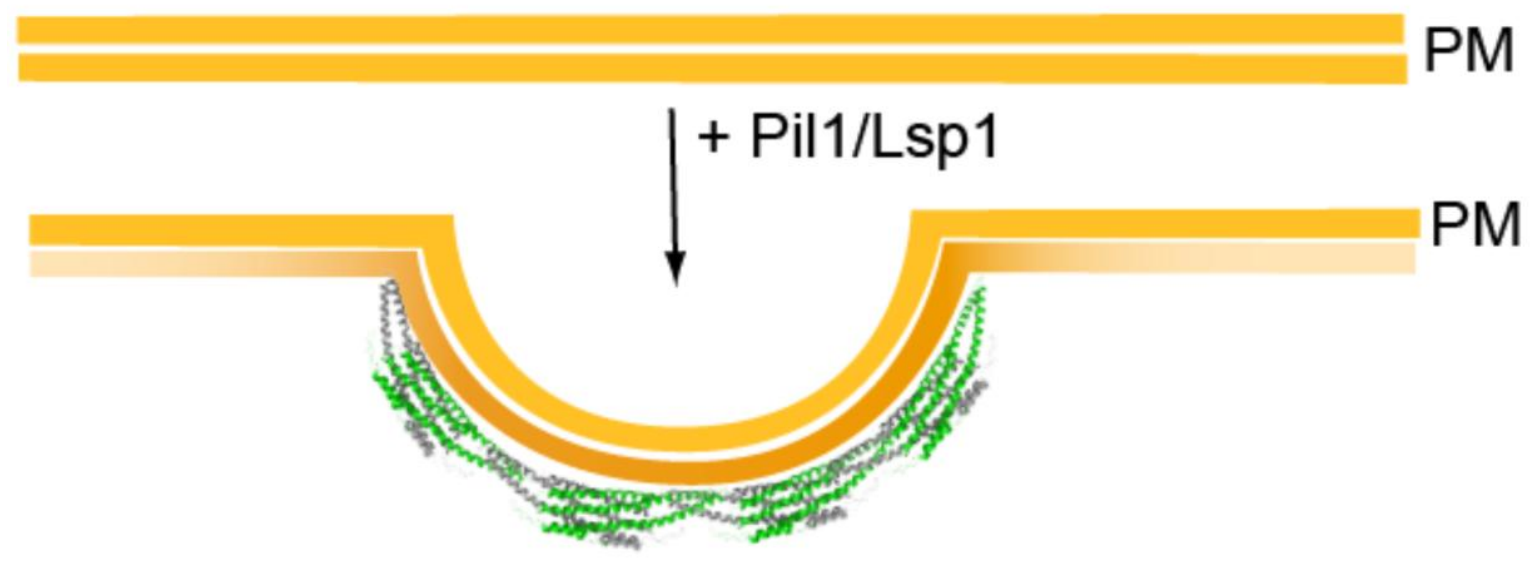

Figure 14: Model for the assembly of eisosomes on the plasma membrane.

On the plasma membrane, main eisosome components assemble into a scaffold similar to a halfhelix. See Discussion for details. 
but also for their function in regulating plasma membrane organization of the MCC domain. Specifically, the trans-membrane protein Sur7, loses its punctuate MCC localization and becomes uniformly distributed in the plasma membrane upon $\mathrm{PI}(4,5) \mathrm{P}_{2}$ depletion and consequent collapse of eisosomes in mss4 mutant cells. Moreover, $\mathrm{PI}(4,5) \mathrm{P}_{2}$ interaction and clustering may regulate eisosome distribution. In such a model, $\mathrm{PI}(4,5) \mathrm{P}_{2}$ binding of the many eisosome components would lead to accumulation of this lipid in the MCC and concomitantly its local depletion in the surrounding membrane region, by being the limiting factor. This could explain why new eisosomes never form right next to existing ones, but always at minimal distance. A prediction from this hypothesis is that $\mathrm{PI}(4,5) \mathrm{P}_{2}$ stabilizes Pil1 assemblies. Consistently, I observe such an effect in vitro, where liposome binding stabilized Pil1 to form very long helices compared to protein alone, thus validating this hypothesis. Additionally, this may also explain why eisosomes are not formed at bud-tips, where Pil1 and Lsp1 compete for lipid binding with many higher affinity $\mathrm{PI}(4,5) \mathrm{P}_{2}$-binding proteins that are targeted there. Of course this model does not exclude that other proteins and regulatory factors may act to fine-tune assembly and pattern formation. Good candidates for this are proteins encoded by genes whose mutations affect the eisosome pattern in cells, as for example Eis1/Ymr031c (Fröhlich et al., 2009) However, it is currently unknown whether these proteins play a direct structural role or function indirectly, for example by altering the phosphorylation state of eisosome proteins.

An important question to fully understand eisosome-mediated MCC domain organization is how the proteins interact with $\mathrm{PI}(4,5) \mathrm{P}_{2}$ molecularly. X-ray structure determination unveiled the stable core domain of Lsp1 as a BAR domain containing protein. Rigid body fitting of Lsp1 ASIA into EM-derived model of membrane-bound Lsp1, illustrated that the protein faces the membrane with its concave surface, as described for other members of this family (Frost et al., 2008). A striking feature of this region is a patch of positively charged amino acids that is conserved in fungi. I found that amino acids in this patch are required for efficient membrane binding in vitro, as well as for Pil1 function in plasma membrane organization in vivo. This positively charged surface patch likely interacts with the negatively charged headgroups of $\mathrm{PI}(4,5) \mathrm{P}_{2}$, since mutation of these amino acids leads to a loss of membrane binding affinity in vitro. Furthermore, mutations of residues in the patch lead to a phenotype of Pil1 localization in vivo, similar to the one observed after 
depleting $\mathrm{PI}(4,5) \mathrm{P}_{2}$ by inactivating Mss4, the kinase generating this lipid. Additionally, one of the phosphorylation sites mimicked in pil1(4D) (S59) is located within this patch, thereby introducing an opposite charge, which could explain at least in part the disassembly phenotype of pil1(4D) in vitro and in vivo.

In summary, perturbations in $\mathrm{PI}(4,5) \mathrm{P}_{2}$ binding in vivo, either by depletion of this lipid or mutations in the lipid interaction interface of Pil1 lead to its dissociation from the membrane, the formation of eisosome remnants as well as the loss of plasma membrane domain organization. As such, $\mathrm{PI}(4,5) \mathrm{P}_{2}$ interaction of eisosome proteins is crucial for eisosome assembly and function.

\subsection{Pil1 and Lsp1 sculpt membranes}

How can self-assembly of Pil1 and Lsp1 lead to the formation or stabilization of curved membranes, such as tubules and furrows? In general, two mechanisms how proteins generate and stabilize membrane curvature have been described, protein scaffolding of the membrane and insertion of an amphipathic alpha helix into the lipid-bilayer, a so-called "wedge" (Zimmerberg and Kozlov, 2006). However, both mechanisms are not mutually exclusive. Fitting of Lsp1 ASIA crystal structure into the EM-derived 3D maps of full-length Lsp1 with and without bound membranes showed that the protein apposes its concave surface towards the membrane, indicating a scaffolding mechanism. Strikingly, N-BAR domains share the highest structural similarity to the Lsp1 ASIA model. Members of this protein family are characterized by their combination of scaffolding and wedge mechanism and comprise an $\mathrm{N}$ terminal amphipatic segment that inserts into the lipid bilayer for efficient membrane bending (Gallop et al., 2006; Masuda and Mochizuki, 2010). In addition to a patch of positively charged amino acids, we identified the N-terminal segment of Pil1 and Lsp1 as required for efficient membrane binding. Although, this part of the protein is missing in our crystal structure, fitting in the EM-derived 3D maps shows that the proteins' N-termini are located within the concave surface of the BAR domain and point towards the membrane, theoretically allowing insertion into the lipid bilyer. Consistently, the reconstruction from full-length membrane-bound Lsp1 shows additional density pointing towards the surface of the membrane. This could represent the $\mathrm{N}$-terminal segment of the protein, which is unordered in the absence of membrane and thereby not detected in the 3D map of the proteins alone, but gains 
higher order upon membrane binding and thus, becomes detectable in the presence of the membrane. Although this additional density points towards the membrane surface, there is an obvious gap between the protein coat and the membrane, which is even larger in all other EM models. One explanation for this could be the cause of lipid disorder in the outer membrane leaflet due to the insertion of the N-terminus, thereby leading to the lack of resolved density in this region. Such phenomenon has been observed earlier for Endophilin, a member of the N-BAR domain protein family (Suresh and Edwardson, 2010). Hence, insertion of such an N-terminal segment of Pil1 and Lsp1 could contribute to membrane binding and bending and maybe also to membrane domain organization by the accumulation of a specific protein/lipid environment. However, whether the $\mathrm{N}$-termini of eisosome proteins insert an amphipathic alpha helix into the lipid bilayer according to the wedging mechanism or rather just externally associate with the membrane by electrostatic interactions with the lipid headgroups still needs to be confirmed.

Eisosome structure in general appears flexible. DEEM data of yeast plasma membrane fractions showed that the structures identified as eisosomes vary in their curvature. While some of them appear rather flat, other ones clearly show higher curvature (Figure 15A). This is consistent with the diameter variation observed in the reconstructions of Lsp1 alone, as well as membrane-bound Lsp1 and Pil1. All structures showed a repeating asymmetric unit with similar morphology, but the helical arrangement of these units varied with different diameters. This suggests that individual dimeric Pil1 and/or Lsp1 BAR domains might rotate relative to each other, leading to these different helical parameters (Figure 15B). This phenomenon has been described for other BAR domain containing proteins before, where CIP4 F-BAR domain dimers form tubules with altering diameter by being tilted relatively to the cylindrical axis (Frost et al., 2008). Such rearrangement could be supported by the flexibility of the proteins tip regions, as observed in the crystal structure of Lsp1 ASIA. In vivo, rotation of Pil1 and Lsp1 in eisosomes could result in the constriction or widening of the membrane furrow. Interestingly, pulse-chase experiments, monitoring the uptake of fluorescent lipid-dye FM4-64 shows accumulation into foci at some but not all eisosomes before its uptake. This suggests that a certain subset of eisosomes exhibits altered plasma membrane morphology in these sites in comparison to others. However, what leads to altered curvature and whether variation in eisosome 

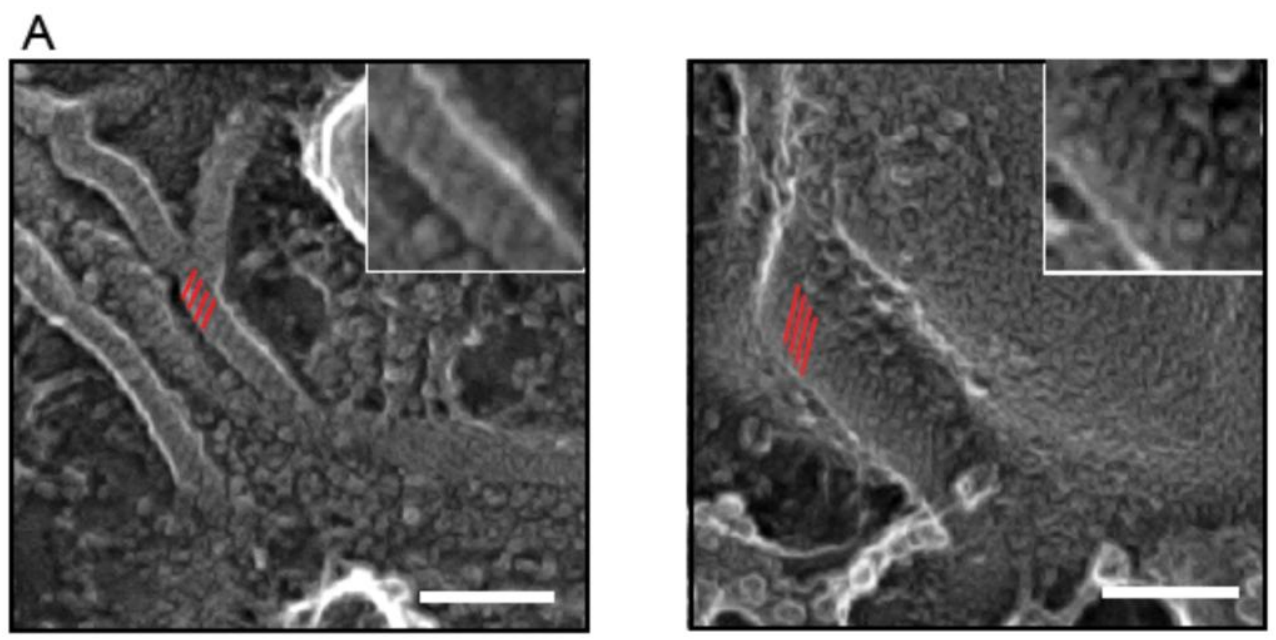

B
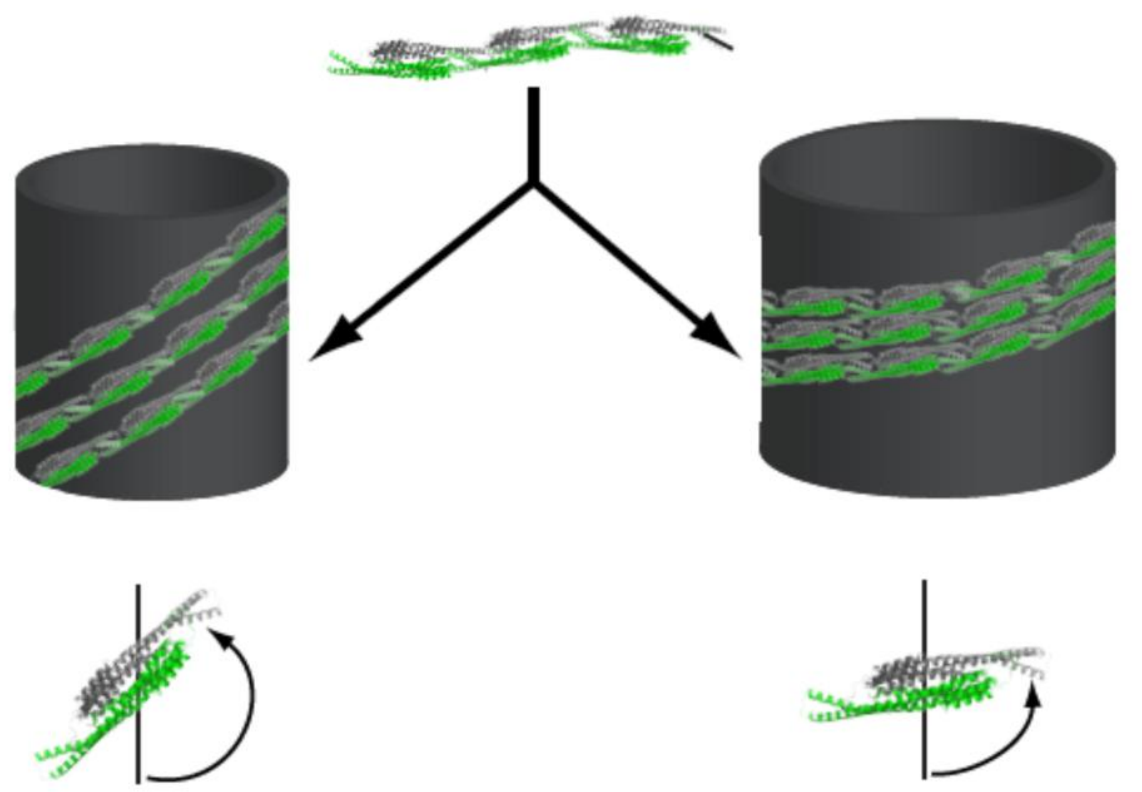

Figure 15: Eisosome proteins assemble into structures with varying diameter in vivo and in vitro.

(A) Representative image of the yeast plasma membrane from the cytosolic side. Eisosomes are represented by the striated structures (red parallel lines). Some eisosomes appear rather flat (right panel), while others show higher curvature (left panel). (B) Rotation of individual BAR dimers might lead to difference in curvature.

structure is linked to their function is completely unclear yet and needs further investigation.

Many of my considerations assume that the structural models generated for Pil1 and Lsp1 assemblies in vitro, reflect the structures of eisosomes in yeast. Several lines of evidence support this notion: First, we observed a very similar structure for recombinant Pil1 and Lsp1 assemblies as for eisosomes isolated from yeast cells. Second, phospho-mimicking mutations in Pil1 have a structural effect in 
vitro that is consistent with the phenotype of the mutations in yeast cells. Third and maybe most importantly, we detected eisosomes on the cytosolic face of the plasma membrane as elongated structures that show a striated pattern similar the helices formed by the recombinant proteins. Together, these data show that Pil1 and Lsp1 helices likely resemble eisosomes in vivo.

Although the structures are overall closely related to each other, there are also important differences. First, eisosomes purified from yeast cells contain both proteins, Pil1 and Lsp1 and thus, the building blocks of the lattice in vivo could be Pil1 and Lsp1 heterodimers, rather than the homodimers present in vitro. The different properties of the two proteins could be used individually to modulate the assembly. Second, filaments observed in vitro are closed cylinders that coat a membrane tubule, while in vivo eisosomes constitute a membrane furrow, likely resembling a half-cylinder. This is clearly demonstrated by 3D anaglyphes of DEEM, showing complimentary views on the plasma membrane from different perspectives (Figure 16): looking from the inside and from the outside of a yeast cell onto its plasma membrane, Although, in freeze-fracture DEEM images eisosome proteins are not directly visible, since they are still covered by a lipid monolayer, these two complementary views on eisosomes, show that eisosome proteins assemble at the plasma membrane into a lattice coating a furrow instead of a closed tubular structure.

From this obvious difference, questions arise about what restricts eisosome assembly into a furrow in vivo rather than a closed tube? Possibly, attachment of the membrane to the cell wall and the large turgor pressure could prevent the closure of the lattice to a helix similar to the ones seen in vitro. Another option includes the accumulation of a specified lipid or protein composition at the edges of the eisosome scaffold, which could restrict closure of the furrow. My in vitro study was restricted to the main eisosome components Pil1 and Lsp1 and did not take into account other 
A
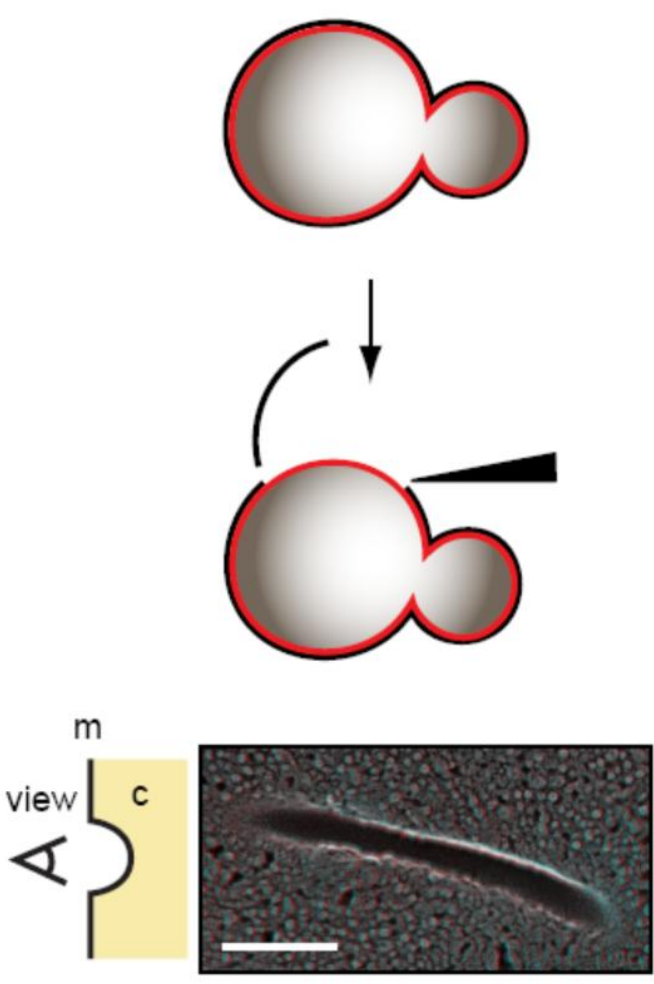

B
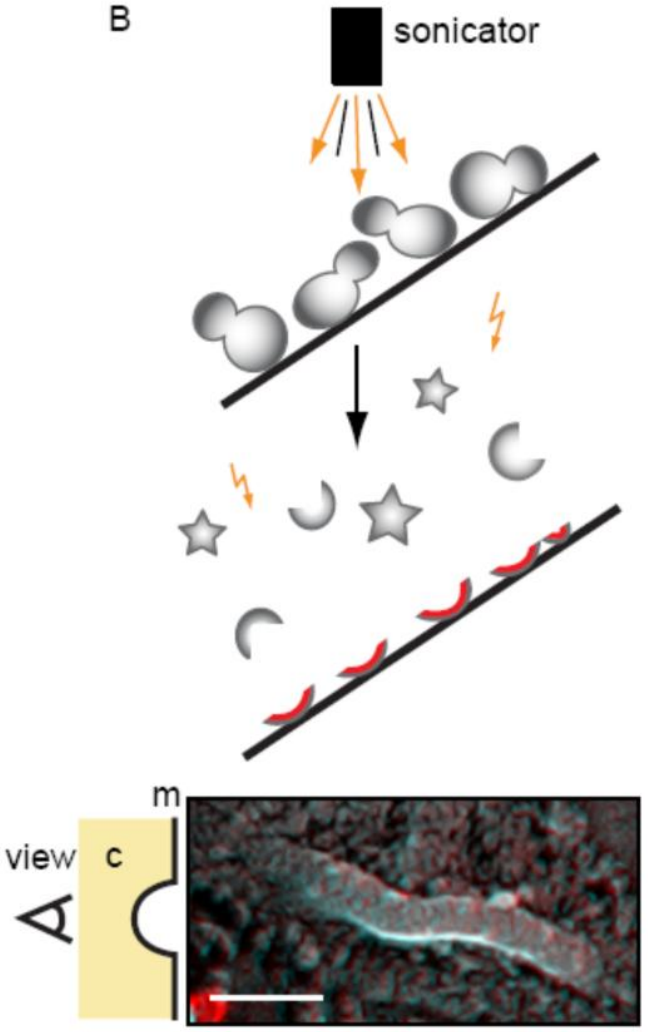

Figure 16: Eisosomes in vivo assemble into furrows instead of closed tubes

DEEM images showing views on the plasma membrane from different perspectives. (A) Schematic presentation of sample preparation workflow for freeze-fracture DEEM. Red areas represent the imaged surface. Lower left panel shows the view onto an eisosome generated by this procedure: looking from the outside of a cell onto the inner leaflet of the plasma membrane marked as $m$ ). (B) Schematic presentation of workflow for "unroofing" yeast cells, red areas in the cartoon represent imaged surfaces. Lower right panel shows the view onto an eisosome generated by this procedureand: looking from the cytoplasm (marked as c) onto the plasma membrane (marked as $\mathrm{m}$; red/cyan glasses are recommended for 3D view).

interaction partners and their potential impact on eisosome structure in vivo. Highthroughput screen using an algorithm that predicts stable protein complexes in S.cerevisiae identified Mrp8 as a new stable binding partner in a complex with eisosome proteins, Pil1 and Lsp1. Although this so far undescribed protein purifies as a trimeric complex of roughly stoichiometric similar amounts from yeast extracts, it does not play a role in eisosome architecture. Several lines of evidence suggest that Mrp8 does not have a direct structural impact on eisosomes i) Mrp8 does not colocalize with Pil1 in yeast cells ii) is a soluble cytoplasmic protein, and iii) is not required for normal eisosomes in vivo. Alternatively, Mrp8 could maybe function as a chaperone assisting eisosome formation by inhibiting self-assembly of proteins in the cytoplasm, which would explain its presence in a complex with eisosome proteins predicted in the screen as well as found in yeast extracts. However, the actual role of 
this abundant interaction partner of eisosome proteins still needs to be further analyzed, as well as potential other, so far unknown proteins that might have an impact on eisosome structure in vivo.

\subsection{Pil1 and Lsp1 stabilize plasma membrane domains}

Formation of the eisosome scaffold can explain how the lateral compartmentalization of the plasma membrane in domains of distinct lipid and protein composition is achieved mechanistically, in particular for the MCC. We propose that lipid binding and self-assembly of Pil1 and Lsp1 into a protein scaffold creates a specific membrane environment in the overlying MCC. This domain comprises local curvature and may be enriched in $\mathrm{PI}(4,5) \mathrm{P}_{2}$ based on the presence of the many binding sites for this lipid. This hypothesis is supported by two independently generated, unbiased, large scale E-MAP datasets that show highest correlation of genetic profiles between PIL1 and SJL1, a PI(4,5) $\mathrm{P}_{2}$ phosphatase. The absence of this function would have similar consequences as deletion of an enzyme that dephosphorylates this lipid -namely a rise in available $\mathrm{PI}(4,5) \mathrm{P}_{2}$. This finding suggests that eisosomes function in $\mathrm{PI}(4,5) \mathrm{P}_{2}$ turnover, such as the sequestration or hydrolysis of this lipid and could constitute a lipid store in the membrane. However, EMAP data are biased towards specific set of genes. These findings therefore do not rule out other functions of eisosomes when a different set is tested. Furthermore, specific $\mathrm{Pl}(4,5) \mathrm{P}_{2}$ binding of eisosomes could create a specialized membrane environment that selects for certain proteins and lipids, thereby excluding others. As such, eisosome scaffolds could for example cluster and stabilize nano-scale raft domains into larger micron-scale MCCs (Lingwood and Simons, 2010). Since the MCC is suggested to be enriched in ergosterol, eisosomes could utilize sterol/sphingolipid connectivity to form this domain (Grossmann et al., 2007). According to eisosomes being implicated in endocytosis, the accumulation of $\mathrm{PI}(4,5) \mathrm{P}_{2}$ in the MCC could also introduce a fission point in the membrane due to line-tension, similar to what has been suggested for clathrin-mediated endocytosis to induce vesicle scission (Liu et al., 2009). In this respect, it is interesting to note that we often observed membrane invaginations right next to eisosomes in my DEEM data of yeast plasma membrane fractions. 


\subsection{Evolutionary conservation of eisosomes}

One important question about eisosome biology refers to the evolutionary conservation of their molecular components. So far, the primary structure of eisosome proteins did not give any hints about homologous proteins in organisms other than fungi. This study reveals eisosome proteins Pil1 and Lsp1 as members of the BAR domain protein family, an ancient, evolutionary conserved group of proteins that binds and deforms membranes and has been implicated in endocytosis. Strikingly, the amino acid sequence of Pil1 and Lsp1 did not give any indication about the proteins' 3D conformation. Only the knowledge of their molecular structure allowed classification into this protein family. Specifically, the structure of Pil1 and Lsp1 is closely related to endocytic effectors, such as endophilin and amphiphysin BAR domains, with whom they also share several other intriguing similarities: both protein families consist of BAR-domains, can assemble into a membrane scaffold, are connected to $\mathrm{PI}(4,5) \mathrm{P}_{2}$ rich membranes and function with synaptojanin-proteins (Itoh et al., 2005). Furthermore, both sets of proteins were linked to endocytosis, but their deletions have mild defects on protein uptake in most systems (Brach et al., 2011; Grossmann et al., 2008; Schuske et al., 2003; Verstreken et al., 2003; Walther et al., 2006). In mammalian cells, endophilin recruits synaptojanin, the mammalian homologue of Sjl1, to endocytic sites through an SH3-domain (Schuske et al., 2003). Synaptojanin in turn acts preferentially on membranes that were curved for example by interaction with endophilin (Chang-lleto et al., 2011). Neither Pil1 nor Lsp1 contains such a SH3 domain. However, it was recently reported that membrane bending of endophilin is especially important for many of the protein's functions in Caenorhabditis elegans (Bai et al., 2010). Also Pil1 is capable of bending membranes and the high similarity of interaction profiles in pil1 $\Delta$ and sjl1 $\Delta$ further supports the idea that both genes participate in the same process, which reflects an important function of the Pil1 BAR domain. The yeast endophilin/amphiphysin homologues Rvs161/Rvs167 are also highly similar to Pil1 and Lsp1's architecture and molecular shape. These members of the N-BAR domain protein family are implicated in vesicle scission events of actin patch-dependent endocytosis (Liu et al., 2006; Liu et al., 2009). Interestingly, deletion of Lsp1 suppresses at least to some extend Rvs161 function (Walther et al., 2006), which could indicate partially overlapping tasks. 
Although, the mechanism of plasma membrane organization in mammalian cells is barely understood, some characterized examples show striking conceptual similarities to eisosome-driven plasma membrane organization. One example constitutes flask-shaped caveolae at the plasma membrane of mammalian cells. Besides their apparent difference in morphology, my data suggests analogous concepts to eisosomes in yeast: i) their major component caveolin 1 self-associates to form a protein coat at the plasma membrane, ii) caveolae form stable plasma membrane domains, iii) these domains are thought to be enriched in sphingolipids and sterols and iii) they are postulated to participate in cargo uptake only under specific conditions, but not in bulk endocytosis (Fernandez et al., 2002; Grossmann et al., 2008; Grossmann et al., 2007; Parton and Simons, 2007; Thomsen et al., 2002; Walther et al., 2006). Caveolae have also been described to function in lipid and membrane storage (Parton and Simons, 2007). Furthermore, recent studies showed that Caveolae also function as physiological membrane reservoir that accommodates to mechanical membrane stress. Based on the flask-shaped morphology of this membrane domain, they exhibit a larger membrane surface than flat membranes. Under conditions of osmotic swelling or stretching of the membrane Caveolae flatten, thereby counterbalancing membrane tension and disappear from the membrane (Sinha et al., 2011). However, whether eisosomes perform analogous function to Caveolae still needs to be further investigated.

In summary, formation of the eisosome protein scaffold can mechanistically explain how the yeast plasma membrane is organized in domains of distinct composition, in particular for the MCC: We posit that membrane binding and assembly by Pil1 and Lsp1 will create a specific environment in the overlaying MCC, which is locally curved and may have increased $\mathrm{PI}(4,5) \mathrm{P}_{2}$ concentration due to the presence of many binding sites for this lipid. This special environment then drives formation of the MCC domain. Most often, striking features are conserved between different biological systems. As such, the mechanistic feature of cells to organizing their plasma membranes into functional domains by self-assembly of a protein scaffold represents a universal cell biological principle. 


\section{References}

Babbey, C.M., N. Ahktar, E. Wang, C.C. Chen, B.D. Grant, and K.W. Dunn. 2006. Rab10 regulates membrane transport through early endosomes of polarized Madin-Darby canine kidney cells. Mol Biol Cell. 17:3156-3175.

Bagnat, M., S. Keranen, A. Shevchenko, and K. Simons. 2000. Lipid rafts function in biosynthetic delivery of proteins to the cell surface in yeast. Proc Natl Acad Sci U S A. 97:3254-3259.

Bai, J., Z. Hu, J.S. Dittman, E.C. Pym, and J.M. Kaplan. 2010. Endophilin functions as a membrane-bending molecule and is delivered to endocytic zones by exocytosis. Cell. 143:430-441.

Berchtold, D., and T.C. Walther. 2009. TORC2 plasma membrane localization is essential for cell viability and restricted to a distinct domain. Mol Biol Cell. 20:1565-1575.

Carmosino, M., G. Valenti, M. Caplan, and M. Svelto. 2010. Polarized traffic towards the cell surface: how to find the route. Biol Cell. 102:75-91.

Casal, E., L. Federici, W. Zhang, J. Fernandez-Recio, E.M. Priego, R.N. Miguel, J.B. DuHadaway, G.C. Prendergast, B.F. Luisi, and E.D. Laue. 2006. The crystal structure of the BAR domain from human Bin1/amphiphysin II and its implications for molecular recognition. Biochemistry. 45:12917-12928.

Daum, G., N.D. Lees, M. Bard, and R. Dickson. 1998. Biochemistry, cell biology and molecular biology of lipids of Saccharomyces cerevisiae. Yeast. 14:14711510.

Di Paolo, G., and P. De Camilli. 2006. Phosphoinositides in cell regulation and membrane dynamics. Nature. 443:651-657.

Dickson, R.C., C. Sumanasekera, and R.L. Lester. 2006. Functions and metabolism of sphingolipids in Saccharomyces cerevisiae. Prog Lipid Res. 45:447-465.

Drin, G., J.F. Casella, R. Gautier, T. Boehmer, T.U. Schwartz, and B. Antonny. 2007. A general amphipathic alpha-helical motif for sensing membrane curvature. Nat Struct Mol Biol. 14:138-146.

Farsad, K., N. Ringstad, K. Takei, S.R. Floyd, K. Rose, and P. De Camilli. 2001. Generation of high curvature membranes mediated by direct endophilin bilayer interactions. J Cell Biol. 155:193-200.

Ferguson, S.M., A. Raimondi, S. Paradise, H. Shen, K. Mesaki, A. Ferguson, O. Destaing, G. Ko, J. Takasaki, O. Cremona, O.T. E, and P. De Camilli. 2009. Coordinated actions of actin and BAR proteins upstream of dynamin at endocytic clathrin-coated pits. Dev Cell. 17:811-822.

Ford, M.G., I.G. Mills, B.J. Peter, Y. Vallis, G.J. Praefcke, P.R. Evans, and H.T. McMahon. 2002. Curvature of clathrin-coated pits driven by epsin. Nature. 419:361-366.

Fra, A.M., E. Williamson, K. Simons, and R.G. Parton. 1995. De novo formation of caveolae in lymphocytes by expression of VIP21-caveolin. Proc Natl Acad Sci U S A. 92:8655-8659.

Fröhlich, F., K. Moreira, P.S. Aguilar, N.C. Hubner, M. Mann, P. Walter, and T.C. Walther. 2009. A genome-wide screen for genes affecting eisosomes reveals Nce102 function in sphingolipid signaling. J Cell Biol. 185:1227-1242.

Frost, A., R. Perera, A. Roux, K. Spasov, O. Destaing, E.H. Egelman, P. De Camilli, and V.M. Unger. 2008. Structural basis of membrane invagination by F-BAR domains. Cell. 132:807-817. 
Gallop, J.L., C.C. Jao, H.M. Kent, P.J. Butler, P.R. Evans, R. Langen, and H.T. McMahon. 2006. Mechanism of endophilin N-BAR domain-mediated membrane curvature. EMBO J. 25:2898-2910.

Gallop, J.L., and H.T. McMahon. 2005. BAR domains and membrane curvature: bringing your curves to the BAR. Biochem Soc Symp:223-231.

Giuliani, C., F. Troglio, Z. Bai, F.B. Patel, A. Zucconi, M.G. Malabarba, A. Disanza, T.B. Stradal, G. Cassata, S. Confalonieri, J.D. Hardin, M.C. Soto, B.D. Grant, and G. Scita. 2009. Requirements for F-BAR proteins TOCA-1 and TOCA-2 in actin dynamics and membrane trafficking during Caenorhabditis elegans oocyte growth and embryonic epidermal morphogenesis. PLoS Genet. 5:e1000675.

Grossmann, G., J. Malinsky, W. Stahlschmidt, M. Loibl, I. Weig-Meckl, W.B. Frommer, M. Opekarova, and W. Tanner. 2008. Plasma membrane microdomains regulate turnover of transport proteins in yeast. $J$ Cell Biol. 183:1075-1088.

Grossmann, G., M. Opekarova, J. Malinsky, I. Weig-Meckl, and W. Tanner. 2007. Membrane potential governs lateral segregation of plasma membrane proteins and lipids in yeast. EMBO J.26:1-8.

Guerriero, C.J., Y. Lai, and O.A. Weisz. 2008. Differential sorting and Golgi export requirements for raft-associated and raft-independent apical proteins along the biosynthetic pathway. J Biol Chem. 283:18040-18047.

Hancock, J.F. 2006. Lipid rafts: contentious only from simplistic standpoints. Nat Rev Mol Cell Biol. 7:456-462.

Harder, T., and K. Simons. 1997. Caveolae, DIGs, and the dynamics of sphingolipidcholesterol microdomains. Curr Opin Cell Biol. 9:534-542.

Harlan, J.E., P.J. Hajduk, H.S. Yoon, and S.W. Fesik. 1994. Pleckstrin homology domains bind to phosphatidylinositol-4,5-bisphosphate. Nature. 371:168-170.

Haslam, R.J., H.B. Koide, and B.A. Hemmings. 1993. Pleckstrin domain homology. Nature. 363:309-310.

Henne, W.M., H.M. Kent, M.G. Ford, B.G. Hegde, O. Daumke, P.J. Butler, R. Mittal, R. Langen, P.R. Evans, and H.T. McMahon. 2007. Structure and analysis of FCHo2 F-BAR domain: a dimerizing and membrane recruitment module that effects membrane curvature. Structure. 15:839-852.

Hoekstra, D., D. Tyteca, and I.S.C. van. 2004. The subapical compartment: a traffic center in membrane polarity development. J Cell Sci. 117:2183-2192.

Honerkamp-Smith, A.R., P. Cicuta, M.D. Collins, S.L. Veatch, M. den Nijs, M. Schick, and S.L. Keller. 2008. Line tensions, correlation lengths, and critical exponents in lipid membranes near critical points. Biophys J. 95:236-246.

Honerkamp-Smith, A.R., S.L. Veatch, and S.L. Keller. 2009. An introduction to critical points for biophysicists; observations of compositional heterogeneity in lipid membranes. Biochim Biophys Acta. 1788:53-63.

Hu, J., Y. Shibata, C. Voss, T. Shemesh, Z. Li, M. Coughlin, M.M. Kozlov, T.A. Rapoport, and W.A. Prinz. 2008. Membrane proteins of the endoplasmic reticulum induce high-curvature tubules. Science. 319:1247-1250.

Hurley, J.H. 2006. Membrane binding domains. Biochim Biophys Acta. 1761:805811.

Ikonen, E. 2008. Cellular cholesterol trafficking and compartmentalization. Nat Rev Mol Cell Biol. 9:125-138.

Ipsen, J.H., G. Karlstrom, O.G. Mouritsen, H. Wennerstrom, and M.J. Zuckermann. 1987. Phase equilibria in the phosphatidylcholine-cholesterol system. Biochim Biophys Acta. 905:162-172. 
Itoh, T., K.S. Erdmann, A. Roux, B. Habermann, H. Werner, and P. De Camilli. 2005. Dynamin and the actin cytoskeleton cooperatively regulate plasma membrane invagination by BAR and F-BAR proteins. Dev Cell. 9:791-804.

Jacob, R., M. Heine, M. Alfalah, and H.Y. Naim. 2003. Distinct cytoskeletal tracks direct individual vesicle populations to the apical membrane of epithelial cells. Curr Biol. 13:607-612.

Janmey, P.A., and P.K. Kinnunen. 2006. Biophysical properties of lipids and dynamic membranes. Trends Cell Biol. 16:538-546.

Kahya, N., D. Scherfeld, K. Bacia, B. Poolman, and P. Schwille. 2003. Probing lipid mobility of raft-exhibiting model membranes by fluorescence correlation spectroscopy. J Biol Chem. 278:28109-28115.

Kaksonen, M., C.P. Toret, and D.G. Drubin. 2005. A modular design for the clathrinand actin-mediated endocytosis machinery. Cell. 123:305-320.

Koster, G., M. VanDuijn, B. Hofs, and M. Dogterom. 2003. Membrane tube formation from giant vesicles by dynamic association of motor proteins. Proc Natl Acad Sci U S A. 100:15583-15588.

Kusumi, A., Y. Sako, and M. Yamamoto. 1993. Confined lateral diffusion of membrane receptors as studied by single particle tracking (nanovid microscopy). Effects of calcium-induced differentiation in cultured epithelial cells. Biophys J. 65:2021-2040.

Kutateladze, T.G. 2010. Translation of the phosphoinositide code by PI effectors. Nat Chem Biol. 6:507-513.

Lajoie, P., J.G. Goetz, J.W. Dennis, and I.R. Nabi. 2009. Lattices, rafts, and scaffolds: domain regulation of receptor signaling at the plasma membrane. $J$ Cell Biol. 185:381-385.

Lapierre, L.A., R. Kumar, C.M. Hales, J. Navarre, S.G. Bhartur, J.O. Burnette, D.W. Provance, Jr., J.A. Mercer, M. Bahler, and J.R. Goldenring. 2001. Myosin vb is associated with plasma membrane recycling systems. Mol Biol Cell. 12:18431857.

Lee, A.G. 2003. Lipid-protein interactions in biological membranes: a structural perspective. Biochim Biophys Acta. 1612:1-40.

Lee, E., M. Marcucci, L. Daniell, M. Pypaert, O.A. Weisz, G.C. Ochoa, K. Farsad, M.R. Wenk, and P. De Camilli. 2002. Amphiphysin 2 (Bin1) and T-tubule biogenesis in muscle. Science. 297:1193-1196.

Lee, M.C., L. Orci, S. Hamamoto, E. Futai, M. Ravazzola, and R. Schekman. 2005. Sar1p N-terminal helix initiates membrane curvature and completes the fission of a COPII vesicle. Cell. 122:605-617.

Lee, S.H., F. Kerff, D. Chereau, F. Ferron, A. Klug, and R. Dominguez. 2007. Structural basis for the actin-binding function of missing-in-metastasis. Structure. 15:145-155.

Lemmon, M.A. 2008. Membrane recognition by phospholipid-binding domains. Nat Rev Mol Cell Biol. 9:99-111.

Lentz, B.R., D.A. Barrow, and M. Hoechli. 1980. Cholesterol-phosphatidylcholine interactions in multilamellar vesicles. Biochemistry. 19:1943-1954.

Levental, I., M. Grzybek, and K. Simons. 2010. Greasing their way: lipid modifications determine protein association with membrane rafts. Biochemistry. 49:63056316.

Lingwood, D., J. Ries, P. Schwille, and K. Simons. 2008. Plasma membranes are poised for activation of raft phase coalescence at physiological temperature. Proc Natl Acad Sci U S A. 105:10005-10010. 
Lingwood, D., and K. Simons. 2010. Lipid rafts as a membrane-organizing principle. Science. 327:46-50.

Lisanti, M.P., P.E. Scherer, J. Vidugiriene, Z. Tang, A. Hermanowski-Vosatka, Y.H. Tu, R.F. Cook, and M. Sargiacomo. 1994. Characterization of caveolin-rich membrane domains isolated from an endothelial-rich source: implications for human disease. J Cell Biol. 126:111-126.

Malinska, K., J. Malinsky, M. Opekarova, and W. Tanner. 2003. Visualization of protein compartmentation within the plasma membrane of living yeast cells. Mol Biol Cell. 14:4427-4436.

Masuda, M., and N. Mochizuki. 2010. Structural characteristics of BAR domain superfamily to sculpt the membrane. Semin Cell Dev Biol. 21:391-398.

Matlin, K.S., and K. Simons. 1984. Sorting of an apical plasma membrane glycoprotein occurs before it reaches the cell surface in cultured epithelial cells. J Cell Biol. 99:2131-2139.

Matter, K. 2000. Epithelial polarity: sorting out the sorters. Curr Biol. 10:R39-42.

Mattila, P.K., A. Pykalainen, J. Saarikangas, V.O. Paavilainen, H. Vihinen, E. Jokitalo, and P. Lappalainen. 2007. Missing-in-metastasis and IRSp53 deform $\mathrm{PI}(4,5) \mathrm{P} 2$-rich membranes by an inverse BAR domain-like mechanism. J Cell Biol. 176:953-964.

Mayer, B.J., R. Ren, K.L. Clark, and D. Baltimore. 1993. A putative modular domain present in diverse signaling proteins. Cell. 73:629-630.

McMahon, H.T., and J.L. Gallop. 2005. Membrane curvature and mechanisms of dynamic cell membrane remodelling. Nature. 438:590-596.

Mellman, I. 1996. Endocytosis and molecular sorting. Annu Rev Cell Dev Biol. 12:575-625.

Merrifield, C.J., D. Perrais, and D. Zenisek. 2005. Coupling between clathrin-coatedpit invagination, cortactin recruitment, and membrane scission observed in live cells. Cell. 121:593-606.

Michelot, A., M. Costanzo, A. Sarkeshik, C. Boone, J.R. Yates, 3rd, and D.G. Drubin. 2010. Reconstitution and protein composition analysis of endocytic actin patches. Curr Biol. 20:1890-1899.

Moreira, K.E., T.C. Walther, P.S. Aguilar, and P. Walter. 2009. Pil1 controls eisosome biogenesis. Mol Biol Cell. 20:809-818.

Munro, S. 2003. Lipid rafts: elusive or illusive? Cell. 115:377-388.

Murata, M., J. Peranen, R. Schreiner, F. Wieland, T.V. Kurzchalia, and K. Simons. 1995. VIP21/caveolin is a cholesterol-binding protein. Proc Natl Acad Sci U S A. 92:10339-10343.

Nelson, W.J., and C. Yeaman. 2001. Protein trafficking in the exocytic pathway of polarized epithelial cells. Trends Cell Biol. 11:483-486.

Opekarova, M., K. Malinska, L. Novakova, and W. Tanner. 2005. Differential effect of phosphatidylethanolamine depletion on raft proteins: further evidence for diversity of rafts in Saccharomyces cerevisiae. Biochim Biophys Acta. 1711:87-95.

Paladino, S., D. Sarnataro, R. Pillich, S. Tivodar, L. Nitsch, and C. Zurzolo. 2004. Protein oligomerization modulates raft partitioning and apical sorting of GPIanchored proteins. J Cell Biol. 167:699-709.

Parton, R.G., and K. Simons. 2007. The multiple faces of caveolae. Nat Rev Mol Cell Biol. 8:185-194.

Pelkmans, L., and M. Zerial. 2005. Kinase-regulated quantal assemblies and kissand-run recycling of caveolae. Nature. 436:128-133. 
Peter, B.J., H.M. Kent, I.G. Mills, Y. Vallis, P.J. Butler, P.R. Evans, and H.T. McMahon. 2004. BAR domains as sensors of membrane curvature: the amphiphysin BAR structure. Science. 303:495-499.

Pfeiffer, S., S.D. Fuller, and K. Simons. 1985. Intracellular sorting and basolateral appearance of the $\mathrm{G}$ protein of vesicular stomatitis virus in Madin-Darby canine kidney cells. J Cell Biol. 101:470-476.

Qualmann, B., D. Koch, and M.M. Kessels. 2011. Let's go bananas: revisiting the endocytic BAR code. EMBO J. 30:3501-3515.

Rajendran, L., and K. Simons. 2005. Lipid rafts and membrane dynamics. J Cell Sci. 118:1099-1102.

Roland, J.T., A.K. Kenworthy, J. Peranen, S. Caplan, and J.R. Goldenring. 2007. Myosin Vb interacts with Rab8a on a tubular network containing EHD1 and EHD3. Mol Biol Cell. 18:2828-2837.

Roth, M.G. 2004. Phosphoinositides in constitutive membrane traffic. Physiol Rev. 84:699-730.

Rothberg, K.G., J.E. Heuser, W.C. Donzell, Y.S. Ying, J.R. Glenney, and R.G. Anderson. 1992. Caveolin, a protein component of caveolae membrane coats. Cell. 68:673-682.

Roux, A., G. Cappello, J. Cartaud, J. Prost, B. Goud, and P. Bassereau. 2002. A minimal system allowing tubulation with molecular motors pulling on giant liposomes. Proc Natl Acad Sci U S A. 99:5394-5399.

Salazar, M.A., A.V. Kwiatkowski, L. Pellegrini, G. Cestra, M.H. Butler, K.L. Rossman, D.M. Serna, J. Sondek, F.B. Gertler, and P. De Camilli. 2003. Tuba, a novel protein containing bin/amphiphysin/Rvs and Dbl homology domains, links dynamin to regulation of the actin cytoskeleton. J Biol Chem. 278:4903149043.

Sargiacomo, M., P.E. Scherer, Z. Tang, E. Kubler, K.S. Song, M.C. Sanders, and M.P. Lisanti. 1995. Oligomeric structure of caveolin: implications for caveolae membrane organization. Proc Natl Acad Sci U S A. 92:9407-9411.

Sargiacomo, M., M. Sudol, Z. Tang, and M.P. Lisanti. 1993. Signal transducing molecules and glycosyl-phosphatidylinositol-linked proteins form a caveolinrich insoluble complex in MDCK cells. J Cell Biol. 122:789-807.

Schuck, S., M.J. Gerl, A. Ang, A. Manninen, P. Keller, I. Mellman, and K. Simons. 2007. Rab10 is involved in basolateral transport in polarized Madin-Darby canine kidney cells. Traffic. 8:47-60.

Schwencke, C., R.C. Braun-Dullaeus, C. Wunderlich, and R.H. Strasser. 2006. Caveolae and caveolin in transmembrane signaling: Implications for human disease. Cardiovasc Res. 70:42-49.

Seet, L.F., and W. Hong. 2006. The Phox (PX) domain proteins and membrane traffic. Biochim Biophys Acta. 1761:878-896.

Sheetz, M.P., and S.J. Singer. 1974. Biological membranes as bilayer couples. A molecular mechanism of drug-erythrocyte interactions. Proc Natl Acad Sci U S A. 71:4457-4461.

Shenoy-Scaria, A.M., D.J. Dietzen, J. Kwong, D.C. Link, and D.M. Lublin. 1994. Cysteine3 of Src family protein tyrosine kinase determines palmitoylation and localization in caveolae. J Cell Biol. 126:353-363.

Shibata, Y., J. Hu, M.M. Kozlov, and T.A. Rapoport. 2009. Mechanisms shaping the membranes of cellular organelles. Annu Rev Cell Dev Biol. 25:329-354.

Shimada, A., H. Niwa, K. Tsujita, S. Suetsugu, K. Nitta, K. Hanawa-Suetsugu, R. Akasaka, Y. Nishino, M. Toyama, L. Chen, Z.J. Liu, B.C. Wang, M. Yamamoto, T. Terada, A. Miyazawa, A. Tanaka, S. Sugano, M. Shirouzu, K. 
Nagayama, T. Takenawa, and S. Yokoyama. 2007. Curved EFC/F-BARdomain dimers are joined end to end into a filament for membrane invagination in endocytosis. Cell. 129:761-772.

Simons, K., and E. Ikonen. 1997. Functional rafts in cell membranes. Nature. 387:569-572.

Simons, K., and D. Toomre. 2000. Lipid rafts and signal transduction. Nat Rev Mol Cell Biol. 1:31-39.

Simons, K., and G. van Meer. 1988. Lipid sorting in epithelial cells. Biochemistry. 27:6197-6202.

Simons, K., and W.L. Vaz. 2004. Model systems, lipid rafts, and cell membranes. Annu Rev Biophys Biomol Struct. 33:269-295.

Singer, S.J., and G.L. Nicolson. 1972. The fluid mosaic model of the structure of cell membranes. Science. 175:720-731.

Smart, E.J., Y. Ying, W.C. Donzell, and R.G. Anderson. 1996. A role for caveolin in transport of cholesterol from endoplasmic reticulum to plasma membrane. $J$ Biol Chem. 271:29427-29435.

Soulet, F., D. Yarar, M. Leonard, and S.L. Schmid. 2005. SNX9 regulates dynamin assembly and is required for efficient clathrin-mediated endocytosis. Mol Biol Cell. 16:2058-2067.

Sprong, H., P. van der Sluijs, and G. van Meer. 2001. How proteins move lipids and lipids move proteins. Nat Rev Mol Cell Biol. 2:504-513.

Suetsugu, S., K. Toyooka, and Y. Senju. 2010. Subcellular membrane curvature mediated by the BAR domain superfamily proteins. Semin Cell Dev Biol. 21:340-349.

Suresh, S., and J.M. Edwardson. 2010. The endophilin N-BAR domain perturbs the structure of lipid bilayers. Biochemistry. 49:5766-5771.

Takei, K., V.I. Slepnev, V. Haucke, and P. De Camilli. 1999. Functional partnership between amphiphysin and dynamin in clathrin-mediated endocytosis. Nat Cell Biol. 1:33-39.

Tarricone, C., B. Xiao, N. Justin, P.A. Walker, K. Rittinger, S.J. Gamblin, and S.J. Smerdon. 2001. The structural basis of Arfaptin-mediated cross-talk between Rac and Arf signalling pathways. Nature. 411:215-219.

Toenjes, K.A., M.M. Sawyer, and D.I. Johnson. 1999. The guanine-nucleotideexchange factor Cdc24p is targeted to the nucleus and polarized growth sites. Curr Biol. 9:1183-1186.

Tsujita, K., S. Suetsugu, N. Sasaki, M. Furutani, T. Oikawa, and T. Takenawa. 2006. Coordination between the actin cytoskeleton and membrane deformation by a novel membrane tubulation domain of $\mathrm{PCH}$ proteins is involved in endocytosis. J Cell Biol. 172:269-279.

van der Rest, M.E., A.H. Kamminga, A. Nakano, Y. Anraku, B. Poolman, and W.N. Konings. 1995. The plasma membrane of Saccharomyces cerevisiae: structure, function, and biogenesis. Microbiol Rev. 59:304-322.

van Meer, G. 1989. Lipid traffic in animal cells. Annu Rev Cell Biol. 5:247-275.

van Meer, G., E.H. Stelzer, R.W. Wijnaendts-van-Resandt, and K. Simons. 1987. Sorting of sphingolipids in epithelial (Madin-Darby canine kidney) cells. J Cell Biol. 105:1623-1635.

van Meer, G., D.R. Voelker, and G.W. Feigenson. 2008. Membrane lipids: where they are and how they behave. Nat Rev Mol Cell Biol. 9:112-124.

Veatch, S.L., P. Cicuta, P. Sengupta, A. Honerkamp-Smith, D. Holowka, and B. Baird. 2008. Critical fluctuations in plasma membrane vesicles. ACS Chem Biol. 3:287-293. 
Walther, T.C., J.H. Brickner, P.S. Aguilar, S. Bernales, C. Pantoja, and P. Walter. 2006. Eisosomes mark static sites of endocytosis. Nature. 439:998-1003.

Wang, Q., M.V. Navarro, G. Peng, E. Molinelli, S.L. Goh, B.L. Judson, K.R. Rajashankar, and H. Sondermann. 2009. Molecular mechanism of membrane constriction and tubulation mediated by the F-BAR protein Pacsin/Syndapin. Proc Natl Acad Sci U S A. 106:12700-12705.

Waterman-Storer, C.M., and E.D. Salmon. 1998. Endoplasmic reticulum membrane tubules are distributed by microtubules in living cells using three distinct mechanisms. Curr Biol. 8:798-806.

Weinberg, J., and D.G. Drubin. 2012. Clathrin-mediated endocytosis in budding yeast. Trends Cell Biol. 22:1-13.

Weisz, O.A., and E. Rodriguez-Boulan. 2009. Apical trafficking in epithelial cells: signals, clusters and motors. J Cell Sci. 122:4253-4266.

Wu, M., B. Huang, M. Graham, A. Raimondi, J.E. Heuser, X. Zhuang, and P. De Camilli. 2010. Coupling between clathrin-dependent endocytic budding and FBAR-dependent tubulation in a cell-free system. Nat Cell Biol. 12:902-908.

Xu, Y., H. Hortsman, L. Seet, S.H. Wong, and W. Hong. 2001. SNX3 regulates endosomal function through its PX-domain-mediated interaction with Ptdlns(3)P. Nat Cell Biol. 3:658-666.

Yu, J.W., and M.A. Lemmon. 2001. All phox homology (PX) domains from Saccharomyces cerevisiae specifically recognize phosphatidylinositol 3phosphate. J Biol Chem. 276:44179-44184.

Zhu, G., J. Chen, J. Liu, J.S. Brunzelle, B. Huang, N. Wakeham, S. Terzyan, X. Li, Z. Rao, G. Li, and X.C. Zhang. 2007. Structure of the APPL1 BAR-PH domain and characterization of its interaction with Rab5. EMBO J. 26:3484-3493.

Zimmerberg, J., and M.M. Kozlov. 2006. How proteins produce cellular membrane curvature. Nat Rev Mol Cell Biol. 7:9-19. 


\section{Acknowledgments}

I greatly acknowledge Tobias Walther for beeing a great supervisor, for his contagious enthusiasm and many motivating and inspiring discussions about science as well as life in general. He inspired me and taught me to always think about the big picture. Furthermore, I want to thank Kay Grünewald for all the time an effort to teach me so much about electron microscopy.

I am also very grateful to Prof. Dr. Stefan Jentsch for supervising this thesis. I would also like to thank the members of my committee Dr. Charles David, Dr. Heinrich Jung and Dr. Angelika Böttger.

Thanks to the entire Walther lab, Florian Fröhlich, Doris Berchtold, Natalie Krahmer, Florian Wilfling, Romain Christiano, Natasza Ziolkowska and Michael Rehmann for creating an atmosphere, which was a pleasure to work in, for many helpful chats about scientific as well as personal matters and so many great moments we spent together.

Furthermore, I would like to thank Juha Huiskonen for his unlimited patience in teaching me electron microscopy, his helpfulness in each phase of this project and great times in the bunker. Furthermore, I greatly thank Dori, Ira, Janina, Barbara, Stefan, Christoph, Caro und Milena for being amazing friends and their way of making me laugh.

Zuletzt und ganz besonders möchte ich meiner Familie, Marita, Volker, Mine und Julia danken! Für die vielen großen und kleinen Momente der Unterstützung, der Geduld und Motivation, die mir geholfen haben auch schwierige Situationen zu meistern. Tommy danke ich für sehr viel, aber vor allem für den Beweis, dass zuletzt doch alles gut wird. Ganz besonderer Dank gilt auch Elke, ohne sie wäre diese Arbeit nicht möglich gewesen! 


\section{Curriculum vitae}

\section{Lena Karotki}

Date of birth: 23. May 1982

Nationality: German

Home address:

Andechser Strasse 1

81369 München

cell phone: $+49179-1373442$

e-mail: karotki@biochem.mpg.de

\section{Business address:}

Max-Planck-Institute of Biochemistry

Organelle Dynamics and Architecture

Am Klopferspitz 18

82152 Martinsried

Phone: +49 (89) 8578-3443

Fax: $\quad+49(89) 8578-2412$

\section{PUBLICATIONS}

Karotki L, Huiskonen JT, Krogan NJ, Heuser J, Grünewald K, Walther TC. Self-assembly of eisosome core components drives plasma membrane organization; (in preparation; Cell)

Ziolkovska N.E, Karotki L, Rehmann M, Walther T.C. Membrane organization by BAR-domain proteins. (in preparation)

Wang H, Kakaradov B, Collins SR, Karotki L, Fiedler D, Shales M, Shokat KM, Walther TC, Krogan NJ, Koller D. A Complex-Based Reconstruction of the S. cerevisiae Interactome; Mol Cell Proteomics. 2009 Jan 27

\section{EDUCATION}

07/2007 - present Max-Planck-Institute of Biochemistry, Martinsried, Germany PhD thesis

10/2003 - 04/2007 University of Tübingen, Germany

Diploma in Biology

Major: cell biology / immunology

Minors: virology, physiology

10/2001 - 08/2003 University of Hohenheim, Germany

Prediploma in Biology

\section{RESEARCH EXPERIENCE}

07/2007 - present Max-Planck-Institute of Biochemistry, Martinsried, Germany

$\mathrm{PhD}$ thesis supervised by Dr. Tobias Walther, Organelle Dynamics and Architecture

Title: "Structure and Architecture of Eisosomes"

07/2006 to 03/2007 Massachusetts Institute of Technology, Cambridge, USA

Diploma thesis supervised by Dr. Thomas Schwartz, Department of Biology Title: "Proline isomerization-dependant homodimerization of the B-subunit of the eukaryotic SRP receptor" 
07/2004 - 07/2006 Max-Planck-Institute for Developmental Biology, Tübingen, Germany

Student assistant in the laboratory of Dr. Robert Geisler

006/2005 - 06/2005 Friedrich-Miescher-Laboratory, Tübingen, Germany Internship in the laboratory of Dr. Anne Spang

05/2005 - 06/20005 Max-Planck-Institute for Developmental Biology, Tübingen, Germany Internship in the laboratory of Dr. Hermann Aberle

\section{AWARDS}

$03 / 2008-06 / 2010$

PhD fellowship Boehringer Ingelheim Fonds (B.I.F.)

$08 / 2006-12 / 2006$

Fellowship from the German Academic Exchange Service (DAAD)

\section{SKILLS/VOLUNTEER WORK}

Languages

German (native language), English (fluent), Italian (basic), French (basic)

$10 / 2007-10 / 2009$

Departmental Spokesperson, Max-Planck-Institute of Biochemistry 


\section{Declaration of Individual Contributions}

Publication 1: Wang H, Kakaradov B, Collins SR, Karotki L, Fiedler D, Shales M, Shokat KM, Walther TC, Krogan NJ, and Koller D. (2009). A complex-based reconstruction of the Saccharomyces cerevisiae interactome. Mol Cell Proteomics 8 , 1361-1381

Lena Karotki contributed Figure 4a. She designed and performed the experiment shown in this Figure.

Publication 2: Ziolkowska NE, Karotki L, Rehman M, Huiskonen JT, and Walther TC (2011). Eisosome-driven plasma membrane organization is mediated by BAR domains. Nat Struct Mol Biol 18, 854-856.

Lena Karotki established the protocols for protein expression and purification. She contributed to the design of the protein construct used for crystallisation. She contributed to the design and performance corresponding to the membrane binding characterization. She co-wrote parts of the paper.

Publication 3: Karotki L, Huiskonen JT, Stefan JS, Ziolkowska NE, Roth R, Surma MA, Krogan NJ, Emr SD, Heuser J, Grünewald K, Walther TC (2011). Eisosome Proteins Assemble into a Membrane Scaffold. J Cell Biol 195, 889-902.

Lena Karotki designed and performed all experiments except the computational analysis for the 3D reconstructions and rigid body fitting, the EMAP and the NBDfluorescence spectroscopy experiments. She created all figures, except the ones corresponding to the EMAP and the NBD-fluorescence spectroscopy experiments. She co-wrote all parts of the paper.

Prof. Dr. Stefan Jentsch 


\section{Reprints of the Publications}




\title{
A Complex-based Reconstruction of the Saccharomyces cerevisiae Interactome ${ }^{\star_{[}}$
}

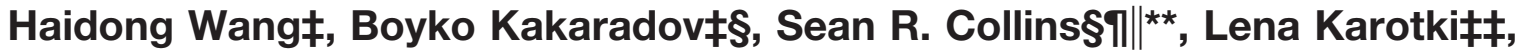

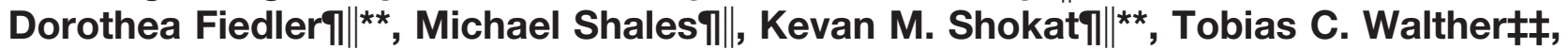

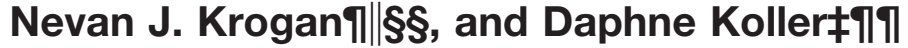

\begin{abstract}
Most cellular processes are performed by proteomic units that interact with each other. These units are often stoichiometrically stable complexes comprised of several proteins. To obtain a faithful view of the protein interactome we must view it in terms of these basic units (complexes and proteins) and the interactions between them. This study makes two contributions toward this goal. First, it provides a new algorithm for reconstruction of stable complexes from a variety of heterogeneous biological assays; our approach combines state-of-the-art machine learning methods with a novel hierarchical clustering algorithm that allows clusters to overlap. We demonstrate that our approach constructs over $40 \%$ more known complexes than other recent methods and that the complexes it produces are more biologically coherent even compared with the reference set. We provide experimental support for some of our novel predictions, identifying both a new complex involved in nutrient starvation and a new component of the eisosome complex. Second, we provide a high accuracy algorithm for the novel problem of predicting transient interactions involving complexes. We show that our complex level network, which we call ComplexNet, provides novel insights regarding the protein-protein interaction network. In particular, we reinterpret the finding that "hubs" in the network are enriched for being essential, showing instead that essential proteins tend to be clustered together in essential complexes and that these essential complexes tend to be large. Molecular \& Cellular Proteomics 8:1361-1381, 2009.
\end{abstract}

Biological processes exhibit a hierarchical structure in which the basic working units, proteins, physically associate to form stoichiometrically stable complexes. Complexes in-

From the $¥$ Computer Science Department, Stanford University, Stanford, California 94305, ๆDepartment of Cellular and Molecular Pharmacology, University of California, San Francisco, California 94158, ||The California Institute for Quantitative Biomedical Research and ${ }^{* *}$ Howard Hughes Medical Institute, San Francisco, California 94158-2330, and $\ddagger \ddagger$ Max Planck Institute for Biochemistry, Am Klopferspitz 18, 82152 Martinsried, Germany

${ }^{\circ}$ Author's Choice-Final version full access.

Received, October 27, 2008, and in revised form, January 26, 2009

Published, MCP Papers in Press, January 27, 2009, DOI 10.1074/ mcp.M800490-MCP200 teract with individual proteins or other complexes to form functional modules and pathways that carry out most cellular processes. Such higher level interactions are more transient than those within complexes and are highly dependent on temporal and spatial context. The function of each protein or complex depends on its interaction partners. Therefore, a faithful reconstruction of the entire set of complexes in the cell is essential to identifying the function of individual proteins and complexes as well as serving as a building block for understanding the higher level organization of the cell, such as the interactions of complexes and proteins within cellular pathways. Here we describe a novel method for reconstruction of complexes from a variety of biological assays and a method for predicting the network of interactions relating these core cellular units (complexes and proteins).

Our reconstruction effort focuses on the yeast Saccharomyces cerevisiae. Yeast serves as the prototypical case study for the reconstruction of protein-protein interaction networks. Moreover the yeast complexes often have conserved orthologs in other organisms, including human, and are of interest in their own right. Several studies (1-4) using a variety of assays have generated high throughput data that directly measure protein-protein interactions. Most notably, two high quality data sets $(3,4)$ used tandem affinity purification (TAP) $)^{1}$ followed by MS to provide a proteome-wide measurement of protein complexes. These data provide the basis for attempting a comprehensive reconstruction of a large fraction of the protein complexes in this organism. Indeed a number of works $(5,6)$ have attempted such a reconstruction. Generally speaking, all use the same general procedure: one or more data sources are used to estimate a set of affinities between pairs of proteins, essentially measuring the likelihood of that pair to participate together in a complex. These affinities

${ }^{1}$ The abbreviations used are: TAP, tandem affinity purification; EMAP, epistatic miniarray profile; EM, expectation maximization; GO, Gene Ontology; HAC, hierarchical agglomerative clustering; HACO, hierarchical agglomerative clustering with overlap; JC, Jaccard coefficient; PCC, Pearson correlation coefficient; PE, purification enrichment; TF, transcription factor; Y2H, yeast two-hybrid; MIPS, Munich Information Center for Protein Sequences; MCL, Markov clustering; SGD, Saccharomyces Genome Database; NSC, nutrient starvation complex; DIP, Database of Interacting Proteins; SJC, scaled Jaccard coefficient; ROC, receiver operating characteristic; $\mathrm{CCl}$, complexcomplex interaction; NB, naïve Bayes. 
induce a weighted graph whose nodes are proteins and whose edges encode the affinities. A clustering algorithm is then used to construct complexes, sets of proteins that have high affinity in the graph. Although similar at a high level, the different methods differ significantly on the design choices made for the key steps in the process.

Recent works (since 2006) all focus on processing the proteome-wide TAP-MS data and using the results to define complexes. Gavin et al. (3), Collins et al. (7), and Hart et al. (5) all use probabilistic models that compare the number of interactions observed between proteins in the data versus the number expected in some null model. Collins et al. (7) and Hart et al. (5) both used all three of the available high throughput data sets (2-4) in an attempt to provide a unified interaction network. The two unified networks resulting from these studies were shown to have large overlap and to achieve comparable agreement with the set of co-complex interactions in the MIPS data set (8) that are collated from previous small scale studies. The interaction graphs resulting from the computed affinity scores are then clustered to produce a set of identified complexes. Gavin et al. (3), Hart et al. (5), and Pu et al. (6) all use a Markov clustering (MCL) (9) procedure; Collins et al. (7) use a hierarchical agglomerative clustering (HAC) procedure but do not suggest a computational procedure for using the resulting dendrogram to produce specific complex predictions.

Despite the fairly high quality of these networks and the agreement between them, they still contain many false positives and negatives. False negatives can arise, for example, from the difficulty in detecting interactions involving low abundance proteins or membrane proteins or from cases where the tag added to the bait protein during TAP-MS prevents binding of the bait to its interacting partners. False positives can arise, for example, from complexes that share components or from the contaminants that bind to the bait nonspecifically after cell lysis. Therefore, the set of complexes derived from the protein-protein interaction network alone has limited accuracy. Less than $20 \%$ of the MIPS complexes (8), which are derived from reliable small scale experiments, are exactly captured by the predictions of Pu et al. (6) or by those of Hart et al. (5).

In this study, we constructed a method that generates a set of complexes with higher sensitivity and coverage by integrating multiple sources of data, including mRNA gene expression data, cellular localization, and yeast two-hybrid data. The data integration approach was used in some early works on predicting protein-protein interactions $(10,11)$ and more recently by Qiu and Noble (12), but these studies focus only on predicting pairs of proteins in the same complex and not on reconstructing entire complexes. Many recent studies (13-21) have successfully integrated multiple types of data to predict functional linkage between proteins, constructing a graph whose pairwise affinity score summarizes the information from different sources of data. However, because the data integration is not trained toward predicting complexes, the high affinity pairs contain transient binding partners and even protein pairs that never interact directly but merely function in the same pathways. When these graphs are clustered, the clusters correspond to a variety of cellular entities, including pathways, functional modules, or co-expression clusters. We developed a data integration approach that is aimed directly at the problem of predicting stoichiometrically stable complexes.

We used a two-phase automated procedure that we trained on a new high quality reference set that we generated from annotations in MIPS and SGD and from manual curation of the literature. In the first phase, we used boosting (22), a state-of-the-art machine learning method, to train an affinity function that is specifically aimed at predicting whether two proteins are co-complexed. Unlike most other learning methods, boosting is capable of inducing useful features by combining different aspects of the raw data, making it particularly well suited to a data integration setting. Once we generated the learned affinity graph over pairs of proteins, we predicted complexes by using a novel clustering algorithm called hierarchical agglomerative clustering with overlap (HACO). The HACO algorithm is a simple and elegant extension of HAC that addresses many of its limitations, such as the irreversible commitment to a possibly incorrect clustering decision. HACO can be applied to any setting where HAC is applied; given the enormous usefulness of HAC for the analysis of biological data sets of many different types (e.g. Refs. 7, 23, and 24), we believe that HACO may be applicable in a broad range of other tasks.

To validate our approach, we tested the ability of our methods and other methods to predict reference complexes that were not used in training. By integrating multiple sources of data, we recovered more reference complexes than other state-of-the-art methods $(5,6)$ when applied to the same set of yeast proteins. We also validated our predicted set of complexes against external data sources that are not used in the training. In all cases, our predictions were shown to be more coherent than other methods and, in many cases, more coherent even than the set of reference complexes.

A detailed examination of our predicted complexes suggests that many of them were previously known but not included in our (comprehensive) reference set, suggesting that our complexes form a valuable new set of reference complexes. In several cases, our predicted complexes were not previously characterized. We experimentally validated two of these predictions: a new component in the recently characterized eisosome complex (25), which marks the site of endocytosis in eukaryotes, and a newly characterized sixprotein complex, including four phosphatases, that appears to be involved in the response to nutrient starvation and that we named the nutrient starvation complex (NSC).

The complex-based view provides a new perspective on the analysis and reconstruction of the protein interaction net- 
work. In the past, Jeong et al. (26) have suggested that the degree of a protein in an interaction network is positively correlated with its essentiality and have argued that "hubs" in the network are more likely to be essential because they are involved in more interactions. Our analysis presents a complex-based alternative view: essential proteins tend to cluster together in essential complexes (5), and essential complexes tend to be large; thus, the essential hubs in the network are often members in large complexes comprised mostly of essential proteins. We also reformulate the task of reconstructing the protein interaction network. Rather than considering interactions between individual proteins (27-29), a somewhat confusing network that confounds interactions within complexes and interactions between complexes, we tackle the novel task of predicting a comprehensive protein interaction network that involves both individual proteins and larger complexes. We argue that these entities are the right building blocks in reconstructing cellular processes, providing a view of cellular interaction networks that is both easier to interpret than the complex network of interactions between individual proteins and more faithful to biological reality. Moreover a complex, which is a stable collection of many proteins that act together, provides a more robust basis for predicting interactions as we can combine signals for all its constituent proteins, reducing sensitivity to noise.

To accomplish this goal, we constructed a reference set of complex-complex interactions, considering two complexes to interact if they are significantly enriched for reliable interactions between their components. We further augmented this set with a hand-curated list of established complex-complex interactions. We then used a machine learning approach to detect the "signature" of such interactions from a large set of assays that are likely to be indicative. We explored different machine learning methods and showed that a partially supervised naïve Bayes model, where we learned the model from both labeled and unlabeled interactions, provides the best performance. This model was applied both to our predicted complexes and to individual proteins, providing a new, comprehensive reconstruction of the $S$. cerevisiae interaction network, which can be downloaded from our project Web page. ${ }^{2}$ We showed that entities that are predicted to interact are more likely to share the same functional categories. A detailed investigation of our new predicted interactions presents many that are established in the literature as well as some that are novel but consistent, presenting plausible hypotheses for further investigation.

\section{EXPERIMENTAL PROCEDURES Complex Prediction}

Constructing a Set of Reference Complexes-We compiled a reference set of complexes by combining literature-derived results from small scale experiments in MIPS (8) and SGD (31) with a hand-curated

\footnotetext{
${ }^{2}$ Complex-complex interactions (CCl) (dags.stanford.edu/CCl/).
}

list (see our supporting Web site ${ }^{3}$ ) that we generated. The MIPS, SGD, and hand-curated sets contain 225, 195, and 164 complexes, respectively (supplemental Fig. S1a). Below we describe our method for establishing correspondence between the three lists and combining them into a high confidence reference set suitable for training our method and for evaluating the accuracy of its predictions.

Our approach consisted of five processing steps. First, we merged similar complexes from the original lists (see below), resulting in a list of 543 complexes. Second, we removed 112 redundant complexes that were proper subsets of other complexes. Third, we removed the five largest complexes: the four ribosomal subunits and the small nucleolar ribonucleoprotein complex; these complexes are so large that they greatly overwhelm the signal both in training the method and in evaluating the results. Fourth, we restricted the complexes to the set of 2195 proteins that have adequate amount of experimental evidence (see below). Finally we removed single protein complexes, arriving at the final list of 340 complexes. With at least two and on average 4.9 proteins per complex, this set of complexes contained 1100 unique proteins and a total of 1661 protein members, showing that the reference complexes contain notable overlap (proteins that are shared by multiple complexes).

In the first step of this merging process, we define each candidate complex from the three curated lists as a node in an undirected graph (or network). Two complexes are connected by an edge if they overlap significantly, i.e. their Jaccard similarity coefficient is greater than 0.7 (see Jaccard coefficient (JC) metric below) with an edge weight equal to the JC value. We found 422 isolated nodes in the graph, corresponding to unique complexes that do not overlap significantly with any other complexes in the list. The task of merging similar complexes is equivalent to that of finding several types of connected components in this graph. A complete subgraph with average edge weight of 1 is equivalent to a group of complexes with identical protein content that appear under multiple names in at least two of the curated lists. We found 66 such groups, which correspond to complexes that we regard as very high confidence because of multiple corroborating evidence. A complete subgraph in the rest of the network with average edge weight less than 1 (but greater than 0.7 ) is equivalent to a group of complexes whose protein contents are reported differently by the different curated lists. We found 45 such groups and produced a consensus complex for each, resolving conflicts by a majority vote: a protein was included in the resulting complex only if it was found in more than half of the candidate complexes from the conflicted group. The remaining 18 nodes formed four connected components but no complete subgraphs, each component indicating non-transitive overlaps between three or more candidate complexes (e.g. A overlaps with B, and B overlaps with $\mathrm{C}$, but A does not overlap significantly with $C$ ). Manual inspection and consultation with experts resulted in 10 unique complexes being added to the reference list. The distribution of complex sizes in our reference set is shown in supplemental Fig. S2.

Constructing Positive and Negative Co-complex Protein PairsThe set of positive co-complexed protein pairs consists of all protein pairs that appear in the same complex in the reference set. For the negative set, we first consider all protein pairs $\left(P_{1}, P_{2}\right)$ such that $P 1$ is in a reference complex and $P 2$ is outside any version of that complex in any of the three hand-curated sets; we then exclude any pair that is within some other reference complex. The result of this process was 5065 positive pairs and about one million negative pairs.

Features for Predicting Co-complexed Relations-We constructed features for our protein-protein interaction network using five different data sources: the purification enrichment (PE) score from the consolidated network of Collins et al. (7), a cellular component from a

\footnotetext{
${ }^{3}$ Complex (dags.stanford.edu/Complex/).
} 
truncated version of the Gene Ontology (GO) (33), transmembrane proteins (31), co-expression (34), and yeast two-hybrid (Y2H) interactions $(35,36)$.

Our highest coverage source regarding protein-protein interaction comes from high throughput TAP-MS data of the Gavin et al. (3) and Krogan et al. (4) data sets. The recent work of Collins et al. (7) provides a coherent and systematic way of integrating the data from these separate assays into a high quality score that measures the probability of a protein pair to be co-complexed. The recent work of Hart et al. (5) provides a different integration method, but the results are quite similar, providing support for both of these procedures. We derived five features from the PE analysis: the direct score is computed based only on bait-prey information in the purifications; the indirect score is computed based on prey-prey information; the actual PE score is the sum of direct and indirect scores; the scaled score maps the PE score to a value between 0 and 1 to approximate the confidence value that the pair represents a true interaction; finally each protein is represented by a vector of its scaled PE scores with all the other proteins (where we assign its interaction with itself a score of 1 ), and we define our PE-distance feature as the cosine distance between the vectors of two proteins.

As the PE score provides most of the signals in predicting complexes (see "Results"), we only kept the 2390 proteins that have at least one scaled PE score above 0.2 with some other protein. Although this set only covers about $40 \%$ of the $\sim 6000$ yeast genes, it covers $81 \%$ of all protein members in the lists of high quality complexes that comprised our reference set. As noted earlier, we excluded proteins that appear exclusively in the four ribosomal subunits and the small nucleolar ribonucleoprotein complex. This resulted in the final list of 2195 proteins on which we performed our complex prediction.

Yeast two-hybrid assays also provide a direct measurement of protein-protein interactions. We derived these data from the assays of Ito et al. (35) and Uetz et al. (36). Interacting pairs are assigned a feature value of 1 . Pairs of proteins that appeared in the assay but were not observed to interact are assigned a feature value of -1 . All other pairs have 0 as their feature values.

The GO cellular component hierarchy (33) was downloaded on June 25,2007 . An examination of the hierarchy showed that many of the smaller categories (lower in the hierarchy) refer to particular complexes whose information is derived from the same small scale experiment that informs our reference set. Thus, to achieve a fair evaluation using the reference set, we removed categories of size less than 120 that can potentially contain the answer. The remaining 44 of 564 categories represent high level cellular localization information, much of which is obtained through high throughput experiments (37). Some sample categories include "endoplasmic reticulum part," "nuclear chromosome part," "mitochondrial membrane," and "cytoplasm."

We derived two pairwise localization features from the GO cellular component. One is the semantic distance measure (38), which is the log size of the smallest category that contains both proteins. However, this feature is a pessimistic assessment regarding the co-localization of the two proteins as lack of annotation of a protein in some category, particularly one that is a subset of its most specific category, does not necessarily mean that it cannot belong to this category. Therefore, we constructed a second feature, which is the log size of the smallest possible group that could contain both proteins (given the current evidence). It is computed in the following way between protein $A$ and protein $B$ whose most specific categories are $X$ and $Y$, respectively. If $X$ is a subcategory of $Y$, then the two proteins might belong together to any group if they were to be annotated with enough detail. Therefore, we use log of 120, the size of the smallest category, as our second feature. On the other hand, if $X$ and $Y$ are not subcategories of each other, we denote $Z$ to be the smallest common supercategory of $X$ and $Y$. We then denote $X^{\prime}$ (respectively $Y^{\prime}$ ) to be the category one level down the path from $Z$ to $X$ (respectively $Y$ ). Thus, assuming that $A$ and $B$ belong to the two different categories at $X^{\prime}$ and $Y^{\prime}$, the smallest semantic category that we can form that may contain them both is $X^{\prime} \cup Y^{\prime}$. Thus, our second feature is $\log \left(\mid X^{\prime} \cup\right.$ $\left.Y^{\prime} \mid\right)$.

A list of membrane proteins is obtained by parsing the transmembrane annotations in SGD (31). A pair of proteins is considered to be membrane if at least one of the proteins is found in the membrane. The first membrane feature is 1 if the pair is membrane and 0 otherwise. The second and third features are the product of the first feature with the direct and indirect PE score of the two proteins, respectively. This allows our boosting model to take into account the known fact that TAP-MS purifications work differently on membrane proteins from non-membrane proteins.

Microarray data were downloaded from the Stanford Microarray Database (34) on December 5, 2006; it contains a total of 902 experiments for yeast divided into 19 categories. The data were normalized to mean 0 and standard deviation 1 . We constructed a feature by computing the mean-centered Pearson correlation coefficient between the expression profiles of two proteins.

A final feature is obtained from small scale physical interactions. We downloaded protein-protein interactions from MIPS (8) and DIP (39) on March 21, 2006. We extracted from MIPS those physical interactions that are non-high throughput yeast two hybrid or affinity chromatography. For DIP, we picked non-genetic interactions that are derived from small scale experiments or verified by multiple experiments. This feature has a value of 1 for observed interactions and feature value 0 for all other pairs. Importantly there is a risk of cyclicity between these small scale interactions and the reference complexes. Therefore, to avoid a positive bias in our results, we omitted this feature in the cross-validation runs, which are evaluated against the reference complexes. For those runs that are trained on the entire set of reference complexes, this cyclicity is not a concern, so this feature was included. There are a total of 12 features for cross-validation runs and 13 features for runs that are trained on the entire reference set.

Integrating Multiple Features Using the LogitBoost-Boosting (22) is a class of algorithms that iteratively combines weak learners to give a representative ensemble. Each weak learner is a simple classifier, such as a decision stump, that may only weakly correlate with the labels. After a weak learner is trained, we add it to the ensemble with appropriate weight. In the next iteration, the algorithm puts more weights on the data points that are classified incorrectly by the current ensemble, which the next weak learner will focus on. Boosting is able to perform automatic feature selection and has accuracy that is better or comparable with other state-of-the-art classifiers such as support vector machines (40) in many domains. We implemented a version of boosting algorithms called LogitBoost (22) that uses decision stumps as weak learners and the logit function as the loss function. This variant is shown to be more robust to outliers and overfitting than the standard AdaBoost variant (41). Our experiments (data not shown) showed that this method performs well on our data compared with other versions of boosting and other classification algorithms such as logistic regression and support vector machines. The prediction of the learned ensemble classifier on a given protein pair is taken to be the affinity of the pair in the clustering algorithm below.

The HACO Algorithm - The standard HAC algorithm with average linkage (42) maintains a pool of merging candidate sets where the distance between two non-overlapping sets is as follows.

$$
d(A, B)=\frac{1}{|A \| B|} \sum_{P \in A, Q \in B} d(P, Q)
$$


In our setting, we take $d(P, Q)$ as the negative of the affinity between protein $P$ and protein $Q$. Note that $d(A, B)$ is the average of the edge distance between proteins in $A$ and proteins in $B$.

In $\mathrm{HAC}$, at each step, we pick the two non-overlapping sets with the closest distance, $A$ and $B$, and merge them to create a new set, $M$. $M$ is added to the pool, while the sets $A$ and $B$ are removed. Therefore in later steps, we could only consider the superset $M$ and would never be able to use $A$ or $B$ again to merge with some other set. Assume that there is another set $C$ whose distance to $A$ is only slightly larger than $d(A, B)$. In this case, the decision to merge $A$ with $B$ rather than with $C$ is arbitrary and unstable. When the actual clusters overlap, a more appropriate solution would be to have two overlapping merged candidates: $M=A \cup B$ and $N=A \cup C$. We adapt HAC to accommodate this intuition. We define the divergence between $A$ and $M$ as a measure of the cohesiveness of the set $M$ that is outside of $A$ (supplemental Fig. S3),

$$
\operatorname{divergence}(A, M)=\frac{1}{|E|} \sum_{(P, Q) \in E} d(P, Q)
$$

where $E$ is the set of pairs in $M$ but not in $A: E=\{(P, Q) \mid(P, Q) \in M \times$ $M-A \times A, P<Q\}$. (Here " $<$ " can be any ordering among the proteins, such as alphabetical, to avoid a pair appearing twice in the set $E$ ).

If $M$ is not overlapping with $C$, we have the choice of whether to use $A$ or $M$ to merge with $C$. If divergence $(A, N)$ - divergence $(A, M)$ is small, it makes sense to merge $A$ and $C$ to create a new set $N$ that is almost as coherent as $M$. On the other hand, if the difference is large, we would prefer to replace $A$ with its superset $M$ as the merging candidate to $C$.

In practice, we use $d(A, C)$ to approximate divergence $(A, N)$ : we check whether $\Delta=d(A, C)-\operatorname{divergence}(A, M)$ is small. Divergence $(A$, $N)$ is the weighted average of $d(A, C)$ and $d(C)$, the distance within $C$. $d(C)$ tends to be smaller than $d(A, C)$ because pairs within $C$, which is formed earlier by some merging, are more coherent than pairs between $A$ and $C$. Therefore, $d(A, C)$ tends to be smaller than divergen$\mathrm{ce}(A, N)$, so keeping $\Delta$ small is generally a more stringent requirement for ensuring that $N$ is almost as coherent as $M$. Moreover by forcing $d(A, C)$ to be small, we make sure the set $N$ is coherent not just because the distance within $C$ is small. With this consideration, we defined the modified distance between $A$ and $C$ (supplemental Fig. S3) as follows.

$$
d^{\prime}(A, C)= \begin{cases}d(A, C) & \text { if } \Delta<\rho \\ \infty & \text { if } \Delta \geq \rho\end{cases}
$$

The modified distance $d^{\prime}$ is used to pick the two closest sets to merge in the next iteration. If $\Delta$ is smaller than a margin, we make $d^{\prime}$ equal to $d$ and thus allow $A$ and $C$ to merge. On the other hand, if $\Delta$ is large, we make $d^{\prime}$ infinity and thus prohibit $A$ and $C$ from merging in favor of merging their supersets. $\rho$ is the margin parameter: the larger the margin $\rho$, the more likely a set $A$ is to be reused, resulting in more overlapping subsets constructed by the algorithm. If the margin is 0 , it reduces to the standard HAC. Therefore, our HACO algorithm is a generalization of the HAC. Note that we can eliminate a set from the merging candidate pool when its modified distances to all other sets are $\infty$. Of course we can define another modified distance as long as it is larger when $\Delta$ is large and close to $d(A, C)$ when $\Delta$ is small.

In practice, $A$ might have multiple supersets in the pool. Therefore, we look at all of the supersets of $A$ in the pool that are not overlapping with $C$ and use the set $M_{A, C}$ with smallest divergence from $A$, i.e. the one that provides the best replacement for $A$ in terms of the proposed merger with $C$.

$$
M_{A, C}=\underset{M \text { s.t. } A \subset M, C \cap M}{\arg \min } \operatorname{divergence}(A, M)
$$

We do the same thing with $C$ for its proposed merger with $A$.

$$
M_{C, A}=\underset{M^{\text {s.t. }} C{ }^{C} M, A \cap M=\phi}{\arg \min } \operatorname{divergence}(C, M)
$$

The smaller of divergence $\left(A, M_{A, C}\right)$ and divergence $\left(C, M_{C, A}\right)$ is used to compute the modified distance.

The algorithm terminates when there are no more non-overlapping sets to merge. The output is a cluster-lattice where the same cluster can be a child of multiple parents in the lattice. The lattice is cut at a certain threshold to generate a set of overlapping clusters. These predicted clusters are the sets that are still in the candidate pool when the distance in the merging process reaches the threshold.

Training and Test Regime-To evaluate our prediction accuracy against the reference set, we divided the 340 reference complexes into five disjoint subsets, or folds. As there are about a million negative pairs, for computational expediency, we randomly sampled onetenth of the negative pairs to be used in training while setting each negative pair to have 10 times the weight of the positive pairs.

For each fold in the 5-fold cross-validation, we hide one set and use the remaining four sets to train the affinity function for the protein pairs, the margin $\rho$ for the HACO, and the cutoff threshold for the resulting cluster-lattice. We use the same training set in all steps of our pipeline and evaluate the final predictions on complexes in a separate test set that is hidden during all steps of the training process. We select the cutoff threshold by maximizing the coverage (see below for the definition) on the training set. To pick the margin $\rho$, we cannot use coverage alone because our model would always prefer a bigger margin that keeps more sets in the pool. Therefore, we choose $\rho$ by maximizing the product of coverage and sensitivity (see below for the definition) on the training set. This approach trades off between the match with the reference set and the number of predicted complexes.

To evaluate our predictions against external data sources, such as biological coherence and essentiality, we augmented our model with a feature constructed from small scale physical interactions and trained it on the entire set of 340 reference complexes. To avoid circularity between features and evaluation, we did not evaluate the predictions from such runs against the reference complexes.

Evaluation Metrics for Matching between Predictions and Reference Complexes-The overlap between a reference complex $R$ and a predicted complex $C$ can be quantified in several ways (43) (supplemental Fig. S4).

$$
\begin{aligned}
& \text { Jaccard coefficient }=|R \cap C| /|R \cup C| \\
& \text { Hamming distance }=|R \cup C|-|R \cap C|
\end{aligned}
$$

We use both measures because of the size effect. For example, a Hamming distance of 2 between two large complexes, say both of size 5 , is a good match. In this case $\mathrm{JC}=4 / 6=0.67$. On the other hand, a Hamming distance of 2 between two small complexes of size 2 implies an overlap of only one protein, which could arise simply by chance. In this case $\mathrm{JC}=1 / 3=0.33$.

We define the coverage and sensitivity of a set of predictions so we can systematically evaluate genome-wide predictions. For each reference complex, we find the prediction that has the highest Jaccard coefficient. We define the scaled Jaccard coefficient (SJC) as follows: $\operatorname{SJC}(R, C)=\max \{0,2 \mathrm{JC}(R, C)-1\}$. We truncate the value at 0 because it may represent random overlap. In the above examples, the matching of the two large complexes of size 5 and Hamming distance 2 would have SJC $=0.33$, whereas the small ones of size 2 and Hamming distance 2 would have $\mathrm{SJC}=0$. We define the coverage as the average Jaccard coefficient per reference complex, 


$$
\text { Coverage }=\frac{1}{m} \sum_{i=1}^{m} \max _{j=1}^{n} \operatorname{SJC}\left(R_{i}, C_{j}\right)
$$

where $m$ is the number of reference complexes and $n$ is the number of predicted complexes.

For sensitivity, we sum the Jaccard coefficients of all the overlapping (reference and prediction) complex pairs and normalize by the total number of predicted complexes.

$$
\text { Sensitivity }=\frac{1}{n} \sum_{i=1}^{m} \sum_{j=1}^{n} \operatorname{SJC}\left(R_{i}, C_{j}\right)
$$

Biological Coherence of Predicted Complexes-We evaluate biological coherence of the predicted complexes using several metrics. The first is average semantic distance in the GO biological process hierarchy. GO data were downloaded on June 25, 2007. We computed the distance between two proteins as the log size of their smallest common category (38) as for the cellular component hierarchy above.

We downloaded the protein expression data from Ghaemmaghami et al. (44). We used log of measured protein levels in terms of molecules per cell as the protein abundance value.

The growth phenotype data were obtained from Hillenmeyer et al. (45). For each gene, its homozygous deletion strain is grown in 418 experiments with different drug treatments. The log ratio of the deletion strain's growth in no-drug control to its growth with the drug treatment is used to define the growth phenotype in that particular condition. For each pair of genes, we computed the Pearson correlation of the growth phenotypes across all 418 conditions; this is the measure used in the original study.

We downloaded the transcriptional regulation data from Maclsaac et al. (46) and Harbison et al. (47). We used a $p$ value cutoff at 0.001 and required conservation across species to define the transcription factors for each protein. We computed how many transcription factors are shared by any two proteins.

\section{Complex-Complex and Complex-Protein Interaction Prediction}

Constructing a Reference List of Positive and Negative ComplexComplex Interactions-We derived a reliable set of $S$. cerevisiae protein-protein interactions from MIPS (8) and DIP (39) downloaded on March 21, 2006. We extracted from MIPS those physical interactions that are non-high throughput yeast two hybrid or affinity chromatography. For DIP, we picked non-genetic interactions that are derived from small scale experiments or verified by multiple experiments. We computed the number of reliable interactions between proteins of two complexes and compared it with what we expect if the reliable interactions are distributed randomly. We define the two complexes to be interacting if the enrichment of reliable interactions is more than 20 standard deviations above the mean. Such strong enrichment is needed because the reliable interactions are very sparse, and the presence of even a very small number would result in a large deviation from the mean (e.g. for two complexes of size 2 and 5 respectively, we only need one reliable interaction of the total 10 pairs to get an enrichment of 10 standard deviations above the mean). We ended up with a list of 82 interactions between the set of 383 complexes we just predicted. To augment this list, we generated a list of 59 additional known interactions between 81 named complexes. To avoid the redundancy between those 81 named complexes and our 383 predicted complexes, we replace a predicted complex by a named complex if they overlap with $\mathrm{JC}>0.5$. This process gave us a total of 421 complexes with 133 unique interactions between them that are used as our positive reference set. We created a negative reference set of 3173 non-interactions by using all pairs of named complexes that are not in our positive set. The interaction status of all the remaining pairs of complexes, named or predicted, is treated as unknown.

For protein-complex interactions, in addition to the above negative set between complexes, we randomly sampled 6560 protein-complex pairs that are not in the positive set and added them to our negative reference set. The number 6560 was chosen so the ratio of positive to negative pairs for protein-complex interactions is the same as the ratio for complex-complex interactions. All our reference lists are available from our supporting Web site. ${ }^{3}$

Features for Predicting Interactions-Because there is no direct measurement of complex-complex or complex-protein interactions, we try to use as much indirect evidence as possible. Besides all data sources used for identifying complexes, we added four additional data sources based on correlation of growth fitness, correlation of transcription factor profile, protein-protein interaction prediction, and condition-specific expression correlation.

The correlation of growth fitness profile (45) is computed as described above under "Biological Coherence of Predicted Complexes." For each protein, we create a transcription factor (TF) profile vector where each position in the vector represents a TF and its value is 1 if the TF is found to regulate the protein (46) and 0 if it is not. We used the same transcription regulation data as described above under "Biological Coherence of Predicted Complexes." For any pair of proteins, we compute the mutual information between the profile vectors of the two proteins using the method described Date and Marcotte (48).

There are many works in integrating multiple sources of data to predict protein-protein interactions. In particular, the InSite method (49) integrates protein sequence motifs, evidence for protein-protein interactions, and evidence for motif-motif interactions in a principled probabilistic framework to make high quality predictions of proteinprotein interactions. Here we use the InSite method but trained without the reliable interactions between complexes in our positive reference set. We use the predicted probabilities that two proteins interact as one more data source.

Here we processed the expression data in accordance with our intuition that transient interactions occur under specific conditions, and we should only expect expression profiles of interacting proteins to be correlated only when at least one of the pair is active. Specifically we divided our expression data into 76 conditions (50-58), each of which represents a particular time course. In accordance with convention, we quantify the activity of a protein under certain condition according to its maximum deviation from norm, or in other words the maximum absolute expression (assuming norm to be 0). For each condition, we define a protein to be differentially expressed, or active, if its maximum absolute expression is above a cutoff, which we specify to be 1.0. For each pair of proteins, we compute Pearson correlation coefficient (PCC) separately in each condition. If a protein in the pair is inactive under a condition, the PCC value for the condition is assumed to be 0 . We use the PCC value, averaged across all conditions under which at least one protein of the pair is active, as our last feature type. Initial investigation showed that this feature is better correlated with the reference complex-complex interactions than the overall PCC across all conditions. We note that, for the task of predicting when two proteins are co-complexed, the simple correlation performed better (data not shown), consistent with the fact that the activity of two members of a stable complex is likely to be similar across a wide range of conditions.

Aggregating Signals between Proteins into Features between Complexes-All forms of evidence in our analysis involve a pair of proteins. To predict interactions between two complexes, $C$ and $D$, we aggre- 
gate the signals for all protein pairs between $C$ and $D$ and produce the following features,

$$
f_{i j}=A_{i}\left(\left\{S_{j}(P, Q) \mid P \in C, Q \in D\right\}\right)
$$

where $\left.A_{i}\right)$ is some aggregating function, such as sum, maximum, mean, minimum, decayed maximum, decayed minimum, etc. (See supplemental Table 1 for a complete list of aggregating functions and their definitions.) $S_{j}$ represents the $j$ th feature type between a pair of proteins. We also use four global features, independent of the data sources: size of the first complex, size of the second complex, number of protein pairs between the two complexes, and number of overlapping proteins between the two complexes. The features for interactions between a protein $P$ and a complex $C$ are identical except that we only need to aggregate the signals over all pairs $(P, Q)$ for $Q$ in $C$.

The naïve Bayes model that we use assumes all features to be conditionally independent of each other given the status of whether two complexes interact or not. Therefore for each data source, we pick only the best aggregating function to reduce the conditional dependences between the features. To do this, we define $r_{i j}$ to be the area under the ROC curve if we use the feature $f_{i j}$ alone to predict complex-complex interactions: the greater $r_{i j}$, the stronger the correlation between the feature and the presence of a complex-complex interaction. Therefore, for naïve Bayes, we use, for each feature type $j$, the feature $f_{j}=f_{i j}$ where $i$ gives rise to the maximum value $r_{i j}$. Supplemental Table 2 lists the aggregating function chosen for each feature type.

Learning and Predictions-We experimented with different machine learning algorithms for making our predictions: 1) a simple naïve Bayes model where the effects of different feature types are assumed to be independent, 2) a discriminative boosting algorithm as we used in predicting co-complexed affinities between protein pairs above, and 3) a naïve Bayes model where the unlabeled complex-complex interactions are taken to be unobserved variables, and the model is trained via the expectation maximization (EM) algorithm. This last approach is based on the fact that the amount of labeled training data is quite limited in this task, but the unlabeled data also provide us with useful information about the behavior of different features in interacting and non-interacting pairs. A variant of this same approach was used with success in the InSite model (49).

More formally, for each pair of complexes, we construct an "interaction variable" whose value is 1 if the two complexes are in the positive reference set of interacting complexes, 0 if they are in the negative reference set, and unobserved otherwise. Each feature of the complex pair is associated with two conditional distributions: one for the case of an interacting pair and the other for the case of a non-interacting pair. These distributions are defined via some parametric class (see supplemental Table 3). The distributions for the different features are taken to be independent of each other within each of the two cases. The model is trained via the following EM procedure. We initialize the model parameters to those that would be obtained from maximum likelihood estimation using the pairs in our reference set alone. We then iteratively repeat the following two steps until convergence. In the E-step, we use our current model to compute the marginal probability of each unobserved interaction variable given the features associated with the pair. We use the computed probability as a soft assignment to the interaction variable. In the M-step, we learn the parameters for the distributions using maximum likelihood estimation based on the inferred soft assignment to all interaction variables; the variables in the reference set are always fixed to their known value. We use the model obtained at convergence to predict, for each pair of complexes not in our reference set, the probability with which the pair interacts.

We used the same naïve Bayes + EM procedure when making predictions using only one of the features (PE score or InSite proba- bility), which we used as a comparison base line. In these comparisons, we used the same aggregator selected for the model using all the features.

When training using the LogitBoost model, we are not making independence assumptions between the different features. Hence there we include all features $f_{i j}$ instead of just picking the best aggregating function for each feature type. We used the same naïve Bayes + EM procedure for the protein-complex interaction predictions, although the best aggregating functions picked and the set of parametric classes used for the feature distributions were a little different. (See supplemental Tables 2 and 4.)

Functional Coherence of Complexes Predicted to Interact-We evaluate whether two interacting complexes are more likely to share the same functional category. We used functional categories from MIPS (8), which has 18 functional categories with an average of 684 proteins per category. A complex is assigned to a particular functional category if more than half of its components belong to the functional category. We only perform our evaluation on complex pairs where both complexes are assigned to some MIPS functional category.

\section{Experimental Validation}

TAP Purification-Two liters of yeast culture expressing Pil1-TAP was grown to $A_{600}=0.8$ and subsequently harvested. The resulting pellet was resuspended in $8 \mathrm{ml}$ of buffer $A$ (150 mM potassium acetate, $20 \mathrm{~mm}$ HEPES, $\mathrm{pH}=7.4,2 \mathrm{~mm}$ magnesium acetate, $5 \%$ glycerol) and frozen in liquid nitrogen. Total proteins were extracted by bead milling of the frozen pellet followed by addition of Triton $\mathrm{X}-100$ to $1 \%(\mathrm{w} / \mathrm{v})$ final concentration. Solubilized extracts were cleared by two centrifugations of $4 \mathrm{~min}$ at $4000 \times g$ and incubated with IgG-Sepharose for $2 \mathrm{~h}$. Beads were washed six times with $50 \mathrm{ml}$ of buffer B (150 mM potassium acetate, $20 \mathrm{~mm}$ HEPES, $\mathrm{pH}=7.4,2$ $\mathrm{mm}$ magnesium acetate, $5 \%$ glycerol, $1 \%$ Triton X-100). Proteins were eluted by tobacco etch virus protease cleavage in $200 \mu \mathrm{l}$ of buffer for $2 \mathrm{~h}$ and analyzed by SDS-PAGE by Coomassie staining. Bands were cut and digested with trypsin, and peptides were extracted and analyzed by LC-MS/MS as described previously (59).

EMAP Experiments-EMAP experiments and subsequent data analysis were done as described previously $(60,61)$. Data from these experiments are presented on our supporting Web site. ${ }^{3}$

\section{RESULTS \\ Method Overview}

We compiled a reference set of complexes by combining literature-derived results from small scale experiments in MIPS (8) and SGD (31) with a hand-curated list (see our supporting Web site $^{3}$ ) that we generated. The MIPS, SGD, and hand-curated sets contain 225, 195, and 164 complexes, respectively (supplemental Fig. S1a). We established correspondence between the three lists and combined them into a high confidence reference set suitable for training our method and for evaluating the accuracy of its predictions (see "Experimental Procedures"). This curated set was compiled prior to the development of our method and was not subsequently revised.

We then formulated the task of predicting whether two proteins were members of the same complex as a machine learning task. We used our reference set to construct a high quality set of positive and negative examples. We constructed features that are useful for predicting this relationship from 
five different data sources: the PE score from the consolidated network of Collins et al. (7), a cellular component from a truncated version of the GO (33), transmembrane proteins (31), co-expression (34), and $\mathrm{Y} 2 \mathrm{H}$ interactions $(35,36)$ (see "Experimental Procedures"). We then applied the boosting algorithm (22) for training the predictor. Boosting was selected because of its high accuracy, robustness to outliers, and ability to perform automatic feature selection. The prediction of the boosting classifier on a given protein pair is taken to be the affinity of the pair in the clustering algorithm below.

Our initial experiments showed that HAC, which progressively merges sets of proteins with strongest affinity, produces the best results for complex reconstruction if trained to optimize for that task. However, HAC has several significant limitations. First, it does not allow clusters to overlap, whereas actual complexes do share subunits. Second, it uses a single cutoff to decide the granularity of the complexes constructed. A cluster near the cutoff in the dendrogram can be formed even if it is the result of merging two relatively weakly connected subclusters $A$ and $B$. Such a cluster, although of lower confidence, still excludes both $A$ and $B$ from being predicted as a complex; this occurs even if $A$ and $B$ are strong candidates for being a complex. Finally once a set of proteins is merged with another set, it cannot merge with anything else even if the affinity is only slightly lower. Therefore an incorrect decision cannot be fixed later in the process.

To address these limitations, we constructed a novel clustering algorithm called HACO that allows a set of proteins to be merged with multiple other sets with which it has comparably strong affinity (see "Experimental Procedures"). HACO addresses all of the limitations above. First, it produces clusters that can overlap. Second, when merging $A$ and $B$ into a single cluster $\mathrm{C}$, it also has the option of leaving $\mathrm{A}$ and/or $\mathrm{B}$ as candidate complexes, avoiding a wrong decision because of an arbitrary cutoff. Finally as it allows the same cluster to be used in multiple places, it avoids many mistakes that arise from an almost arbitrary breaking of near ties. Both our boosting algorithm and the HACO code are freely available on our project Web page, ${ }^{3}$ allowing them to be used for predicting complexes with other forms of data.

\section{Complex Predictions}

Coverage and Sensitivity of Predicted Complexes-We compiled a reference set of complexes from MIPS (8), SGD (31), and hand-curation (see our supporting Web site ${ }^{3}$ ) that is more comprehensive than previous studies $(5,6)$. Although it still contains noise and bias, it provides us with the ultimate evaluation of our predictions. There are 340 complexes in our reference set with an average of 4.9 proteins per complex (supplemental Fig. S1b).

To predict complexes, we first trained our model to predict pairwise co-complex interactions and then used our HACO algorithm to cluster the resulting pairwise affinity network into complexes. We constructed features for our protein-protein interaction network using five different data sources: the PE score from the consolidated network of Collins et al. (7), a cellular component from a truncated version of the GO (33), transmembrane proteins (31), co-expression (34), and $\mathrm{Y} 2 \mathrm{H}$ interactions $(35,36)$. We tested our approach using a standard 5 -fold cross-validation regime, training on $80 \%$ of the complexes and testing on the remaining $20 \%$; the test set was not used in any aspect of the training of the model. For each fold in the 5-fold cross-validation, we applied HACO to the affinity measure learned using the boosting model on the training data. We evaluated the resulting clusters on the hidden test set. We predicted 417.8 complexes per fold with at least two proteins for each complex. Each complex contains 4.30 proteins on average (supplemental Fig. S1).

We define a complex to be well predicted if it is within Hamming distance (see "Experimental Procedures") of 2 to some predicted complex. However, two small complexes can be quite different even if their Hamming distance is 2. Therefore we also require the Jaccard coefficient (see "Experimental Procedures"), which takes into account the size of the complexes, to be above 0.5 . We also measure the coverage and sensitivity of the set of predictions (see "Experimental Procedures"): coverage measures how well the reference set is covered by our predictions, and sensitivity measures how well each predicted complex overlaps with the reference set, a measure that takes into consideration the number of predicted complexes.

We compared our results with those of Bader and Hogue (62), Gavin et al. (3), Krogan et al. (4), Hart et al. (5), and Pu et al. (6). As we discussed, each method made different decisions for defining the affinity function and for clustering it. Bader and Hogue (62) used a novel clustering algorithm called molecular complex detection (MCODE) to detect densely connected regions in the protein-protein interaction network. Gavin et al. (3) computed a socioaffinity score between each pair of proteins that compares the number of times the two proteins are observed together in some purifications relative to what is expected by chance. The pairwise network of socioaffinity scores is then subjected to a procedure that produces overlapping clusters. Complexes are composed of a "core" that appears in most runs of the clustering algorithm and "attachments" that appear only in some. Most of the recent methods appear to have converged on using the $\mathrm{MCL}$ algorithm (9) albeit on different affinity functions. Krogan et al. (4) used a machine learning approach, trained on MIPS reference complexes, to predict the confidence that a pair of proteins is in the same complex. Hart et al. (5) defined a $p$ value by comparing observed relative to expected number of interactions applied to three sets of purifications (2-4). Pu et al. (6) applied MCL directly to the PE score of Collins et al. (7). All of these MCL-based methods produce non-overlapping clusters, although the method of Pu et al. (6) used a postpro- 
cessing phase to identify proteins that are likely to be recruited by multiple complexes.

Fig. 1a shows the accuracy of our method in reconstructing the reference complexes as compared with the best of these other approaches. As we can see, our affinity score achieves significantly better results than any of these methods; the results are better even when we use simple HAC for the clustering and improve further when we use HACO. We note that Hart et al. (5) and Pu et al. (6) are the state of the art in complex predictions and have been extensively compared with other complex prediction methods. In particular, Pu et al. (6) applied MCL to the same set of PE scores (7) as we used. HACO was able to perfectly recover 42 and $46 \%$ more reference complexes compared with Hart et al. (5) and Pu et al. (6), respectively ( $p$ values $<0.01$ ). The corresponding increase in sensitivity is 6 and $29 \%$, respectively, and increase in coverage is 28 and $33 \%$, respectively. The results suggest that these improvements are a consequence of our use of data integration with state-of-the-art machine learning. In particular, the Pu et al. (6) method and the Hart et al. (5) method, both of which used MCL applied to different affinities obtained from the TAP-MS data, performed very similarly. Interestingly HAC applied to the PE score performed slightly better than MCL applied to the PE score (HAC PE versus Pu et al. (6)). These three methods performed better than those of Bader and Hogue (62), Gavin et al. (3), and Krogan et al. (4) likely because of the fact that these earlier methods used only a single set of purifications. These results demonstrate the importance of combining data from multiple data sources integrated appropriately. We note that MIPS complexes are used, albeit in a very limited way, in generating the PE score. To avoid any risk of circular reasoning, we ran the same experiments using the SGD complexes alone as an independent reference set; the results (supplemental Fig. S2a) show that the improvement of our method over others remains consistent in this reference set as well.

The HACO algorithm helps address several of the limitations of the HAC approach. First it reduces the sensitivity of the complex definitions to a single universal threshold in the hierarchy. One such example involves the 15-protein SAGA complex. Here HAC predicts a 24-protein superset of the SAGA complex. This cluster is a much weaker cluster than SAGA itself: the average affinity between the SAGA proteins is 0.35 as compared with the average affinity of -1.19 for pairs within the 23 proteins excluding pairs of SAGA proteins. By comparison, $\mathrm{HACO}$, by keeping multiple hypotheses relative to the cutoff, predicted a 23-protein cluster (similar to the HAC prediction) but also predicted the subcluster that corresponds perfectly to the SAGA complex. The second limitation addressed by HACO is that it avoids an early commitment to incorrect outcomes. For example, the affinity between Rad23 and Png1 is slightly higher than that between Rad23 and Rad4. HAC incorrectly merges Rad23 and Png1 and now cannot reuse Rad23 in any other complex. HACO can reuse
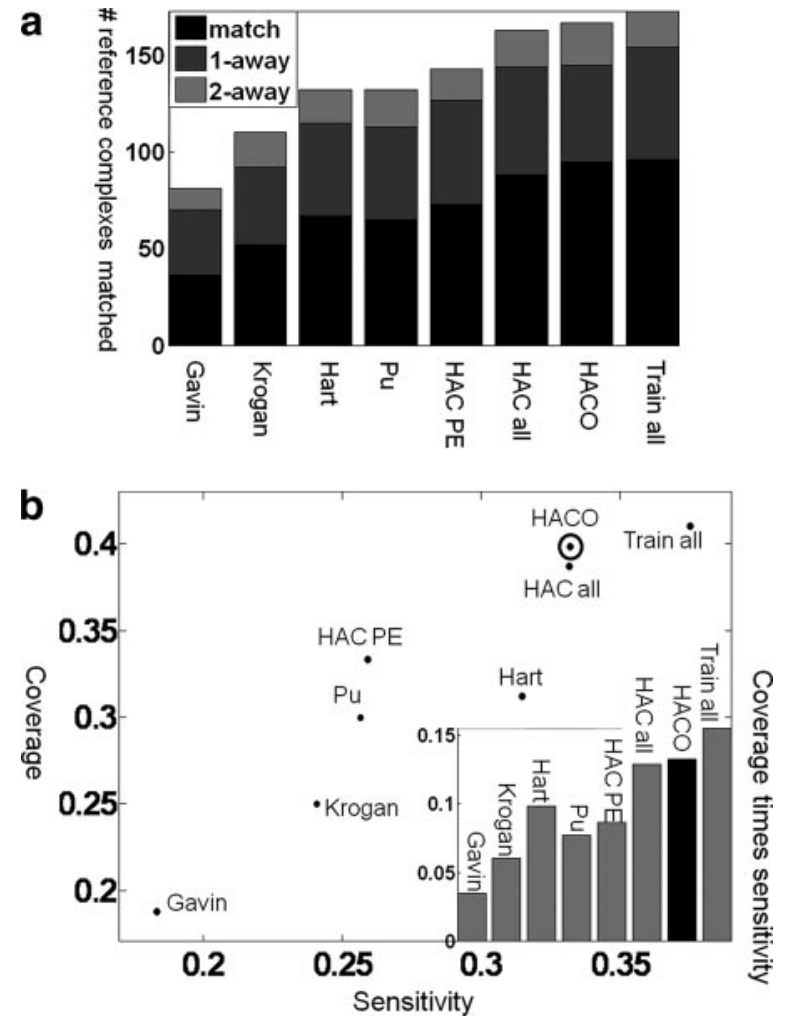

FIG. 1. Accuracy in reconstructing reference complexes. A comparison of predicted complexes to other state-of-the-art methods in the ability to accurately reconstruct reference complexes is shown. $a$, the number of reference complexes well matched by our predictions ( $y$ axis) and for the different methods we compared ( $x$ axis). The prediction quality is shown as bars: black, perfect prediction; dark gray, predictions that differ by a single protein (one extra or one fewer); light gray, predictions that differ by two proteins. Hart et al. (5) and Pu et al. (6) are state-of-the-art methods that outperform Gavin et al. (3) and Krogan et al. (4). The method of Bader and Hogue (62) has even lower accuracy (data not shown). Applying HAC to PE score (HAC PE) performed slightly better than Hart et al. (5) and Pu et al. (6), which use MCL. Our model, which uses LogitBoost and clustering, is able to achieve significantly better results than any other method by integrating multiple sources of data. The results are better even when we use simple HAC (HAC all; 88 perfect matches) for the clustering and improve further when we use HACO (95 perfect matches). This improvement is consistent over all five folds in our cross-validation process: over the five folds, HAC PE recovers 15, 11, 16, 22, and nine of the complexes; HAC all recovers 21, 13, 21, 23, and 10; and HACO recovers $24,13,23,23$, and 12 . This consistency over folds demonstrates the robustness in the improvement we obtain using our method. In "Train all," we trained on all data and tested on the same data; this method achieves only slightly higher accuracy, which indicates little overfitting to the training data and supports evaluating biological coherence of our predictions on this set. $b$, the $x$ axis is the sensitivity of our predictions, which quantifies how likely a prediction is to match some reference complexes; the $y$ axis is the coverage of our predictions, which quantifies how many reference complexes are matched by our predictions (see "Experimental Procedures"). Our approach has higher sensitivity and coverage than other methods. HACO has the highest product of sensitivity and coverage except for Train all, which trains and tests on the same data and thus provides an unachievable upper bound on performance. 


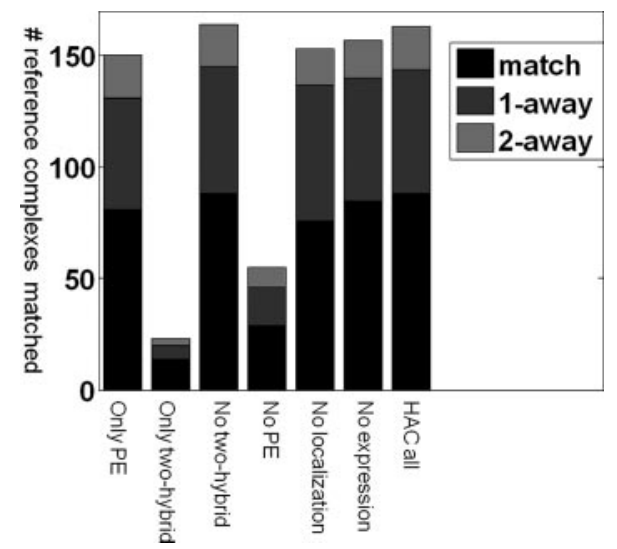

FIG. 2. Contribution of each data source. To assess the contribution of each data source, we successively applied our pipeline with HAC to each data source alone and to all data sources except one; shown are the interesting cases (see also supplemental Fig. S2b) using the same format as in Fig. 1. The PE score by itself predicts most of the complexes, but we still get a significant improvement by integrating other data sources. Localization or expression are nonspecific and by themselves do not predict any complexes at all, but removing them decreases the accuracy, suggesting that they help clarify ambiguities in the TAP-MS data. Conversely the yeast twohybrid feature by itself predicts a reasonable number of complexes, but removing it does not decrease accuracy at all, suggesting that it is redundant with the TAP-MS data.

Rad23, merging it with Rad4 to create a complex that perfectly matches the NEF2 (nucleotide excision repair factor 2) complex in the reference set.

Contribution of Each Data Source for Predicting Complexes-Given the importance of data integration, it is useful to see which data sources play the most important role in our results. We first considered the contribution of each feature to our learned affinity function. Our approach uses LogitBoost (22), which defines the affinity function as the weighted sum of many weak learners, each of which is a decision stump on one of the features. The top weak learners involve features that are deemed to be most predictive. The top features in the order of their importance are: correlation of PE score (weight, 3.84); semantic distance in the truncated GO cellular component categories (-2.2); direct PE score, which is based only on direct bait-prey interactions (0.58); small scale physical interactions (0.55); and co-expression (0.16). It is interesting to note that the correlation of the PE score is deemed more informative than the PE score itself. One explanation is that the pairwise $P E$ score between proteins $P$ and $Q$ is still a noisy measure for co-complexness, but if $P$ and $Q$ are truly cocomplexed, they are likely to have similar interactions with other proteins.

As another metric for assessing the importance of each data source to the quality of our predictions, we successively applied our pipeline with HAC to the data source alone and to all data sources except that data source (Fig. 2 and supplemental Fig. S2). The PE score plays the dominant role and by itself predicts most of the complexes. Importantly our method here combines different variations of PE score (direct, indirect, scaled, total, and correlation) using boosting, generating an affinity score that is quite a bit better at predicting complexes than the original scaled PE score (73/54/16 perfect matches/one away/two away for HAC PE in Fig. 1a versus 81/50/19 for the PE-based features alone in Fig. 2). This result demonstrates the value of applying machine learning methods specifically optimized for the problem of complex identification. Nevertheless we still get a significant improvement by integrating in other data sources.

Localization and expression have a similar effect. By itself, neither predicts any complexes at all; this is not surprising, as both are features with low precision. However, removing each of them decreases the accuracy, suggesting that they provide a signal that is independent of the PE score, and can help resolve some of its ambiguities and errors. The yeast twohybrid feature has the opposite behavior: in isolation, it predicts a reasonable number of complexes; however, removing it does not decrease accuracy at all. This behavior can be explained by the hypothesis that yeast two-hybrid data largely correlate with PE score; thus, although the feature is predictive, it does not add much given the PE score data. This last hypothesis is further verified by the fact that localization and expression features appear within the top five weak learners, whereas yeast two-hybrid feature does not.

Biological Coherence of Predicted Complexes-Having tested the ability of our method to reconstruct reference complexes, we produced a final set of predictions from our method. Here we train on all reference complexes and introduce an additional feature relating to interaction in small scale experiments; this feature was not used in the comparison with reference complexes to avoid potential circularity between this feature and the definition of the reference complexes. Overall this process resulted in 383 predicted complexes, which can be found on our supporting Web site. ${ }^{3}$ We evaluated the validity of these complexes by comparing with external data sources not used in the training and not directly related to reference complexes. For all biological coherence validations, we compute the coherence for each complex as the average of the coherence measure for all pairs in the complex. Then we take the average across all complexes predicted. We compare with the methods of Hart et al. (5) and $\mathrm{Pu}$ et al. (6), which consistently out-performed all previous methods. As a different benchmark, we also compare with the coherence for the highest affinity protein pairs (those that are most likely to belong to the same complex).

We validate our predictions by looking at various measures of biological coherence (Fig. 3): similarity of GO biological process, similarity in the level of protein abundance for different complex components, correlation of growth defect profiles across a broad range of conditions, and co-regulation as measured by sharing of transcription factors. For all measures, HACO with our affinity function considerably outperformed all other approaches with the method of Hart et al. (5) 


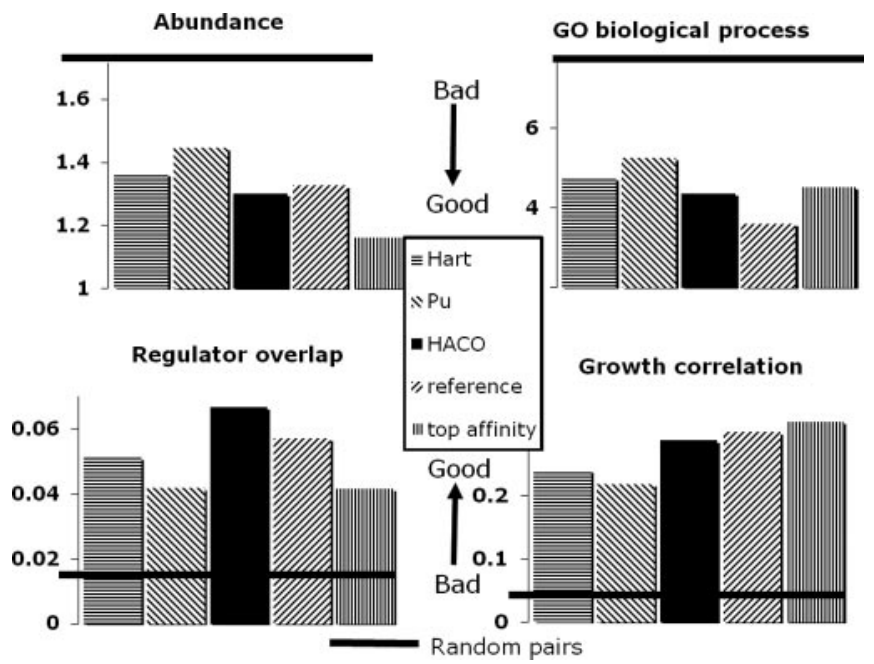

FIG. 3. Coherence of our predicted complexes. We computed the functional coherence between proteins in the same complex against external data sources that are not used in training. More coherent complexes have a smaller difference in protein abundance, have a smaller semantic distance in GO biological process, share more transcriptional regulators, and have a higher growth fitness correlation. The $y$ axis shows the values for these metrics of functional coherence; also shown is the performance of random pairs (thick horizontal line). Our predicted set of complexes significantly outperforms other state-of-the-art methods. For GO biological processes, our complexes have a semantic distance 8 and $17 \%$ lower than the methods of Hart et al. (5) and Pu et al. (6), respectively. For protein abundance, the improvement over Hart et al. (5) and Pu et al. (6) is 5 and $10 \%$, respectively; conversely our complexes are $12 \%$ less coherent than the top affinity pairs, suggesting that proteins with lower affinity scores can be members of the complex but also play other roles in the cell, reducing their correlation with other proteins in the same complex. For the correlation of growth phenotypes across different conditions, our predicted complexes are 19 and $31 \%$ more coherent, respectively, a very significant improvement. Finally protein pairs within our complexes on average share 30 and 59\%, respectively, more transcription factors than those of Hart et al. (5) and Pu et al. (6). The comparison with the reference complexes shows that our complexes are considerably more coherent on regulator overlap and perform similarly on correlation of abundance and growth phenotype. Conversely our complexes are $21 \%$ less coherent than the reference complexes on GO biological process annotations; this is not surprising as the reference complexes and GO annotations are derived (at least in part) from similar data sources, such as literature and small scale experiments.

being the closest competitor. Most striking were the improvements in correlation of growth phenotypes across multiple conditions and in coherence of the transcriptional regulation program. To specifically test our complex formation process, we also compared pairs of co-complexed proteins with pairs that have high affinity (as computed by our boosting algorithm). The results were largely comparable with the notable exception of protein abundance where our complexes are $12 \%$ less coherent than the top affinity pairs; this suggests that proteins with lower affinity scores can be members of the complex but also play other roles in the cell, reducing their correlation with other proteins in the same complex. The comparison with the reference complexes is also interesting. Our complexes are considerably more coherent than the reference complexes on regulator overlap and perform similarly on correlation of abundance and growth phenotype. Conversely our complexes are significantly less coherent than the reference complexes on $\mathrm{GO}$ biological process annotations; this is not surprising as the reference complexes and GO annotations are derived (at least in part) from similar data sources, such as literature and small scale experiments. Overall when comparing with data sources that were not used in constructing the reference complexes, our predictions seem to perform as well or better than the reference set, suggesting that our predictions provide a strong set of complexes that can be used as a new reference.

In-depth Study of Predicted Complexes-We also did a systematic, manual evaluation of many of our predicted complexes. We first considered the complexes that were one away from the reference set, that is a protein $\mathrm{P}$ and a complex $A$ where $P$ was either added to $A$ or removed from $A$ in contradiction to the reference set. Most of these cases represented situations where it is unclear whether $P$ really did belong in $\mathrm{A}$ or not, and different biologists often have different opinions. For example, the Torpedo complex, which is involved in transcriptional termination by RNA polymerase II (63), was reported to be comprised of three subunits: the exonuclease Rat1, Rai1, and Rtt103. We predicted that Rtt103 was not a component of this complex, consistent with the weaker stoichiometric association of Rtt103 with the two other tightly associated members of the complex (63). In another example, we predicted that Csn12 was not a component of the COP9 signalosome, which is involved in deneddylation (27). Consistent with this, Maytal-Kivity et al. (64) demonstrated that Csn12 is the only component of this complex that is not required for the deneddylation activity. Furthermore we found that Csn12, but not other signalosome subunits, is required for efficient mRNA splicing at a number of genes in budding yeast, ${ }^{4}$ suggesting that Csn12 plays multiple cellular roles and may not be an integral member of the complex. Finally we predicted that Ski7 is part of the exosome complex, which harbors $3^{\prime}$-to-5' exonuclease activity and acts on many different types of RNA. Evidence suggests that Ski7 acts as an adaptor to target the exosome to mRNAs lacking stop codons (65).

In other cases, however, the predictions made by our algorithm were interesting and worthy of further investigation. One such example is the eisosome, previously described to be primarily comprised of two subunits (Pil1 and Lsp1) (25);

\footnotetext{
${ }^{4}$ Wilmes, G. M., Bergkessel, M., Bandyopadhyay, S., Shales, M., Braberg, H., Cagney, G., Collins, S. R., Whitworth, G. B., Kress, T. L., Weissman, J. S., Ideker, T., Guthrie, C., and Krogan, N. J. (2008) A genetic interaction map of RNA-processing factors reveals links between sem1/Dss1-containing complexes and mRNA export and splicing Mol. Cell 32, 735-746
} 
a

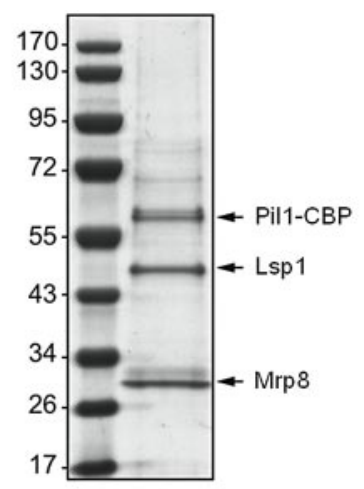

b

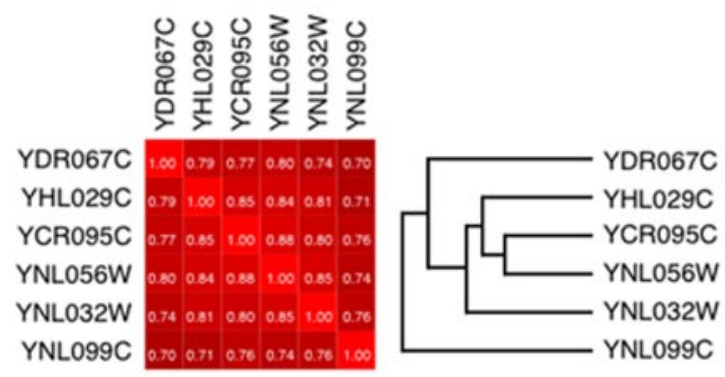

$\begin{array}{lllllll}0.0 & .17 & .33 & .50 & .67 & .83 & 1.0\end{array}$

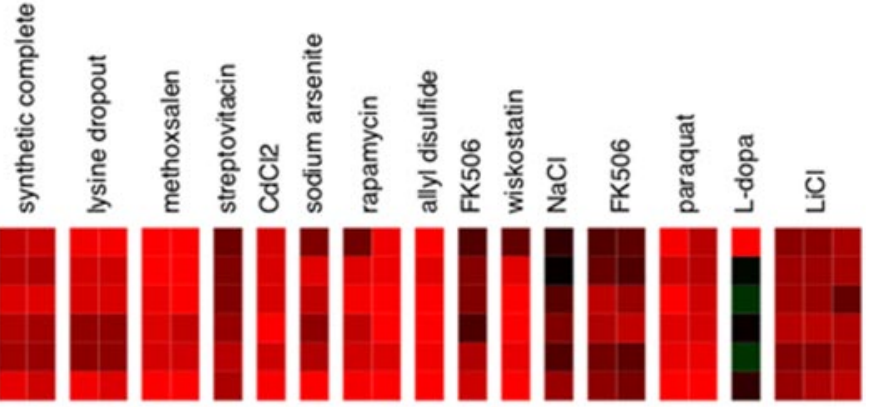

YNL032W

YNL099C
C

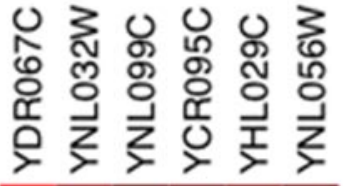

YDR067C 1.000 .540 .330 .380 .460 .39

YNL032W $0.54 \quad 1.00 \quad 0.40 \quad 0.45 \quad 0.45 \quad 0.43$

YNL099C $0.330 .40 \quad 1.00 \quad 0.530 .430 .46$

YCR095C $0.380 .45 \quad 0.53 \quad 1.000 .54 \quad 0.48$

YHL029C

YNL056W

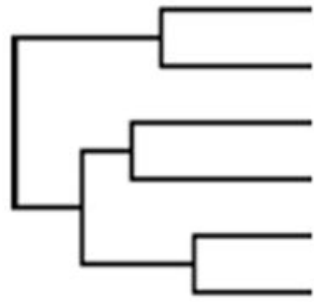

YDR067C

YNL032W

YCR095C

YHLO29C

YNL056W

YNL099C
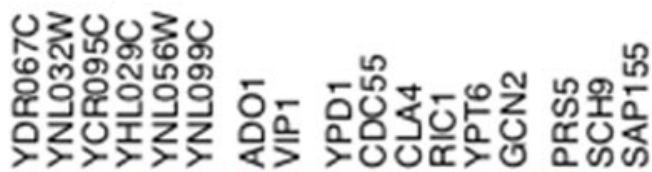

年
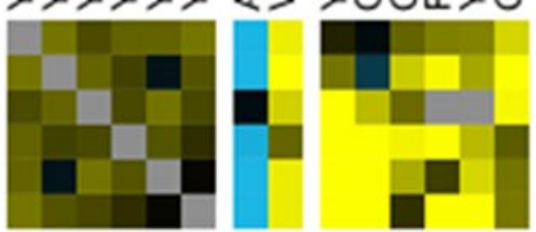

œळ

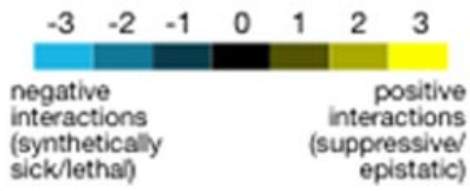

FIG. 4. Validation of novel biological findings. a, Pil1, Lsp1, and Mrp8 form a stable complex. TAP-tagged Pil1 was affinity-purified from yeast. Highly enriched fractions were run on SDS-PAGE, and co-purifying proteins were identified by LC-MS/MS as Lsp1 and Mrp8, indicated on the left; protein sizes are shown in $\mathrm{kDa}$ on the right. The result supports our prediction that Mrp8 is a component of the eisosome complex. $b$ and $c$, support for newly uncovered NSC complex comprised of six genes (YCR095C, YHL029C, YNL032W, YNL056W, YNL099C/OCA1, and YDR067C), four of which are phosphatases. Five of these components were predicted by HACO to be a stoichiometrically stable complex; based on other data (shown in this figure) we conjecture that the sixth (YDR067C) may also be a member of this complex. $b$, support in chemical genomics data of Hillenmeyer et al. (45), which measured the fitness profiles of all non-essential homozygous yeast mutants under 418 conditions. Left, the fitness profiles of the six predicted NSC members cluster tightly together. Right, shown are the conditions in which at least one of the six components had a fitness defect with $p<1 e-10$; the consistently strong sensitivity to rapamycin, lysine dropout, and synthetic complete medium suggests the involvement of these proteins in response to nutrient starvation. $c$, support in new EMAP data, which measured quantitative genetic interaction profiles with $\sim 500$ genes involved in signaling. Left, the genetic interaction profiles of the six components cluster tightly together. Right, the components have positive genetic interactions among them and exhibit significant interactions with genes involved in nutrient starvation response, including Sch9 and Gcn2. CBP, calmodulin-binding peptide. 
however, we predicted that the complex contains another, previously undescribed component, Mrp8. Consistent with this prediction, TAP purification of Mrp8 reveals that it is indeed a stoichiometrically stable member of the eisosome complex (see Fig. 4a). Further work will be required to determine the role that this novel subunit plays in eisosome function.

We also studied the novel complex predictions, those that did not match any of the reference complexes above our match threshold. A number of these turned out to be well characterized complexes that, for some reason, had not (yet) been included into any of our three reference sets. For example, we identified the Sit4/Sap185 heterodimer phosphatase complex (66); a complex comprised of Yos9, Hrd3, Usa1, and Hrd1 that is involved in endoplasmic reticulum-associated degradation (67, 68); and the U3-processome complex (complex 1129) involved in the generation and regulation of the small ribosomal particle (69). Many others comprised plausible complexes that, to our knowledge, have not yet been characterized and are worthy candidates for further investigation.

One such example is a complex (complex 1014) comprised of five components (YNL099C/OCA1, YNL056W/OCA2, YNL032W/SIW14/OCA3, YCR095C/OCA4, and YHL029C/ OCA5), four of which are putative phosphatases. One of the proteins (Oca1) has been previously shown to be required for cell cycle arrest in response to exposure to a lipid peroxide (70). We note that the individual pairwise connections between these proteins were observed before and that various forms of evidence support their shared function (14), including a shared phenotype of oxidant-induced cell cycle arrest, which underlies the current name of many of these genes in SGD. However, this group was not previously identified as a complex nor was its function characterized. Further supporting our prediction of this group as a complex is the fact that the chemical-genetic interaction profiles of the five genes were tightly clustered in a recent high throughput study (45) (Fig. 4b). Mutations in the components of the complex resulted in significant sensitivity to a number of conditions, including several that are related to nutrient starvation, including exposure to rapamycin, lysine dropout, and synthetic complete medium. To further characterize the functions of these factors, we subjected the mutants to quantitative genetic interaction profiling using an $\operatorname{EMAP}(60,61,71)$ focused on genes implicated in signaling, including protein and small molecule kinases and phosphatases (see our supporting Web site $\left.^{3}\right)$. Again we found that the components of the complex had strong positive genetic interactions between them and clustered tightly together within the set of $\sim 500$ genes included in the EMAP, both factors that indicate a strong functional connection (60). Specifically we found that all components have strong negative genetic interactions with Sch9, the yeast homolog of S6 kinase and a central node in nutrient signaling $(72,73)$. Conversely we found strong positive genetic interactions with GCN2, a protein kinase that phosphorylates the $\alpha$ subunit of translation initiation factor elF2 (Sui2) in response to nutrient starvation (74). Collectively these data suggest an involvement of these proteins in response to nutrient starvation. Interestingly both the chemogenomic profiling and the genetic interactions suggested a functional connection to another uncharacterized gene, YDR067C, which may form a sixth member of the complex. Based on the coherence of this complex and its strong links to nutrient starvation, we propose to name this six-protein complex NSC.

The predictions made by our algorithm also contained a number of mistakes, which fell into two main categories. The first comprised subsets of known complexes, such as subsets of the pre-60 S ribosomal particle (complexes 1088 and 1106). These may represent functionally distinct submodules within larger complexes and therefore may provide useful insight about complex structure. Consistent with this notion, we identified the deubiquitination unit of SAGA (Ubp8/Sgf11) (75-77). The other category of error involved pairs of complexes that either interact or share subunits and were merged by the HACO procedure into a single complex. For example, complex 1125 is comprised of two chromatin-remodeling complexes, INO80-C and SWR-C, which have shared components, including Rvb1 and Rvb2, members of the RuvB family of helicases (78). These two error modes illustrate the difficulty in selecting the appropriate granularity for making complex predictions where some complexes occur fairly low in the clustergram so that they have very high affinity with components outside the complex, whereas others occur very high in the clustergram so that they contain components that have low affinity among themselves. This difficulty is perhaps one of the biggest challenges in accurately determining complexes. We note, however, that in some cases (such as the SAGA complex described above), the correct complexes themselves (or a slight variant) were sometimes also members in our set of predictions, a situation possible because of the ability of HACO to make predictions at multiple levels of granularity. Thus, HACO is occasionally able to circumvent this challenge by trading off coverage for precision.

\section{A Comprehensive Interaction Network}

Complexes together with individual proteins comprise the basic units of the interaction network of the cell. So far, most of the work (27-29) has focused on predictions of interactions between pairs of individual proteins. However, the view of the network in terms of pairwise interactions loses much of its structure. Many interactions arise from co-complexness so that a single large complex can give rise to a very dense (almost complete) subgraph in the network. Other pairwise interactions are representatives of interactions between larger complexes. We therefore set out to construct a comprehensive network of interactions between all basic units in the proteome, both complexes and proteins.

We compiled a reference set of CCls and protein-complex interactions from reliable protein-protein interactions and 


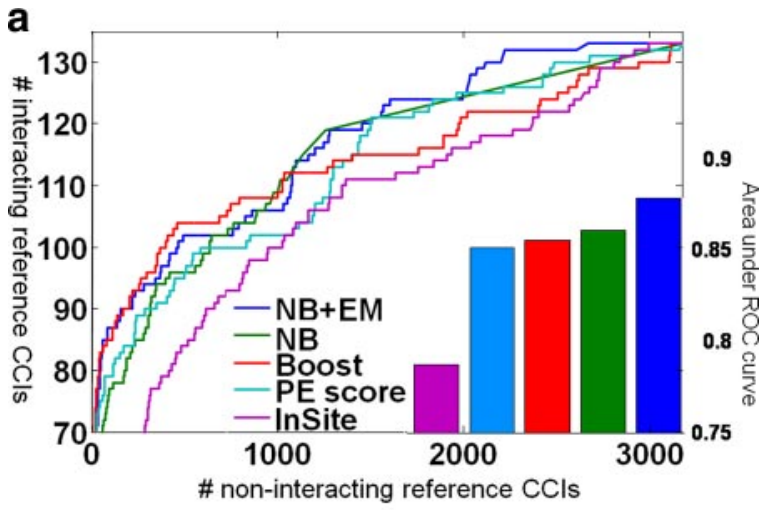

b

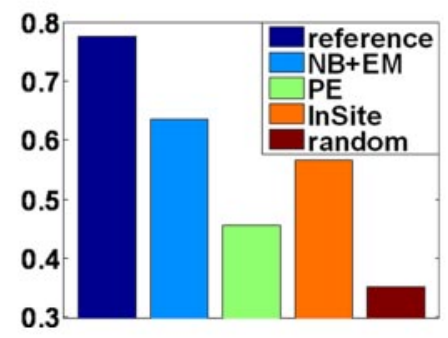

C

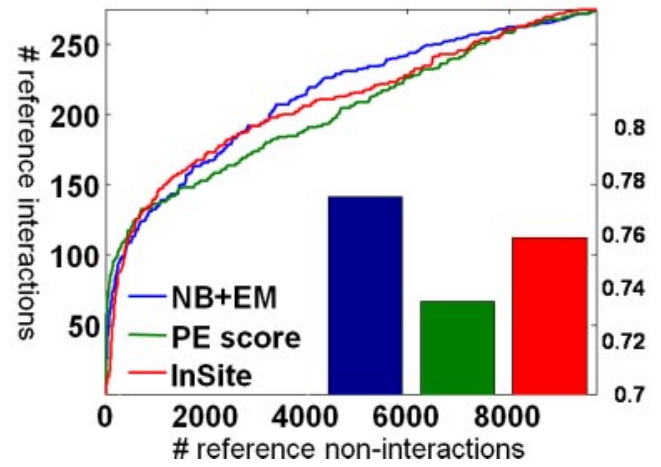

FIG. 5. Verification of complex-based interaction network. $a$, verification of our complex-complex interaction predictions relative to our reference set. Complex pairs in the hidden set of a 10-fold cross-validation are ranked based on their predicted interaction probabilities. Blue, green, and red curves are for the three models we tried. Light blue and pink curves are for the predictions using only PE score or InSite probabilities, respectively. Each point on the curve corresponds to a different threshold, giving rise to a different number of predicted interactions. The value on the $x$ axis is the number of pairs not in the reference set but predicted to interact. The value on the $y$ axis is the number of reference interactions that are predicted to interact. The bars in the right bottom corner are the areas under the ROC curves. Our naïve Bayes model with EM achieves the highest accuracy. The prediction made by PE score alone is slightly worse than our integrated models. $b$, functional coherence of interacting complexes measured by joint membership in the same MIPS functional category, a feature not used in training. We only consider those interacting complexes if both of them are assigned to some MIPS category. We picked the top 500 predictions from our NB + EM model and the top 500 obtained from the PE score alone. We compared them with complex pairs in our reference set and randomly selected pairs. The $y$ axis shows the proportion of interacting complexes that are assigned to the same MIPS category. As we can see, $59.2 \%$ of our predicted interacting complexes share the same MIPS category, whereas only 35.2 and $45.5 \%$ share the same category for hand-curation (see "Experimental Procedures"). Importantly to avoid circular reasoning, any interactions that we used in the construction of the gold standard CCls and protein-complex interactions were not given as features to the prediction algorithm. We used 10-fold cross-validation to evaluate the ability of our model in accurately predicting CCls. We randomly divide our reference interactions into 10 sets. In each fold, we hide one set and train on the remaining nine sets. We then make predictions on the held-out set using the learned model. We compare three methods (see "Experimental Procedures"): simple naïve Bayes, a discriminative boosting method, and naïve Bayes with EM (NB + EM) that also makes use of the data for pairs that are not in our reference set. As we can see in Fig. 5a, NB + EM performs better than both other methods, achieving very high performance: 44 of the top 50 predictions (88\%) are in the positive reference set. We also compared these results with two state-of-the-art methods for predicting protein-protein interactions: the PE score and the InSite probabilities. As we can see, by integrating multiple sources of data, we are able to improve the accuracy to 0.88 (area under the ROC curve) from 0.85 and 0.79 for PE score and InSite probabilities, respectively.

The PE score provides the strongest signal and provides, by itself, accuracy on our reference set that is only somewhat lower than that of our integrated model. However, when evaluated on other metrics, our data integration provides more significant benefits. We expect interacting complexes to be involved in similar biological processes. Therefore, we measured the coherence of our $\mathrm{CCl}$ predictions relative to $\mathrm{GO}$ biological process annotations and MIPS functional categories (neither of which were used in training). Here our predictions are considerably better than those obtained from the PE score alone (Fig. 5b), suggesting that our set of reference complexes is perhaps somewhat biased toward areas that are well covered by the TAP-MS assays.

We also apply our model to predict a unified network involving both proteins and complexes, a network that we call ComplexNet. In ComplexNet, we have both the interactions

random complex pairs and for those predicted by PE score alone, respectively. The reference complexes are the most coherent, a fact that is not surprising as the functional classification of reference complexes is sometimes derived from the same literature sources as the interactions between those complexes. $c$, verification against a reference set of our unified predictions of protein-complex and complex-complex interaction set. Complex pairs in the hidden set of a 10-fold cross-validation are ranked based on their predicted interaction probabilities. The blue curve is for our naïve Bayes model with EM. The light blue curve is for the predictions using only PE score. The pink curve is for the prediction using only InSite probability. Each point on the curve corresponds to a different threshold, giving rise to a different number of predicted interactions. The value on the $x$ axis is the number of pairs not in the reference set but predicted to interact. The value on the $y$ axis is the number of reference interactions that are predicted to interact. The areas under the curves are shown by the bars in the bottom right corner. 
between two complexes and the interactions between a protein and a complex. As we can see from Fig. $5 c$, by integrating multiple data sources, our naïve Bayes model with EM is able to achieve higher accuracy than using either PE score or InSite probability alone. We generated predictions for all protein-complex pairs and complex-complex pairs by training on the entire reference set (see our supporting Web site ${ }^{3}$ for the complete list of the predictions). Overall our predictions provide a comprehensive network of all of the interactions involving complexes. It can be combined with a set of high quality protein-protein interactions (such as Ref. 49) to provide a complete set of predictions for the $S$. cerevisiae protein interaction network. Fig. 6 presents a fragment of the network.

We identified many CCls that were expected and well characterized but not in the reference set, such as interactions between histones and several chromatin-modifying complexes including the ISW1 complex, the HAT1 complex, and RSC. ComplexNet also suggests novel hypotheses, several of which have support in the literature. For example, we predicted an interaction between complex 1035, which consists of the poorly characterized proteins Yer071c and Yir003w/ Aim21, with the yeast actin-capping protein (a Cap1-Cap2 heterodimer). Consistent with this prediction, high throughput fluorescence microscopy found that Yir003w co-localizes with components of the actin cytoskeleton (37), and two-hybrid data has connected Yir003w to the actin-binding protein Abp1 (79). Additionally like deletion of CAP1 or CAP2, deletion of YER071C or YIR003W results in strong sensitivity to the actin-depolymerizing agent latrunculin (45). Our observation suggests a more specific placement of this complex among the actin regulatory machinery. We also found several interesting interactions involving the centromere-localized kinetochore complex (Fig. 6), some of which have independent support. Our prediction of an interaction between the kinetochore and the proteasome is supported by a recent report that levels of Cse4, a centromere-localized histone, are regulated by ubiquitin-proteasome-mediated proteolysis (80). Our predicted link between the kinetochore and the spliceosome is consistent with evidence of a functional connection between these two factors (81). The remaining connections we observed with the kinetochore (pre-60 S ribosomal particle and RNA polymerase III) are intriguing, but more work will be required to determine the validity and functional significance of these predicted relationships. We can also learn from the false positive predictions of CCls. Our algorithm does make some apparently false positive predictions, and many of them fall into two main categories. Pairs of complexes that share a substantial number of common components, such as the SWR complex and NuA4, are sometimes identified as interacting. Additionally pairs of complexes that do not interact directly but are one link away in the interaction network are sometimes identified. Along these lines, we identified an interaction between the NuA4 histone acetylase complex and the opposing RPD3(L) deacetylase complex. Both complexes have subunits with specificity for binding Lys ${ }^{4}$ trimethylated histone $\mathrm{H} 3$ (82) and have been found to be regulated by binding to 14-3-3 proteins (83). Thus, even such a false positive may still provide interesting biological insights.

\section{Essentiality and Complex Size}

Much discussion has occurred regarding the relationship between essentiality and the structure of the protein-protein interaction network. Early work of Jeong et al. (26) and Han et al. (84) found that hub proteins in a protein-protein interaction network are more likely to be encoded by essential genes. More recent work (85) suggests that highly connected proteins are simply more likely to participate in essential proteinprotein interactions and are therefore more likely to be essential. However, a deeper insight on the relationship between the protein network and essentiality can be obtained by considering the network at the level of complexes rather than pairwise interactions. Such an analysis was recently performed by Hart et al. (5), who showed that essential proteins are concentrated in certain complexes, resulting in a dichotomy of essential and non-essential complexes. This phenomenon was also found in our predicted complexes (Fig. 7a). However, that finding does not explain why hubs in the network are more likely to be essential. We therefore looked into the distribution of essential proteins in complexes of different sizes and found that the fraction of essential components in a complex tends to increase with complex size (Fig. 7b). Moreover when we aggregate over all complexes of a given size, larger complexes tend to have a far greater proportion of essential proteins among their components (Fig. 7b). Components in a large complex are naturally highly connected in the protein interaction network and therefore often form hubs. Thus, the finding regarding the essentiality of hubs very likely arises from the fact that large complexes are more likely to have a much higher ratio of essential genes. Our finding is consistent with the recent work of Zotenko et al. (86), who argue that essential hubs are often members of a densely connected set of proteins performing an essential cellular function. However, this analysis is still performed on the pairwise protein network and hence is unable to identify the strong dependence between the size of a complex and its essentiality.

To test whether our finding truly explains the phenomenon of essential hubs, we tested whether essentiality is better explained by complex size or by hubness. We rank every protein based on the size of the largest complex to which it belongs and for the $K$ top-ranked proteins (for different values of $K$ ) plot the number of essential versus non-essential proteins (Fig. 7c). We plotted a similar curve by using the hubness of the protein, the degree in the yeast two-hybrid proteinprotein interaction network $(35,36)$. As we can see, complex size is a much better predictor for essentiality than hubness. We note that if we use the scaled PE score (at threshold $>0.5$ ) 
FIG. 6. A complex-level interaction network. a, a fragment of our ComplexNet, comprising a subset of the interactions between the largest complexes. Shown are the 500 highest confidence predictions plus the reference interactions restricted to interactions between complexes of size $\geq 3$. The color of each complex indicates the fraction of essential components, demonstrating the enrichment of essential proteins in larger complexes. The complexes are placed in regions based on their cellular localization determined by majority vote based on the data of Huh et al. (37). The inset shows all interactions that involve the kinetochore complex. $b$, a list of the complexes associated with the numbers in the figure. Complexes are associated with a name of a known complex when they overlap with that complex with Fscore $>0.5$. Otherwise the number associated with a complex is a unique identifier used in our supporting Web site. ${ }^{3}$ $E R$, endoplasmic reticulum. a

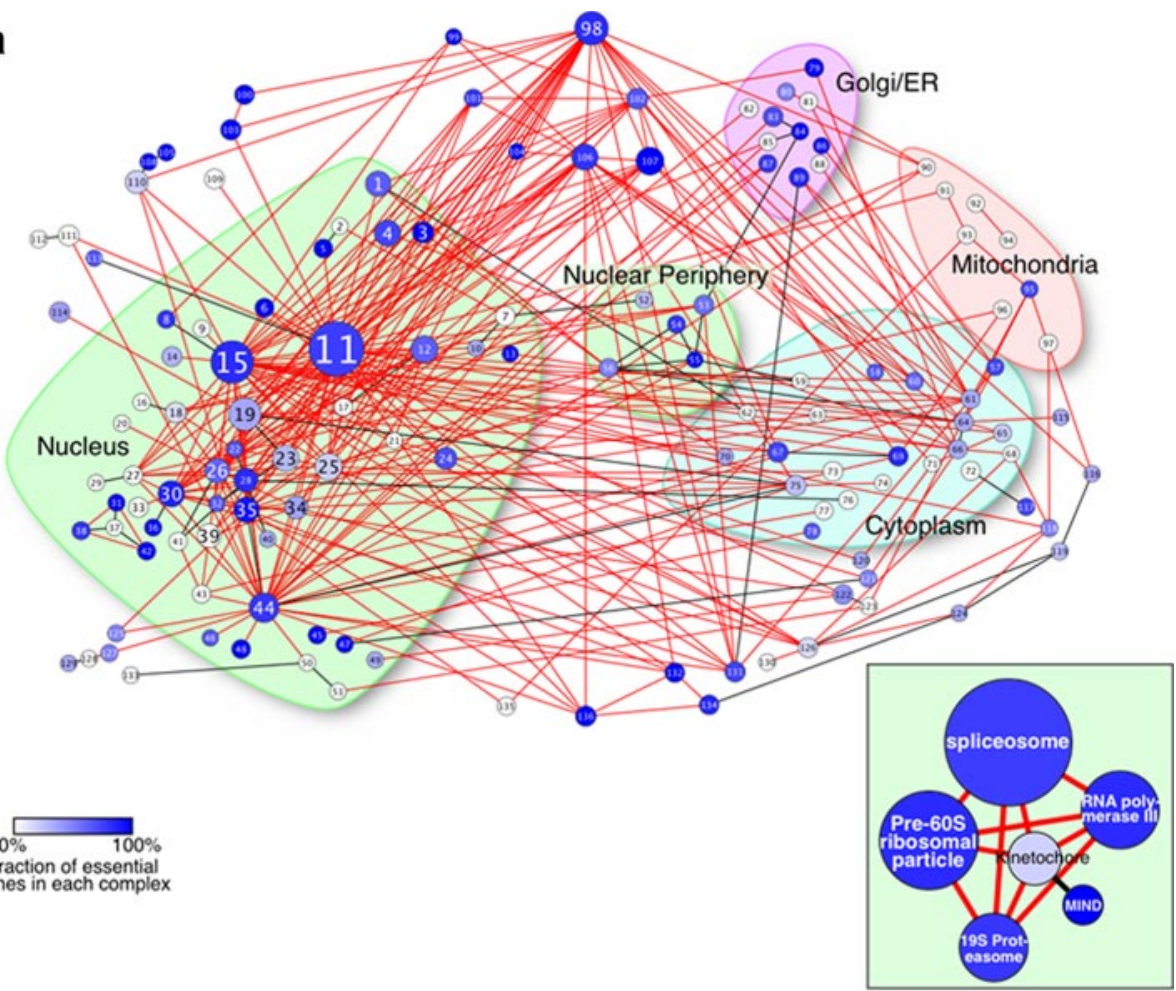

b
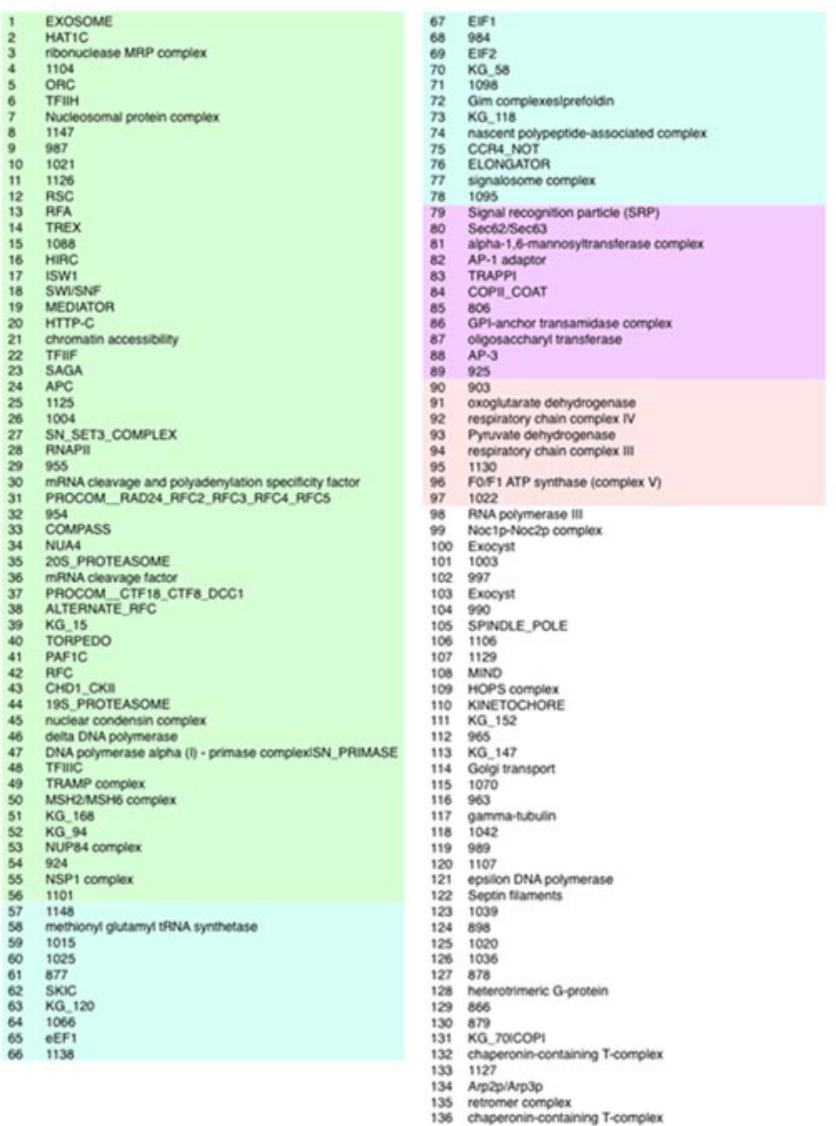


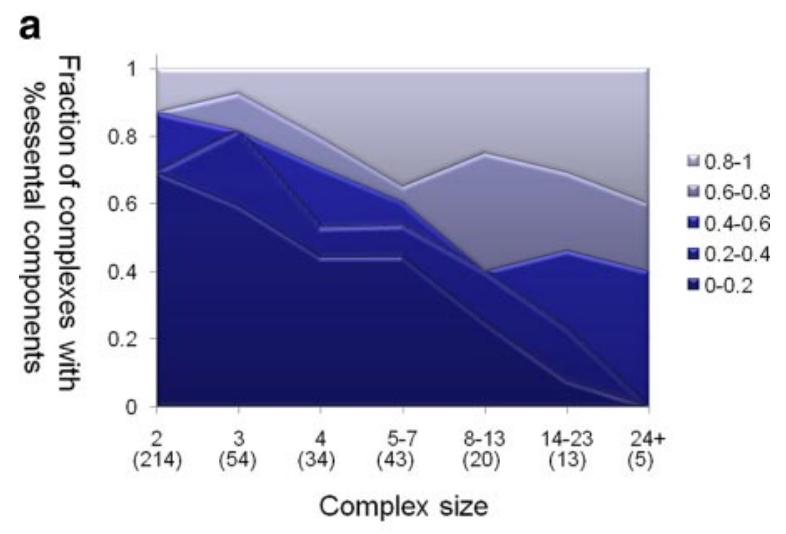

b

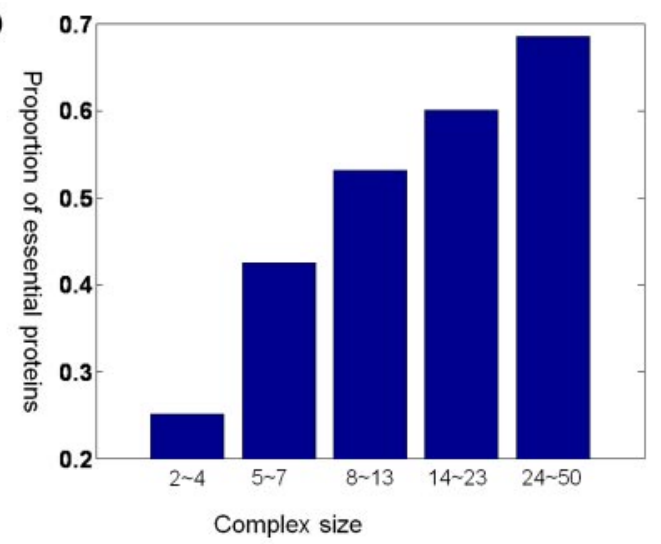

C

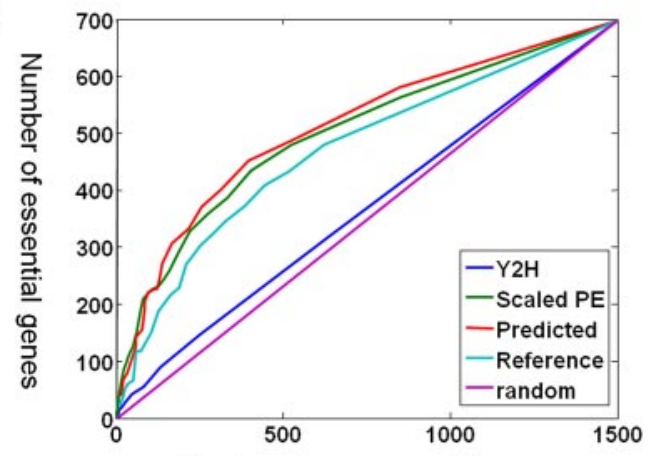

Number of non-essential genes

FIG. 7. Relationship between complex size and essentiality. a, fraction of complexes with different essentiality fractions. Each complex is represented by its size and the fraction of essential components. The different colors represent different ratios of essentiality in a complex discretized into five bins. The $x$ axis represents the complex size, and the $y$ axis represents the fraction of complexes of that size that have this particular essentiality ratio. We can see that the large majority of complexes of size 2 have essentiality ratio in the range $0-0.2$, whereas larger complexes tend to have a larger essentiality ratio. Also shown on the $x$ axis, in parentheses, is the number of complexes in each category (e.g. there are 54 complexes of size 3). $b$, the relationship between complex size and the proportion of essential proteins in complexes of that size. The $x$ axis is the size bin of the complexes. The $y$ axis is the proportion of essential proteins in all complexes within the size bin. As we can see, larger complexes tend to have a higher proportion of essential proteins. $c$, evaluation of different metrics as predictive of essentiality: size of the largest enclosing complex versus degree in the protein-protein interaction to define a protein-interaction network the hubness becomes a strong predictor of protein essentiality. However, PE score is more related to co-complexness than interaction, and thus this metric of hubness is directly related to complex size. Nevertheless using complex size directly is still better than using scaled PE score. Interestingly if we use the size of the largest enclosing reference complex to rank each protein, the result is slightly less predictive than using our predicted complexes or even the scaled PE score directly.

\section{DISCUSSION}

Identifying a comprehensive set of protein complexes in yeast is an important but challenging task. The high quality and high throughput TAP-MS data, which directly measure co-complexness, provide a starting point for accurately reconstructing these complexes. Indeed two recent studies (5, 6) used the TAP-MS data to produce a set of complexes with state-of-the-art performances. Both methods applied a simple clustering algorithm to a score derived directly from the TAP-MS data. In this study, we are able to significantly improve the accuracy of the complex reconstruction in three ways. First, we carefully constructed a large set of reference complexes and trained our model so it specifically predicts co-membership in stoichiometrically stable complexes. Second, we integrated multiple sources of heterogeneous data so our predictions are more robust to noise and incomplete coverage in the TAP-MS data. Finally we extended the highly effective HAC algorithm to allow reconstruction of clusters with overlap, a flexibility that allows it to circumvent many of the limitations of the standard HAC algorithm. We show that the resulting set of predicted complexes (available from our Web site $^{3}$ ) has significantly higher accuracy and is more biologically coherent than that of other recent methods. In many cases, it is even more coherent than the reference set, indicating it is of high quality and can be used as a new reference set. When combined with our comprehensive, hand-curated reference set (also available from our Web site $^{3}$ ), our work provides a significant new resource to the research community.

network (hubness). For the red and light blue curves, we rank each protein based on the size of the largest complex to which is belongs; the red curve uses predicted complexes, and the light blue curve uses the reference complexes. For the blue curve and green curve, we use the hubness, the degree of protein in a protein-protein interaction network; the blue curve uses the yeast two-hybrid protein-protein interaction network, and the green curve uses a network where pairs are connected if they have a scaled PE score $>0.5$. The $x$ axis is the number of essential proteins in the $K$ top ranked proteins (for different values of $K$ ), and the $y$ axis is the number of non-essential proteins. Complex size in our predicted complexes (red) is the best predictor for essentiality. The hubness based on PE score (green) performs better than the other metrics presumably because it also correlates directly with co-membership in a complex. The reference complexes (light blue) perform slightly worse but considerably better than interactions in the $\mathrm{Y} 2 \mathrm{H}$ data. 
With our high quality set of complexes, we are able to take a higher level perspective on the protein-protein interaction network, viewing it in terms of interactions between atomic units (whether individual proteins or stable complexes). There has been much work on predicting protein-protein interactions. However, these pairwise interactions are often induced by higher level relationships: those within a complex and those between complexes. Interactions within a complex give rise to densely connected subgraphs in the interaction network; interactions between complexes can give rise to a network of interconnections involving different members of the two complexes. Viewing the network in terms of its atomic units can help clarify its structure and its basic properties. We therefore defined the novel problem of predicting interactions between complexes and other complexes or proteins and constructed a new, high accuracy method for making such predictions. The result of our analysis is ComplexNet, a unified interaction network involving both proteins and complexes. We can now analyze the properties of this network, which better captures the true interactions underlying cellular processes. In particular, this network provides a new perspective on the previously observed relationship between the "hubness" of a protein in the network and its essentiality, demonstrating that larger complexes are more likely to be essential and comprise a large fraction of essential proteins. It would also be of interest to study other properties of this network, such as its connectivity or hierarchical structure.

To find a coherent set of proteins that form a complex, we have the choice of many different clustering algorithms. Brohee and van Helden (87) showed that MCL works well on a protein-protein interaction network by comparing it with three other clustering algorithms in the literature. So not surprisingly, Pu et al. (6) and Hart et al. (5) applied MCL to the TAP-MS network; MCL is confirmed by our results to be better than other existing methods in terms of reconstructing reference complexes and biological coherence. On the other hand, we found that HAC achieves about the same accuracy as MCL. Therefore, we focus on the best proven method and try to further improve it by addressing some of its limitations. One of the significant advantages of our HACO algorithm, which extends the HAC, is its ability to create overlapping complexes. Indeed the inability of traditional HAC to generate overlapping clusters is one of its major deficiencies in other types of data as well. Interestingly in our results, there were relatively few cases where two "correct" complexes shared subcomponents. Most of the benefit of HACO arose from avoiding mistakes arising from the greedy decisions of HAC and from allowing predictions at different levels of granularity (e.g. a complex and one of its subunits). Nevertheless the lack of extensive sharing of components between complexes was surprising given that such sharing is present in the reference set. To some extent, this phenomenon is due to the trade-off in HACO parameters between increasing the amount of component sharing and errors arising from merging of distinct complexes. However, HACO applied to other data sets (data not shown) did give rise to much more extensive sharing among different clusters. Thus, a complementary hypothesis is that some of the sharing of components between complexes arises when a protein plays roles in different complexes in different conditions. Our data, having been acquired almost entirely in YPD, would not reveal this condition-specific pleiotropy. It would be of great interest to acquire TAP-MS data in different conditions and study the extent to which complex structure is condition-specific.

We note that there are other clustering algorithms $(88,89)$ that also generate overlapping complexes. However, both of them are applicable only to a binary interaction network so an application to our task would require that we discretize the continuous affinities between protein pairs into two values (interacting and non-interacting) using some fixed threshold. Our analysis of the affinities for reference complexes suggested strongly that proteins that are co-complexed often exhibit affinities over a very broad range so that such a discretization would result in an unacceptable loss of useful information. On the other hand, HACO uses the continuous valued affinities directly, allowing the finer resolution of the computed affinities to be used by the algorithm. We also note that we devised several other novel methods that attempt to construct overlapping clusters. For example, one method directly learns an affinity function to predict the likelihood that a set of proteins forms a complex, aiming to take advantage of features involving more than two proteins. HACO significantly outperformed all of our other proposed methods, and so we omit details.

There are still many reference complexes that are not matched by our predicted complexes. Many of them fall into roughly two categories. In the first category, proteins in the reference complex have high affinities with each other and are grouped as a set during the HACO procedure. However, they are not selected in our predictions because they are not at the granularity where we cut our HACO cluster-lattice. They then become subsets or supersets of some predicted complexes. In fact, if we use all the sets generated during our HACO procedure as predicted complexes, 136 reference complexes would be perfectly predicted and 243 would be well matched by some predicted complexes in comparison with 95 perfect matches and 189 good matches in our current predictions. However, this approach would result in far too many predictions (3478), greatly reducing sensitivity. This fact highlights the limitations in defining a universal level of affinity at which one determines that a group of proteins forms a stable complex and suggests that a more flexible technique may be a useful direction for future work. In the second category, the proteins in the reference complex do not have high affinities with each other. This situation arises when the signal in the data is not sufficiently strong to indicate that two proteins are likely to interact. As most of our signal comes from the TAP-MS data, such "blind spots" can arise from limitations of 
this assay, such as complexes of low abundance or that are membrane-bound. In particular, we note that the TAP-MS data were all acquired in a single condition (rich media), and some complexes may simply not be present in the cell in that condition. Our inability to recover such complexes arises not from computational limitation, but from limitations in the data. New experimental assays are needed before these complexes can be reconstructed.

Like other previous approaches, our method was developed in the context of $S$. cerevisiae where we have the most data relevant to protein-protein interactions. Having a high quality set of predicted complexes is of significant value even in yeast as yeast provides an excellent model for many core biological processes. Moreover many key complexes are conserved from yeast to human, making our complex predictions valuable also to analysis of higher level organisms. Finally our method is general purpose and can easily be applied more broadly. Its ability to integrate multiple sets of diverse data makes it suitable for other organisms where we may not have the same type of data available as in yeast. With the increasing amount of high throughput protein-protein interaction data, both from TAP-MS (90) and other assays $(30,32)$, we should soon be able to provide a high quality reconstruction of protein complexes in other organisms, including human.

Our work takes a step toward a more hierarchical view of the protein-protein interaction network, moving up from individual proteins to complexes as the basic interacting units. The next level of the hierarchy is the pathways that comprise cellular pathways. Although the notion of a "pathway" is not as well defined, it would nevertheless be very useful to reconstruct pathways that are comprised of interacting complexes and proteins. This type of analysis will give us a unified perspective on the underlying hierarchical organization of the cell and provide significant insight.

Acknowledgment-We thank Maureen Hillenmeyer for useful discussions and for early access to the chemical genomics data.

* This work was supported by the National Science Foundation and the Defense Advanced Research Projects Agency under the program Cognitive Assistant that Learns and Organizes (to H. W., B. K., and D. K.), the Howard Hughes Medical Institute (to S. R. C. and K. M. S.), an Ernst Schering postdoctoral fellowship (to D. F.), and Sandler Family funding (to N. J. K.).

S The on-line version of this article (available at http://www. mcponline.org) contains supplemental material.

$\S$ Both authors contributed equally to this work.

$\S \S$ To whom correspondence may be addressed. Tel.: 415-4762980; Fax: 415-514-9736; E-mail: krogan@cmp.ucsf.edu.

१1 To whom correspondence may be addressed. Tel.: 650-7236598; Fax: 650-725-1449; E-mail: koller@cs.stanford.edu.

\section{REFERENCES}

1. Gavin, A. C., Bosche, M., Krause, R., Grandi, P., Marzioch, M., Bauer, A., Schultz, J., Rick, J. M., Michon, A. M., Cruciat, C. M., Remor, M., Hofert, C., Schelder, M., Brajenovic, M., Ruffner, H., Merino, A., Klein, K., Hudak, M., Dickson, D., Rudi, T., Gnau, V., Bauch, A., Bastuck, S., Huhse, B., Leutwein, C., Heurtier, M. A., Copley, R. R., Edelmann, A., Querfurth, E., Rybin, V., Drewes, G., Raida, M., Bouwmeester, T., Bork, P., Seraphin,
B., Kuster, B., Neubauer, G., and Superti-Furga, G. (2002) Functional organization of the yeast proteome by systematic analysis of protein complexes. Nature 415, 141-147

2. Ho, Y., Gruhler, A., Heilbut, A., Bader, G. D., Moore, L., Adams, S. L., Millar, A., Taylor, P., Bennett, K., Boutilier, K., Yang, L., Wolting, C., Donaldson, I., Schandorff, S., Shewnarane, J., Vo, M., Taggart, J., Goudreault, M., Muskat, B., Alfarano, C., Dewar, D., Lin, Z., Michalickova, K., Willems, A. R., Sassi, H., Nielsen, P. A., Rasmussen, K. J., Andersen, J. R., Johansen, L. E., Hansen, L. H., Jespersen, H., Podtelejnikov, A., Nielsen, E., Crawford, J., Poulsen, V., Sorensen, B. D., Matthiesen, J., Hendrickson, R. C., Gleeson, F., Pawson, T., Moran, M. F., Durocher, D., Mann, M., Hogue, C. W., Figeys, D., and Tyers, M. (2002) Systematic identification of protein complexes in Saccharomyces cerevisiae by mass spectrometry. Nature 415, 180-183

3. Gavin, A. C., Aloy, P., Grandi, P., Krause, R., Boesche, M., Marzioch, M., Rau, C., Jensen, L. J., Bastuck, S., Dumpelfeld, B., Edelmann, A., Heurtier, M. A., Hoffman, V., Hoefert, C., Klein, K., Hudak, M., Michon, A. M., Schelder, M., Schirle, M., Remor, M., Rudi, T., Hooper, S., Bauer, A., Bouwmeester, T., Casari, G., Drewes, G., Neubauer, G., Rick, J. M., Kuster, B., Bork, P., Russell, R. B., and Superti-Furga, G. (2006) Proteome survey reveals modularity of the yeast cell machinery. Nature $\mathbf{4 4 0}$, 631-636

4. Krogan, N. J., Cagney, G., Yu, H., Zhong, G., Guo, X., Ignatchenko, A., Li, J., Pu, S., Datta, N., Tikuisis, A. P., Punna, T., Peregrin-Alvarez, J. M., Shales, M., Zhang, X., Davey, M., Robinson, M. D., Paccanaro, A., Bray, J. E., Sheung, A., Beattie, B., Richards, D. P., Canadien, V., Lalev, A., Mena, F., Wong, P., Starostine, A., Canete, M. M., Vlasblom, J., Wu, S., Orsi, C., Collins, S. R., Chandran, S., Haw, R., Rilstone, J. J., Gandi, K., Thompson, N. J., Musso, G., St Onge, P., Ghanny, S., Lam, M. H., Butland, G., Altaf-UI, A. M., Kanaya, S., Shilatifard, A., O'Shea, E., Weissman, J. S., Ingles, C. J., Hughes, T. R., Parkinson, J., Gerstein, M., Wodak, S. J., Emili, A., and Greenblatt, J. F. (2006) Global landscape of protein complexes in the yeast Saccharomyces cerevisiae. Nature $\mathbf{4 4 0}$, 637-643

5. Hart, G. T., Lee, I., and Marcotte, E. R. (2007) A high-accuracy consensus map of yeast protein complexes reveals modular nature of gene essentiality. BMC Bioinformatics 8, 236

6. Pu, S., Vlasblom, J., Emili, A., Greenblatt, J., and Wodak, S. J. (2007) Identifying functional modules in the physical interactome of Saccharomyces cerevisiae. Proteomics 7, 944-960

7. Collins, S. R., Kemmeren, P., Zhao, X. C., Greenblatt, J. F., Spencer, F., Holstege, F. C., Weissman, J. S., and Krogan, N. J. (2007) Toward a comprehensive atlas of the physical interactome of Saccharomyces cerevisiae. Mol. Cell. Proteomics 6, 439-450

8. Mewes, H. W., Frishman, D., Mayer, K. F., Munsterkotter, M., Noubibou, O., Pagel, P., Rattei, T., Oesterheld, M., Ruepp, A., and Stumpflen, V. (2006) MIPS: analysis and annotation of proteins from whole genomes in 2005. Nucleic Acids Res. 34, D169-D172

9. Enright, A. J., Van Dongen, S., and Ouzounis, C. A. (2002) An efficient algorithm for large-scale detection of protein families. Nucleic Acids Res. 30, 1575-1584

10. Jansen, R., Yu, H., Greenbaum, D., Kluger, Y., Krogan, N. J., Chung, S., Emili, A., Snyder, M., Greenblatt, J. F., and Gerstein, M. (2003) A Bayesian networks approach for predicting protein-protein interactions from genomic data. Science 302, 449-453

11. Zhang, L. V., Wong, S. L., King, O. D., and Roth, F. P. (2004) Predicting co-complexed protein pairs using genomic and proteomic data integration. BMC Bioinformatics 5, 38

12. Qiu, J., and Noble, W. S. (2008) Predicting co-complexed protein pairs from heterogeneous data. PLoS Comput. Biol. 4, e1000054

13. Chen, J., and Yuan, B. (2006) Detecting functional modules in the yeast protein-protein interaction network. Bioinformatics 22, 2283-2290

14. Lee, I., Li, Z., and Marcotte, E. M. (2007) An improved, bias-reduced probabilistic functional gene network of baker's yeast, Saccharomyces cerevisiae. PLOS ONE 2, e988

15. Marcotte, E. M., Pellegrini, M., Thompson, M. J., Yeates, T. O., and Eisenberg, D. (1999) A combined algorithm for genome-wide prediction of protein function. Nature $\mathbf{4 0 2 , 8 3 - 8 6}$

16. Schlitt, T., Palin, K., Rung, J., Dietmann, S., Lappe, M., Ukkonen, E., and Brazma, A. (2003) From gene networks to gene function. Genome Res. 13, 2568-2576 
17. Strong, M., Mallick, P., Pellegrini, M., Thompson, M. J., and Eisenberg, D. (2003) Inference of protein function and protein linkages in Mycobacterium tuberculosis based on prokaryotic genome organization: a combined computational approach. Genome Biol. 4, R59

18. von Mering, C., Zdobnov, E. M., Tsoka, S., Ciccarelli, F. D., Pereira-Leal, J. B., Ouzounis, C. A., and Bork, P. (2003) Genome evolution reveals biochemical networks and functional modules. Proc. Natl. Acad. Sci. U. S. A. $100,15428-15433$

19. von Mering, C., Huynen, M., Jaeggi, D., Schmidt, S., Bork, P., and Snel, B. (2003) STRING: a database of predicted functional associations between proteins. Nucleic Acids Res. 31, 258-261

20. Yanai, I., and DeLisi, C. (2002) The society of genes: networks of functional links between genes from comparative genomics. Genome Biol. 3, research0064

21. Yellaboina, S., Goyal, K., and Mande, S. C. (2007) Inferring genome-wide functional linkages in $\mathrm{E}$. coli by combining improved genome context methods: comparison with high-throughput experimental data. Genome Res. 17, 527-535

22. Collins, M., Schapire, R., and Singer, Y. (2002) Logistic regression, AdaBoost and Bregman distances. Mach. Learn. 48, 253-285

23. Eisen, M. B., Spellman, P. T., Brown, P. O., and Botstein, D. (1998) Cluster analysis and display of genome-wide expression patterns. Proc. Natl. Acad. Sci. U. S. A. 95, 14863-14868

24. Collins, S. R., Miller, K. M., Maas, N. L., Roguev, A., Fillingham, J., Chu, C. S., Schuldiner, M., Gebbia, M., Recht, J., Shales, M., Ding, H., Xu, H., Han, J., Ingvarsdottir, K., Cheng, B., Andrews, B., Boone, C., Berger, S. L., Hieter, P., Zhang, Z., Brown, G. W., Ingles, C. J., Emili, A., Allis, C. D., Toczyski, D. P., Weissman, J. S., Greenblatt, J. F., and Krogan, N. J. (2007) Functional dissection of protein complexes involved in yeast chromosome biology using a genetic interaction map. Nature 446, 806-810

25. Walther, T. C., Brickner, J. H., Aguilar, P. S., Bernales, S., Pantoja, C., and Walter, P. (2006) Eisosomes mark static sites of endocytosis. Nature 439, 998-1003

26. Jeong, H., Mason, S. P., Barabasi, A. L., and Oltvai, Z. N. (2001) Lethality and centrality in protein networks. Nature $411,41-42$

27. Wei, N., and Deng, X. W. (2003) The COP9 signalosome. Annu. Rev. Cell Dev. Biol. 19, 261-286

28. Liu, Y., Liu, N., and Zhao, H. (2005) Inferring protein-protein interactions through high-throughput interaction data from diverse organisms. Bioinformatics 21, 3279-3285

29. Bock, J. R., and Gough, D. A. (2001) Predicting protein-protein interactions from primary structure. Bioinformatics 17, 455-460

30. Stelzl, U., Worm, U., Lalowski, M., Haenig, C., Brembeck, F. H., Goehler, H., Stroedicke, M., Zenkner, M., Schoenherr, A., Koeppen, S., Timm, J., Mintzlaff, S., Abraham, C., Bock, N., Kietzmann, S., Goedde, A., Toksoz, E., Droege, A., Krobitsch, S., Korn, B., Birchmeier, W., Lehrach, H., and Wanker, E. E. (2005) A human protein-protein interaction network: a resource for annotating the proteome. Cell 122, 957-968

31. Cherry, J. M., Adler, C., Ball, C., Chervitz, S. A., Dwight, S. S., Hester, E. T., Jia, Y., Juvik, G., Roe, T., Schroeder, M., Weng, S., and Botstein, D. (1998) SGD: Saccharomyces Genome Database. Nucleic Acids Res. 26, 73-79

32. Peri, S., Navarro, J. D., Amanchy, R., Kristiansen, T. Z., Jonnalagadda, C. K., Surendranath, V., Niranjan, V., Muthusamy, B., Gandhi, T. K., Gronborg, M., Ibarrola, N., Deshpande, N., Shanker, K., Shivashankar, H. N., Rashmi, B. P., Ramya, M. A., Zhao, Z., Chandrika, K. N., Padma, N., Harsha, H. C., Yatish, A. J., Kavitha, M. P., Menezes, M., Choudhury, D. R., Suresh, S., Ghosh, N., Saravana, R., Chandran, S., Krishna, S., Joy, M., Anand, S. K., Madavan, V., Joseph, A., Wong, G. W., Schiemann, W. P., Constantinescu, S. N., Huang, L., Khosravi-Far, R., Steen, H., Tewari, M., Ghaffari, S., Blobe, G. C., Dang, C. V., Garcia, J. G., Pevsner, J., Jensen, O. N., Roepstorff, P., Deshpande, K. S., Chinnaiyan, A. M., Hamosh, A., Chakravarti, A., and Pandey, A. (2003) Development of human protein reference database as an initial platform for approaching systems biology in humans. Genome Res. 13, 2363-2371

33. Ashburner, M., Ball, C. A., Blake, J. A., Botstein, D., Butler, H., Cherry, J. M., Davis, A. P., Dolinski, K., Dwight, S. S., Eppig, J. T., Harris, M. A., Hill, D. P., Issel-Tarver, L., Kasarskis, A., Lewis, S., Matese, J. C., Richardson, J. E., Ringwald, M., Rubin, G. M., and Sherlock, G. (2000) Gene ontology: tool for the unification of biology. The Gene Ontology Consortium. Nat.

\section{Genet. 25, 25-29}

34. Gollub, J., Ball, C. A., Binkley, G., Demeter, J., Finkelstein, D. B., Hebert, J. M., Hernandez-Boussard, T., Jin, H., Kaloper, M., Matese, J. C., Schroeder, M., Brown, P. O., Botstein, D., and Sherlock, G. (2003) The Stanford Microarray Database: data access and quality assessment tools. Nucleic Acids Res. 31, 94-96

35. Ito, T., Chiba, T., Ozawa, R., Yoshida, M., Hattori, M., and Sakaki, Y. (2001) A comprehensive two-hybrid analysis to explore the yeast protein interactome. Proc. Natl. Acad. Sci. U. S. A. 98, 4569-4574

36. Uetz, P., Giot, L., Cagney, G., Mansfield, T. A., Judson, R. S., Knight, J. R., Lockshon, D., Narayan, V., Srinivasan, M., Pochart, P., Qureshi-Emili, A., Li, Y., Godwin, B., Conover, D., Kalbfleisch, T., Vijayadamodar, G., Yang, M., Johnston, M., Fields, S., and Rothberg, J. M. (2000) A comprehensive analysis of protein-protein interactions in Saccharomyces cerevisiae. Nature 403, 623-627

37. Huh, W. K., Falvo, J. V., Gerke, L. C., Carroll, A. S., Howson, R. W., Weissman, J. S., and O'Shea, E. K. (2003) Global analysis of protein localization in budding yeast. Nature $425,686-691$

38. Lord, P. W., Stevens, R. D., Brass, A., and Goble, C. A. (2003) Investigating semantic similarity measures across the Gene Ontology: the relationship between sequence and annotation. Bioinformatics 19, 1275-1283

39. Xenarios, I., Salwinski, L., Duan, X. Q. J., Higney, P., Kim, S. M., and Eisenberg, D. (2002) DIP; the Database of Interacting Proteins: a research tool for studying cellular networks of protein interactions. Nucleic Acids Res. 30, 303-305

40. Vapnik, V. (1999) The Nature of Statistical Learning Theory, 2nd Ed., SpringerVerlag, New York

41. Freund, Y., and Schapire, R. (1997) A decision-theoretic generalization of on-line learning and an application to boosting. J. Comput. Syst. Sci. 55, 119-139

42. Sokal, R., and Michener, C. (1958) A statistical method for evaluating systematic relationships. Univ. Kans. Sci. Bull. 38, 1409-1438

43. Krause, R., von Mering, C., and Bork, P. (2003) A comprehensive set of protein complexes in yeast: mining large scale protein-protein interaction screens. Bioinformatics 19, 1901-1908

44. Ghaemmaghami, S., Huh, W. K., Bower, K., Howson, R. W., Belle, A., Dephoure, N., O'Shea, E. K., and Weissman, J. S. (2003) Global analysis of protein expression in yeast. Nature $425,737-741$

45. Hillenmeyer, M. E., Fung, E., Wildenhain, J., Pierce, S. E., Hoon, S., Lee, W., Proctor, M., St Onge, R. P., Tyers, M., Koller, D., Altman, R. B., Davis, R. W., Nislow, C., and Giaever, G. (2008) The chemical genomic portrait of yeast: uncovering a phenotype for all genes. Science 320, 362-365

46. Maclsaac, K. D., Wang, T., Gordon, D. B., Gifford, D. K., Stormo, G. D., and Fraenkel, E. (2006) An improved map of conserved regulatory sites for Saccharomyces cerevisiae. BMC Bioinformatics 7, 113

47. Harbison, C. T., Gordon, D. B., Lee, T. I., Rinaldi, N. J., Macisaac, K. D., Danford, T. W., Hannett, N. M., Tagne, J. B., Reynolds, D. B., Yoo, J., Jennings, E. G., Zeitlinger, J., Pokholok, D. K., Kellis, M., Rolfe, P. A., Takusagawa, K. T., Lander, E. S., Gifford, D. K., Fraenkel, E., and Young, R. A. (2004) Transcriptional regulatory code of a eukaryotic genome. Nature 431, 99-104

48. Date, S. V., and Marcotte, E. M. (2003) Discovery of uncharacterized cellular systems by genome-wide analysis of functional linkages. Nat. Biotechnol. 21, 1055-1062

49. Wang, H., Segal, E., Ben-Hur, A., Li, Q. R., Vidal, M., and Koller, D. (2007) InSite: a computational method for identifying protein-protein interaction binding sites on a proteome-wide scale. Genome Biol. 8, R192

50. Zakrzewska, A., Boorsma, A., Brul, S., Hellingwerf, K. J., and Klis, F. M. (2005) Transcriptional response of Saccharomyces cerevisiae to the plasma membrane-perturbing compound chitosan. Eukaryot. Cell 4, 703-715

51. Mercier, G., Berthault, N., Touleimat, N., Kepes, F., Fourel, G., Gilson, E., and Dutreix, M. (2005) A haploid-specific transcriptional response to irradiation in Saccharomyces cerevisiae. Nucleic Acids Res. 33, $6635-6643$

52. Causton, H. C., Ren, B., Koh, S. S., Harbison, C. T., Kanin, E., Jennings, E. G., Lee, T. I., True, H. L., Lander, E. S., and Young, R. A. (2001) Remodeling of yeast genome expression in response to environmental changes. Mol. Biol. Cell 12, 323-337

53. Lai, L. C., Kosorukoff, A. L., Burke, P. V., and Kwast, K. E. (2005) Dynamical remodeling of the transcriptome during short-term anaerobiosis in Sac- 
charomyces cerevisiae: differential response and role of Msn2 and/or Msn4 and other factors in galactose and glucose media. Mol. Cell. Biol. 25, 4075-4091

54. O'Rourke, S. M., and Herskowitz, I. (2002) A third osmosensing branch in Saccharomyces cerevisiae requires the Msb2 protein and functions in parallel with the Sho1 branch. Mol. Cell. Biol. 22, 4739-4749

55. Gasch, A. P., Spellman, P. T., Kao, C. M., Carmel-Harel, O., Eisen, M. B., Storz, G., Botstein, D., and Brown, P. O. (2000) Genomic expression programs in the response of yeast cells to environmental changes. Mol. Biol. Cell 11, 4241-4257

56. Gasch, A. P., Huang, M., Metzner, S., Botstein, D., Elledge, S. J., and Brown, P. O. (2001) Genomic expression responses to DNA-damaging agents and the regulatory role of the yeast ATR homolog Mec1p. Mol. Biol. Cell 12, 2987-3003

57. DeRisi, J. L., lyer, V. R., and Brown, P. O. (1997) Exploring the metabolic and genetic control of gene expression on a genomic scale. Science 278, $680-686$

58. Kitagawa, E., Akama, K., and Iwahashi, H. (2005) Effects of iodine on global gene expression in Saccharomyces cerevisiae. Biosci. Biotechnol. Biochem. 69, 2285-2293

59. Shevchenko, A., Wilm, M., Vorm, O., and Mann, M. (1996) Mass spectrometric sequencing of proteins silver-stained polyacrylamide gels. Anal. Chem. 68, 850-858

60. Collins, S. R., Schuldiner, M., Krogan, N. J., and Weissman, J. S. (2006) A strategy for extracting and analyzing large-scale quantitative epistatic interaction data. Genome Biol. 7, R63

61. Schuldiner, M., Collins, S. R., Thompson, N. J., Denic, V., Bhamidipati, A., Punna, T., Ihmels, J., Andrews, B., Boone, C., Greenblatt, J. F., Weissman, J. S., and Krogan, N. J. (2005) Exploration of the function and organization of the yeast early secretory pathway through an epistatic miniarray profile. Cell 123, 507-519

62. Bader, G. D., and Hogue, C. W. (2003) An automated method for finding molecular complexes in large protein interaction networks. BMC Bioinformatics 4, 2

63. Kim, M., Krogan, N. J., Vasiljeva, L., Rando, O. J., Nedea, E., Greenblatt, J. F., and Buratowski, S. (2004) The yeast Rat1 exonuclease promotes transcription termination by RNA polymerase II. Nature 432, 517-522

64. Maytal-Kivity, V., Piran, R., Pick, E., Hofmann, K., and Glickman, M. H. (2002) COP9 signalosome components play a role in the mating pheromone response of S. cerevisiae. EMBO Rep. 3, 1215-1221

65. van Hoof, A., Staples, R. R., Baker, R. E., and Parker, R. (2000) Function of the ski4p (Csl4p) and Ski7p proteins in $3^{\prime}$-to-5' degradation of mRNA. Mol. Cell. Biol. 20, 8230-8243

66. Luke, M. M., Della Seta, F., Di Como, C. J., Sugimoto, H., Kobayashi, R., and Arndt, K. T. (1996) The SAP, a new family of proteins, associate and function positively with the SIT4 phosphatase. Mol. Cell. Biol. 16, 2744-2755

67. Denic, V., Quan, E. M., and Weissman, J. S. (2006) A luminal surveillance complex that selects misfolded glycoproteins for ER-associated degradation. Cell 126, 349-359

68. Carvalho, P., Goder, V., and Rapoport, T. A. (2006) Distinct ubiquitin-ligase complexes define convergent pathways for the degradation of ER proteins. Cell 126, 361-373

69. Dragon, F., Gallagher, J. E., Compagnone-Post, P. A., Mitchell, B. M., Porwancher, K. A., Wehner, K. A., Wormsley, S., Settlage, R. E., Shabanowitz, J., Osheim, Y., Beyer, A. L., Hunt, D. F., and Baserga, S. J. (2002) A large nucleolar U3 ribonucleoprotein required for $18 \mathrm{~S}$ ribosomal RNA biogenesis. Nature 417, 967-970

70. Alic, N., Higgins, V. J., and Dawes, I. W. (2001) Identification of a Saccharomyces cerevisiae gene that is required for $\mathrm{G} 1$ arrest in response to the lipid oxidation product linoleic acid hydroperoxide. Mol. Biol. Cell 12, 1801-1810

71. Fiedler, D., Braberg, H., Mehta, M., Chechik, G., Cagney, G., Mukherjee, P., Silva, A. C., Shales, M., Collins, S. R., van Wageningen, S., Kemmeren, P., Holstege, F. C. P., Weissman, J. S., Christopher-Keogh, M., Koller, D., Shokat, K. M., and Krogan, N. J. (2009) Functional organization of the S. cerevisiae phosphorylation network. Cell 136, 952-963

72. Jorgensen, P., Rupes, I., Sharom, J. R., Schneper, L., Broach, J. R., and Tyers, M. (2004) A dynamic transcriptional network communicates growth potential to ribosome synthesis and critical cell size. Genes Dev. 18, 2491-2505
73. Urban, J., Soulard, A., Huber, A., Lippman, S., Mukhopadhyay, D., Deloche, O., Wanke, V., Anrather, D., Ammerer, G., Riezman, H., Broach, J. R., De Virgilio, C., Hall, M. N., and Loewith, R. (2007) Sch9 is a major target of TORC1 in Saccharomyces cerevisiae. Mol. Cell 26, 663-674

74. Garcia-Barrio, M., Dong, J., Ufano, S., and Hinnebusch, A. G. (2000) Association of GCN1-GCN20 regulatory complex with the N-terminus of elF2alpha kinase GCN2 is required for GCN2 activation. EMBO J. 19, 1887-1899

75. Powell, D. W., Weaver, C. M., Jennings, J. L., McAfee, K. J., He, Y., Weil, P. A., and Link, A. J. (2004) Cluster analysis of mass spectrometry data reveals a novel component of SAGA. Mol. Cell. Biol. 24, 7249-7259

76. Ingvarsdottir, K., Krogan, N. J., Emre, N. C., Wyce, A., Thompson, N. J., Emili, A., Hughes, T. R., Greenblatt, J. F., and Berger, S. L. (2005) H2B ubiquitin protease Ubp8 and Sgf11 constitute a discrete functional module within the Saccharomyces cerevisiae SAGA complex. Mol. Cell. Biol. 25, 1162-1172

77. Lee, K. K., Florens, L., Swanson, S. K., Washburn, M. P., and Workman, J. L. (2005) The deubiquitylation activity of Ubp8 is dependent upon Sgf11 and its association with the SAGA complex. Mol. Cell. Biol. 25, 1173-1182

78. Caruthers, J. M., and McKay, D. B. (2002) Helicase structure and mechanism. Curr. Opin. Struct. Biol. 12, 123-133

79. Fazi, B., Cope, M., Douangamath, A., Ferracuti, S., Schirwitz, K., Zucconi, A., Drubin, D., Wilmanns, M., Cesareni, G., and Castagnoli, L. (2002) Unusual binding properties of the $\mathrm{SH} 3$ domain of the yeast actin-binding protein Abp1: structural and functional analysis. J. Biol. Chem. 277, 5290-5298

80. Collins, K. A., Furuyama, S., and Biggins, S. (2004) Proteolysis contributes to the exclusive centromere localization of the yeast Cse4/CENP-A histone $\mathrm{H} 3$ variant. Curr. Biol. 14, 1968-1972

81. Bialkowska, A., and Kurlandzka, A. (2002) Proteins interacting with Lin 1p, a putative link between chromosome segregation, mRNA splicing and DNA replication in Saccharomyces cerevisiae. Yeast 19, 1323-1333

82. Shi, X., Kachirskaia, I., Walter, K. L., Kuo, J. H., Lake, A., Davrazou, F., Chan, S. M., Martin, D. G., Fingerman, I. M., Briggs, S. D., Howe, L., Utz, P. J., Kutateladze, T. G., Lugovskoy, A. A., Bedford, M. T., and Gozani, O. (2007) Proteome-wide analysis in Saccharomyces cerevisiae identifies several PHD fingers as novel direct and selective binding modules of histone $\mathrm{H} 3$ methylated at either lysine 4 or lysine 36. J. Biol. Chem. 282, 2450-2455

83. Lottersberger, F., Panza, A., Lucchini, G., and Longhese, M. P. (2007) Functional and physical interactions between yeast 14-3-3 proteins, acetyltransferases, and deacetylases in response to DNA replication perturbations. Mol. Cell. Biol. 27, 3266-3281

84. Han, J. D., Bertin, N., Hao, T., Goldberg, D. S., Berriz, G. F., Zhang, L. V., Dupuy, D., Walhout, A. J., Cusick, M. E., Roth, F. P., and Vidal, M. (2004) Evidence for dynamically organized modularity in the yeast proteinprotein interaction network. Nature 430, 88-93

85. He, X., and Zhang, J. (2006) Why do hubs tend to be essential in protein networks? PLoS Genet. 2, e88

86. Zotenko, E., Mestre, J., O'Leary, D. P., and Przytycka, T. M. (2008) Why do hubs in the yeast protein interaction network tend to be essential: reexamining the connection between the network topology and essentiality. PLoS Comput. Biol. 4, e1000140

87. Brohee, S., and van Helden, J. (2006) Evaluation of clustering algorithms for protein-protein interaction networks. BMC Bioinformatics 7, 488

88. Palla, G., Derenyi, I., Farkas, I., and Vicsek, T. (2005) Uncovering the overlapping community structure of complex networks in nature and society. Nature $435,814-818$

89. Yu, H., Paccanaro, A., Trifonov, V., and Gerstein, M. (2006) Predicting interactions in protein networks by completing defective cliques. Bioinformatics 22, 823-829

90. Ewing, R. M., Chu, P., Elisma, F., Li, H., Taylor, P., Climie, S., McBroomCerajewski, L., Robinson, M. D., O'Connor, L., Li, M., Taylor, R., Dharsee, M., Ho, Y., Heilbut, A., Moore, L., Zhang, S., Ornatsky, O., Bukhman, Y. V., Ethier, M., Sheng, Y., Vasilescu, J., Abu-Farha, M., Lambert, J. P., Duewel, H. S., Stewart, I. I., Kuehl, B., Hogue, K., Colwill, K., Gladwish, K., Muskat, B., Kinach, R., Adams, S. L., Moran, M. F., Morin, G. B., Topaloglou, T., and Figeys, D. (2007) Large-scale mapping of human protein-protein interactions by mass spectrometry. Mol. Syst. Biol. 3, 89 



\section{nature \\ structural \& molecular biology}

\section{Eisosome-driven plasma membrane organization is mediated by BAR domains}

\author{
Natasza E Ziółkowska ${ }^{1}$, Lena Karotki ${ }^{1}$, Michael Rehman ${ }^{1}$, \\ Juha T Huiskonen $^{2} \&$ Tobias C Walther ${ }^{3}$
}

\begin{abstract}
Plasma membranes are organized into domains of different protein and lipid composition. Eisosomes are key complexes for yeast plasma membrane organization, containing primarily Pil1 and Lsp1. Here we show that both proteins consist mostly of a banana-shaped BAR domain common to membrane sculpting proteins, most similar to the ones of amphiphysin, arfaptin 2 and endophilin 2. Our data reveal a previously unrecognized family of BAR-domain proteins involved in plasma membrane organization.
\end{abstract}

Biological membranes are highly compartmentalized in time and space to segregate the many different biochemical reactions occurring there ${ }^{1}$. In many instances, organization of proteins and lipids into specialized membrane regions allows their efficient interactions, which in turn facilitates the flow of information and material between membranebound organelles or cells ${ }^{2}$. A fundamental question in cell biology is how plasma membrane organization is achieved molecularly.

Yeast provide an excellent model system to address this question since they features prominent patterning of the plasma membrane into compartments of distinct lipid and protein composition. Eisosomes are essential for formation of the membrane compartment containing Can1 (MCC) $)^{3-6}$. The main eisosome components in Saccharomyces cerevisiae are the abundant proteins Pill and Lsp1. To understand the mechanism of plasma membrane organization by eisosomes, we determined the molecular structure of eisosome components.

Limited proteolysis of full-length recombinant Lsp1 led to identification of a stable core fragment including residues 36 to 267, which we refer to as 'Lsp1 ASIA', denoting the start of its sequence (Ala-Ser-Ile-Ala).

Figure 1 Molecular structure of Lsp1 and Pil1. (a) X-ray structure of Lsp1 ASIA in a ribbon representation of the dimer (green and gray monomers). (b) Lsp1 sequence diagram showing the residues included in Lsp1 ASIA (green and yellow) and the residues not visible in the electron density map (yellow). (c) Structure alignment of A, B and C chains in the asymmetric unit of the Lsp1 ASIA X-ray structure. The gray portion was used to align the chains. The flexible parts of the structure are shown in blue, green and red (chains A, B and C). (d) Theoretical model of a Pill homodimer. Amino acids of low sequence similarity to Lspl are indicated in orange.
We solved the structure of Lsp1 ASIA by multiple-wavelength anomalous diffraction with the HKL3000 software package (ref. 7) at 2.9 Å resolution (Supplementary Table 1 and Supplementary Methods). A molecule of Lsp1 ASIA is composed of four antiparallel $\alpha$-helices that are connected by three loops. Three helices form a long, kinked coiled-coil (Fig. 1a). Lsp1 ASIA amino acids before residue 51 and after residue 267 (using the numbering for the full-length Lsp1) were not visible in the electron density maps (Fig. 1b). Two Lsp1 ASIA molecules form a banana-shaped dimer stabilized mainly by hydrophobic interactions. The asymmetric unit consists of three chains (A, B and C). Comparison of the three twofold symmetric dimers (AA, BB, CC), constructed by superposition of the three chains on the crystallographic dimer $A B$, revealed variation within the tips of the Lsp1 structure (Fig. 1c), indicating that this part of the protein is flexible.

Lsp1 and Pil1 show 72\% sequence identity (Supplementary Fig. 1), which facilitated homology modeling of the Pill homodimer when attempts to crystallize Pill or fragments from it were unsuccessful (Fig. 1d). Lsp1 and Pill are structurally most similar to BAR domains from Drosophila melanogaster amphiphysin (PDB 1URU) (Fig. 2a) ${ }^{8}$ and Homo sapiens arfaptin 2 (PDB $1 \mathrm{I} 49)^{9}$, which can be superimposed on Lsp1 ASIA with a root mean square difference of $3.3 \AA$ and $3.2 \AA$, respectively. Nonetheless, Lsp1 and other BAR domains have no strictly conserved pattern of amino acids (Supplementary Fig. 2). Sequence comparison and phylogenetic analysis of Lsp1 and Pil1 based on the structural alignment show a pattern of distant sequence

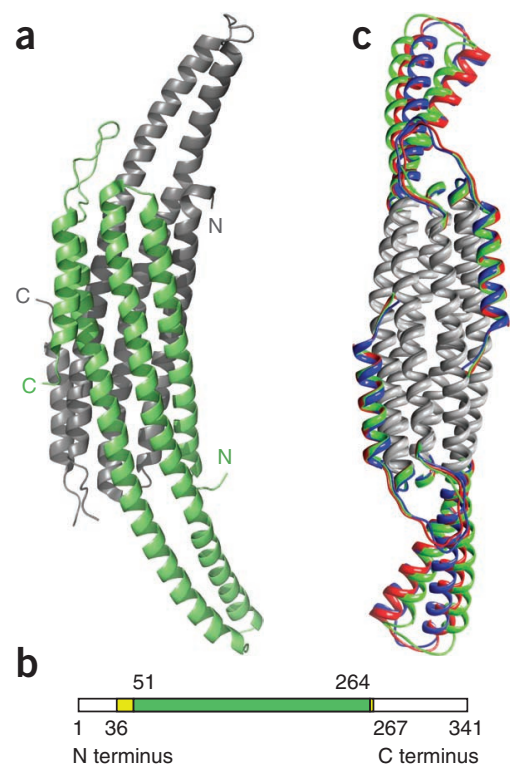

${ }^{1}$ Max Planck Institute of Biochemistry, Organelle Architecture and Dynamics, Martinsried, Germany. ${ }^{2}$ Oxford Particle Imaging Centre, Division of Structural Biology, Wellcome Trust Centre for Human Genetics, University of Oxford, Oxford, UK. ${ }^{3}$ Yale University School of Medicine, Department of Cell Biology, New Haven, Connecticut, USA. Correspondence should be addressed to T.C.W. (tobias.walther@yale.edu). 

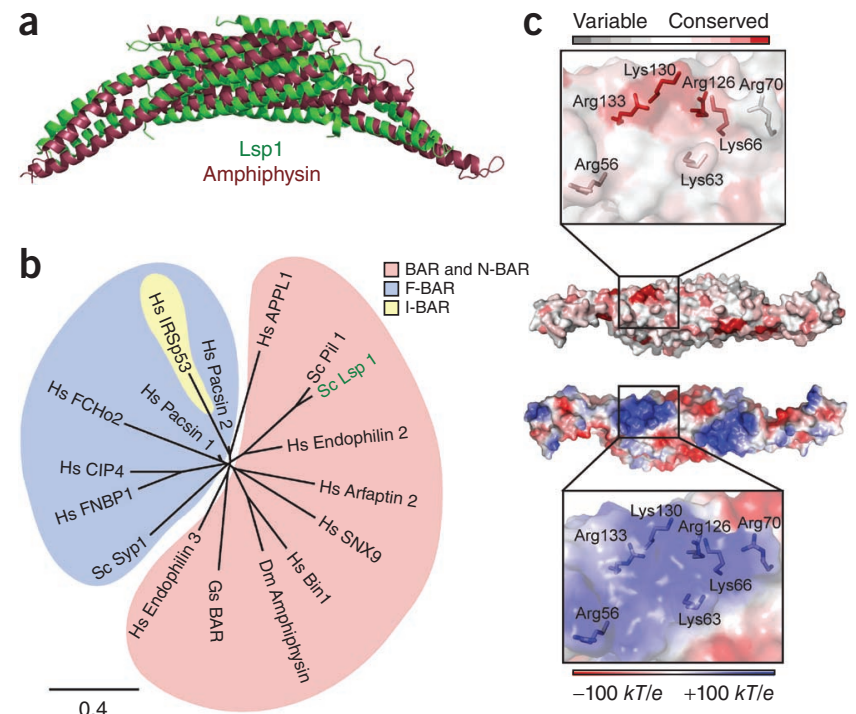

similarities, with the highest similarity to Lsp1 to human endophilin 2 (Fig. 2b). Together our structure and sequence comparison also show that the main eisosome proteins are closely related to classical BAR or N-BAR proteins, but are distinguished from F-BAR and I-BAR domains (Fig. 2b). Pill amino acid sequence alignments combined with secondary structure predictions identified a few hundred proteins with similarity to Pill and Lsp1, mostly in fungi, that we predict to contain BAR domains (Supplementary Fig. 3). BAR domains bind membranes to bend them or act as curvature sensing modules ${ }^{10}$. In agreement with this notion, we recently found that Pill and Lsp1 bind phosphatidylinositol-(4,5)-bisphosphate-containing membranes, a property retained by Lsp1 ASIA (unpublished data).

To investigate how Lsp1 and Pil1 may interact with membranes, we plotted the electrostatic potential of residues onto the Lsp1 ASIA surface, which revealed a patch with a strong positive charge on the concave surface of each monomer that is conserved in sequence among fungi (Fig. 2c). This positively charged surface patch of Pil1 and Lsp1 might mediate interaction with the membrane by binding to negatively charged lipid head groups-for example, of phosphatidylinositol- $(4,5)$ bisphosphate. We tested this hypothesis by expressing Pil1-GFP fusion proteins containing mutations in positive-patch residues in pill $1 \Delta$ or pill 1 lsp $1 \Delta$ cells and subsequently analyzing protein localization by confocal fluorescence microscopy (Fig. 3a-d, Supplementary Fig. 4 and Supplementary Methods). Many of the positive-patch mutations resulted in a strong effect on Pil1-GFP localization, mostly fewer eisosomes and more cytoplasmic Pill-GFP. Of particular note, expression of Pil1 R126E-GFP in pil1 $\Delta l s p 1 \Delta$ cells resulted in very long rods traversing

Figure 3 The positively charged patch on the Pill concave surface is required for normal Pil1 localization and function. (a) Representative confocal midsections of cells expressing Pil1-GFP wild-type (WT) and Pil1-GFP mutants (K130E R133E, R133E or K63A K66A K130A R133A (KKKR-A)) in pill $1 \Delta$ and pil $1 \Delta / s p 1 \Delta$ cells. Scale bar, $2.5 \mu \mathrm{m}$. (b) Quantification of foci per cell, normalized to WT. (c) Quantification of GFP fluorescence of foci. (d) Quantification of cytosolic GFP fluorescence. (e) Yeast strains with plasmids harboring a WT copy of PIL1 on a URA3 plasmid and a WT or mutant copy of $P I L 1$ on a LEU2 plasmid were tested for growth on 5-fluoroorotic acid (5-FOA) plates at $24^{\circ} \mathrm{C}$, which indicates the ability of the mutant copy of PILI to complement pil1 1 function. An agar plate with synthetic complete medium lacking uracil (- URA) is shown as a control for growth. pill mutants are indicated with pil1*. The yeast strains used in this work are listed in Supplementary Table 2. Error bars, s.d.

Figure 2 Lsp1 and Pil1 belong to the superfamily of BAR domaincontaining proteins. (a) Alignment of $S$. cerevisiae Lsp1 ASIA dimer (PDB 3PLT; green) and the D. melanogaster amphiphysin dimer (PDB IURU; brown). (b) Phylogenetic tree generated using a structure-based sequence alignment of Lsp1 ASIA with BAR domain-containing proteins. Dm, D. melanogaster; Hs. H. sapiens; Sc, S. cerevisiae; Gs, Galdieria sulphuraria. Scale bar in amino acid substitutions per site. (c) Lsp1 ASIA dimer surface colored according to evolutionary conservation (top panels) and electrostatic potential (bottom panels) on the concave surface and a close-up view of the positively charged surface patch.

the cytoplasm (Supplementary Fig. 5 and Supplementary Video 1). These phenotypes were not an effect of lack of Pill expression, because mutant proteins were expressed at the same abundance as wild-type protein (Supplementary Fig. 6). The effect of Pill mutants was generally dominant over the wild-type; for example, Pil1 K130E R133E-GFP expression led to relocalization of Pill-RFPmars into clusters at the membrane and into the cytosol (Supplementary Fig. 7). This finding suggests that Pill self-interacts and that a wild-type protein majority is required for normal eisosomes. To determine whether the positive surface patch of Pill is also required for normal plasma membrane organization, we expressed GFP-tagged mutants as the sole copy of Pil 1 in cells with the MCC marker protein Sur7 tagged with the red fluorescent protein RFPmars (Supplementary Fig. 8 and Supplementary Methods). Presence of either Pil1 K130E R133E or Pill KKKR-A (Pil1 K63A K66A K130A R133A) mutants led to the loss of the normal, punctuate distribution of Sur7-RFPmars along the plasma membrane and instead resulted in clusters of Sur7-RFPmars that colocalized with

a
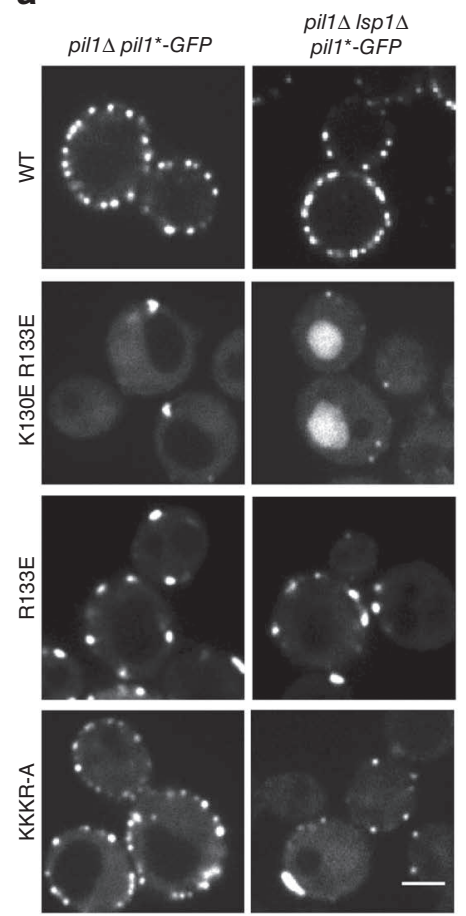

e
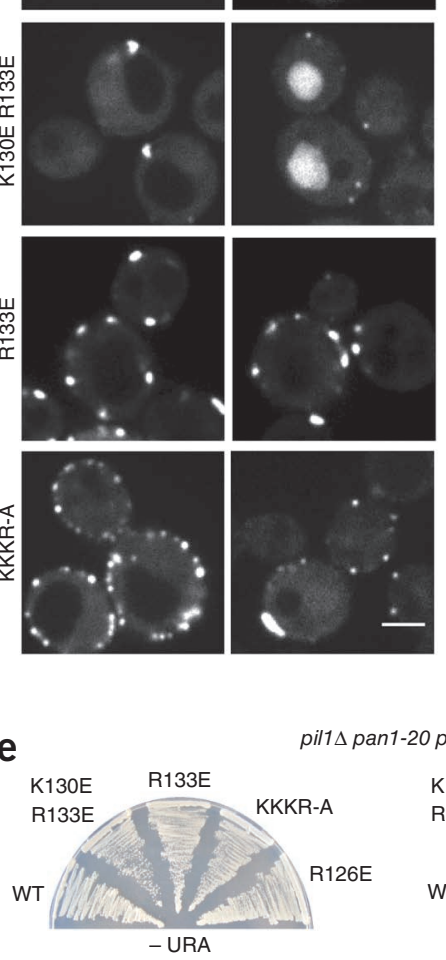

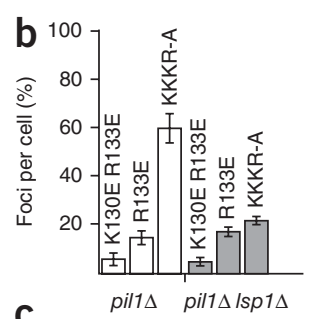

\section{c}

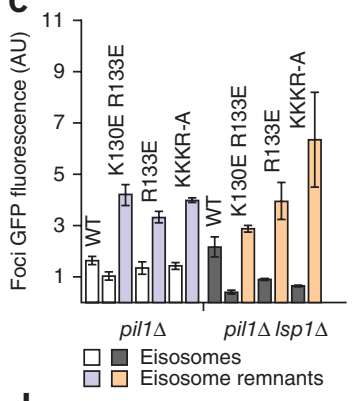

d
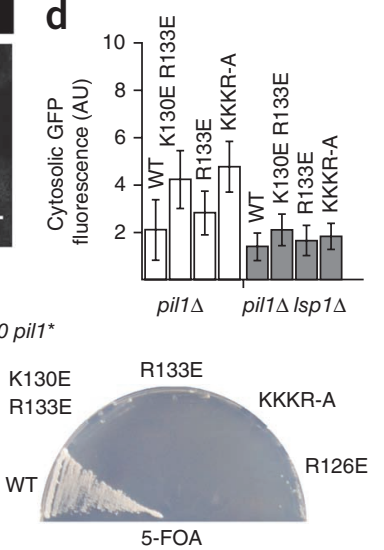
Pill-GFP mutants, thus showing that the positive patch is required for normal plasma membrane organization.

To further test the functionality of positive-patch mutants, we exploited the synthetic lethality between pil1 $\Delta$ and pan1-20, a hypomorphic allele of an endocytic adaptor protein associating with actin ${ }^{3}$ (Supplementary Methods). As expected, expression of wild-type Pil1 efficiently restored viability of pil1 $\Delta$ pan 1-20 cells. In contrast, expression of mutant proteins, such as pil1 K130E R133E, pil1 R133E, pil1 KKKR-A or pill R126E, could not rescue lethality (Fig. 3e). Together, these data show that the BAR domain of Pil1, and more specifically its positive patch, are required for Pill function.

Our data directly address a main question in eisosome biology by revealing that the main eisosome components are part of the evolutionarily conserved BAR domain protein family, members of which bind and mold membranes. This discovery further underlines the ancient origin and importance of this domain for membrane molding. It also shows that eisosomes are an excellent model to study plasma membrane organization by such domains in the experimentally easily amenable yeast system. The available data suggest that eisosome BAR domain components interact with the plasma membrane to generate a specific membrane environment that selects for proteins and lipids.

Accession codes. Protein Data Bank: Coordinates for Lsp1 ASIA has been deposited under accession code 3PLT.

Note: Supplementary information is available on the Nature Structural \& Molecular Biology website.

\section{ACKNOWLEDGMENTS}

We would like to thank P. de Camilli, E. Conti, F. Förster, T. Keil, W. Minor, S. Schuck, S. Suppmann, A. Wlodawer and the MPI-B Crystallization Facility for discussion and help with experiments and the German Research Foundation (N.E.Z. and T.C.W.), Academy of Finland (grant 130750, J.T.H.) and Boehringer Ingelheim fellowships (L.K.) for funding.

\section{AUTHOR CONTRIBUTIONS}

All authors contributed to design and execution of experiments. N.E.Z. produced the protein, grew crystals, solved the structure of Lsp1 and performed the confocal microscopy. N.E.Z. and T.C.W. wrote the manuscript.

\section{COMPETING FINANCIAL INTERESTS}

The authors declare no competing financial interests.

Published online at http://www.nature.com/nsmb/.

Reprints and permissions information is available online at http://www.nature.com/ reprints/index.html.

1. Simons, K. \& Ikonen, E. Nature 387, 569-572 (1997).

2. Lingwood, D. \& Simons, K. Science $327,46-50$ (2010).

3. Walther, T.C. et al. Nature 439, 998-1003 (2006).

4. Grossmann, G., Opekarova, M., Malinsky, J., Weig-Meckl, I. \& Tanner, W. EMBO J. 26, 1-8 (2007).

5. Strádalová, V. et al. J. Cell Sci. 122, 2887-2894 (2009).

6. Fröhlich, F. et al. J. Cell Biol. 185, 1227-1242 (2009).

7. Minor, W., Cymborowski, M., Otwinowski, Z. \& Chruszcz, M. Acta Crystallogr. D Biol. Crystallogr. 62, 859-866 (2006).

8. Peter, B.J. et al. Science 303, 495-499 (2004).

9. Tarricone, C. et al. Nature 411, 215-219 (2001).

10. Masuda, M. \& Mochizuki, N. Semin. Cell Dev. Biol. 21, 391-398 (2010). 


\section{Online Supplemental Material}

Eisosome-driven plasma membrane organization is mediated by BAR domains

Natasza E. Ziółkowska, Lena Karotki, Michael Rehman, Juha T. Huiskonen

and Tobias C. Walther 


\begin{abstract}
Lsp1 MHRTYSLRNQRAPTAAELQAPPPPPSSTKSKFFGKASIASSFRKNAAGNFGPELARKLSQ
Pil1 MHRTYSLRNSRAPTASQLQNPPPPPSTTKGRFFGKGGLAYSFRRSAAGAFGPELSRKLSQ

Lsp1 LVKTEKGVLRAMEVVASERREAAKQLSLWGADNDDDVSDVTDKLGVLIYELGELQDQFID

Pil1 LVKIEKNVLRSMELTANERRDAAKQLSIWGLENDDDVSD ITDKLGVLIYEVSELDDQFID

Lsp1 KYDQYRVTLKSIRNIEASVQPSRDRKEKITDEIAHLKYKDPQSTKIPVLEQELVRAEAES

Pil1 RYDQYRLTLKSIRDIEGSVQPSRDRKDKITDKIAYLKYKDPQSPKIEVLEQELVRAEAES

Lsp1 LVAEAQLSNITREKLKAAYSYMFDSLRELSEKFALIAGYGKALLELLDDSPVTPGEARPA

Pil1 LVAEAQLSNITRSKLRAAFNYQFDSIIEHSEKIALIAGYGKALLELLDDSPVTPGETRPA

Lsp1 YDGYEASRQIIMDAESALESWTLDMAAVKPTLSFHQTVDDVYEDEDGEEEEEPEIONGDI

Pil1 YDGYEASKQIIIDAESALNEWTLDSAQVKPTLSFKQDYEDFEPEEGEEEEEEDGQGRWSE

Lsp1 PGQVVEEEEVEWTTEVPVDDEAHEADHHVSQNGHTSGSENI

Pil1 DEQEDGQIEEPEQEEEGAVEEHEQVGHQQSESLPQQTTA--
\end{abstract}

Supplementary Fig.1.

Lsp1 and Pil1 exhibit high sequence similarity.

Sequence alignment of Lsp1 and Pil1. 
HELIX 1

$\begin{array}{ll} & * \\ \text { Lsp1 } & \text { GPELARKLSELVKTEKGVLRAMEVVASERREAAKQLSLWGAD } \\ \text { Amphiphysin } & \text {-NRQQASANRLQKEFNNYIRCVRAAQAASKTLMDSVCEIYE- } \\ \text { Arfaptin } & \text {-ELQIELLRETKRKYESVLQLGRALTAHLYSLIQTQHALGDA } \\ \text { Endophilin } & \text {-DVTNKVVAEILSKTTEYLQYPQTEGLIGDCMLKYGKELGE- } \\ \text { SNX9 } & \text { FTKADDGVKELLTVGQEHWKRCTPLPKEYQKIGKALQSLATV } \\ \text { APPL1 } & \text {-TAISNYMNQLYQAMHRIYDAQNELSAATHLTSKLLKEYEKQ } \\ \text { FCHO2 } & \text {-KHGQISTKELADFVRERATIEEAYSRSMTKLAKSASNY-SQ } \\ \text { IRSp53 } & \text {-NPSLRNFIAMGKNYEKALAGVTYAAKGYFDALVKMGELASE }\end{array}$

HELIX 2

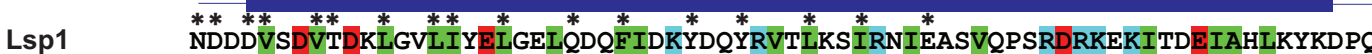
Amphiphysin PQWS---GYDALQAQTGASESLWADFAHKLGQVLIPLNTYTGQFPEMKKKVEKRNRKLIDYDGQRHSF-G Arfaptin FFGYNAETQKLLCKNGETLIGAVNFFVSSINTLVKTMEDTLMTVKQYEAARLEYDAYRTDLEELS-LGPR Endophilin ---DSTFGNALIEVGESMKLMAEVKDSLDINVKQFIDPLQLLQDKDKEIGHHLKKLEGRRLDYDYKK-E SNX9 FETDLNDAITEAGKTYEEIASLVAEQPKDLHFLMECNHEYKGFLGCFPDIIGTHKGAIEKVKESDKLVIT APPL1 -DEVMSSTLQQFSKVIDELSSCHAVLSTQLADMMFPITQFKEDLKEILTLKEVFQIASNDHDAAI-NRYS FCHO2 LGTFA-PVWDVFKTSTEKLANCHLDLVRKLQELIKEVQKYGEEQVSHKKTKVAGTLEAVQTIQSI-TQAL IRSP53 SQG-SKELGDVLFQMAEVHRQIQNQLEEMLKSFHNELLQLEQKVELDSYLSAALKKYQTEQRSKG-DALD

HELIX 3

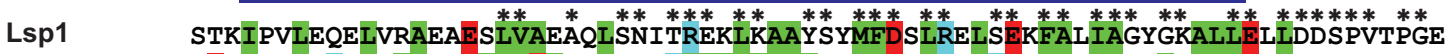
Amphiphysin REQLEEARRTYEILNTELHDELPALYDSRILFLVTNLQTLFATEQVFHNETAKIYSELEAIVDKLESQR----Arfaptin RGRLESAQATFQAHRDKYEKLRGDVAIKLKFLEENKIKVMHKQLILFHNAVSAYFAGNQKQLEQT--------Endophilin VRQAVEKFEESKELAERSMFNFLENDVEQVSQLAVFIEAALDYHRQSTEILQELQSKLQMRISAASVPR SNX9 LQDKQNMVKRVSIMSYALQAEMNHFHSNRIYDYNSVIRLYLEQQVQFYETIAEKIRQALSR--FPVM------APPL1 TEDVYTSRKKQHQTMMHYFCALNTLQYKKKIALLEPLLGYMQAQISFFKMGSENLNQLEEFLANISVQNEMDSD FCHO2 VEKYALAKADFEQKMTETAQKFQDIEETHLIHIKEI IGSLSNAIKEIHLQIGQVHEEF INNMANTTVE-----IRSP53 -NKQGELENYVSDGYKTALTEERRRFCFLVEKQCAVAKNSAAYHSKGKELLAQKLPLWQQACADPSKI-VQLMQ

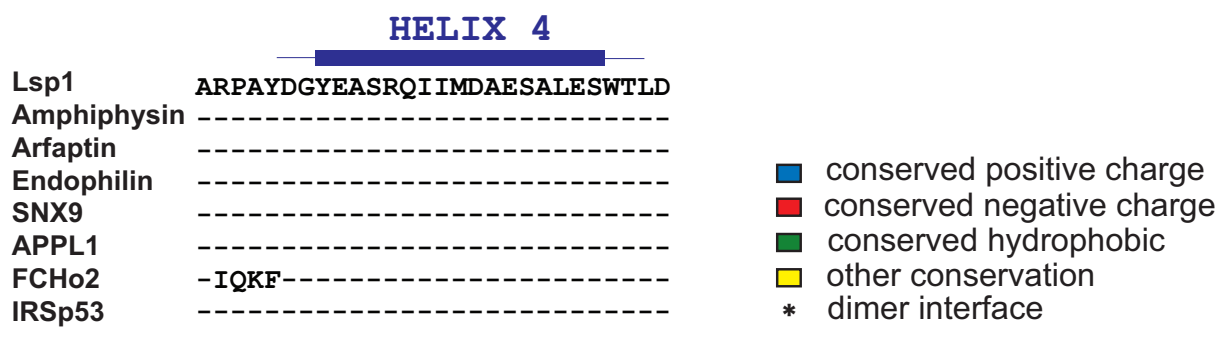

Supplementary Fig.2. Lsp1 and other BAR domain proteins show low sequence similarity. Structure based sequence alignment of BAR domains of S. cerevisiae Lsp1 (pdb code: 3plt), D. melanogaster amphiphysin (pdb code: 1uru), H. sapiens arfaptin 2 (pdb code: 1i49), H. sapiens endophilin 3 (pdb code: 2z0v), H. sapiens SNX9 (pdb code: 2raj), H. sapiens APPL1 (pdb code: 2q13), H. sapiens FCHo2 (pdb code: 2v0o), H. sapiens IRSp53 (pdb code: 1y2o). 
HELIX 1

S. cerevisiae Lsp1 ASIA C. albicans EEQ42705.1

S. pombe NP_587786.1 $U$. maydis XP- 762228.1

M. globosa XP_001730401.1

L. bicolor XP_001875537.1

P. placenta XP 002475915.1

C. neoformans XP 571806.1

P. graminis EFPP3628.1

A. capsulatus EEH05814.1

A. nidulans XP_661535.1

S. cerevisiae Lsp1 ASIA

C. albicans EEQ42705.1

S. pombe NP_587786.1

U. maydis XP 762228.1

M. globosa XP 001730401.1

L. bicolor XP-001875537.1

P. placenta XP_002475915.1

C. neoformans XP_571806.1

P. graminis EFP83628.1

A. capsulatus EEH05814.1

A. nidulans XP_661535.1

S. cerevisiae Lsp1 ASIA C. albicans EEQ42705.1

S. pombe NP_587786.1

$U$. maydis XP_762228.1

M. globosa XP_001730401.1

L. bicolor XP 001875537.1

P. placenta XP_002475915.1

C. neoformans XP_571806.1

P. graminis EFPP3628.1

A. capsulatus EEH05814.1

A. nidulans XP_661535.1

S. cerevisiae Lsp1 ASIA C. albicans EEQ42705.1

S. pombe NP 587786.1

U. maydis XP_762228.1

M. globosa XP_001730401.1

L. bicolor XP 001875537.1

$P$. placenta XP 002475915.1

C. neoformans XP 571806.1

P. graminis EFP83628.1

A. capsulatus EEH05814.1

A. nidulans XP_661535.1
GPELARKLSQLVKTEKGVLRAMEVVASERREAAKQLSLWGA GPELSRKLSQFIKMEKNFMRAVEVTSRERKDVAKQLSAWGF -PDLAKRLAVLVKMEKNVMRSMEVTIRGRRDCAROLSYWG GNODLROLODVITSEKSFIOSNTKTAADFKKNAEAIKAWSA GNSDLKQLQEMIHREKAFVAANAKTATE IQLSSDALRAWGSN GNTELRPLQDLITAEKAVLILIQKLSVDYSKAAEALRVWGTS -NKDLRTLQELITAEKSVLNSLQRLSADLVKASEALKAWGIG GHKDLRALODI ISSEKGVLOMSERLAAETSKACSFLPPYGTO APKDIKNLAEI ISTEKSVLSTSSKLSVDYRKAAEALKEWGLN -PELCRRLYKLIKAENHAIGSYENAGRDRVSIASQLSDWGES -PQLSKKMNRVIKSENSAIAAHESAARQRMSIAAQISEWGET

HELIX 2

\section{HELIX 3}

S----------TKI PVLEOELVRAEAESLVAEAOLSNITREKLKAAYSYMFDSLRELSEKFALIAGYGKALLELIDDSPVTPGF S---------PKI PVLEQELVRAEAESLVAEAQLSNITREQLKAAFNYQFDATRELAEKYALIAGYGKALLELLDDSAVTPGE S----------PRLVTMEQELVREEAACLVAEAQLSNTTREKFKQAMT FNLDALHEHAEKLNL IATYGRHLLNLIDDTPVTPGE MGPENKELMKVTA QLKEMRGEMEALHVEVINENAAIGDFKRRTVKEALGIKSGALLEMAEKITILAE ISKLMLEEVPLQPTRPGM MGPENKDLAKVTSSLRELRSDMEVLNNEMAYEFAALGDYKRRTIVEALSLKSGGLMELAEKSIVIAESCRLLVEEVPLIPTVPNE MGSEHKHLOVONELLNRLNAEIRAMDGEIMSEETGLGDFKRSTVRVVMGLKFGGLVECCEKGVIVGEYGKLVTAE I PEETTQPGT MSPDNKNLQVQTDLLNKLRDEIOIMDAD IMAEEASLGDYKRTSAKAWMGLKFGGLAECSEKGVIIGEFGKMLVAEIPLDTTEPGI MGPENKSLPHQTELLERLRSDMRQMDQD ITTEETKIGDFKRQTLKEALSYKFGGLEELGEKMCI I GELGKLLLEEVPLEETPVGY MSSENKDLPALTTRLQEARSELISLENSVAIEDARLSDFKRETVREGLGLRLGAMLELAEKMTIVCEFGKMLTNEVPIERTPPGA N------- PKLVTLEQELVRAEA QMLVAEAQLTNITRQKLKEAFD IHLAAT IERAEKQI ILARHGRRLINILDDTPLVPGD S----------HKIETMEQELVRAEAQNLVAEAQLTNVTRQRFKEAYSVHLAAVIERGEKQALLARHARRLINCLDDTSVIPGD

HELIX 4

ARPAYDGYEASRQI IMDAESALFSWTLD

TRPAYDGYEASKQI I IDAENALASWTLD

ARPAYDGYETSRQIVMDAEHALSSWVP-

PRAEYHSFAKTESLLQQATRAIAD----

GRAPYRNEARTNRLLQEAVRQLESITFE

PRSLYYGHAKTESLLVEATRALSDVQL-

PRSVYQGHSNTEALVADARRALTN----

GRAPYTGYEKTENAVREATKCLGTVQF-

ARAPYCAANKTQAVVEEAQRCLT-----

TRSAYEQMDQARQVLNDAEQDLRGWEP-

EPKPYERGNDAKQIVEDAERELQSWETD

Supplementary Fig.3. Prediction of Lsp1/Pil1 like proteins containing BAR domains. 


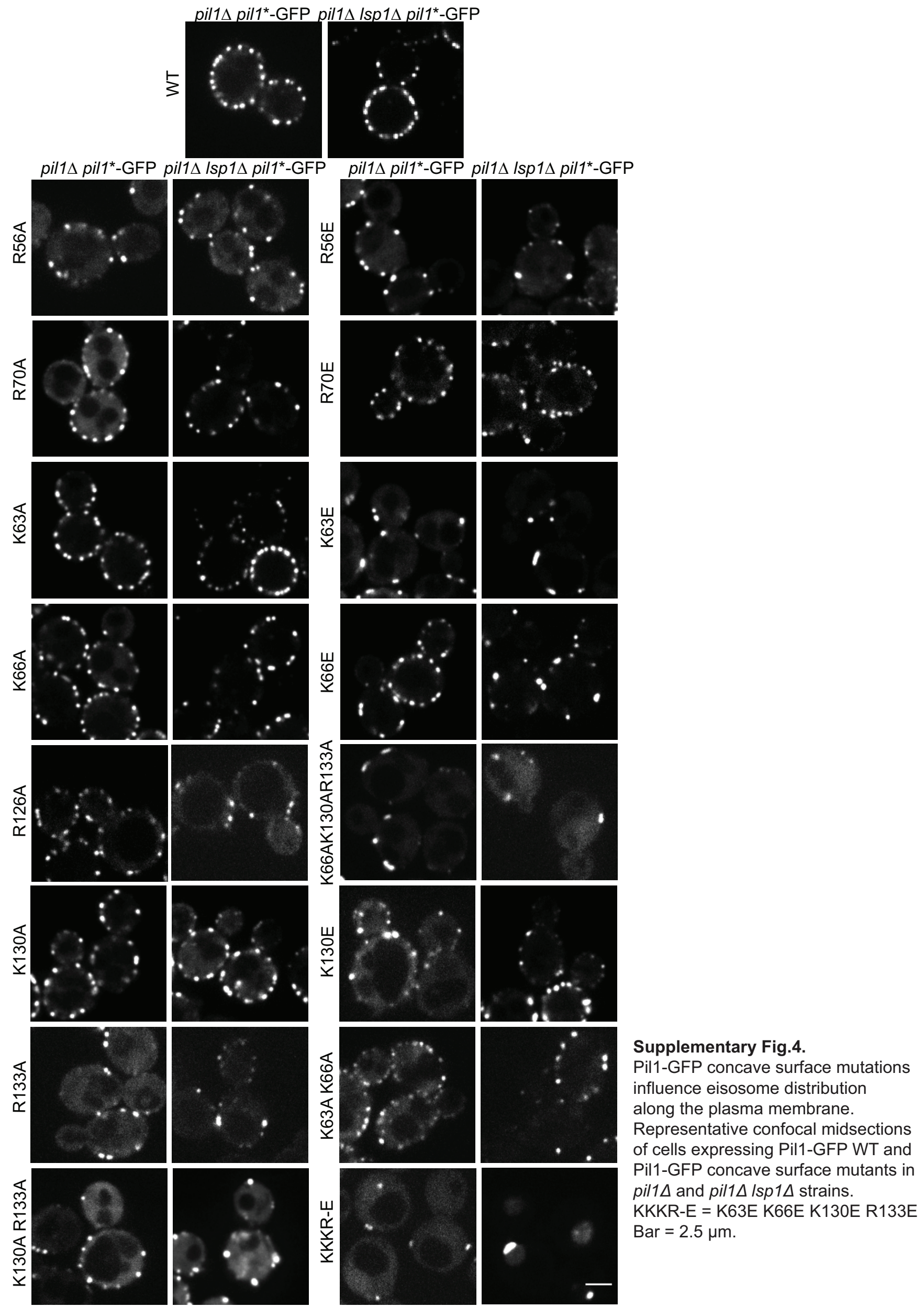

Nature Structural \& Molecular Biology: doi:10.1038/nsmb.2080 
a

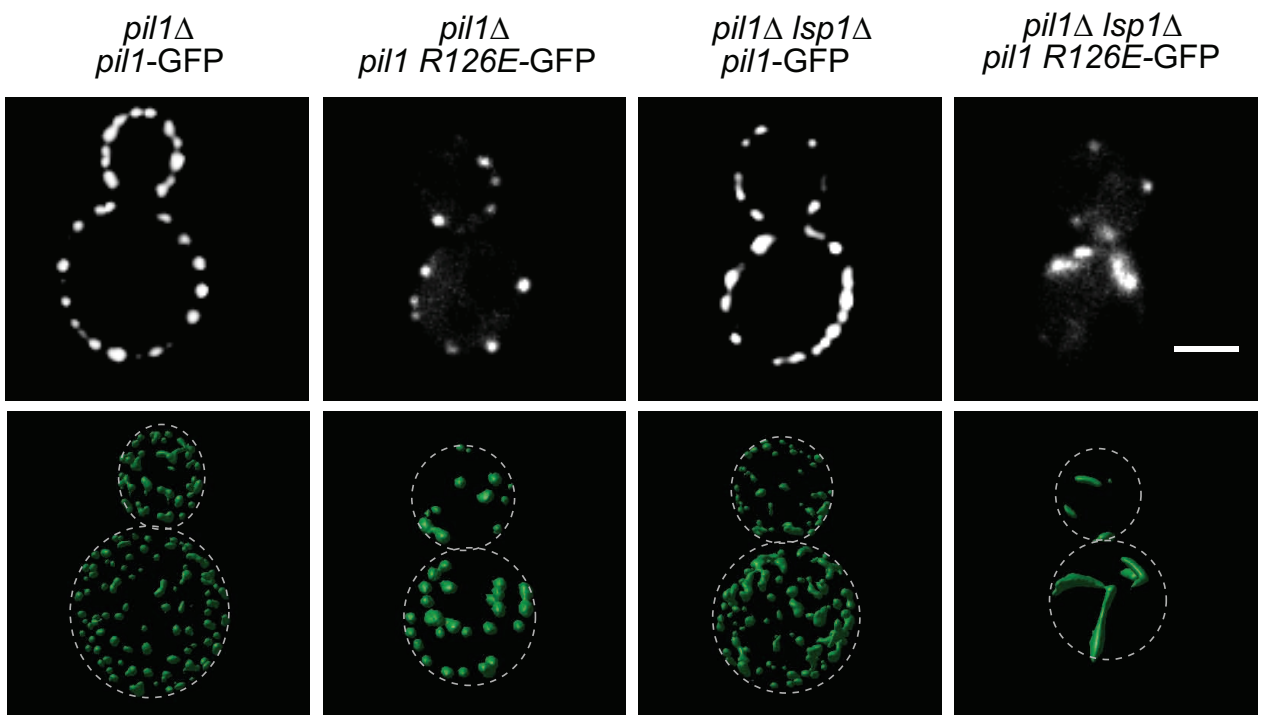

b
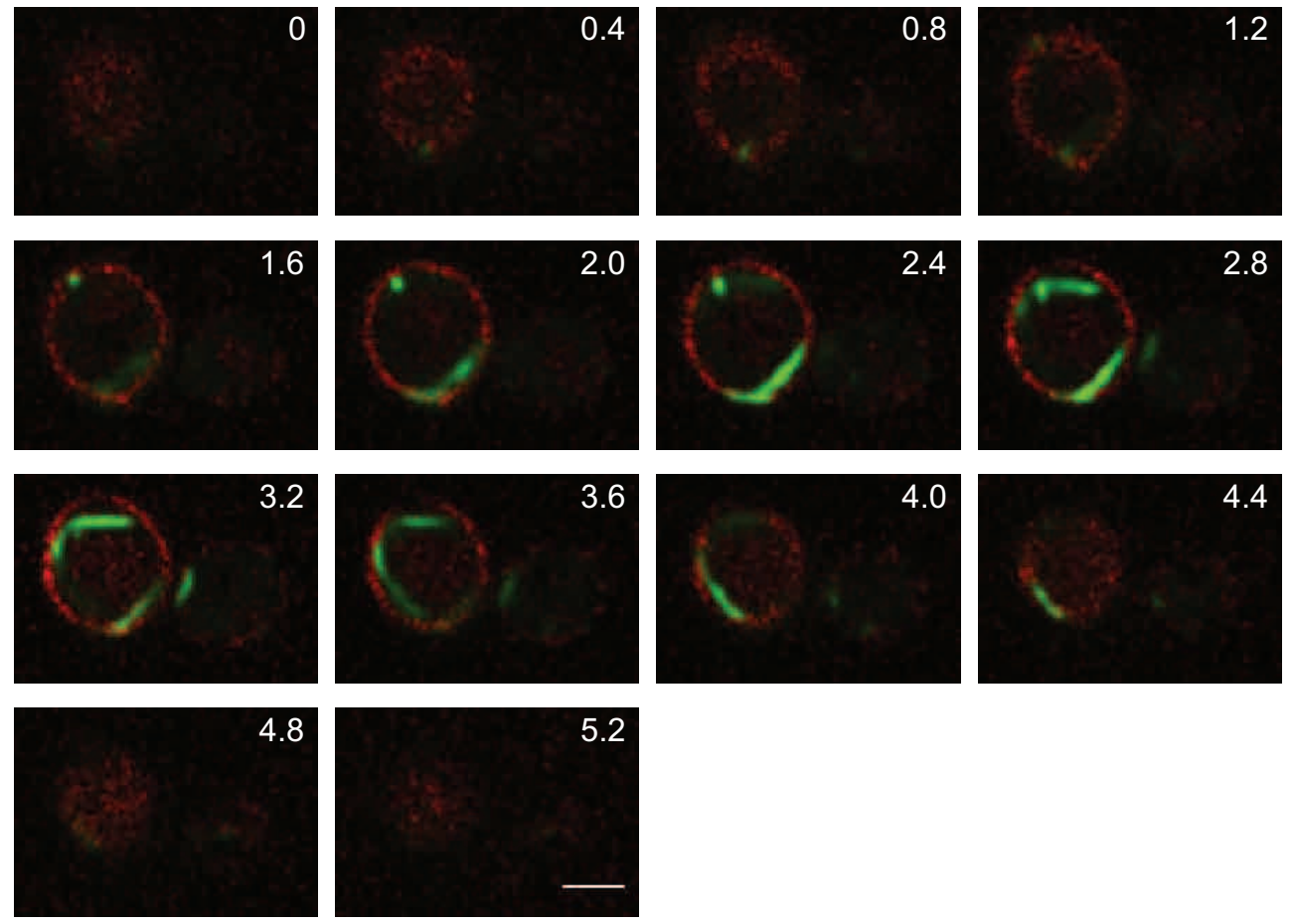

\section{Supplementary Fig.5.}

Pil1-GFP R126E in pil1 $/ s p 1 \Delta$ strain forms long rods traversing the cytoplasm.

(a) Representative confocal midsections and 3D reconstructions of cells expressing Pil1-GFP WT and Pil1-GFP mutant R126E in pil1 $\Delta$ and pil1 $\Delta / s p 1 \Delta$ strains

(b) Z-stack images of cells expresing Pil1-GFP R126E in pil1 $\Delta$ lsp1 $\Delta$ collected at $0.4 \mu \mathrm{m}$ distances. Ylr413w-RFPmars used as a membrane staining marker. Bar $=2.5 \mu \mathrm{m}$. 

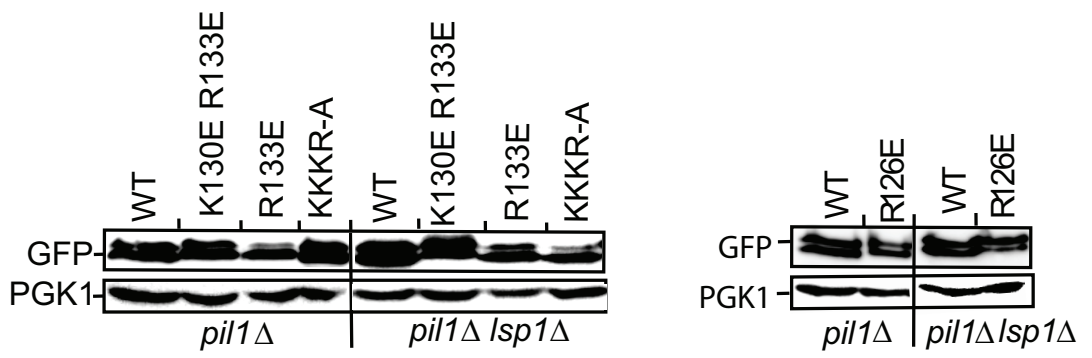

Supplementary Fig.6.

Expression levels of Pil1-GFP concave surface mutants.

Western blot analysis of Pil1-GFP concave surface mutants expressed in pil1 $\Delta$ and pil1 $\Delta$ lsp $1 \Delta$ strains.

PGK1 is shown as a loading control.
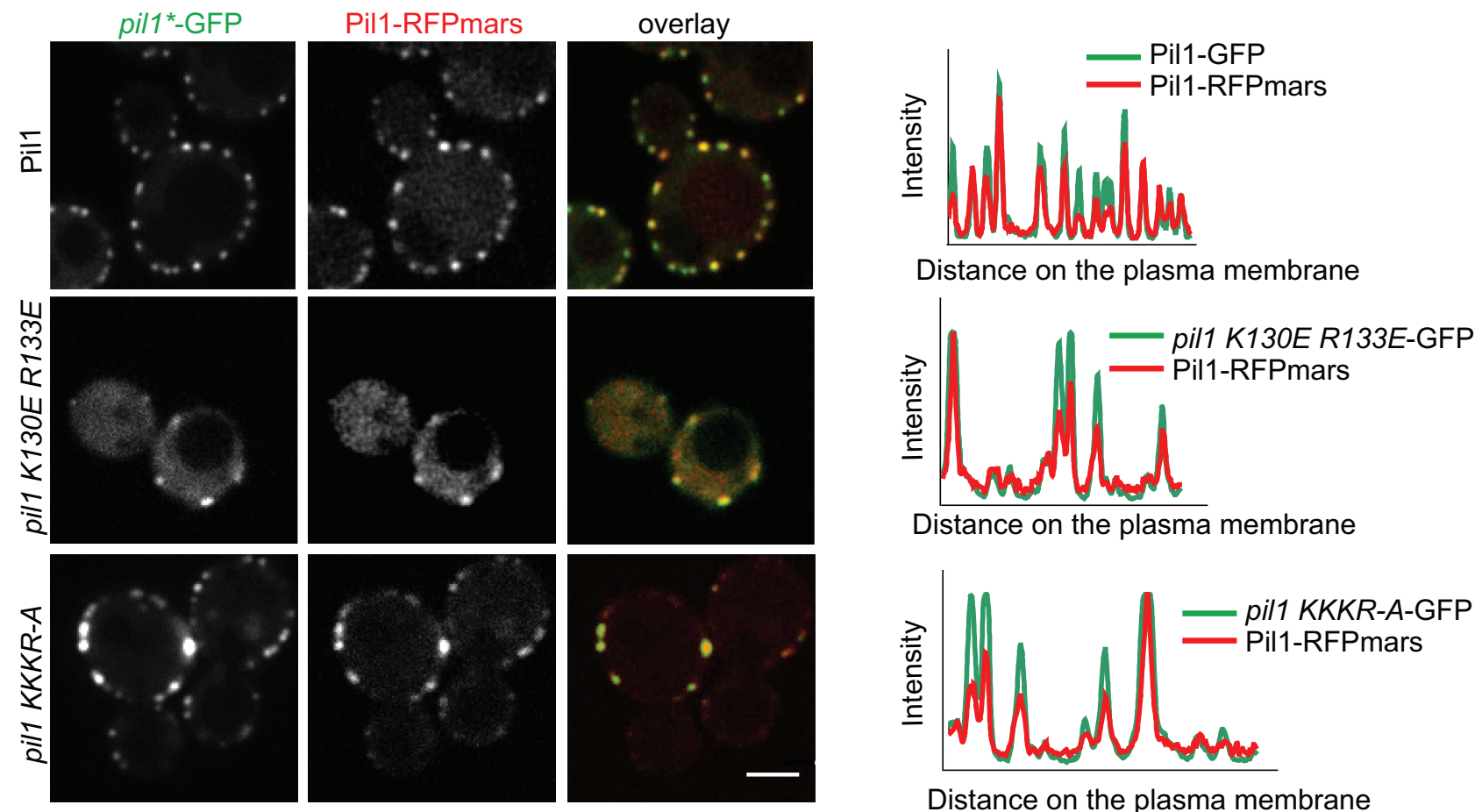

Distance on the plasma membrane

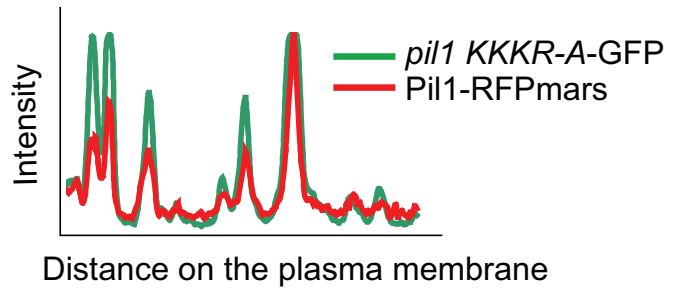

\section{Supplementary Fig.7.}

Concave surface mutants of Pil1 are dominant on WT in vivo.

Representative confocal midsections of cells expressing Pil1-GFP concave surface mutants and Pil1-RFPmars WT $(B a r=2.5 \mu \mathrm{m})$ and intensity profiles of Pil1-GFP concave surface mutants and Pil1-RFPmars WT along the plasma membrane. 

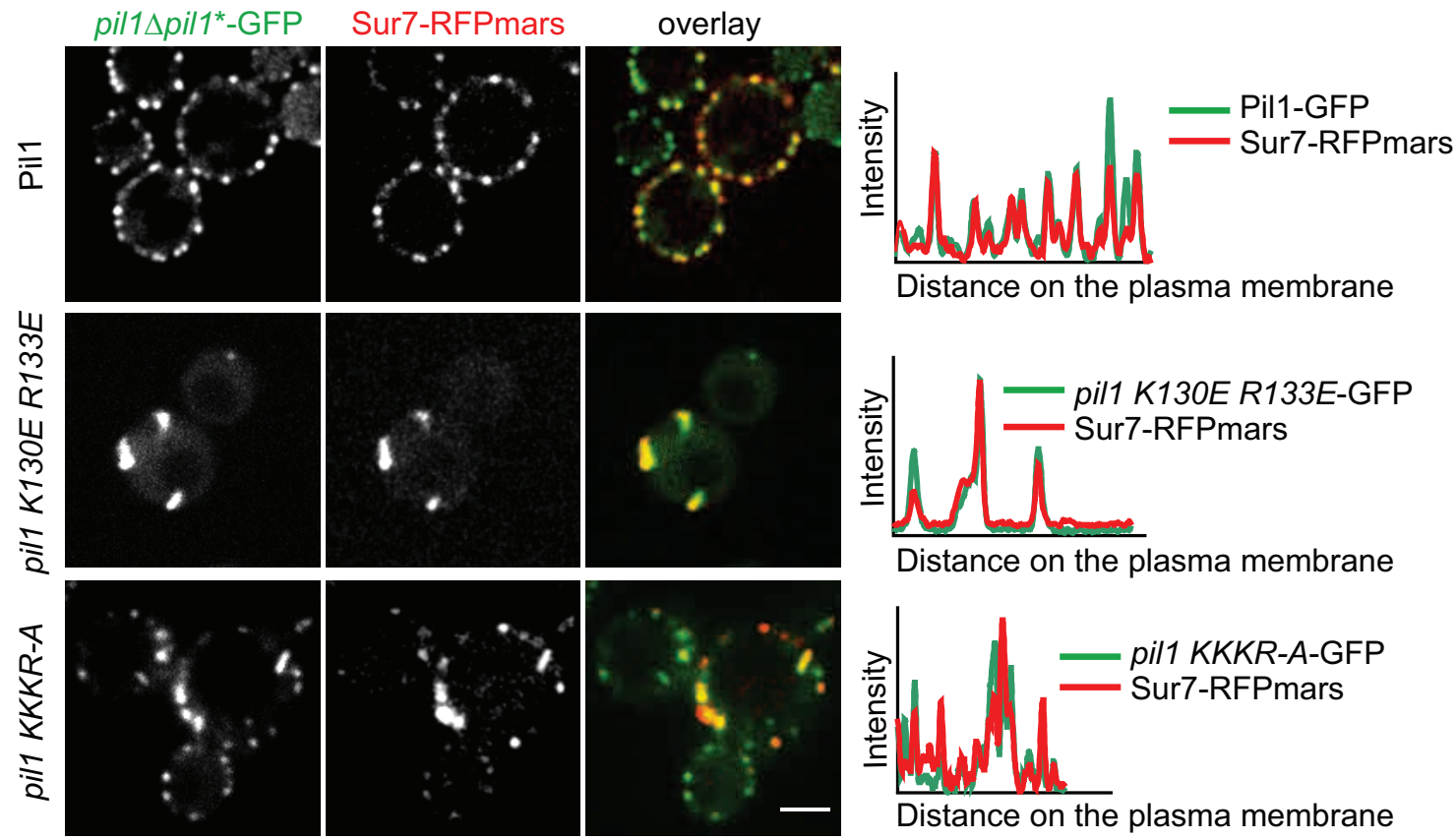

\section{Supplementary Fig.8.}

The positive patch of Pil1 is required for normal plasma membrane organization.

Representative confocal midsections of cells expressing Pil1-GFP concave surface mutants and Sur7-RFPmars (left). Bar $=2.5 \mu \mathrm{m}$. Intensity profiles of Pil1-GFP concave surface mutants and Sur7-RFPmars along the plasma membrane (right). 
Supplementary Table 1. Data collection, phasing and refinement statistics.

\begin{tabular}{|c|c|c|c|}
\hline \multirow{2}{*}{ Data collection } & \multirow[t]{2}{*}{ Native } & \multicolumn{2}{|c|}{ SeMet } \\
\hline & & & \\
\hline Space group & $\mathrm{C} 2$ & $\mathrm{C} 2$ & $\mathrm{C} 2$ \\
\hline \multicolumn{4}{|l|}{ Cell dimensions } \\
\hline$a, b, c(\AA)$ & $273.16,38.75,75.51$ & $274.93,38.68,75.08$ & $276.91,38.84,75.50$ \\
\hline \multirow[t]{2}{*}{$\beta\left(^{\circ}\right)$} & 99.3 & 99.3 & 99.3 \\
\hline & & Peak & Inflection \\
\hline Wavelength & 0.97970 & 0.97855 & 0.97912 \\
\hline Resolution $(\AA)$ & 2.9 & 2.9 & 3.2 \\
\hline$R_{\text {merge }}$ & $10.6(89.6)$ & $11.6(70.0)$ & $11.5(74.0)$ \\
\hline$I / \sigma I$ & $18.75(1.91)$ & $13.63(1.11)$ & $16.94(1.61)$ \\
\hline Completeness $(\%)$ & $99.6(99.8)$ & $96.4(91.4)$ & $98.5(93.7)$ \\
\hline Redundancy & $6.8(6.5)$ & $5.0(3.9)$ & $6.4(4.2)$ \\
\hline \multicolumn{4}{|l|}{ Refinement } \\
\hline Resolution $(\AA)$ & 2.9 & & \\
\hline No. of unique reflections & 17725 & & \\
\hline$R_{\text {work }} / R_{\text {free }}$ & $23.6 / 29.6$ & & \\
\hline \multicolumn{4}{|l|}{ No. atoms } \\
\hline Protein & 5031 & & \\
\hline Water & 6 & & \\
\hline \multicolumn{4}{|l|}{$B$-factors } \\
\hline Protein & 63.92 & & \\
\hline Water & $18.63 *$ & & \\
\hline \multicolumn{4}{|l|}{ R.m.s deviations } \\
\hline Bond lengths $(\AA)$ & 0.010 & & \\
\hline Bond angles $\left(^{\circ}\right)$ & 0.001 & & \\
\hline
\end{tabular}

Values in parentheses are for highest-resolution shell

* Since only a few well-ordered water molecules are included in the structure their average B-factor is lower than the average $\mathrm{B}$-factor for the protein 


\section{Supplementary Table 2. Yeast strains used in this study.}

\section{Strain Genotype}

\section{Reference}

\begin{tabular}{|c|c|c|}
\hline TWY138 & Mata ura3 trp1 leu2 his3 ade2 can1-100 & 23 \\
\hline TWY226 & Matā ura3 trp1 leu2 his3 ade2 can1-100, pil14::KAN & 23 \\
\hline TWY232 & 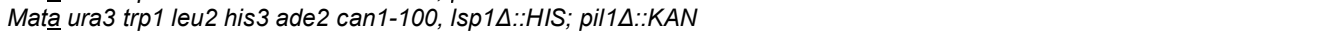 & 23 \\
\hline TWY1988 & Mata ura3 trp1 leu2 his3 ade2 can1-100, pil14::KAN, PIL1-GFP::URA pRS306 - integrated & This study \\
\hline TWY1987 & Matāa ura3 trp1 leu2 his3 ade2 can1-100, pil1A::KAN, Isp1A::HIS PIL1-GFP::URA pRS306 - integrated & This study \\
\hline TWY2017 & Mata ura3 trp1 leu2 his3 ade2 can1-100, pil1A::KAN, pil1-GFP R56A::URA pRS306 - integrated & This study \\
\hline TWY2020 & Matā ura3 trp1 leu2 his3 ade2 can1-100, pil1A::KAN, Isp1A::HIS pil1-GFP R56A::URA pRS306 - integrated & This study \\
\hline TWY1963 & Matā ura3 trp1 leu2 his3 ade2 can1-100, pil14::KAN, pil1-GFP R56E::URA pRS306 - integrated & This study \\
\hline TWY1977 & Matā ura3 trp1 leu2 his3 ade2 can1-100, pil14::KAN, Isp14::HIS pil1-GFP R56E::URA pRS306 - integrated & This study \\
\hline TWY1968 & Matā ura3 trp1 leu2 his3 ade2 can1-100, pil14::KAN, pil1-GFP K63A::URA pRS306 - integrated & This study \\
\hline TWY1982 & Matā ura3 trp1 leu2 his3 ade2 can1-100, pil1A::KAN, Isp1A::HIS pil1-GFP K63A::URA pRS306 - integrated & This study \\
\hline TWY1970 & Matā ura3 trp1 leu2 his3 ade2 can1-100, pil1A::KAN, pil1-GFP K63E::URA pRS306 - integrated & This study \\
\hline TWY1984 & Matā ura3 trp1 leu2 his3 ade2 can1-100, pil14::KAN, Isp1A::HIS pil1-GFP K63E::URA pRS306 - integrated & This study \\
\hline TWY1969 & Matā ura3 trp1 leu2 his3 ade2 can1-100, pil1A::KAN, pil1-GFP K66A::URA pRS306 - integrated & This study \\
\hline TWY1983 & Mata ura3 trp1 leu2 his3 ade2 can1-100, pil1A::KAN, Isp1A::HIS pil1-GFP K66A::URA pRS306 - integrated & This study \\
\hline TWY1971 & Matä ura3 trp1 leu2 his3 ade2 can1-100, pil1A::KAN, pil1-GFP K66E::URA pRS306 - integrated & This study \\
\hline TWY1985 & Mata ura3 trp1 leu2 his3 ade2 can1-100, pil1A::KAN, Isp1A::HIS pil1-GFP K66E::URA pRS306 - integrated & This study \\
\hline TWY1964 & Matā ura3 trp1 leu2 his3 ade2 can1-100, pil1A::KAN, pil1-GFP R70A::URA pRS306 - integrated & This study \\
\hline TWY1978 & Mata ura3 trp1 leu2 his3 ade2 can1-100, pil1A::KAN, Isp1A::HIS pil1-GFP R70A::URA pRS306 - integrated & This study \\
\hline TWY2206 & Matā ura3 trp1 leu2 his3 ade2 can1-100, pil14::KAN, pil1-GFP R70E::URA pRS306 - integrated & This study \\
\hline TWY2169 & Matā ura3 trp1 leu2 his3 ade2 can1-100, pil14::KAN, Isp1A::HIS pil1-GFP R70E::URA pRS306 - integrated & This study \\
\hline TWY2201 & Mata ura3 trp1 leu2 his3 ade2 can1-100, pil14::KAN, pil1-GFP R126A::URA pRS306 - integrated & This study \\
\hline TWY2200 & Matā ura3 trp1 leu2 his3 ade2 can1-100, pil14::KAN, Isp14::HIS pil1-GFP R126A ::URA pRS306 - integrated & This study \\
\hline TWY2289 & Mata ura3 trp1 leu2 his3 ade2 can1-100, pil14::KAN, pil1-GFP R126E::URA pRS306 - integrated & This study \\
\hline TWY2290 & Mata ura3 trp1 leu2 his3 ade2 can1-100, pil14::KAN, Isp1 $::$ HIS pil1-GFP R126E::URA pRS306 - integrated & This study \\
\hline TWY1967 & Mata ura3 trp1 leu2 his3 ade2 can1-100, pil1A::KAN, pil1-GFP K130A::URA pRS306 - integrated & This study \\
\hline TWY1981 & Matā ura3 trp1 leu2 his3 ade2 can1-100, pil14::KAN, Isp14::HIS pil1-GFP K130A ::URA pRS306 - integrated & This study \\
\hline TWY1965 & Matā ura3 trp1 leu2 his3 ade2 can1-100, pil14::KAN, pil1-GFP K130E::URA pRS306 - integrated & This study \\
\hline TWY1979 & Mata ura3 trp1 leu2 his3 ade2 can1-100, pil14::KAN, Isp14::HIS, pil1-GFP K130E::URA pRS306 - integrated & This study \\
\hline TWY2172 & Matā ura3 trp1 leu2 his3 ade2 can1-100, pil14::KAN, pil1-GFP R133A::URA pRS306 - integrated & This study \\
\hline TWY2173 & Mata ura3 trp1 leu2 his3 ade2 can1-100, pil14::KAN, Isp14::HIS pil1-GFP R133A::URA pRS306 - integrated & This study \\
\hline TWY1965 & Mata ura3 trp1 leu2 his3 ade2 can1-100, pil14::KAN, pil1-GFP K130E::URA pRS306 - integrated & This study \\
\hline TWY1980 & Mata ura3 trp1 leu2 his3 ade2 can1-100, pil1A::KAN, Isp1A::HIS pil1-GFP R133E::URA pRS306 - integrated & This study \\
\hline TWY2170 & Mata ura3 trp1 leu2 his3 ade2 can1-100, pil14::KAN, pil1-GFP K63A K66A::URA pRS306 - integrated & This study \\
\hline TWY2171 & Matā ura3 trp1 leu2 his3 ade2 can1-100, pil14::KAN, Isp1A::HIS pil1-GFP K63A K66A::URA pRS306 - integrated & This study \\
\hline TWY1960 & Mata ura3 trp1 leu2 his3 ade2 can1-100, pil1A::KAN, pil1-GFP K63E K66E::URA pRS306 - integrated & This study \\
\hline TWY1974 & Mata ura3 trp1 leu2 his3 ade2 can1-100, pil1A::KAN, Isp1A::HIS pil1-GFP K63E K66E::URA pRS306 - integrated & This study \\
\hline TWY1961 & Matāa ura3 trp1 leu2 his3 ade2 can1-100, pil14::KAN, pil1-GFP K130A R133A ::URA pRS306 - integrated & This study \\
\hline TWY1975 & Matā ura3 trp1 leu2 his3 ade2 can1-100, pil14::KAN, Isp1A::HIS pil1-GFP K130A R133A ::URA pRS306 - integrated & This study \\
\hline TWY1962 & Mata ura3 trp1 leu2 his3 ade2 can1-100, pil1A::KAN, pil1-GFP K130E R133E ::URA pRS306 - integrated & This study \\
\hline TWY1976 & Mata ura3 trp1 leu2 his3 ade2 can1-100, pil14::KAN, Isp1A::HIS pil1-GFP K130E R133E ::URA pRS306 - integrated & This study \\
\hline TWY2018 & Matā ura3 trp1 leu2 his3 ade2 can1-100, pil14::KAN, pil1-GFP K66A K130A R133A::URA pRS306 - integrated & This study \\
\hline TWY2021 & Matā ura3 trp1 leu2 his3 ade2 can1-100, pil14::KAN, Isp1A::HIS pil1-GFP K66A K130A R133A::URA pRS306 - integrated & This study \\
\hline TWY1972 & Matā ura3 trp1 leu2 his3 ade2 can1-100, pil14::KAN, pil1-GFP K63A K66A K130A R133A::URA pRS306 - integrated & This study \\
\hline TWY1986 & $\begin{array}{l}\text { Matā ura3 trp1 leu2 his3 ade2 can1-100, pil14::KAN, Isp1A::HIS pil1-GFP K63A K66A K130A R133A::URA pRS306 - } \\
\text { integrated }\end{array}$ & This study \\
\hline TWY2019 & Mata ura3 trp1 leu2 his3 ade2 can1-100, pil14::KAN, pil1-GFP K63E K66E K130E R133E::URA pRS306 - integrated & This study \\
\hline TWY2022 & $\begin{array}{l}\text { Mata ura3 trp1 leu2 his3 ade2 can1-100, pil14::KAN, Isp14::HIS pil1-GFP K63E K66E K130E R133E::URA pRS306 - } \\
\text { integrated }\end{array}$ & This study \\
\hline TWY1649 & Mata ura3 trp1 leu2 his3 ade2 can1-100, PIL1-RFPmars::NAT & [unpublished data] \\
\hline TWY2312 & Mata ura3 trp1 leu2 his3 ade2 can1-100, PIL1-RFPmars::NAT, pil1A::KAN, PIL1-GFP::URA pRS306 - integrated & This study \\
\hline TWY2313 & $\begin{array}{l}\text { Mata ura3 trp1 leu2 his3 ade2 can1-100, PIL1-RFPmars::NAT, pil14::KAN, pil1-GFP K130E R133E::URA pRS306 - } \\
\text { integrated }\end{array}$ & This study \\
\hline TWY2314 & $\begin{array}{l}\text { Mata ura3 trp1 leu2 his3 ade2 can1-100, PIL1-RFPmars:nat, pil14::KAN, pil1-GFP K63A K66A K130A R133A::URA } \\
\text { pRS306 - integrated }\end{array}$ & This study \\
\hline TWY1541 & Mata ura3 trp1 leu2 his3 ade2 can1-100, PMA1-GFP.:HIS, SUR7-RFPmars::NAT & This study \\
\hline TWY2307 & Mata ura3 trp1 leu2 his3 ade2 can1-100, SUR7-RFPmars::NAT, pil14::KAN, PIL1-GFP::URA & This study \\
\hline TWY2308 & Mata ura3 trp1 leu2 his3 ade2 can1-100, SUR7-RFPmars::NAT, pil14::KAN, pil1-GFP K130E R133E::URA & This study \\
\hline TWY2309 & Matā ura3 trp1 leu2 his3 ade2 can1-100, SUR7-RFPmars::NAT, pil1A::KAN, pil1-GFP K63A K66A K130A R133A::URA & This study \\
\hline TWY2291 & Mata ura3 trp1 leu2 his3 ade2 can1-100, pil14::HIS pan1-20 ts pRS316-PIL1[202]::URA & This study \\
\hline TWY2292 & Matā ura3 trp1 leu2 his3 ade2 can1-100, pil14::HIS pan1-20 ${ }^{t s}$ pRS316-PIL1[202]::URA pRS315-PIL1-GFP::LEU & This study \\
\hline TWY2294 & $\begin{array}{l}\text { Matāa ura3 trp1 leu2 his3 ade2 can1-100, pil14::HIS pan1-20 }{ }^{t s} \text { pRS316-PIL1[202]::URA pRS315-pil1-GFP K130E } \\
\text { R133E::LEU }\end{array}$ & This study \\
\hline TWY2295 & Mata ura3 trp1 leu2 his3 ade2 can1-100, pil14::HIS pan1-20ts pRS316-PIL1[202]::URA pRS315-pil1-GFP R133E::LEU & This study \\
\hline TWY2296 & $\begin{array}{l}\text { Mata ura3 trp1 leu2 his3 ade2 can1-100, pil14::HIS pan1-20 } 0^{\text {ts }} \text { pRS316-PIL1[202]::URA pRS315-pil1-GFP K63A K66A } \\
\text { K130A R133A::LEU }\end{array}$ & This study \\
\hline TWY2404 & Mata ura3 trp1 leu2 his3 ade2 can1-100, pil14::HIS pan1-20 pRS316-PIL1[202]::URA pRS315-pil1-GFP R126E::LEU & This study \\
\hline TWY2446 & $\begin{array}{l}\text { Mata ura3 trp1 leu2 his3 ade2 can1-100, pil1A::KAN, Isp1A::HIS pil1-GFP R126E::URA pRS306 - integrated, YLR413W- } \\
\text { RFPmars::NAT }\end{array}$ & This study \\
\hline
\end{tabular}




\section{Supplementary Methods}

\section{Cloning, protein expression and purification}

Lsp1 ASIA from S. cerevisiae was cloned using Seamless Ligation Independent Cloning ${ }^{1}$ to a modified $\mathrm{pET}$ vector that introduced a hexahistidine affinity tag and a PreScission protease site to the $\mathrm{N}$ terminus of the translated protein. Lsp1 ASIA was expressed in BL21 (DE3) Rosetta (Novagen). Expression was performed in a $1 \mathrm{~L}$ bioreactor at $37^{\circ} \mathrm{C}, \mathrm{pH}$ 7.0, $2 \mathrm{~L} \mathrm{~min}^{-1}, 800 \mathrm{rpm}$. SeMet derivative was expressed in minimal media with all amino acids supplemented except for methionine, which was replaced by SeMet. SeMet incorporation was confirmed by electrospray mass spectrometry. Proteins were purified from bacterial lysate using Ni-NTA Agarose (Qiagen). His-tag was cleaved off overnight with PreScission protease (provided by MPI-B Core Facility). Proteins were further purified on HiTrap Q Fast Flow anion exchange column (GE Healthcare) and next on a Superdex 75 gel-filtration column (GE Healthcare). Protein samples were concentrated up to $15 \mathrm{mg}$ $\mathrm{ml}^{-1}$ prior to crystallization.

\section{Protein crystallization, data collection, structure determination and refinement}

Crystallization was carried out by the hanging drop, vapor diffusion method ${ }^{2}$ Lsp1 ASIA native and SeMet derivative crystallized under identical conditions $(0.2 \mathrm{M} \mathrm{NaCl}, 20 \mathrm{mM}$ phenol, 20\% PEG 8000, 0.1M CAPS pH 11). $100 \mu$ of parafinum oil was spread on the surface of the reservoir solution to slow down vapor diffusion, reduce the number of crystals grown and increase their size ${ }^{3}$. Diffraction quality crystals reaching the size $0.40 \mathrm{x}$ $0.05 \times 0.01 \mathrm{~mm}$ were obtained after 5 days at room temperature. Before flash freezing, the crystals were transferred into a cryoprotectant solution containing $10 \%$ ethylene glycol. Xray data were collected at $100 \mathrm{~K}$ at the X10SA (PXII) beamline, the Swiss Light Source (SLS), Villigen, Switzerland on a MAR 225 CCD detector. Native and SeMet reflection data were indexed, integrated, and scaled with HKL-3000 (4). The structure was solved using multiple-wavelength anomalous diffraction (MAD) with HKL-3000 (4), which is integrated with MLPHARE ${ }^{5}, \mathrm{DM}^{6}, \mathrm{SHELXD}^{7}, \mathrm{SHELXE}^{8}, \mathrm{CCP}^{4}$ (9), SOLVE${ }^{10}, \mathrm{RESOLVE}^{11}$ and ARP/WARP ${ }^{12}$. The initial model of the native structure was obtained by molecular replacement using MOLREP ${ }^{13}$. The model was subjected to iterative rounds of manual rebuilding in $\mathrm{COOT}^{14}$ and refinement using REFMAC5 (15) utilizing data extending to 2.9 
$\AA$ resolution. $R_{\text {free }}$ was monitored by using $5 \%$ of the reflections as a test set. TLS restraints $^{16}$ were used in refinement. Ramachandran statistics calculated using MOLPROBITY ${ }^{17}$ shows that the refined structure has $99.8 \%$ of all residues in allowed regions and $95.8 \%$ of all residues in favored regions. The atomic coordinates and structure factors are deposited in the Protein Data Bank with the accession code 3plt. Supplementary Table 1 contains details regarding data collection and refinement statistics.

\section{Three-dimensional protein structure homology modeling}

The sequence alignment of Pil1 with Lsp1 was used for protein homology modeling. Theoretical models of the structures of Pil1 homodimer and Lsp1-Pil1 heterodimer were calculated using MODELLER ${ }^{18}$.

\section{Lsp1 phylogenetic tree preparation}

The tree was prepared using structure based sequence alignment of the $S$. cerevisiae Lsp1 ASIA (pdb code: 3plt) with the D. melanogaster Amphiphysin (pdb code: 1uru), $H$. sapiens Bin1/Amphiphysin 2 (pdb code: 2fic), $H$. sapiens Arfaptin 2 (pdb code: 1i49), $H$. sapiens Endophilin 2/Endophilin A1 (pdb code: 1x03), H. sapiens Endophilin 3 (pdb code: 2z0v), H. sapiens SNX9 (pdb code: 2raj), H. sapiens APPL1 (pdb code: 2q13), Galdieria sulfuraria BAR - red algae BAR protein (pdb code: 3caz), H. sapiens FCHo2 (pdb code: 2v0o), H. sapiens Pascin 1 (pdb code: 3hah), H. sapiens Pascin 2 (pdb code: 3haj), $H$. sapiens FNBP1 (pdb code: 2efl), H. sapiens CIP4 (pdb code: 2efk), S. cerevisiae Syp1 (pdb code: 3g9g), H. sapiens IRSp53 (pdb code: 1y2o). The alignment generated by DALI server ${ }^{19}$ was given to Geneious software (version 3.5.6) for the tree construction using neighbor-joining method, without any out-group or bootstrapping.

\section{Prediction of Lsp1/Pil1 like proteins containing BAR domains}

Candidate BAR domains were identified using repeated iterations of PSI-BLAST (www.ncbi.nlm.nih.gov/BLAST) against Lsp1 ASIA sequence with default parameters. Top 150 proteins were categorized according to phylum and checked for $\alpha$-helical content using secondary structure prediction tools available at http://us.expasy.org/tools/. 


\section{Projection of evolutionary conservation scores of amino acids on protein structure}

ConSurf $^{20}$ and Pymol $^{21}$ were used to prepare the Lsp1 ASIA dimer surface representation of amino acid evolutionary conservation (Fig. 2c).

\section{Yeast strains, plasmids and mutants}

All yeast stains were generated in the W303 background. PIL1-GFP gene was cloned into pRS306 and mutated using site-directed mutagenesis. Wild type PIL1-GFP and pil1-GFP concave surface mutants were transformed to TWY226 and TWY232 strains. Pil1 concave surface GFP mutant strains with Sur7-RFPmars were generated by crossing with TWY1541 strain. Pil1-GFP concave surface mutant strains with Pil1-RFPmars were generated by transformation to TWY1649. TWY2446 with pil1R126E-GFP mutant and Ylr413w-RFPmars was generated using the versatile toolbox for PCR-based tagging of yeast genes ${ }^{22}$. Yeast strains expressing Pil1-GFP concave surface mutants tested for growth on 5-FOA plate were generated in pRS306 than digested, ligated to pRS315, transformed to TWY2291 strain and grown at $24^{\circ} \mathrm{C}$. Supplementary Table 2 contains the list of the yeast strains used in this work.

\section{Microscopy}

For fluorescence microscopy, yeast cells were grown to an OD $=0.6$ in YPD at $30^{\circ} \mathrm{C}$. Cells were mounted in synthetic media onto cover slips previously coated with concanavalin $A$ and directly imaged with an ANDOR/TiLL iMIC CSU22 spinning disk confocal microscope, using an ANDOR iXonEM 897 back-illuminated EM CCD camera and an Olympus 100x 1.4 NA oil immersion objective. From this setup the images were collected using Andor Image $\mathrm{iQ} 1.9$ in the linear range of the camera. For presentation, images were cropped using ImageJ software (http://rsbweb.nih.gov/ij/) and for 3D reconstructions processed using Huygenes software (http://www.svi.nl/).

\section{Immunoblotting}

GFP and PGK1 were probed with anti-GFP antibody and anti-PGK1 antibody (Molecular Probes). 


\section{Supplementary References}

1. Li, M. Z. \& Elledge S. J. Harnessing homologous recombination in vitro to generate recombinant DNA via SLIC. Nat. Methods. 4, 251-256 (2007).

2. Wlodawer, A. \& Hodgson, K.O. Crystallization and crystal data of monellin. Proc. Natl. Acad. Sci. USA 72, 398-399 (1975).

3. Chayen, N.E. A novel technique to control the rate of vapor diffusion, giving larger protein crystals. J. Appl. Cryst. 30, 198-202 (1997).

4. Minor, W., Cymborowski, M., Otwinowski, Z. \& Chruszcz, M. HKL-3000: The Integration of Data Reduction and Structure Solution-From Diffraction Images to an Initial Model in Minutes. Acta Crystallogr. Sect. D: Biol. Crystallogr. 62, 859-866 (2006).

5. Otwinowski, Z. CCP4, SERC Daresbury Laboratory, Warrington, UK (1991).

6. Cowtan, K. DM: an automated procedure for phase improvement by density modification, Joint CCP4 and ESF-EACBM Newsletter on Protein Crystallography. 31, 34-38 (1994).

7. Schneider, T.R. \& Sheldrick, G.M. Substructure solution with SHELXD. Acta Crystallogr., Sect. D: Biol. Crystallogr. 58, 1772-1779 (2002).

8. Sheldrick, G.M. Macromolecular phasing with SHELXE. Z. Kristallogr. 217, 644-650 (2002).

9. CCP4. The CCP4 suite: programs for protein crystallography, Acta Crystallogr., Sect. D: Biol. Crystallogr. 50, 760-763 (1994).

10. Terwilliger, T.C. \& Berendzen J. Automated MAD and MIR structure solution. Acta Crystallogr., Sect. D: Biol. Crystallogr. 55, 849-861 (1999).

11. Terwilliger T.C. Automated structure solution, density modification and model building. Acta Crystallogr., Sect. D: Biol. Crystallogr. 58, 1937-1940 (2002).

12. Perrakis, A., Morris R. \& Lamzin V.S. Automated protein model building combined with iterative structure refinement. Nat. Struct. Biol. 6, 458-463 (1999).

13. Vagin, A.A. \& Teplyakov A., MOLREP: an Automated Program for Molecular Replacement. J. Appl. Cryst. 30, 1022-1025 (1997).

14. Emsley, P. \& Cowtan. K. Coot: model-building tools for molecular graphics. Acta Crystallogr. Sect. D: Biol. Crystallogr. 60, 2126-2132 (2004).

15. Murshudov, G.N., Vagin, A.A. \& Dodson, E.J. Refinement of macromolecular structures by the maximum-likelihood method. Acta Crystallogr. Sect. D: Biol. Crystallogr. 53, 240-255 (1997).

16. Jones, T.A., Zou, J.Y. \& Cowan S.W. and Kjeldgaard M. Improved methods for building protein models in electron density maps and the location of errors in these models. Acta Crystallogr., Sect. D: Biol. Crystallogr. 47, 110-119 (1991).

17. Davis, I.W., et al. Molprobity: all-atom contacts and structure validation for proteins and nucleic acids. Nucl. Acids Res. 35, 375-83 (2007).

18. Eswar, N., et al. Comparative protein structure modeling with MODELLER. Current Protocols in Bioinformatics, John Wiley \& Sons, Inc., Supplement 15, 5.6.1-5.6.3 (2006).

19. Holm, L., Kaariainen, S., Rosenstrom, P. \& Schenkel, A. Searching protein structure databases with DaliLite v.3. Bioinformatics 24, 2780-2781 (2008).

20. Ashkenazy, H., Erez, E., Martz, E., Pupko, T. \& Ben-Tal, N. ConSurf 2010: calculating evolutionary conservation in sequence and structure of proteins and nucleic acids. Nucl. Acids Res. 38, W529-W533 (2010).

21. De Lano, W.L. The Pymol Molecular Graphics System. DeLano Scientific, San Carlos CA, USA, www.pymol.org (2002).

22. Janke, C. et al. A versatile toolbox for PCR-based tagging of yeast genes: new fluorescent proteins, more markers and promoter substitution cassettes. Yeast 21, 947-962 (2004).

23. Walther, T.C. et al. Eisosomes mark static sites of endocytosis. Nature 439, 998-1003 (2006). 


\section{Supplementary Video Legend}

Pil1-GFP R126E in pil1 $/$ Isp $1 \Delta$ strain forms long rods traversing the cytoplasm.

YIr413w-RFPmars used as a membrane staining marker. Z-stack images collected at 0.2 $\mu \mathrm{m}$ distances. 



\title{
Eisosome proteins assemble into a membrane scaffold
}

\author{
Lena Karotki, ${ }^{1}$ Juha T. Huiskonen, ${ }^{2}$ Christopher J. Stefan, ${ }^{3}$ Natasza E. Ziółkowska, ${ }^{1}$ Robyn Roth, ${ }^{4}$ Michal A. Surma, ${ }^{5}$ \\ Nevan J. Krogan, ${ }^{6}$ Scott D. Emr, ${ }^{3}$ John Heuser ${ }^{4}$ Kay Grünewald, ${ }^{2}$ and Tobias C. Walther ${ }^{1,7}$ \\ 'Organelle Architecture and Dynamics, Max Planck Institute of Biochemistry, D-82152 Martinsried, Germany \\ ${ }^{2}$ Oxford Particle Imaging Centre, Division of Structural Biology, University of Oxford, Oxford OX3 7BN, England, UK \\ ${ }^{3}$ Weill Institute for Cell and Molecular Biology, Cornell University, Ithaca, NY 14853 \\ ${ }^{4}$ Department of Cell Biology and Physiology, Washington University, St. Lovis, MO 63110 \\ ${ }^{5}$ Max Planck Institute of Molecular Cell Biology and Genetics, 01307 Dresden, Germany \\ ${ }^{6}$ Department of Cellular and Molecular Pharmacology, University of California, San Francisco, San Francisco, CA 94158 \\ ${ }^{7}$ Department of Cell Biology, Yale University School of Medicine, New Haven, CT O6520
}

\section{Introduction}

Cells are delimited by the plasma membrane, which mediates all communication and transport in and out of the cell. This necessitates the coordinated execution of many biochemical reactions simultaneously. To achieve this intricate task, the plasma membrane is highly organized in space and time. Despite the importance of membrane domains in cell biology, the mechanisms involved in domain formation are not well understood in many cases. The plasma membrane of Saccharomyces cerevisiae is patterned into at least three distinct nonoverlapping domains that are named after specific marker proteins, including membrane compartment containing either Pma1 (MCP), target of rapamycin complex 2 (MCT), or Can1 (MCC; Malínská et al., 2003; Berchtold and Walther, 2009). These domains differ in appearance, forming either a punctate pattern (MCC and MCT) or a network (MCP). In addition to harboring

Correspondence to Tobias C. Walther: tobias.walther@yale.edu

Abbreviations used in this paper: DEEM, deep-etching EM; E-MAP, epistatic miniarray profile; IHRSR, iterative helical real-space reconstruction; PC, phosphatidylcholine; PI, phosphatidylinositol; PS, phosphatidylserine; YPD, yeast peptone dextrose.

subunits of two Bin-Amphiphysin-Rvs domain-containing proteins, Pill and Lsp1. In this paper, we show that these proteins self-assemble into higher-order structures and bind preferentially to phosphoinositide-containing membranes. Using a combination of electron microscopy approaches, we generate structural models of Pill and Lspl assemblies, which resemble eisosomes in cells. Our data suggest that the mechanism of membrane organization by eisosomes is mediated by self-assembly of its core components into a membrane-bound protein scaffold with lipid-binding specificity.

specific proteins, yeast plasma membrane domains also vary in lipid composition. In particular, the MCC is thought to be enriched in ergosterol, the major yeast sterol (Grossmann et al., 2007).

Ultrastructurally, MCCs appear as furrows in the plasma membrane (Strádalová et al., 2009). Their formation is mediated by large protein complexes underlying this domain, termed eisosomes (Walther et al., 2006). Eisosomes are remarkable cellular structures; they form a distributed pattern of complexes that are spaced at a minimal distance from each other. Moreover, they are extremely stable once formed and do not exchange subunits, nor do they move (Malínská et al., 2003; Walther et al., 2006). A typical yeast cell has 30 eisosomes, depending on its surface area, each of them consisting of many copies of two extremely abundant, highly similar proteins, Pil1 and Lsp1 (115,000 and 104,000 molecules per cell,

(C) 2011 Karotki et al. This article is distributed under the terms of an AttributionNoncommercial-Share Alike-No Mirror Sites license for the first six months after the publication date (see http://www.rupress.org/terms). After six months it is available under a Creative Commons License (Attribution-Noncommercial-Share Alike 3.0 Unported license, as described at http://creativecommons.org/licenses/by-nc-sa/3.0/). 
A

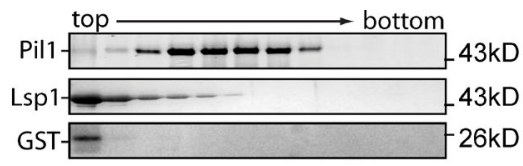

B

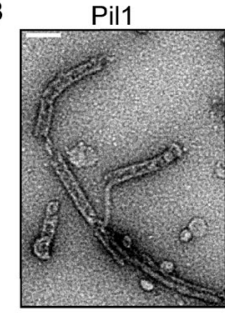

C
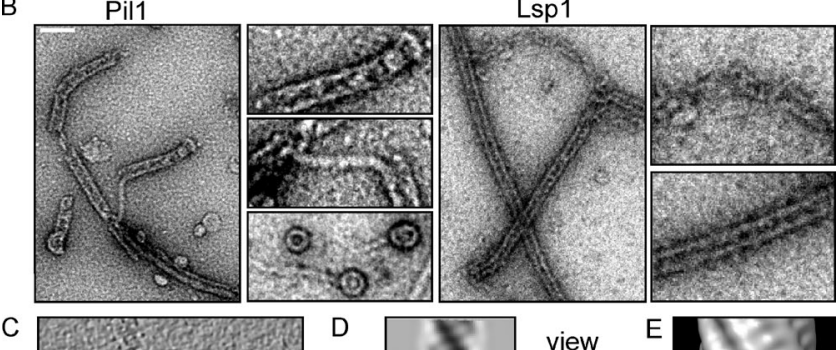

D
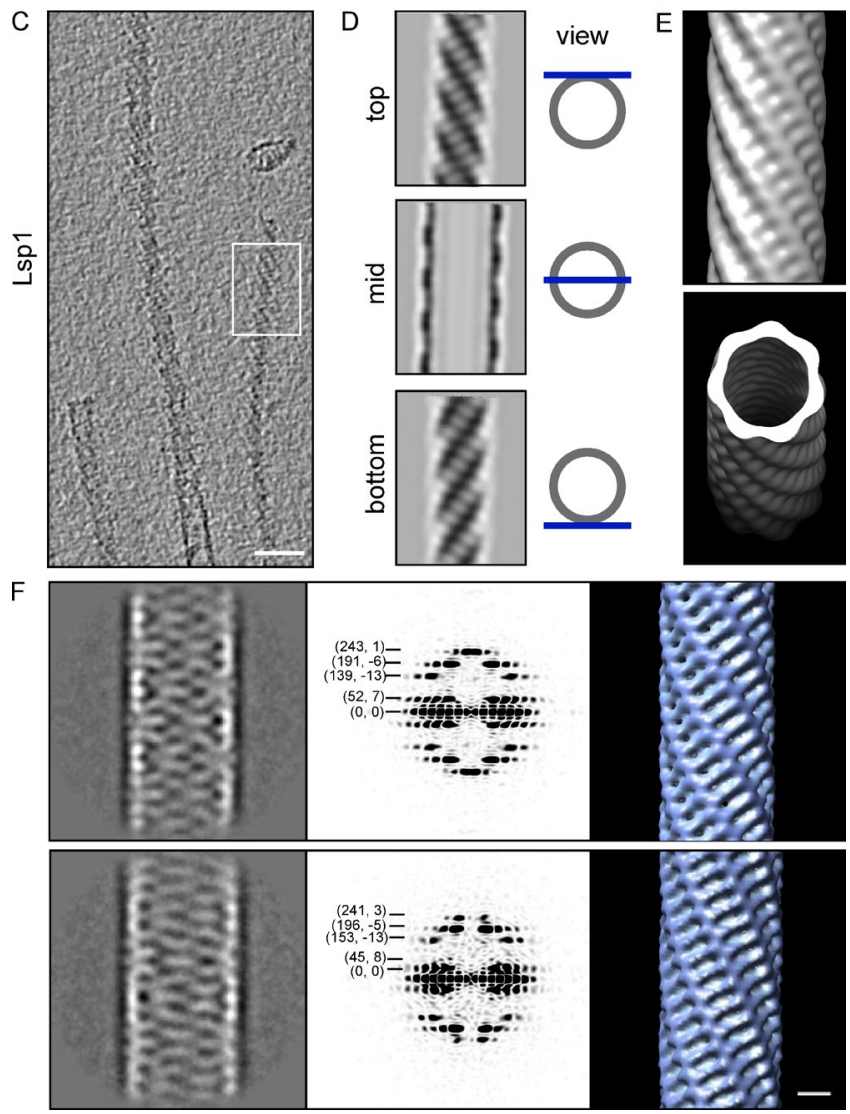

Figure 1. Pill and Lsp1 form filaments in vitro. (A) Pill and Lspl aggregate in vitro. SDS-PAGE of factions of a sedimentation velocity gradient analyzing recombinant Pill and Lsp 1. Protein marker sizes are indicated on the right. (B) Recombinant Pill and Lspl form filaments visualized by negative staining and EM. Pill assembles into ringlike structures as well as thin and thick filaments. Lsp 1 mostly forms thick filaments. Bar, $100 \mathrm{~nm}$. (C) Cryo-EM and tomographic reconstructions of Lspl filaments have a distinct striation pattern. Bar, $50 \mathrm{~nm}$. (D) Averaged tomographic top, mid, and bottom sections of a thick Lspl filament. (E) Surface rendering of the Lspl filament reconstruction. (F) Classification of Lspl segments reveal classes differing in diameter (left panels; the narrow class is shown on the top, whereas the wider class is shown on the bottom). Power spectra of both major classes are characteristic for filaments of helical symmetry (middle panels) and reveal differences in geometry, also visible in the resulting 3D maps (right panels). Bar, $10 \mathrm{~nm}$.

respectively; Ghaemmaghami et al., 2003; de Godoy et al., 2008). Recently, we discovered that the molecular structure of Pil1 and Lsp1s core part consists of a BAR domain (for Bin1, amphiphysin, and Rvs proteins; Ziółkowska et al., 2011). These banana-shaped domains are found across species in a

variety of proteins that commonly modulate membrane curvature in diverse processes, ranging from endocytosis to plasma membrane protrusion (Gallop and McMahon, 2005; Ren et al., 2006). In yeast, eisosome BAR domain proteins participate in membrane domain organization, as the normal plasma membrane domain pattern collapses and all fluorescently tagged MCC membrane proteins investigated so far mislocalize in pills cells, distributing uniformly over the membrane and forming one or a few large clusters, termed eisosome remnants (Walther et al., 2006; Grossmann et al., 2007; Fröhlich et al., 2009). In addition, pills cells have altered cellular signaling (Zhang et al., 2004) as well as endocytic rates of some, but not all, cargoes (Walther et al., 2006; Grossmann et al., 2008; Brach et al., 2011). Pill is not only required for normal plasma membrane distribution of proteins but also of lipids; in its absence, sterols distribute more evenly in the plasma membrane and accumulate at eisosome remnants (Grossmann et al., 2007). Thus, Pill provides an example of proteins that organize the plasma membrane in a highly tractable biological model system. In contrast, deletion of Lsp1 leads to only mild defects, but the molecular basis of the differences between these highly homologous proteins is unclear.

It is yet unknown how eisosomes are built, how they are targeted to the cell cortex, and how they organize the plasma membrane. To address these questions, we investigated the biochemical mechanisms of eisosome formation by Pil1 and Lsp1. Our study revealed a previously not recognized self-assembling scaffold that binds to and organizes the yeast plasma membrane.

\section{Results}

\section{Recombinant Pil1 and Lsp1 assemble in vitro}

Eisosomes are very large protein complexes containing primarily Pil1 and Lsp1. To test whether complex formation is mediated by autonomous assembly of Pil1 and Lsp1, we assayed the properties of the purified recombinant proteins by velocity sedimentation in a sucrose gradient. Both Pill and Lsp1 migrated into the gradient, albeit at different speeds. In contrast, soluble control proteins, such as GST, remained at the top of the gradient (Fig. 1 A). As Pill and Lsp1 themselves are relatively small $(\sim 38 \mathrm{kD})$, this result indicates that they assemble into large complexes.

To exclude the possibility that Pil1 and Lsp1 aggregate unspecifically, we investigated the structure of recombinant Pil1 and Lsp1 complexes by negative staining and EM. This analysis revealed intricate filamentous structures for both proteins, which are highly similar in sequence, but also some structural differences between their assemblies; Pil1 was present as a mixture of ringlike structures and two types of filaments, thin and thick ones (Fig. 1 B). Thick filaments appear to form by curling up thin filaments. In contrast, Lsp1 assemblies appeared structurally similar to thick Pill filaments but often ended into disordered chains. In Lsp1 samples, we rarely observed ringlike structures and never found thin filaments (Fig. 1 B, right). 
Pil1 and Lsp1 assemble already during purification of the proteins, and the resulting filaments likely form when the concentration of subunits reaches a critical threshold. Once formed, the assemblies could remain in dynamic equilibrium with free subunits, or, alternatively, they could represent stable complexes. To distinguish between these possibilities, we monitored exchange of recombinant Pill subunits between preassembled complexes. Complexes of Pill labeled with different fluorescent dyes appeared as foci in light microscopy images. A dynamic equilibrium between free subunits and the assemblies predicts that subunits exchange between red- or green-labeled preassembled complexes over time. However, even if we monitored the complexes' composition after $>3 \mathrm{~h}$, no exchange of labeled subunits occurred (Fig. S1 A). These data indicate that Pil1 assemblies are extremely stable in vitro, either as a result of high binding energy of the subunits to each other or as a result of a kinetic barrier for disassembly once a complex is formed.

We conclude that Pil1 and Lsp1 assemble into stable filamentous structures in vitro. Therefore, the formation of eisosomes is likely a consequence of intrinsic properties of Pil1 and Lsp1.

\section{Lsp1 filaments have helical symmetry}

To understand how Pil1 and Lsp1 filaments are built, we studied their structure in their native state by cryo-EM and 3D image reconstruction. We generated a structural model of Lsp1 filaments, as they are longer and much more ordered than analogous Pill structures, facilitating their analysis. Tomographic reconstructions of Lsp1 filaments display distinct striations (Fig. 1, C and D), and averaging of overlapping 3D segments along the axis of the filament shows grooves and ridges on the surface of a left-handed helix, corresponding to these surface features (Fig.1 E).

In a complementary approach, we used Fourier-Bessel analysis to reconstruct Lsp1 helices (DeRosier and Moore, 1970). Classification of segments revealed variation in the Lsp1 filaments, with at least two prominent classes, one being narrower and one being wider (Fig. $1 \mathrm{~F}$ and Table S3). Power spectra calculated for these two class averages show two very different helical symmetries, as manifested by differences in the position and Bessel order of the layer lines. For example, for the narrower filament (Fig. 1 F), the first layer line (52) has a Bessel order of 7, indicating the presence of a seven-start helix, whereas for the wider filament (Fig. 1 F), the first layer line (45) has a Bessel order of 8 , indicating the presence of an eight-start helix. $3 \mathrm{D}$ reconstructions calculated from class averages revealed a similar helical ordering of subunits (Fig. $1 \mathrm{~F}$ ) to that seen in the averaged structure from tomographic data (Fig. 1 E). As these two computational approaches were fully independent from each other, they cross-validate the derived structural models.

Pil1 and Lsp1 directly bind PIC4,5JPecontaining membranes

Pil1-GFP and Lsp1-GFP localization to the plasma membrane (Walther et al., 2006; Strádalová et al., 2009) could be mediated by direct binding to membranes, as observed for other BAR domain-containing proteins. To test this hypothesis, we incubated recombinant Pill or Lsp1 with liposomes mimicking the lipid composition of the plasma membrane and analyzed the resulting membrane structures by negative staining and EM. Fig. 2 A shows that both Pill and Lsp1 bind liposomes consisting of phosphatidylcholine (PC; $70 \mathrm{~mol} \%$ )/phosphatidylserine (PS; $15 \mathrm{~mol} \%$ ) and phosphatidylethanolamine (PE; $15 \mathrm{~mol} \%$ ) only in the presence of $1.5 \%$ phosphatidylinositol (PI)-4,5, bisphosphate $\left(\mathrm{PI}(4,5) \mathrm{P}_{2}\right)$ and deform them into long tubules.

To further test the lipid requirements for Pil1 and Lsp1 membrane binding, we incubated the proteins with liposomes made from PC, PC/PI, or PC/PI(4,5) $\mathrm{P}_{2}$. Even though we observed more abundant tubulation of $\mathrm{PI}(4,5) \mathrm{P}_{2}$ containing liposomes than of liposomes containing 1.5\% PI (Fig. 2 B), binding of Pil1 and Lsp1 is not strictly specific for $\mathrm{PI}(4,5) \mathrm{P}_{2}$.

To independently confirm Pil1 and Lsp1 membrane binding, we used a biochemical copurification assay. In floatation assays, protein complexes never migrated to the top of density gradients under conditions in which we observed membrane binding by EM. Therefore, we used cosedimentation of protein and liposomes, as they copelleted with membranes under such conditions. The interpretation of these experiments was further complicated by the self-assembly of Pill and Lsp1. Pil1 assemblies pelleted alone in the absence of liposomes, preventing their further analysis. In contrast, Lsp1 had a larger soluble pool ( $>50 \%$ of the protein under the conditions we used), and inclusion of $\mathrm{PI}(4,5) \mathrm{P}_{2}$-containing liposomes shifted all of Lsp1 to the liposome-containing bottom fraction, indicating membrane binding $(86 \pm 10 \%$; Fig. 2 C). In agreement with our observations by EM, membrane binding of Lsp1 was not completely $\mathrm{PI}(4,5) \mathrm{P}_{2}$ specific, but some binding also occurred in the presence of PI (Fig. 2 C) or PI(3P)-containing liposomes (not depicted).

As Pil1 and Lsp1 are highly homologous, we predict similar membrane-binding behavior for both proteins. As we could not analyze Pil1 by liposome cosedimentation, we used a different assay to test this hypothesis. To detect Pil1's membrane association, we coupled an environmentally sensitive NBD (4-nitrobenzo-2-oxa-1,3-diazole) fluorophore to a version of Pil1 containing a single cysteine residue at a position likely facing the membrane (S45C; based on the crystal structure; see Fig. 6 B). The resulting pil1S45C mutant localized normally, as determined by fluorescence microscopy in yeast cells expressing its GFP-tagged derivative (unpublished data). A hydrophobic environment (e.g., caused by membrane binding) increases the fluorescence emission of NBD compared with its fluorescence in aqueous solution. Consistent with membrane binding, NBDpil1S45C fluorescence increased 3.8-fold when incubated with liposomes containing $\mathrm{PI}(4,5) \mathrm{P}_{2}$ (Fig. $2 \mathrm{D}$, green emission spectrum) compared with the signal of the protein alone (Fig. 2 D, yellow emission spectrum). In agreement with our observations by EM, binding was not completely specific for $\mathrm{PI}(4,5) \mathrm{P}_{2}$, as we also observed a mild increase of NBD-pil1S45C fluorescence upon addition of PI-containing membranes (Fig. 2 D, purple emission spectrum). However, the increase in NBD-pil1S45C signal intensity induced by PI-containing liposomes was reduced in comparison with $\mathrm{PI}(4,5) \mathrm{P}_{2}$-containing liposomes (2.1-fold vs. 3.8-fold, respectively; Fig. 2 D). From these data, we conclude that Pil1 and Lsp1 directly bind membranes, preferably those containing $\mathrm{PI}(4,5) \mathrm{P}_{2}$. 
Figure 2. Pill and Lspl directly bind $P I(4,5) P_{2}$-containing membranes. (A) Pill and Lsp 1 bind and tubulate $\mathrm{PI}(4,5) \mathrm{P}_{2-}^{-}$ recombinant Pill or Lspl incubated with liposomes con$1.5 \% \mathrm{PI}(4,5) \mathrm{P}_{2}$. (B) Negative-stained samples of recombinant Pill or Lspl incubated with PC liposomes containing $1.5 \% \mathrm{PI}$ or $\mathrm{PI}(4,5) \mathrm{P}_{2}$. Insets show magnifications of Pill bound to liposomes. (A and B) Protein-covered membrane tubules are marked with yellow arrowheads. Bars, $100 \mathrm{~nm}$. (C, top) Spin-down experiments of Lspl incuor $\mathrm{PI}(4,5) \mathrm{P}_{2}$ as indicated. Proteins bound to liposomes appear in the pellet (P). Lspl shows higher affinity to $\mathrm{PI}(4,5) \mathrm{P}_{2}$ than to PI. S, supernatant. (bottom) Quantification of protein amounts in the pellet fractions from spindown experiments represented in a box plot, consisting of the median (middle of the box), the upper and lower quartile (edges of the box), and whiskers at a 1.5-interquartile range distance from the upper and lower quartile. (D) Measurement of fluorescence from NBD-labeled pil 1S45C (orange emission spectrum) alone as well as in the presence of PC/PS/PI liposomes (purple emission spectrum) or $\mathrm{PC} / \mathrm{PS} / \mathrm{PI}(4,5) \mathrm{P}_{2}$ (green emission spectrum); the buffer control is shown in gray. $n=6$. containing liposomes. Negative staining and EM of taining $\mathrm{PC} / \mathrm{PS} / \mathrm{PE}(70 \% / 15 \% / 15 \%)$ or, in addition, bated with or without PC liposomes containing $1 \% \mathrm{PI}$

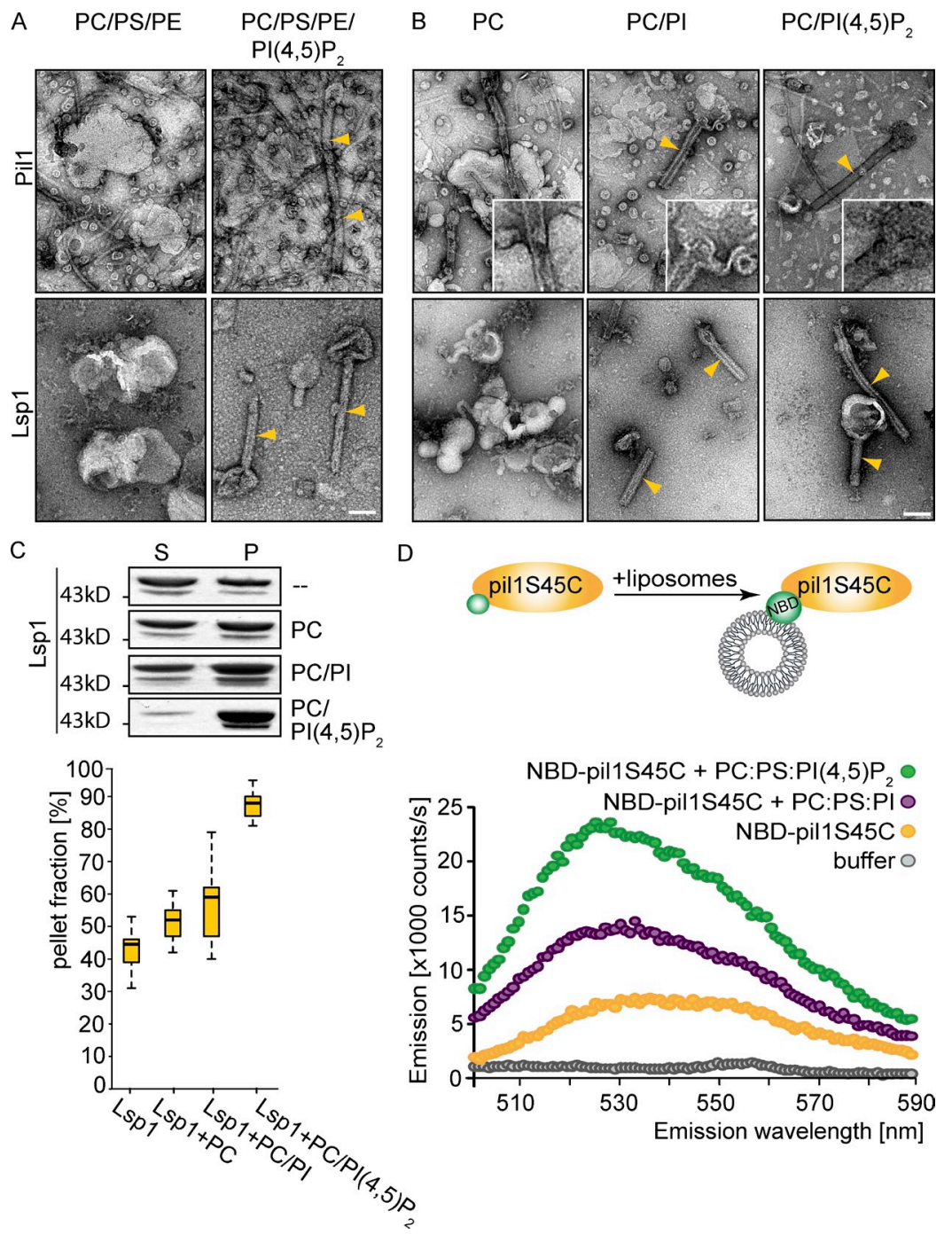

PIC4,5]P2 is required for the formation and organization of eisosomes in vivo

As Pil1 and Lsp1 preferentially bind $\mathrm{PI}(4,5) \mathrm{P}_{2}$ in vitro, a reduction of the plasma membrane-specific $\mathrm{PI}(4,5) \mathrm{P}_{2}$ pool may lead to impaired eisosome localization in vivo. To deplete $\mathrm{PI}(4,5) \mathrm{P}_{2}$ from the plasma membrane and to test consequences on eisosomes, we used a yeast mutant containing a temperature-sensitive allele of MSS4 ( $\mathrm{mss}^{\mathrm{ts}}$ ), encoding the kinase that converts PI-4phosphate (PI4P) to $\mathrm{PI}(4,5) \mathrm{P}_{2}$. Inactivation of Mss4 after a temperature shift results in the depletion of $\mathrm{PI}(4,5) \mathrm{P}_{2}$ from the plasma membrane (Stefan et al., 2002). Under such conditions of reduced $\mathrm{PI}(4,5) \mathrm{P}_{2}$ levels, no Pill-GFP signal remained in an eisosome pattern in $m s s 4^{t s}$ cells, but, instead, all of the protein clustered into enlarged structures at the membrane or became cytosolic (Fig. 3 A). Time-lapse imaging of eisosomes, marked by Pil1-GFP, suggests that eisosomes progressively detach from the plasma membrane and aggregate under these conditions (Videos 1-3). This was specific for cells containing the $m s s 4^{t s}$ allele, as we did not observe a similar phenotype in control cells expressing Pil1-GFP. Depletion of other phosphoinositides, such as PI4P at the Golgi apparatus in temperature-sensitive pikl mutants and PI3P in vps $34 \Delta$ cells, did not lead to a comparable phenotype (Fig. S1, D and E). We also found that stt4 mutants impaired in generating PI4P at the plasma membrane and thus indirectly impaired in $\mathrm{PI}(4,5) \mathrm{P}_{2}$ synthesis displayed abnormal Pill organization, albeit much weaker than mss 4 cells (Fig. S1 D). Moreover, $\mathrm{PI}(4,5) \mathrm{P}_{2}$ is important for normal plasma membrane domain organization, as $\mathrm{PI}(4,5) \mathrm{P}_{2}$ depletion results in loss of the punctate Sur7 localization, an MCC domain marker, which was instead more evenly distributed over the plasma membrane of $m s s 4^{t s}$ cells after the temperature shift (Fig. 3 B).

In addition, increase of $\mathrm{PI}(4,5) \mathrm{P}_{2}$ in the plasma membrane had the opposite effect as its decrease; deletion of two $\mathrm{PI}(4,5) \mathrm{P}_{2}$ phosphatases encoded by the yeast synaptojanin-like proteins (SJL1 and SJL2) leads to increased $\mathrm{PI}(4,5) \mathrm{P}_{2}$ levels (Stefan et al., 2002) and Pil1-GFP assembly into much larger structures that appear to protrude from the plasma membrane into the cytoplasm (Fig. $3 \mathrm{C}$ ). Thus, Pil1 interaction with $\mathrm{PI}(4,5) \mathrm{P}_{2}$ is crucial for normal eisosome formation and plasma membrane domain organization.

To further test the physiological significance of eisosome protein interaction with $\mathrm{PI}(4,5) \mathrm{P}_{2}$, we tested genetic interactions between PILI and SJL1 in epistatic miniarray profiles (E-MAPs; (Collins et al., 2006)). E-MAPs contain quantitative measurements of genetic interactions within a selected set of mutants. 
A
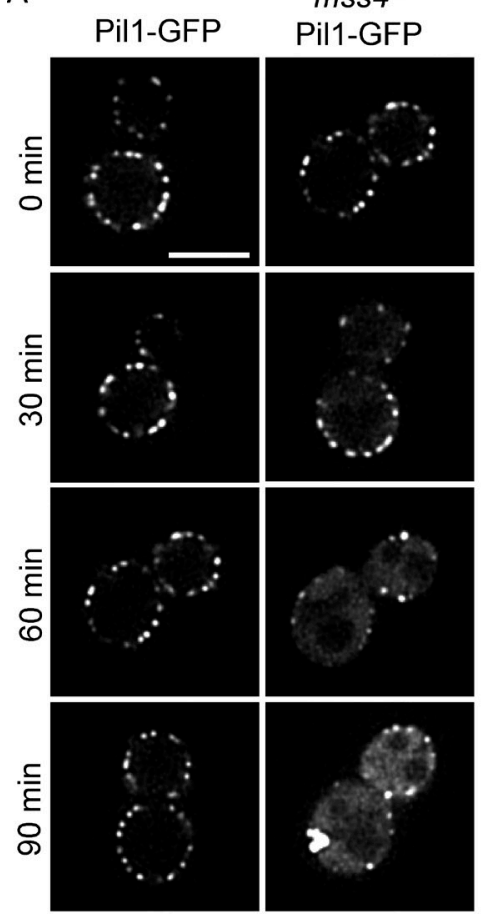

C

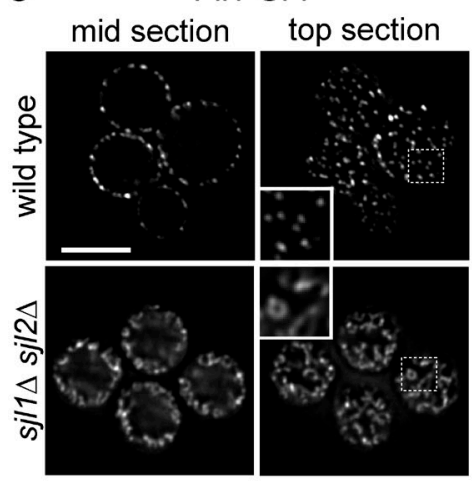

B $m s s 4^{t s}$ Pil1-GFP
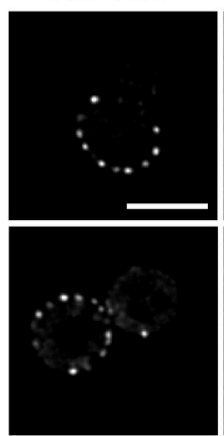

.
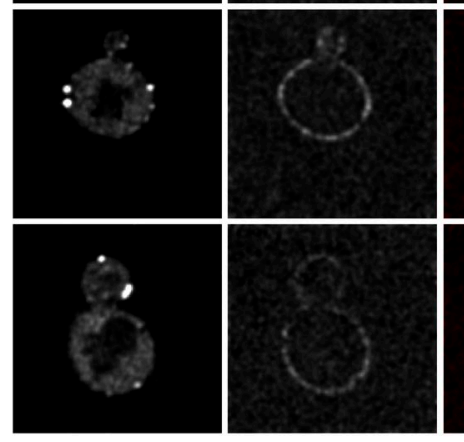

D

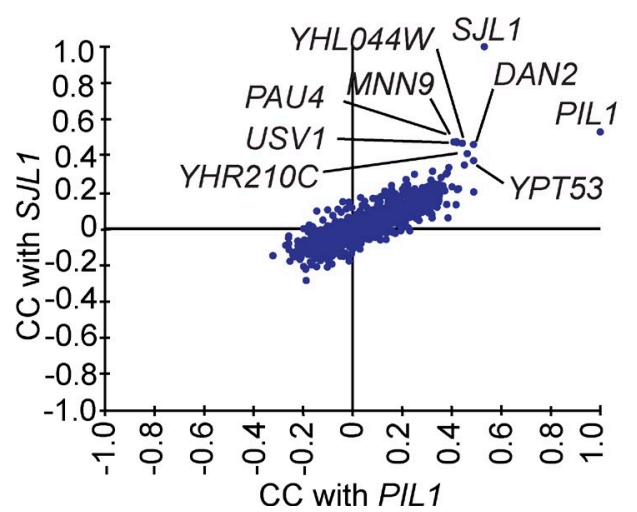

Figure 3. $\quad \mathbf{P I}(4,5) \mathbf{P}_{2}$ is necessary for normal eisosomes in vivo, and PIL1 has a highly similar genetic profile to SJL1. (A) Fluorescence microscopy of Pil 1-GFP in a yeast mutant strain containing a temperature-sensitive allele of MSS4 (mss4 $\left.4^{\text {ts}}\right)$. Pill-GFP loses its normal eisosome pattern but instead clusters to enlarged structures at the membrane after a 90-min (right column) temperature shift from 24 to $37^{\circ} \mathrm{C}$. The control strain does not show this phenotype (left column). (B) Fluorescence microscopy of Sur7-mars and Pill-GFP in mss4 $4^{\text {ts }}$ cells. After $30 \mathrm{~min}$ of temperature shift, Sur7mars loses its localization to the MCC. After $60 \mathrm{~min}$, it is evenly distributed in the plasma membrane. (C) Deletion of SJL1 and SJL2 results in increased Pill-GFP assembly at the plasma membrane. Insets show magnified regions of cells in the boxed areas. Bars, $5 \mu \mathrm{m}$. (D) Comparison of correlation scores from an E-MAP focusing on lipid metabolism. SJL1, encoding the $\mathrm{Pl}(4,5) \mathrm{P}_{2}$ phosphatase, has the most similar genetic signature to PIL 1, indicating similar gene function. CC, correlation of correlations.
If two mutations have similar physiological consequences, they will share many suppressing or aggravating genetic interactions with other mutations, resulting in highly similar genetic interaction profiles. Strikingly, the genetic profiles of PIL1 and SJL1 are most similar to each other in two independently generated E-MAP datasets. For example, within an E-MAP containing 787 genes mainly functioning in lipid metabolism, PILI and SJL1 interactions with all other genes were more similar to each other than any other gene in the set (Fig. $3 \mathrm{D}$, correlation of genetic profiles of PILI and SJL1 $=0.8281$ and 0.332 in an E-MAP focusing on plasma membrane function; Fig. S1 F; Aguilar et al., 2010), showing that deletions of PILI or SJL1 have very similar phenotypic consequences. These findings further argue that $\mathrm{PI}(4,5) \mathrm{P}_{2}$ interaction of eisosome components is physiologically important.

\section{Structure of membrane-bound Pil1 and Lsp 1}

Having established that eisosome proteins Pill and Lsp1 directly interact with $\mathrm{PI}(4,5) \mathrm{P}_{2}$-containing membranes, we determined the structure of the resulting assemblies. To this end, we used a combination of cryo-EM, tomography, and 3D image reconstruction. Computational slices through 3D tomograms revealed that both Pil1 and Lsp1 decorate liposomal membranes exclusively from the outside, thereby constricting them to long tubes with a similar diameter as the helices formed by the protein alone (Fig. 4 A). Interestingly, the diameter of Pil1 or Lsp1decorated tubules was always smaller than the diameter of the membrane tubule emerging from the end of the protein filaments, suggesting that the proteins bend membranes, thereby constricting them to a smaller diameter (Fig. 4 A). In addition, Pil1 especially formed much longer filaments on liposomes compared with the rather short and unordered filaments in the absence of membranes, indicating a possible role of proteinmembrane interaction in assembly.

Further classification analysis of filament segments from cryo-EM revealed variation in the diameter of the tubules, which was most pronounced for Pill bound to membranes ( 30-37 nm). Also, tubules formed by Lsp1 bound to membranes 
Figure 4. Structure of membrane-bound Pill and Lsp1. (A) Structure of Lspl and Pill bound to $\mathrm{PI}(4,5) \mathrm{P}_{2}$-containing liposomes. Tomographic midsections show that both proteins decorate liposomes and constrict them to a similar diameter. Bar, $50 \mathrm{~nm}$. (B) Classification of Lspl filament segments in addition to membrane-bound Lspl and Pil 1 reveals different diameters. $\mathrm{N}$ represents the number of segments used for the classification. (C) Helical reconstruction of prominent groups of Lspl filaments as well as membranebound Lspl and Pil1. Membrane-bound Lspl shows distinct density oriented toward the lipid bilayer. Bar, $10 \mathrm{~nm}$.
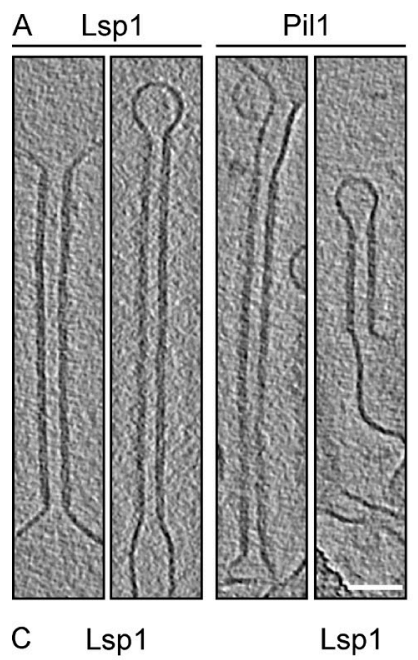
\#1 30nm
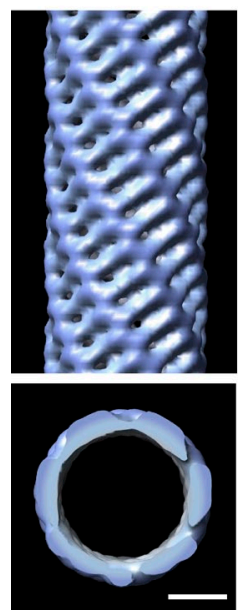

B

$$
\begin{array}{lll}
\text { Lsp1 } & \text { Lsp1 } & \text { Pil1 }+ \\
\text { N=18,628 } & \text { liposomes } & \text { liposomes } \\
& N=4,212 & N=13,565
\end{array}
$$
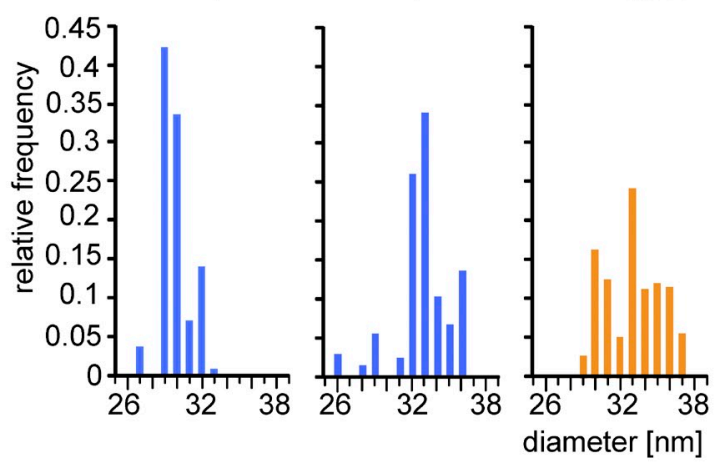

Lsp1+liposomes Pil1+liposomes \#1 34nm \#1 34nm

Pil1+liposomes \#3 36nm

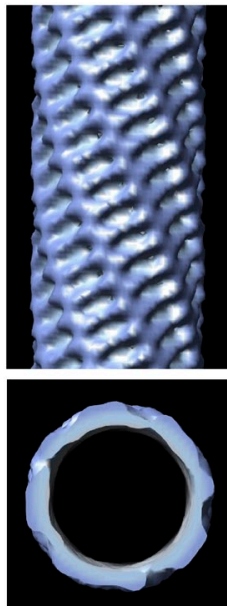

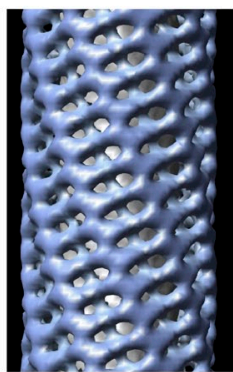
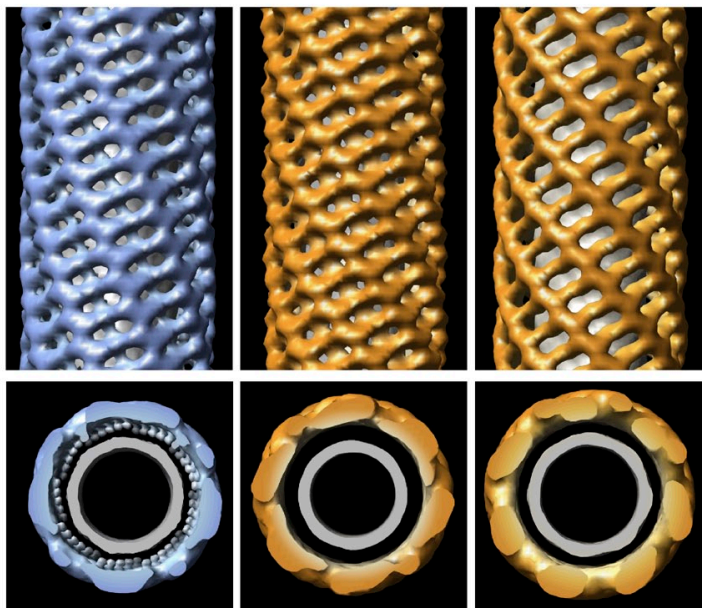

showed such diameter variation $(\sim 32-36 \mathrm{~nm})$, and the diameter of the protein alone was always smaller than in its membranebound state ( $\sim 29-32 \mathrm{~nm}$; Fig. 4 B).

To gain more detailed information on the subunit arrangement in the thick filaments, we used a combination of two helical reconstruction approaches. 3D reconstructions from Fourier-Bessel analysis provided different low-resolution structures for supervised classification of all segments into respective symmetry classes and for refinement of the $3 \mathrm{D}$ reconstruction using iterative helical real-space reconstruction (IHRSR). This method is especially suited for flexible filaments with helical symmetry (Egelman, 2007). All structures showed a repeating unit with similar morphology and local twofold symmetry, consistent with their assembly from BAR domain dimers. However, the helical arrangement of subunits varied in the different structures (Fig. $4 \mathrm{C}$ and Table S3). Notably, the repeating structural units in Lsp1 and Pil1 filaments closely resemble each other, consistent with the high primary sequence similarity between the two proteins (Fig. 4 C). In each of the filaments, subunits form a fenestrated protein coat around the liposome membrane, potentially allowing access of small molecules and proteins to the membrane. In the Lsp1 structure, additional density was visible in the model derived for membranebound protein compared with the one of the protein alone
(Lsp1 + liposomes \#1; Fig. S1 B). We believe that this density represents a part of Lsp1 that gains higher order upon membrane binding and thus becomes detectable.

Variation in the filament diameter and helical symmetry argues for high flexibility in these assemblies. Thus, we addressed whether the helical symmetry can change within a single filament. As only two main classes, narrow $(30 \mathrm{~nm})$ and wide $(32 \mathrm{~nm})$, were detected for Lsp1 filaments in the absence of the membrane (Fig. 4 C, Lsp1 \#1 and \#2), we used these data in our analysis for simplicity. The distribution of the two classes along the filament was not random. On the contrary, the probability for a narrow segment succeeding a narrow segment was 0.97 . Conversely, the probability for a wide segment succeeding a wide segment was 0.89 . This finding not only reflects filament architecture but also indicates that our analysis is sensitive enough to detect subtle differences. Continuous stretches of narrow or wide segments were often present in a single filament (Fig. S1 B). Extrapolation of these results for Lsp1 filaments in the absence of the membrane to Lsp1 and Pill filaments in the presence of the membrane indicates that the latter may have even more complicated mixtures of different helical symmetries.

Although variable, the diameter of the underlying membrane tubule correlated with the diameter of the outer protein coat. 


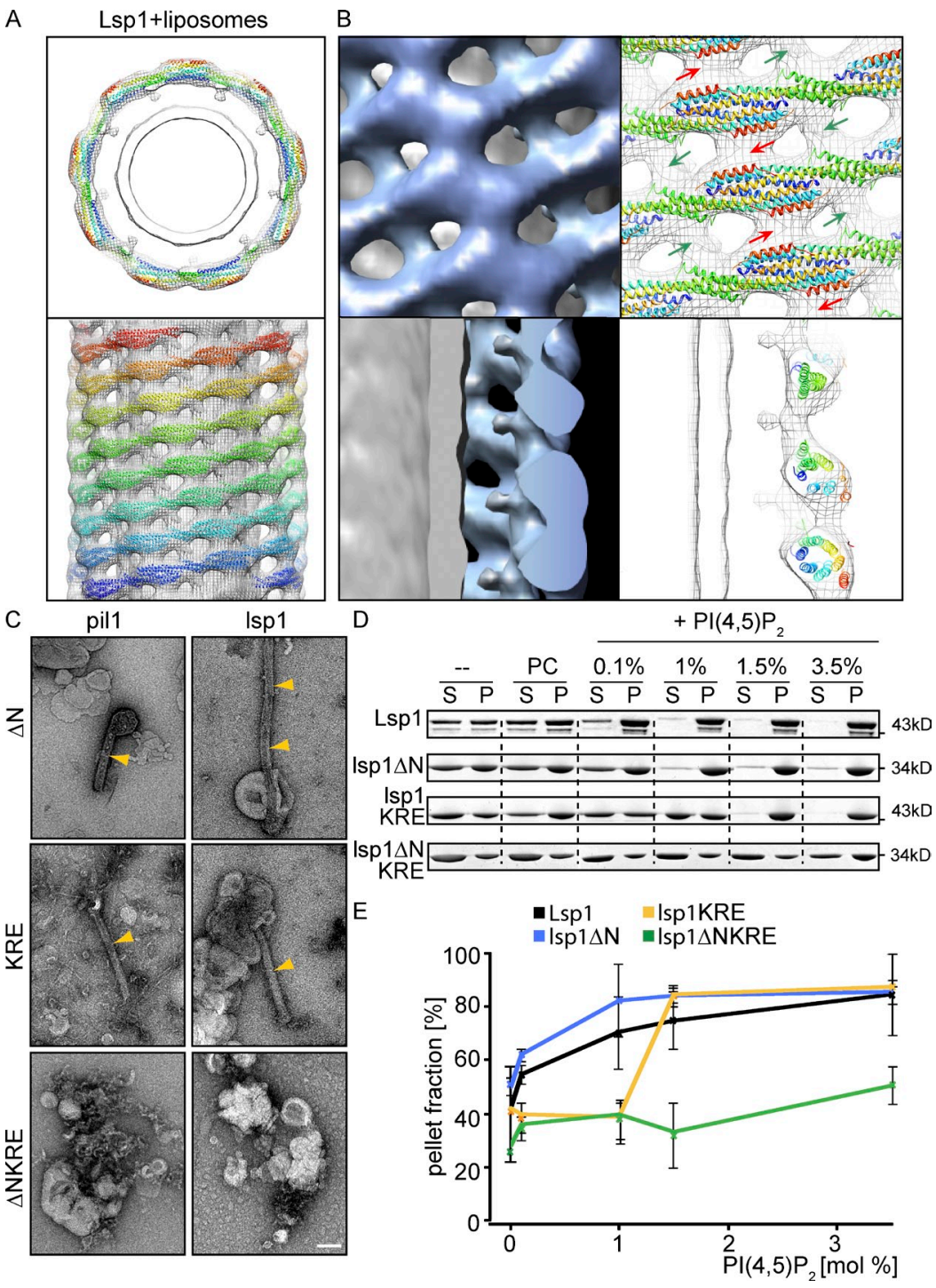

Figure 5. Pill and Lspl membrane binding requires an $\mathrm{N}$-terminal segment and a patch of positively charged amino acids on their BAR domain surface. (A) Computational rigid body fitting of the Lspl BAR domain dimer $x$-ray structure to cryo-EM density maps of Lsp 1 tubules and Lspl bound to $\mathrm{PC}$ liposomes containing $1.5 \% \mathrm{PI}(4,5) \mathrm{P}_{2}$. The top view of tubules (top) shows Lspl BAR domain monomer chains colored blue to red from $\mathrm{N}$ terminus to $\mathrm{C}$ terminus. The side view of tubules (bottom) shows the Lsp 1 helix colored blue to red from the bottom to the top. (B) A close-up of the side view and intersection of the tubules. Lspl BAR domain monomer chains are colored blue to red from the $N$ terminus to the $C$ terminus. Density that might be occupied by the flexible tips of the x-ray structure, adopting a slightly different conformation in the tubules than in the crystal, is indicated by green arrows. The density that could be filled by the $C$ termini, which are missing in the $x$-ray structure, is indicated by red arrows. (C) Negative staining and EM of recombinant Pil 1 or Lspl proteins incubated with PC liposomes containing either 1.5 or $3.5 \% \mathrm{PI}(4,5) \mathrm{P}_{2}$. Mutants with an $\mathrm{N}$-terminal truncation $(\mathrm{lspl} \Delta \mathrm{N})$ or changes in the positively charged amino acid patch of the concave BAR domain surface of Pill or Lspl (Isp1KRE) retain the ability to bind and tubulate $\mathrm{PI}(4,5) \mathrm{P}_{2}$-containing liposomes. Combination of both types of mutation (lspl $\triangle$ NKRE) abolishes membrane binding. Protein-covered membrane tubules are marked with yellow arrowheads. Bar, $100 \mathrm{~nm}$. (D) Spin-down experiments of Lspl, IsplKRE, Ispl $\Delta \mathrm{N}$, or Ispl $\Delta$ NKRE incubated with or without PC liposomes containing 0.1, $1,1.5$, or $3.5 \% \mathrm{PI}(4,5) \mathrm{P}_{2}$ as indicated. Panels showing different experimental conditions are separated by dotted lines for better visibility. P, pellet; S, supernatant. (E) Quantification of protein amounts in pellet fractions of experiments analogous to D. $n=3$. Error bars represent SDs of three independent experiments.
The consistent distance between visible membrane and proteins observed in different classes and samples argues for a specific protein-membrane interaction that imposes a specific curvature on the membrane tubule.

\section{Pil1 and Lsp1 membrane binding requires an $\mathbf{N}$-terminal protein segment and a patch of positively charged amino acids in their concave BAR domain surface}

Recently, we discovered by crystallographic analysis of Lsp1 that eisosome proteins contain BAR domains (Ziółkowska et al., 2011). To investigate how Lsp1 BAR domains assemble into helical filaments and interact with the membrane, we computationally fitted models of Lsp1 dimers into our cryo-EM maps. Specifically, we used six different EM-derived density maps of assembled Lsp1 and Pil1 to fit three variations of the structural model of Lsp1 amino acids 51-264 varying in the conformation of the BAR domain tips (based on the comparison of AA, BB, and $\mathrm{CC}$ dimers, constructed by superimposition of $\mathrm{A}, \mathrm{B}$, and $\mathrm{C}$ chains present in the asymmetric unit on the crystallographic dimer AB; Ziółkowska et al., 2011). Fitting always placed the dimers at the same relative position in the cryo-EM map and always resulted in the same orientation of BAR domains with their concave side facing the membrane surface (Fig. 5 [A and B] and not depicted). For each map, the BB dimer gave slightly higher scores than AA and CC, most likely because its structure lacks a loop sequence and thus has a slightly smaller volume. To generate atomic models of the filaments, we imposed helical symmetry parameters of the cryo-EM density maps on the best-fitting dimers. This revealed clashes between the tips of the BAR domain dimer and its symmetry-related neighbors, in addition to densities, which were not occupied by the fitted chains (Fig. 5 B). We consider two explanations for these discrepancies. First, superimposition of AA, BB, and CC dimers suggests that the tips are the most flexible part of the Lsp1 BAR domain (Ziółkowska et al., 2011). Therefore, it is possible that the dimers change conformation in the assembled helices compared with the crystal structure and move their tips into the density that is located right next to the clashing region and which is not filled in our fitting (Fig. 5 B, green arrows). Second, some of the unaccounted density could be occupied by the Lsp1 $\mathrm{N}$ or C terminus, which are absent in the x-ray structure (Fig. 5 B, red arrows). 
Figure 6. Purified eisosome proteins from yeast structurally resemble recombinant Pill or Lsp1 protein assemblies. (A) Tandem affinity chromatography of tagged Pill enriches mainly Pil1, Lspl, and Mrp8. Negative staining and EM reveal highly similar structures for purified eisosomes (right) as formed by recombinant Pill (left). Side panels show Coomassie blue-stained SDS-PAGE gels of the preparations used. (B) X-ray structure of dimeric Lspl BAR domain. Monomers are shown as a ribbon representation in green and gray. Residues that can be phosphorylated and that are represented in the structure of eisosome protein BAR domains are highlighted in red. (C) Purification of recombinant pil 14D) and visualization by negative staining and EM show that pil 1 (4D) does not form thick helices but only long, thin filaments. Bars, $100 \mathrm{~nm}$. (D) Precipitated fractions of sedimentation velocity gradients of recombinant Pil 1 and pil 1(4D) were analyzed by SDS-PAGE. They show different mobility of phosphorylation mutants compared with wild-type Pill.
A

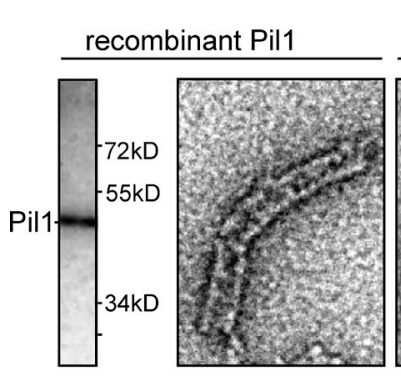

S.cerevisiae Pil1
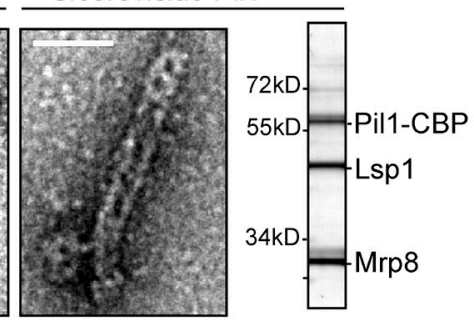

C

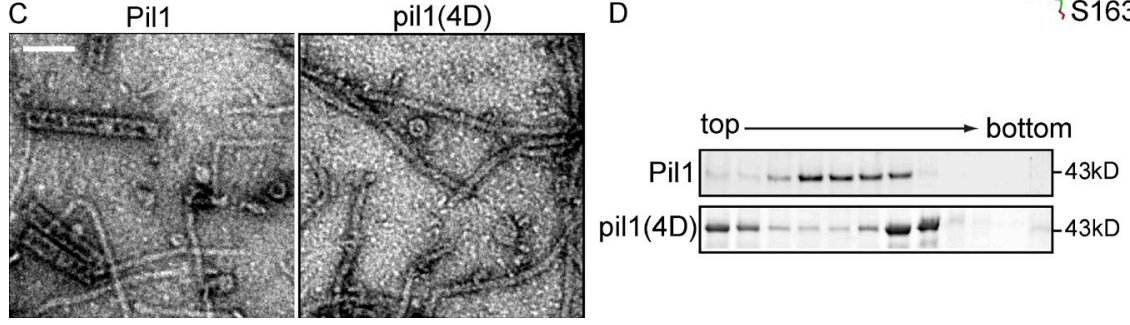

B

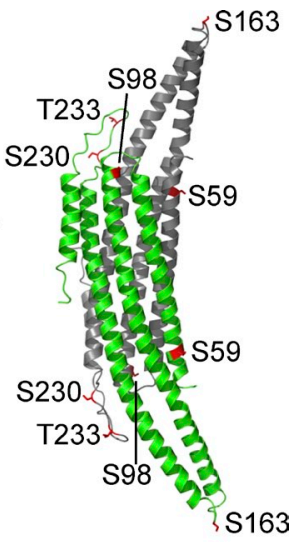

These structural data show that Lsp1-and by extension, the almost identical Pil1 (Ziółkowska et al., 2011)—assembles by interactions at the end of the banana-shaped dimers with the concave surface facing the membrane, similar to other BAR domain proteins (Frost et al., 2008).

To test our model of Lsp1 assembly on membranes and to determine which regions of the protein are required for membrane interaction, we tested various mutants of Pil1 and Lsp1. Particularly, a patch of positively charged amino acids on the concave surface of the Lsp1 BAR domain is evolutionary conserved and required for normal localization of the proteins in vivo (Ziółkowska et al., 2011). However, mutation of two positively charged amino acids in this patch to glutamic acid (pil1KRE and lsp1KRE, carrying K130E and R133E mutations) did not completely abolish membrane binding, as determined by negative staining and EM with liposomes (Fig. 5 C). Similar results were obtained in spin-down experiments, which revealed reduced, but not abolished, membrane binding of 1sp1KRE (Fig. 5, D and E). Intriguingly, in our structural model (Fig. 4 C), we observed one small protrusion per dimer of full-length Lsp1 proteins toward the membrane surface that were not filled by the crystal structure representing the BAR domain alone (missing the $\mathrm{N}$ terminus). Moreover, the most $\mathrm{N}$-terminal segment of the crystal structure is oriented toward the membrane. Therefore, we hypothesized that, in analogy to membrane binding of other BAR domains, protein segments N-terminal of the BAR domain of two proteins together mediate membrane interaction. To test this model, we deleted the N-terminal 35 amino acids (pil1 $\Delta \mathrm{N}$ and $1 \operatorname{sp} 1 \Delta \mathrm{N}$ ). Intriguingly, when we analyzed these mutants in the absence of liposomes, we found only thin, but no thick, helices (Fig. S1 C). In the presence of liposomes, we observed thick helices and no defect in membrane binding by $\mathrm{EM}$ analysis or in spin-down experiments for pill $1 \Delta \mathrm{N}$ or $1 \mathrm{sp} 1 \Delta \mathrm{N}$ (Fig. 5, C-E), indicating that the BAR domain alone is sufficient for membrane binding. Importantly, when we mutated the positive patch in the context of a mutant protein that lacks the
$\mathrm{N}$ terminus (pil1/lsp1 $\Delta \mathrm{NKRE}$ ), membrane binding of Pil1 or Lsp1 was completely abolished (Fig. 5, C-E). These data show that both the $\mathrm{N}$ terminus and a patch of positively charged residues on the concave surface of the BAR domains of Pill and Lsp1 are required for their interaction with membranes.

\section{Purified eisosomes from yeast resemble recombinant Pil1 and Lsp1 helices}

Next, we analyzed whether the structures of Pil1 and Lsp1 determined using in vitro methods reflect the arrangement of the proteins on eisosome membrane furrows observed in vivo (Strádalová et al., 2009). To this end, we first purified Pill fused to a tandem affinity purification tag by affinity chromatography together with associated eisosomes proteins from yeast cells. These experiments yielded a complex consisting of Pill (with a CaM-binding peptide remaining on the protein after elution by cleavage of the tag), Lsp1, and Mrp8 (Fig. 6 A; Walther et al., 2006; Wang et al., 2009). Mrp8 is a cytoplasmic protein, which associates with Pil1 and Lsp1 but does not play a structural role at eisosomes (Fig. S2). To test whether purified eisosome proteins from yeast assemble into structures similar to those formed by the recombinant proteins, we performed negative staining and EM of these preparations and found structures that resembled each other in overall shape and dimensions (Fig. 6 A). This indicates that the structural information derived from the recombinant proteins likely reflects the organization of eisosomes in cells.

\section{Phosphomimicking mutations of Pil1 block formation of thick helices in vitro}

To further test whether the arrangement of Pil1 and Lsp1 is physiologically relevant, we tested the prediction that their assembly should change in vitro under conditions that change eisosomes in vivo. Pil1 is phosphorylated on several residues in vivo, and alterations in its phosphorylation state affect eisosome assembly in yeast (Walther et al., 2007; Luo et al., 2008). In a mutated form of Pil1, denoted pil1(4D), four normally 
phosphorylated residues were changed to phosphomimicking aspartates (S45D, S59D, S230D, and T233D; Fig. 6 B). Pil1(4D) does not mimic all known phosphorylations of Pil1, but its four mutations are necessary and sufficient for mediating the Pill phosphorylation effect that results in just one or a few large clusters and a much stronger cytoplasmic signal compared with wild-type Pil1 (Walther et al., 2007). To investigate whether this effect is mediated by altering Pill's self-assembly, we first analyzed recombinant pil1(4D) by velocity gradient centrifugation and found that sedimentation of the mutant protein was significantly altered compared to the wild-type form (Fig. 6 D). Specifically, a large pool of the mutated protein remained at the top of the gradient, and, in addition, a faster-migrating species increased in abundance.

To visualize the effect of phosphomimicking mutations on Pil1 self-assembly, we analyzed the structure of pil1(4D) by negative staining and EM. These experiments showed that pil1(4D) is still able to assemble into thin filaments, which were abundantly present in the sample. However, we never observed thick helices that are common in wild-type Pill samples (Fig. 6 C). This indicates that the phosphomimicking mutations and, by extrapolation, phosphorylation of Pill lead to impairment of helical assembly. The analogous consequences of Pill phosphomimicking mutations observed in vivo and in vitro provide additional support for the interpretation that Pil1 and Lsp1 helices are equivalent to eisosomes.

Eisosomes form short helical lattices at the plasma membrane in yeast cells

To further test whether Pil1 and Lsp1 helices observed in vitro resemble eisosomes in vivo, we investigated their structure in yeast cells. Consistent with the data of Strádalová et al. (2009), freeze-fracture deep-etching EM (DEEM) analysis of the yeast plasma membrane showed abundant furrows, whose presence depended on PIL1 (not depicted; Strádalová et al., 2009). Previously, it was shown by immunogold labeling that these furrows contain Pill (Strádalová et al., 2009). Our images of the furrows revealed distinct striations resembling the surface pattern of Pil1 and Lsp1 assemblies formed in vitro (Fig. S3 A). However, as freeze-fracture DEEM images show the interface between the two lipid layers of the plasma membrane (P-face), eisosome proteins are not directly visible in such images, as they are still covered by a lipid monolayer. To overcome this limitation, we bound yeast cells to a grid as a solid support and removed most of the cells by a short burst of ultrasound waves (Fig. S3 D; Hanson et al., 2008). Notably, this technique requires spheroblasting of yeast cells, which we found can lead from mild to severe elongation of eisosomes, as visualized by fluorescent microscopy. Therefore, we developed a mild spheroplasting protocol, with only minor effects on the fluorescent signal of eisosomes (Fig. S3 C). This technique allowed us to image the cytoplasmic face of the plasma membrane that remains bound to the grid by DEEM, which exposed abundant filamentous structures that we suspected to be eisosomes (Fig. 7 A). To test this hypothesis, we labeled Pill or Lsp1 either with an affinity-purified polyclonal antibody against full-length Pill or against GFP in cells that express tagged versions of Pill or
Lsp1 (Figs. 7 B and S3 B). Each of the antibodies specifically labeled the filamentous structures, thereby confirming the presence of Pil1 and Lsp1 within them. Importantly, the filaments showed striations characteristic for Pil1 and Lsp1 assemblies in vitro (Fig. 7 A). However, in contrast to the helical structures observed in vitro, eisosomes consist of half-cylinders that cover the cytoplasmic face of furrows, which can be best seen in 3D anaglyphs of DEEM images, representing the cytoplasmic side of the plasma membrane or the P-face (Fig. 7, C and D). In some, but not all, cases, these helical structures resided at the side of large membrane invaginations (Fig. 7, A and B, arrowheads). Collectively, these data show that eisosomes resemble the structure of Pil1 and Lsp1 assemblies determined in vitro.

\section{Discussion}

Eisosome proteins play an important structural role in organizing the yeast plasma membrane. As expected from their architectural function, eisosome proteins localize very stably and are extremely high in abundance. For comparison, copy numbers of main eisosome components per cell $(115,000$ for Pill and 104,000 for Lsp1) are much larger than those of tubulin $(5,590)$ or actin (60,000; Norbeck and Blomberg, 1997; Ghaemmaghami et al., 2003). As a result of these properties, Pil1 and Lsp1 could be classified as part of a membrane cytoskeleton. Consistent with this idea, we show that Pill and Lsp1 self-assemble into a protein scaffold that binds and deforms membranes, providing a mechanism for how these proteins organize the plasma membrane into domains.

Similar to other BAR domain-containing proteins (such as F-BARs; Frost et al., 2008), Pil1 and Lsp1 form higher-order complexes on membranes. In contrast to other BAR domain proteins, however, the assemblies formed by eisosome proteins are extremely stable in the absence of membranes. Even though we cannot pinpoint exact contacts as a result of limited resolution, our current model of eisosome architecture posits three distinct interactions: one to form the BAR dimer (interaction 1), a second end-to-end contact of BAR domain dimers to form thin filaments (interaction 2), and a third lateral interaction to form helices (interaction 3; Fig. 8). The overall similarity and subtle differences of the assemblies formed by Pill and Lsp1 suggest that the strength of the different interactions may differ between the two proteins. For example, Lsp1 interaction 2 may have a lower affinity compared with Pil1, which would result in a requirement for combined binding energy from endto-end interactions 2 and lateral interactions 3 to stabilize thick Lsp1 helices. Smaller assemblies, such as thin filaments, may be unstable and fall apart, explaining the absence of thin Lsp1 filaments and the increased pool of nonassembled Lsp1 in cells and biochemical assays. Immunolocalization of Pil1 and Lsp1 in EMs performed in this study (and Strádalová et al. [2009]) shows that both proteins are present in eisosomes covering membrane furrows, but how both proteins associate to form them and whether their different properties are used to modulate eisosome structure are yet unclear.

Interaction 3 is likely modulated by phosphorylation, as indicated by its sensitivity to phosphomimicking mutations of 
Figure 7. Eisosomes in situ structurally resemble Pill and Lspl assemblies. (A) Representative image of the yeast plasma membrane from the cytosolic side (top). Bar, $300 \mathrm{~nm}$. (insets) Magnifications of distinct areas (marked by white boxes) of the membrane show striated areas (red parallel lines) that resemble the pattern of recombinant Pill and Lspl structures. Bars, $100 \mathrm{~nm}$. (B) Immunolabeling of plasma membranes of cells expressing Pill-GFP using anti-GFP antibodies. Yellow circles highlight 18 -nm gold particles for better visibility. Bars, $100 \mathrm{~nm}$. ( $A$ and $B$ ) The structures are visible on the flat membrane as well as on the side of large invaginations (arrowheads). (C) DEEM images showing views on the plasma membrane from different perspectives. (top) View from the outside of a cell onto the inner leaflet of the plasma membrane. (bottom) View from the cytoplasm (marked as c) onto the plasma membrane (marked as $\mathrm{m}$; red/cyan 3D glasses are recommended for 3D view, as well as for $D$ ). Bars, $300 \mathrm{~nm}$. (D) View from the cytoplasm onto an eisosome at the plasma membrane. Arrowheads indicate how the plasma membrane protrudes underneath the eisosome protein coat to form a groove instead of a closed tube. Bar, $100 \mathrm{~nm}$.
A

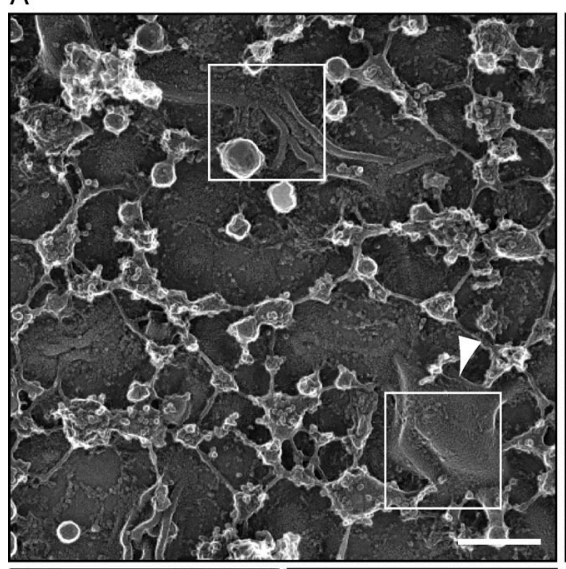

B
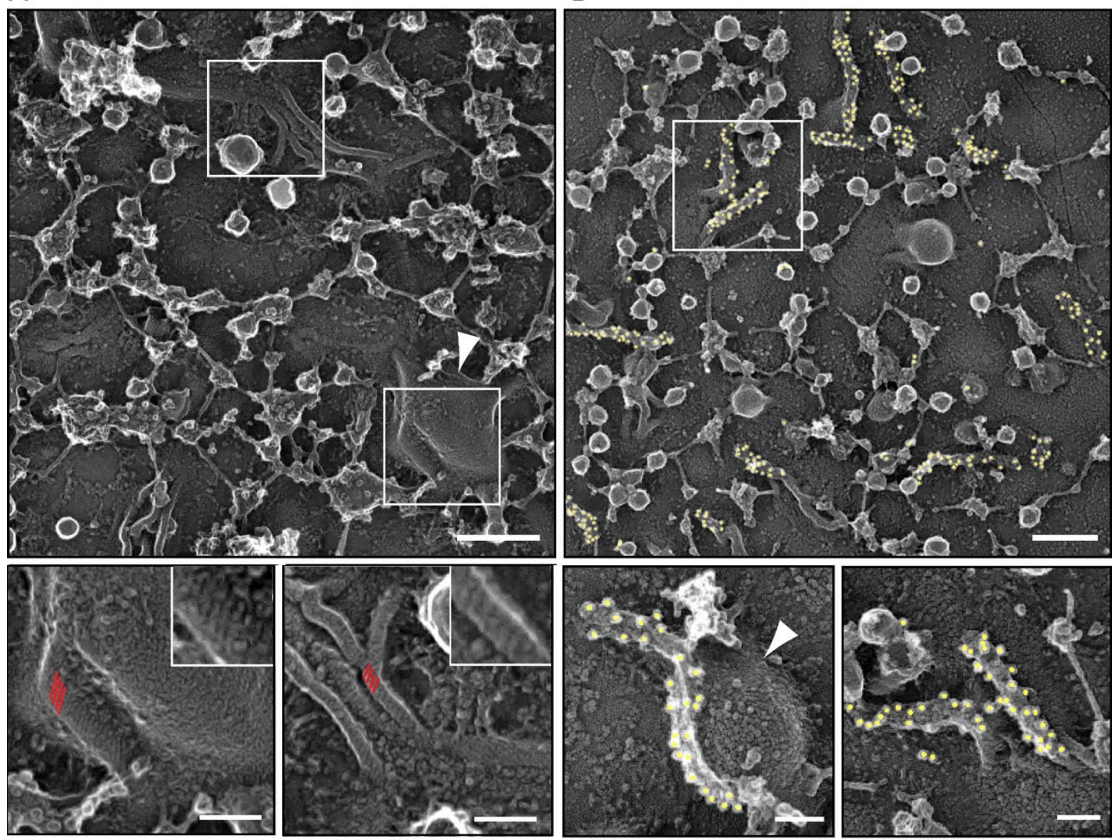

C

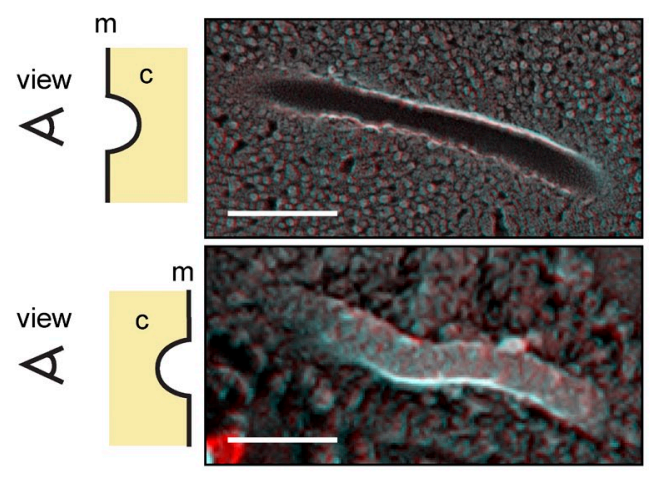

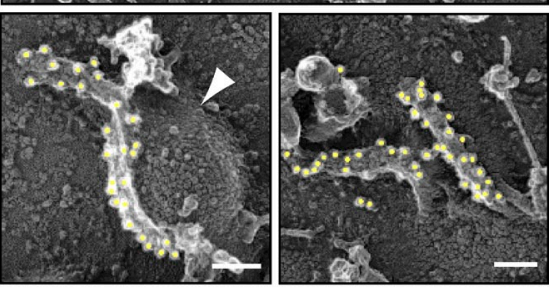

D

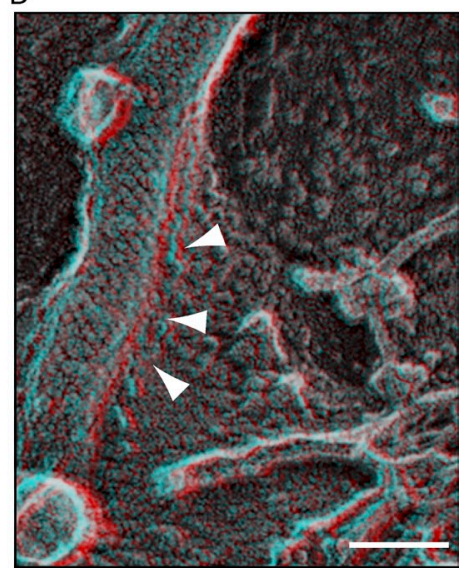

Pil1, which leads to formation of thin helices. Similarly, pil1 $\Delta \mathrm{N}$ formed only thin filaments, further suggesting that the N-terminal segment containing two of phosphorylation sites is required for interaction 3. These data may explain eisosome disassembly after overexpression of Pkh kinases, addition of myriocin, or other treatments that increase Pil1 phosphorylation (Walther et al., 2007; Luo et al., 2008; Fröhlich et al., 2009).

Pil1 and Lsp1 are most likely targeted to the plasma membrane by efficient membrane binding (Fig. 8 B). We predict that, initially, dimers or thin filament pieces interact with membranes and assemble in vitro into a stable helix with a membrane tubule inside or in vivo into a furrowlike lattice (Fig. 8 B). Several lines of evidence suggest that Pil1 and Lsp1 interact with $\mathrm{PI}(4,5) \mathrm{P}_{2}$ : (a) Pil1 and Lsp1 tubulate liposomes containing low amounts of $\mathrm{PI}(4,5) \mathrm{P}_{2}$; (b) fluorescence spectroscopy of NBD-labeled Pil1 yields a strong signal consistent with membrane binding when $\mathrm{PI}(4,5) \mathrm{P}_{2}$-containing liposomes are present; (c) in sedimentation assays, Lsp1 interacts more strongly with $\mathrm{PI}(4,5) \mathrm{P}_{2}$-containing liposomes than with those containing other types of charged lipids at the same concentration; (d) inactivation of Mss4, leading to $\mathrm{PI}(4,5) \mathrm{P}_{2}$ depletion, has a strong effect on eisosome localization in vivo; (e) conversely, deletion of two $\mathrm{PI}(4,5) \mathrm{P}_{2}$ phosphatases (SJL1 and SJL2) and increased $\mathrm{PI}(4,5) \mathrm{P}_{2}$ levels lead to enlarged Pil1-GFP assemblies; and (f) PILI and SJL1 show highly similar genetic interaction profiles in independently generated E-MAP datasets.

How can self-assembly of Pil1 and Lsp1 promote formation or stabilization of curved membranes, such as tubules and furrows? Two mechanisms for BAR domain-induced membrane bending are currently considered (Kozlov et al., 2010): protein scaffolding of the membrane and insertion of a wedge into one leaflet of the lipid bilayer. Both of these mechanisms are used by other BAR domain-containing proteins (Peter et al., 2004). Our reconstructions of Lsp1 with and without bound membranes show very similar structures, suggesting a scaffold mechanism. In addition, a part of the protein at the concave surface of the Pil1 or Lsp1 coat may be inserted as a wedge in one leaflet of the bilayer, for example, represented by the part of Lsp1 observed close to the membrane surface. Consistent with this notion, we found that a membrane-facing $\mathrm{N}$-terminal segment 
of Pil1 or Lsp1 is required for efficient membrane binding of the proteins in addition to a positive patch of amino acids on the concave surface of the BAR domain. As a consequence of the insertion of a membrane wedge, the order of the outer membrane leaflet could be disordered, leading to absence of resolved density in this region and thus potentially explaining the gap apparent in our reconstructions between the lipid layer to the protein scaffold.

Many of these considerations are based on similarity between the models of Pil1 and Lsp1 assemblies in vitro and the structure of eisosomes, forming membrane furrows in yeast (Strádalová et al., 2009). This interpretation is supported by (a) a very similar structure for recombinant Pil1 and Lsp1 assemblies as for purified eisosomes isolated from yeast cells, (b) alterations of the in vitro structure caused by phosphomimicking mutations in Pil1, consistent with the phenotype of these mutations in yeast, and (c) the striated pattern of eisosomes on plasma membrane furrows or the cytoplasmic side of the plasma membrane, which resembles the pattern of thick helices formed by the recombinant proteins. Despite the overall close resemblance of the structures, there are at least two important differences. First, eisosomes contain both Pil1 and Lsp1 proteins. Thus, in vivo, the building blocks of the lattice could be Pil1 and Lsp1 heterodimers or a mixture of both types of homodimers, rather than a single species of homodimers present in vitro, and the different properties of the two proteins could be used to modulate the assembly. Second, whereas the in vitro filaments are closed cylinders coating a membrane tubule, eisosomes in vivo coat a membrane furrow, which likely resembles a half-cylinder. Attachment of the membrane to the cell wall and the large turgor pressure could prevent the closure of the lattice to a helix similar to the ones seen in vitro. Alternatively, a transition phase of specific lipid or protein composition at the eisosome boundary could prevent the closure of the tubules. It remains possible that the furrows are closed to a tube or otherwise remodeled as a result of the rearrangement of the proteins under some conditions. Such remodeling may be supported by flexibility of Lsp1 BAR domain tips and arrangement of subunits, reflected in tube diameter variability, observed in vitro. Interestingly, during uptake of the membrane dye FM4-64, some but not all eisosomes are labeled by bright dye-containing foci, indicating that the plasma membrane has a different structure at those sites.

From our work, several intriguing similarities between eisosomes and endophilin/amphyphysin BAR domain proteins emerge. Both protein families consist of BAR domains, can assemble into a scaffold on membranes, are connected to $\mathrm{PI}(4,5) \mathrm{P}_{2^{-}}$ rich membranes, and function with synaptojanin proteins (Itoh et al., 2005). Additionally, both sets of proteins were linked to endocytosis, but their deletions have mild defects on protein uptake in most systems (Schuske et al., 2003; Verstreken et al., 2003; Walther et al., 2006; Grossmann et al., 2008; Brach et al., 2011). Endophilin recruits synaptojanin to endocytic sites through an SH3 domain (Schuske et al., 2003). Neither Pil1 nor Lsp1 contains such a domain. However, it was recently reported that the membrane-bending activity of endophilin particularly is important for many functions of the protein in Caenorhabditis elegans (Bai et al., 2010), and we now find that eisosome

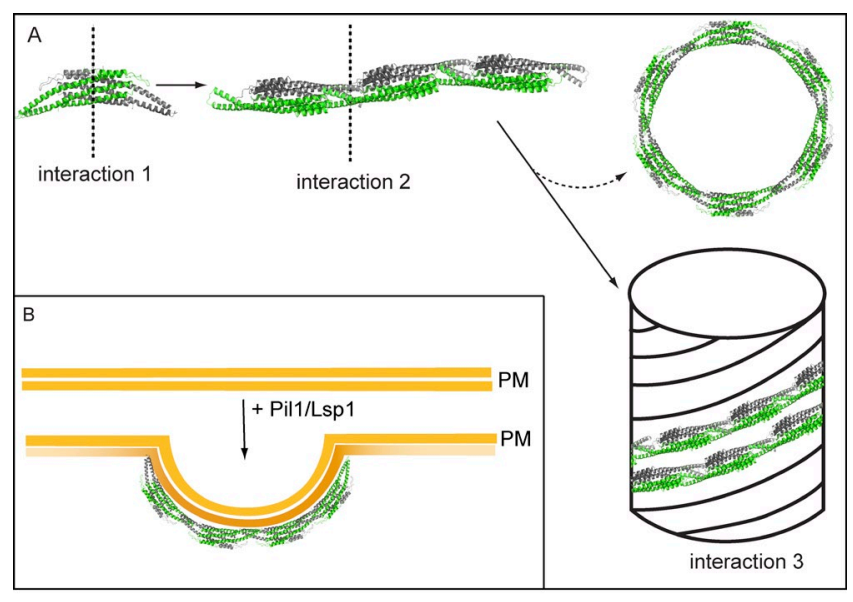

Figure 8. Model for the assembly of eisosomes on the plasma membrane. (A) The assembly of eisosomes can be separated conceptually into three steps: interactions of the proteins to form dimers (interaction 1), association of dimers to form thin filaments (interaction 2), and assembly into helices (interaction 3). Rings observed for Pill are interpreted in this model as side products of the filament-to-helix assembly. (B) On the plasma membrane (PM), main eisosome components assemble into a scaffold similar to a half helix (see Discussion for details).

proteins also bend membranes. Based on these considerations and the similarity of interaction profiles between pills and $s j l 1 \Delta$, it is possible that both genes participate in the same process, e.g., in $\mathrm{PI}(4,5) \mathrm{P}_{2}$ turnover. Interestingly, membrane curvature, for example, caused by interaction with endophilin, aids synaptojanin activity (Chang-Ileto et al., 2011).

In summary, formation of an eisosome protein scaffold can mechanistically explain how the yeast plasma membrane is organized in domains of distinct composition, in particular for the MCC. We posit that membrane binding and assembly by Pill and Lsp1 will create a specific environment in the overlaying MCC, which is locally curved and may have increased $\mathrm{PI}(4,5) \mathrm{P}_{2}$ concentration as a result of the presence of many binding sites for this lipid. This special environment then drives formation of the MCC domain.

\section{Materials and methods}

\section{Yeast strains}

All yeast strains were derived from the W303 or $\mathrm{S} 288 \mathrm{C}$ strain background using PCR-based modification (Janke et al., 2004) and are listed in Table S1. Cells were grown for normal strains at $30^{\circ} \mathrm{C}$ or at $24^{\circ} \mathrm{C}$ for temperature-sensitive mutants, with shaking in standard rich medium (yeast peptone dextrose [YPD]) or in synthetic medium (Synthetic complete) supplemented with adenine, uracil, histidine, leucine, and tryptophane.

\section{Protein expression and purification}

Recombinant Pill and Lsp 1 were expressed in Escherichia coli BL2 1 (DE3) cells using pGEX-6P-1 vector (GE Healthcare) and IPTG induction for $3.5 \mathrm{~h}$ at $25^{\circ} \mathrm{C}$. Pellets were frozen in liquid nitrogen and stored at $-80^{\circ} \mathrm{C}$. Cell pellets were thawed and broken up in lysis buffer $1500 \mathrm{mM} \mathrm{KoAc}$, $2 \mathrm{mM} \mathrm{MgAc}, 100 \mathrm{mM}$ Hepes, pH 7.4, and $0.2 \mathrm{mM}$ PMSF) by several passages through a microfluidizer (Microfluidics). Proteins were affinity purified with glutathione Sepharose (GE Healthcare), and the GST tag was cleaved off using PreScission protease. Eluted proteins were further purified by anion exchange chromatography on a HiTrap Q FF column (GE Healthcare) using an AKTA purifier system (GE Healthcare). Fractions were dialyzed overnight (150 mM KoAc, $2 \mathrm{mM} \mathrm{MgAc}, 20 \mathrm{mM}$ Hepes, $\mathrm{pH} 7.4$, and $5 \%$ glycerol) and concentrated to $30 \mu \mathrm{M}$ in centrifugal filter devices (Amicon Ultra; Millipore). Aliquots were frozen in liquid nitrogen and stored at $-80^{\circ} \mathrm{C}$. 


\section{Sedimentation velocity gradients}

$100 \mu \mathrm{g}$ of recombinant protein was loaded in $150 \mu$ of buffer $(150 \mathrm{mM}$ KoAc, $2 \mathrm{mM} \mathrm{MgAc}$, and $20 \mathrm{mM}$ Hepes, pH7.4) on $12 \mathrm{ml}$ of $10-40 \%$ sucrose gradient. Gradients were generated using a gradient master (BioComp Systems, Inc.). The gradients were spun in an ultracentrifuge (WX Ultra Series; Thermo Fisher Scientific) equipped with a TH-641 rotor at $40,000 \mathrm{rpm}$ at $4^{\circ} \mathrm{C}$ for $3.5 \mathrm{~h}$. 1-ml fractions were manually pipetted, and proteins were precipitated using TCA and analyzed by SDS-PAGE and Coomassie blue staining.

\section{Vesicle preparation}

Lipids in desired ratios (mole/mole) were mixed and dried in glass vials under a nitrogen stream. Before use, mixtures were desiccated under a vacuum for $2 \mathrm{~h}$ and hydrated in buffer. To obtain unilamellar vesicles, lipids were subjected to 5 cycles of freeze-thaw and extruded through a 200-nm pore-size polycarbonate filter (GE Healthcare) using a mini-extruder (Avanti Polar Lipids, Inc.).

\section{Fluorescence microscopy}

For fluorescence microscopy, cells were grown in synthetic media to $\mathrm{OD}_{600}=0.5$, mounted on coverslips covered with concanavalin $\mathrm{A}$, and imaged either with a laser-based spinning-disk microscope (Andor Technology, TILL Photonics, and Agilent Technologies) using a 100x total internal reflection fluorescence microscopy objective (1.45 NA; Olympus) or using a DeltaVision system (Applied Precision) equipped with a microscope (IX-71; Olympus), a 1.35 NA 100x objective (Olympus), and a CoolSNAP HQ camera (Photometrics).

\section{Spin-down assay}

Liposome samples $(4 \mathrm{mM})$ were incubated in the presence or absence of $3.75 \mu \mathrm{M}$ recombinant protein in $40 \mu$ of buffer $(150 \mathrm{mM} \mathrm{KoAc}, 2 \mathrm{mM}$ $\mathrm{MgAc}$, and $20 \mathrm{mM}$ Hepes, pH 7.4) for $20 \mathrm{~min}$ at room temperature. Samples were centrifuged in an ultracentrifuge (Discovery M120 SE; Thermo Fisher Scientific) using an S120-AT3 rotor at $85,000 \mathrm{rpm}$ at $4^{\circ} \mathrm{C}$ for $30 \mathrm{~min}$. Pellets and supernatants were separated and brought to $42 \mu$ with SDS loading buffer, and both fractions were analyzed by SDS-PAGE and Coomassie blue staining.

\section{EM}

For negative staining, $3.5 \mu$ of purified Pill or Lspl protein or protein liposome suspension was added on glow-discharged continuous carboncoated Cu grids. After blotting with filter paper, the grid surface was stained with $1 \%$ uranyl acetate solution. Images were taken at magnifications of 23,000-31,000x using either a microscope (C120; Philips) equipped with a $1 \mathrm{kx} 1 \mathrm{k}$ charge-coupled device camera (Gatan, Inc) and operated at 120 kiloelectron volts or on a microscope (Tecnai F20; FEI Company) equipped with a $4 \mathrm{k} \times 4 \mathrm{k}$ charge-coupled device camera (FEI Company) and operated at 200 kiloelectron volts. For cryo-EM, a 3-pl aliquot of purified Lspl or Pill protein or protein-liposome suspension was pipetted on a glow-discharged holey carbon-coated EM grid (C-Flat; Protochips, Incorporated). For cryoelectron tomography, $1 \mu \mathrm{l}$ of diluted BSA-coated colloidal gold particles (10 nm in diameter) was added. Excess suspension was blotted with a filter paper, and the sample was vitrified by plunging it rapidly into liquid ethane. Cryo-EM was performed at liquid nitrogen temperature using a microscope (Tecnai F2O) equipped with a $4 \mathrm{kx} 4 \mathrm{k}$ charge-coupled device camera (FEI Company) operated at 200 kiloelectron volts. Single low-dose images $\left(20 \mathrm{e}^{-} / \mathrm{A}^{2}\right)$ or tilt series of 61 images from -60 to +60 degrees $\left(80-130 \mathrm{e}^{-} / \mathrm{A}^{2}\right)$ were collected with SerialEM (Mastronarde, 2005) at 1-3 $\mu \mathrm{m}$ of underfocus and at a nominal magnification of 50,000 , giving a calibrated pixel size of $0.22 \mathrm{~nm}$.

\section{Tomography and subtomogram averaging}

Tomographic reconstructions were calculated from the tilt series in IMOD (Mastronarde, 2005) and down-sampled by a factor of three, giving a final pixel size of $0.66 \mathrm{~nm}$. Three filaments, oriented in the direction of the tilt axis, were traced in a tomographic reconstruction of Lsp 1. To calculate an averaged structure of Lspl filament, 128 overlapping 3D filament segments $(100 \times 100 \times 100$ voxels, with a $90 \%$ overlap) were extracted. A cylindrical average of all segments was used as a reference in crosscorrelation alignment, calculated using a custom Bsoft (Heymann and Belnap, 2007) program, Jsubtomo (available upon request; Huiskonen et al., 2010). The average of all aligned segments acted as a reference for a subsequent 20 rounds of alignment and averaging. The angle defining the orientation of the filament segment around the filament long axis was initially randomized, and changes of only 16 degrees were allowed during the alignment. This angular constraint and the orientation of the filament along the tilt axis ensured that the missing wedge artifact present in all tomographic data was nearly evenly averaged in the final structure, yielding an isotropic resolution in all directions.

\section{Helical reconstruction}

218 cryo-EM images were computationally down-sampled by a factor of two, giving a final pixel size of $0.44 \mathrm{~nm}$. Contrast transfer function parameters were determined in the micrographs, and corresponding image distortions were corrected for in Bsoft. Filaments were traced in the images and cut into overlapping segments (90\% overlap). Subimages (150 $\times 150$ pixels), each containing a filament segment, were extracted (Table S2). Subimages were rotated so that the long axis of the segment was parallel to the image vertical axis. Rotated subimages were aligned horizontally to center the segments. The average of all segments was used as a template, and the process of averaging and alignment was iterated five times in SPIDER (Frank et al., 1996). Horizontally aligned images were subjected to multivariate data analysis and classification in SPIDER. The segments were first classified into 100 classes for each sample. The size of the Lspl dataset was reduced from 55,000 to 20,000 segments for classification. Class averages revealed variation in the datasets, most notably variation in the filament diameter. The initial horizontal alignment was improved by multireference alignment, using the first class means as references. These more accurately aligned segments were reclassified into 100 classes using the first 20 eigenimages. 3D reconstructions were generated by Fourier-Bessel analysis of the refined class averages using the Burnham-Brandeis Helical Package (designed by N. Volkmann, Sanford-Burnham Medical Research Institute, La Jolla, CA), an updated version of the original Brandeis Helical Package (Owen et al., 1996). In an attempt to detect all most prominent helical symmetries in the data, all Fourier transforms of class averages displaying strong layer lines consistent with either an even or an odd Bessel order were analyzed. Layer line indexes were assigned for 6 Fourier transforms of $\mathrm{Lspl}$ and $\mathrm{Lsp}-\mathrm{PI}(4,5) \mathrm{P}_{2}$ class averages and for 12 Fourier transforms of Pill-PI $(4,5) \mathrm{P}_{2}$. In some cases, a few different assignments of Bessel orders were possible, and they were all considered in further analysis. 3D density maps were calculated from the Fourier transforms. The features of the repeating structural unit were similar between the density maps and also to the tomographic reconstruction of an Lspl filament, confirming that the layer line indexes and Bessel orders were assigned correctly. In the few ambiguous cases, the assignment, which resulted in a density map with consistent features to the other maps, was chosen to be the correct assignment.

Fourier-Bessel analysis provided multiple low-resolution template structures for the 3D reconstruction using IHRSR (Egelman, 2007). Several template structures with unique helical parameters were used: two for Lsp 1, three for Lsp 1-PI(4,5) $\mathrm{P}_{2}$, and four for Pil 1-PI(4,5) $\mathrm{P}_{2}$ (Table S3). The filament segments that had been prealigned horizontally were classified into groups with different helical parameters and symmetries using projections from the template structures as references. Segment positions were adjusted horizontally to account for inaccuracies in the initial alignment against a common reference. IHRSR was run for five iterations for each group of segments. $80 \%$ of the best-correlating segments were chosen at each round to the reconstruction to count in possible inaccuracies in classification. The filament segments were reclassified against the refined models and five iterations of IHRSR were run. Because the starting models already had the correct helical symmetries present in the data, the helical symmetry parameters were kept constant during the iterations.

\section{X-ray structure fitting to EM maps}

Twofold symmetric Lspl dimers (AA, BB, and CC) were first generated from the three chains in the crystallographic asymmetric unit $(A, B$, and $C)$ by superposition of the chains on the crystallographic dimer $(A B)$ in the program LSQMAN (G.J. Kleywegt, Uppsala Software Factory, Sweden; Kleywegt, 1996). Only the core part of the dimer was used in the superposition. All three types of dimers-AA, BB and, CC-were fitted computationally on the six different cryo-EM density maps of Lsp 1 and Pill filaments. Fitting was performed in CoLoRes (Situs package; Chacón and Wriggers, 2002) by calculating Laplacian-filtered correlation between the $x$-ray structure and the cryo-EM reconstruction to the resolution limit of each reconstruction. Helical symmetry of each of the reconstructions was applied on the best-fitting x-ray structure to generate atomic models of the filaments. For visualization in the University of California San Francisco Chimera program, the isosurface levels of cryo-EM reconstructions were set to correspond to the total mass of fitted Lspl structures, taking into account the presence of a membrane in some of the structures. 


\section{E-MAP}

E-MAP datasets were either from Aguilar et al. (2010) or generated as previously described in Collins et al. (2006).

\section{Spheroplasting and sample preparation for DEEM}

For generation of yeast spheroplasts, cells were grown by shaking at $30^{\circ} \mathrm{C}$ to $\mathrm{OD}_{600}=0.5$ in YPD and treated with zymolyase for $10 \mathrm{~min}$ (modified from Ogg et al. [1992]). "Unroofing" of spheroplasts, as well as antibody decoration, freezing, replicating, and imaging of the samples, was performed as previously described (Ogg et al., 1992; Hanson et al., 2008). The primary antibodies used were rabbit anti-GFP (Invitrogen) or rabbit anti-Pill (Walther et al., 2007). For freeze-fracture DEEM, cells were grown to $\mathrm{OD}_{600}=0.5$ in YPD and quick-frozen by abrupt application of the sample on a coverslip onto a block of ultrapure copper cooled to liquid helium temperature (Heuser, 1989) before deep etching and platinum replication.

\section{Data deposition}

The cryo-EM reconstructions (Table S3) have been deposited in the Electron Microscopy Data Bank at the European Bioinformatics Institute under accession codes EMD-1865 (Lsp1 \#1), EMD-1866 (Lspl \#2), EMD-1867 (Lsp 1-liposome \#1), and EMD-1868 (Pil1-liposome \#1).

\section{Online supplemental material}

Fig. S1 shows that Pill and Lsp1 form stable filaments of variable diameter in vitro and that their localization depends on plasma membrane $\mathrm{PI}(4,5) \mathrm{P}_{2}$ Fig. S2 shows that Mrp8 does not localize in eisosomes and is not required for normal eisosomes at the plasma membrane. Fig. S3 shows that the yeast plasma membrane exhibits abundant furrows that depend on PIL 1 and that immunogold-labeled Pil 1 and Lspl-GFP localize in elongated structures on plasma membranes from unroofed yeast cells. Videos 1, 2, and 3 show time lapse imaging of eisosomes marked by Pil 1-GFP at different time points during Mss 4 inactivation. Table S1 contains all yeast strains used in this study. Tables S2 and S3 present the data, collection, and reconstruction statistics for helical reconstruction. Online supplemental material is available at http://www.jcb.org/cgi/content/full/jcb.201104040/DC1.

We thank Pietro DeCamilli, Robert Farese Jr., Shawn Ferguson, Boumediene Soufi, and Sebastian Schuck for critical reading of the manuscript and suggestions. We would like to especially thank Michael Shales for help in preparing the E-MAP figure and Judith Short and Edward Egelman for valuable suggestions concerning reconstruction of filament structures.

This work was supported by the Boehringer Ingelheim Fonds (L. Karotki), the Academy of Finland (grant 218080 to J.T. Huiskonen), the Max Planck Society (T.C. Walther), the German Research Foundation (T.C. Walther and K. Grünewald), and the Minna-James-Heineman Foundation (T.C. Walther).

Submitted: 8 April 2011

Accepted: 31 October 2011

\section{References}

Aguilar, P.S., F. Fröhlich, M. Rehman, M. Shales, I. Ulitsky, A. Olivera-Couto, H. Braberg, R. Shamir, P. Walter, M. Mann, et al. 2010. A plasmamembrane E-MAP reveals links of the eisosome with sphingolipid metabolism and endosomal trafficking. Nat. Struct. Mol. Biol. 17:901-908. http://dx.doi.org/10.1038/nsmb.1829

Bai, J., Z. Hu, J.S. Dittman, E.C. Pym, and J.M. Kaplan. 2010. Endophilin functions as a membrane-bending molecule and is delivered to endocytic zones by exocytosis. Cell. 143:430-441. http://dx.doi.org/10.1016/ j.cell.2010.09.024

Berchtold, D., and T.C. Walther. 2009. TORC2 plasma membrane localization is essential for cell viability and restricted to a distinct domain. Mol. Biol. Cell. 20:1565-1575. http://dx.doi.org/10.1091/mbc .E08-10-1001

Brach, T., T. Specht, and M. Kaksonen. 2011. Reassessment of the role of plasma membrane domains in the regulation of vesicular traffic in yeast. $J$. Cell Sci. 124:328-337. http://dx.doi.org/10.1242/jcs.078519

Chacón, P., and W. Wriggers. 2002. Multi-resolution contour-based fitting of macromolecular structures. J. Mol. Biol. 317:375-384. http://dx.doi .org/10.1006/jmbi.2002.5438

Chang-Ileto, B., S.G. Frere, R.B. Chan, S.V. Voronov, A. Roux, and G. Di Paolo. 2011. Synaptojanin 1-mediated PI(4,5)P2 hydrolysis is modulated by membrane curvature and facilitates membrane fission. Dev. Cell. 20:206-218. http://dx.doi.org/10.1016/j.devcel.2010.12.008
Collins, S.R., M. Schuldiner, N.J. Krogan, and J.S. Weissman. 2006. A strategy for extracting and analyzing large-scale quantitative epistatic interaction data. Genome Biol. 7:R63. http://dx.doi.org/10.1186/gb-2006-7-7-r63

de Godoy, L.M., J.V. Olsen, J. Cox, M.L. Nielsen, N.C. Hubner, F. Fröhlich, T.C. Walther, and M. Mann. 2008. Comprehensive mass-spectrometrybased proteome quantification of haploid versus diploid yeast. Nature. 455:1251-1254. http://dx.doi.org/10.1038/nature07341

DeRosier, D.J., and P.B. Moore. 1970. Reconstruction of three-dimensional images from electron micrographs of structures with helical symmetry. J. Mol. Biol. 52:355-369. http://dx.doi.org/10.1016/0022-2836 (70)90036-7

Egelman, E.H. 2007. The iterative helical real space reconstruction method: Surmounting the problems posed by real polymers. J. Struct. Biol. 157:83-94. http://dx.doi.org/10.1016/j.jsb.2006.05.015

Frank, J., M. Radermacher, P. Penczek, J. Zhu, Y. Li, M. Ladjadj, and A. Leith. 1996. SPIDER and WEB: Processing and visualization of images in 3D electron microscopy and related fields. J. Struct. Biol. 116:190-199. http://dx.doi.org/10.1006/jsbi.1996.0030

Fröhlich, F., K. Moreira, P.S. Aguilar, N.C. Hubner, M. Mann, P. Walter, and T.C. Walther. 2009. A genome-wide screen for genes affecting eisosomes reveals Nce102 function in sphingolipid signaling. J. Cell Biol. 185:1227-1242. http://dx.doi.org/10.1083/jcb.200811081

Frost, A., R. Perera, A. Roux, K. Spasov, O. Destaing, E.H. Egelman, P. De Camilli, and V.M. Unger. 2008. Structural basis of membrane invagination by F-BAR domains. Cell. 132:807-817. http://dx.doi.org/10.1016/ j.cell.2007.12.041

Gallop, J.L., and H.T. McMahon. 2005. BAR domains and membrane curvature: Bringing your curves to the BAR. Biochem. Soc. Symp. 72:223-231.

Ghaemmaghami, S., W.K. Huh, K. Bower, R.W. Howson, A. Belle, N. Dephoure, E.K. O'Shea, and J.S. Weissman. 2003. Global analysis of protein expression in yeast. Nature. 425:737-741. http://dx.doi.org/10.1038/ nature 02046

Grossmann, G., M. Opekarová, J. Malinsky, I. Weig-Meckl, and W. Tanner. 2007. Membrane potential governs lateral segregation of plasma membrane proteins and lipids in yeast. EMBO J. 26:1-8. http://dx.doi.org/10.1038/ sj.emboj.7601466

Grossmann, G., J. Malinsky, W. Stahlschmidt, M. Loibl, I. Weig-Meckl, W.B. Frommer, M. Opekarová, and W. Tanner. 2008. Plasma membrane microdomains regulate turnover of transport proteins in yeast. J. Cell Biol. 183:1075-1088. http://dx.doi.org/10.1083/jcb.200806035

Hanson, P.I., R. Roth, Y. Lin, and J.E. Heuser. 2008. Plasma membrane deformation by circular arrays of ESCRT-III protein filaments. J. Cell Biol. 180:389-402. http://dx.doi.org/10.1083/jcb.200707031

Heuser, J.E. 1989. Development of the quick-freeze, deep-etch, rotary-replication technique of sample preparation for 3-D electron microscopy. Prog. Clin. Biol. Res. 295:71-83.

Heymann, J.B., and D.M. Belnap. 2007. Bsoft: Image processing and molecular modeling for electron microscopy. J. Struct. Biol. 157:3-18. http://dx.doi .org/10.1016/j.jsb.2006.06.006

Hua, W., D. Sheff, D. Toomre, and I. Mellman. 2006. Vectorial insertion of apical and basolateral membrane proteins in polarized epithelial cells revealed by quantitative 3D live cell imaging. J. Cell Biol. 172:1035-1044. http://dx.doi.org/10.1083/jcb.200512012

Huiskonen, J.T., J. Hepojoki, P. Laurinmäki, A. Vaheri, H. Lankinen, S.J Butcher, and K. Grünewald. 2010. Electron cryotomography of Tula hantavirus suggests a unique assembly paradigm for enveloped viruses. J. Virol. 84:4889-4897. http://dx.doi.org/10.1128/JVI.00057-10

Itoh, T., K.S. Erdmann, A. Roux, B. Habermann, H. Werner, and P. De Camilli. 2005. Dynamin and the actin cytoskeleton cooperatively regulate plasma membrane invagination by BAR and F-BAR proteins. Dev. Cell. 9:791804. http://dx.doi.org/10.1016/j.devcel.2005.11.005

Janke, C., M.M. Magiera, N. Rathfelder, C. Taxis, S. Reber, H. Maekawa, A. Moreno-Borchart, G. Doenges, E. Schwob, E. Schiebel, and M. Knop. 2004. A versatile toolbox for PCR-based tagging of yeast genes: New fluorescent proteins, more markers and promoter substitution cassettes. Yeast. 21:947-962. http://dx.doi.org/10.1002/yea.1142

Kleywegt, G.J. 1996. Use of non-crystallographic symmetry in protein structure refinement. Acta Crystallogr. D Biol. Crystallogr. 52:842-857. http:// dx.doi.org/10.1107/S0907444995016477

Kozlov, M.M., H.T. McMahon, and L.V. Chernomordik. 2010. Protein-driven membrane stresses in fusion and fission. Trends Biochem. Sci. 35:699706. http://dx.doi.org/10.1016/j.tibs.2010.06.003

Luo, G., A. Gruhler, Y. Liu, O.N. Jensen, and R.C. Dickson. 2008. The sphingolipid long-chain base-Pkh1/2-Ypk1/2 signaling pathway regulates eisosome assembly and turnover. J. Biol. Chem. 283:10433-10444. http://dx.doi.org/10.1074/jbc.M709972200

Malínská, K., J. Malínský, M. Opekarová, and W. Tanner. 2003. Visualization of protein compartmentation within the plasma membrane of living yeast 
cells. Mol. Biol. Cell. 14:4427-4436. http://dx.doi.org/10.1091/mbc .E03-04-0221

Mastronarde, D.N. 2005. Automated electron microscope tomography using robust prediction of specimen movements. J. Struct. Biol. 152:36-51. http://dx.doi.org/10.1016/j.jsb.2005.07.007

Norbeck, J., and A. Blomberg. 1997. Metabolic and regulatory changes associated with growth of Saccharomyces cerevisiae in $1.4 \mathrm{M} \mathrm{NaCl}$. Evidence for osmotic induction of glycerol dissimilation via the dihydroxyacetone pathway. J. Biol. Chem. 272:5544-5554. http://dx.doi .org/10.1074/jbc.272.9.5544

Ogg, S.C., M.A. Poritz, and P. Walter. 1992. Signal recognition particle receptor is important for cell growth and protein secretion in Saccharomyces cerevisiae. Mol. Biol. Cell. 3:895-911.

Olivera-Couto, A., M. Graña, L. Harispe, and P.S. Aguilar. 2011. The eisosome core is composed of BAR domain proteins. Mol. Biol. Cell. 22:23602372. http://dx.doi.org/10.1091/mbc.E10-12-1021

Owen, C.H., D.G. Morgan, and D.J. DeRosier. 1996. Image analysis of helical objects: The Brandeis Helical Package. J. Struct. Biol. 116:167-175. http://dx.doi.org/10.1006/jsbi.1996.0027

Peter, B.J., H.M. Kent, I.G. Mills, Y. Vallis, P.J. Butler, P.R. Evans, and H.T. McMahon. 2004. BAR domains as sensors of membrane curvature: The amphiphysin BAR structure. Science. 303:495-499. http://dx.doi.org/ 10.1126/science. 1092586

Ren, G., P. Vajjhala, J.S. Lee, B. Winsor, and A.L. Munn. 2006. The BAR domain proteins: Molding membranes in fission, fusion, and phagy. Microbiol. Mol. Biol. Rev. 70:37-120. http://dx.doi.org/10.1128/ MMBR.70.1.37-120.2006

Schuske, K.R., J.E. Richmond, D.S. Matthies, W.S. Davis, S. Runz, D.A. Rube, A.M. van der Bliek, and E.M. Jorgensen. 2003. Endophilin is required for synaptic vesicle endocytosis by localizing synaptojanin. Neuron. 40:749762. http://dx.doi.org/10.1016/S0896-6273(03)00667-6

Stefan, C.J., A. Audhya, and S.D. Emr. 2002. The yeast synaptojanin-like proteins control the cellular distribution of phosphatidylinositol $(4,5)$-bisphosphate. Mol. Biol. Cell. 13:542-557. http://dx.doi.org/10.1091/mbc.01-10-0476

Strádalová, V., W. Stahlschmidt, G. Grossmann, M. Blazíková, R. Rachel, W. Tanner, and J. Malinsky. 2009. Furrow-like invaginations of the yeast plasma membrane correspond to membrane compartment of Can1. J. Cell Sci. 122:2887-2894. http://dx.doi.org/10.1242/jcs.051227

Verstreken, P., T.W. Koh, K.L. Schulze, R.G. Zhai, P.R. Hiesinger, Y. Zhou, S.Q. Mehta, Y. Cao, J. Roos, and H.J. Bellen. 2003. Synaptojanin is recruited by endophilin to promote synaptic vesicle uncoating. Neuron. 40:733-748. http://dx.doi.org/10.1016/S0896-6273(03)00644-5

Walther, T.C., J.H. Brickner, P.S. Aguilar, S. Bernales, C. Pantoja, and P. Walter. 2006. Eisosomes mark static sites of endocytosis. Nature. 439:998-1003. http://dx.doi.org/10.1038/nature04472

Walther, T.C., P.S. Aguilar, F. Fröhlich, F. Chu, K. Moreira, A.L. Burlingame, and P. Walter. 2007. Pkh-kinases control eisosome assembly and organization. EMBO J. 26:4946-4955. http://dx.doi.org/10.1038/sj.emboj.7601933

Wang, H., B. Kakaradov, S.R. Collins, L. Karotki, D. Fiedler, M. Shales, K.M. Shokat, T.C. Walther, N.J. Krogan, and D. Koller. 2009. A complex-based reconstruction of the Saccharomyces cerevisiae interactome. Mol. Cell. Proteomics. 8:1361-1381. http://dx.doi.org/10.1074/mcp.M800490MCP200

Zhang, X., R.L. Lester, and R.C. Dickson. 2004. Pil1p and Lsp1p negatively regulate the 3-phosphoinositide-dependent protein kinase-like kinase Pkh1p and downstream signaling pathways Pkclp and Ypk1p. J. Biol. Chem. 279:22030-22038. http://dx.doi.org/10.1074/jbc.M400299200

Ziółkowska, N.E., L. Karotki, M. Rehman, J.T. Huiskonen, and T.C. Walther. 2011. Eisosome-driven plasma membrane organization is mediated by BAR domains. Nat. Struct. Mol. Biol. 18:854-856. http://dx.doi.org/10.1038/ nsmb. 2080 

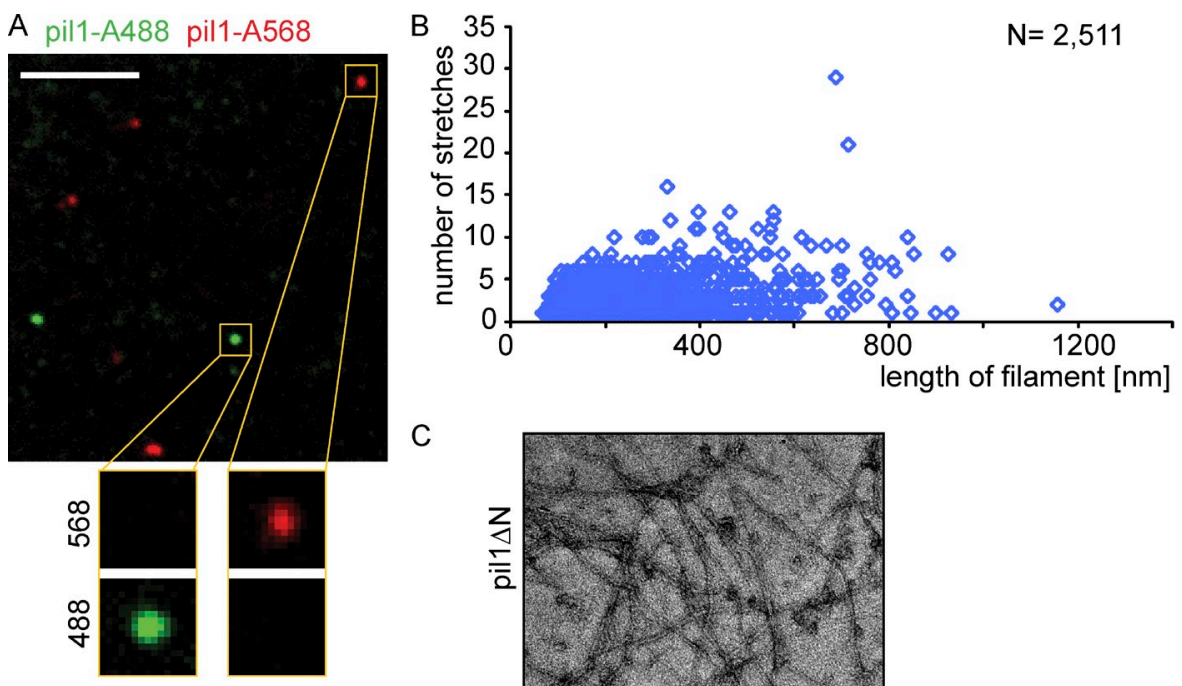

C
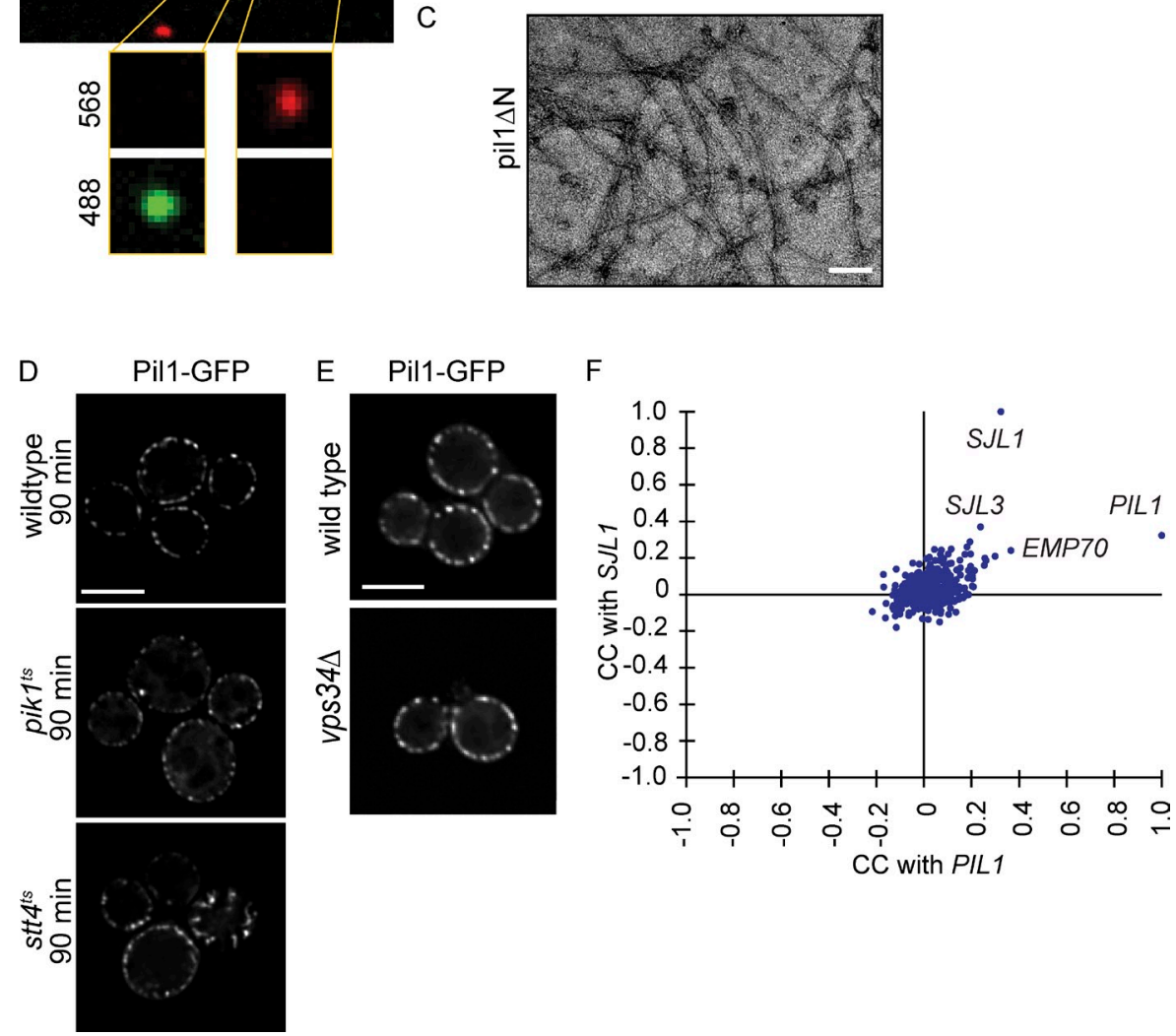

Figure S1. Eisosome proteins form highly stable filaments of variable diameter, and their localization is specifically dependent on plasma membrane PI (4,5) $\mathbf{P}_{2}$. (A) Recombinant Pil 1 was mutated by single-amino acid replacement to pil 1 (A254C) and coupled to either an Alexa Fluor 488 (denoted pil 1 $\mathrm{A} 488$ ) or 568 (denoted pil 1-A568) fluorophore, incubated for $3 \mathrm{~h}$, and imaged by fluorescence microscopy. Mutation and labeling had no influence on localization of Pill or Lspl in eisosomes or the assembly in vitro or in vivo, respectively (not depicted). Recombinant labeled pill (A254C) and lsp 1 (A254C) formed foci when visualized alone by fluorescence microscopy (not depicted). Bar, 5 mm. (B) Number of continuous stretches of 30- or 32-nm diameter plotted versus (y axis) the length of the corresponding Lsp 1 filament (in the absence of the membrane). Most filaments exhibit stretches of both narrow and wide diameter. $\mathrm{N}$ represents the number of filaments used. (C) Negative staining and EM of recombinant pil $1 \Delta \mathrm{N}$. In the absence of its $\mathrm{N}$-terminal segment, the pil $1 \Delta \mathrm{N}$ does not form thick helices anymore, only thin filaments. Bar, $100 \mathrm{~nm}$. (D) Localization of Pill-GFP in wild-type, pik $1^{\text {ts }}$, and stt $4^{\text {ts }}$ cells after incubation at the restrictive temperature for $90 \mathrm{~min}$ and assessed by deconvolution fluorescence microscopy. (E) Localization of Pil 1-GFP in wild-type and vps34 cells, as assessed by deconvolution fluorescence microscopy. (D and E). Bars, $5 \mu \mathrm{m}$. (F) SJL1, encoding the $\mathrm{PI}(4,5) \mathrm{P}_{2}$ phosphatase, has the most similar genetic signature to PIL1, indicating similar gene function (Aguilar et al., 2010). CC, correlation of correlations. 
A

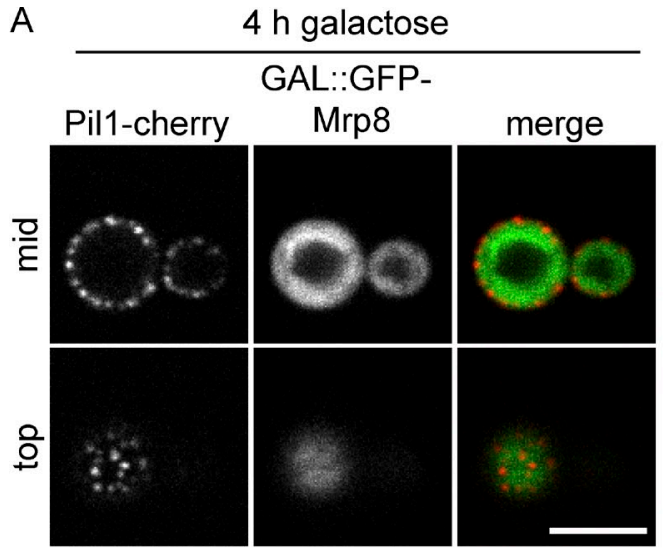

C

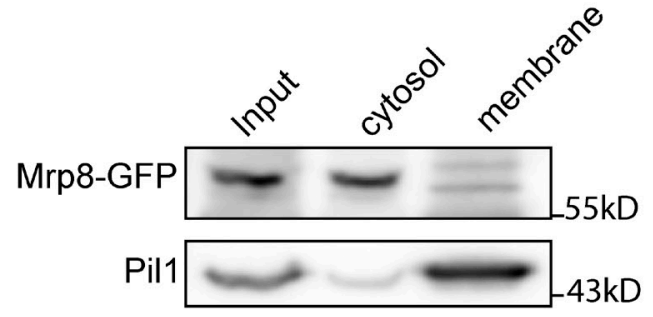

Pma1-GFP

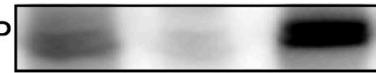

$130 \mathrm{kD}$
B
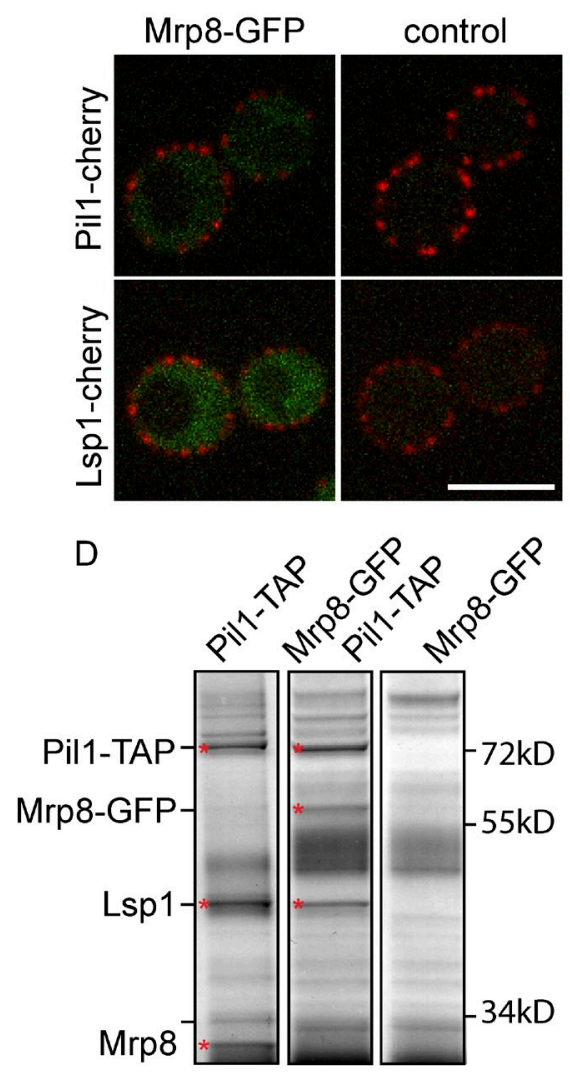

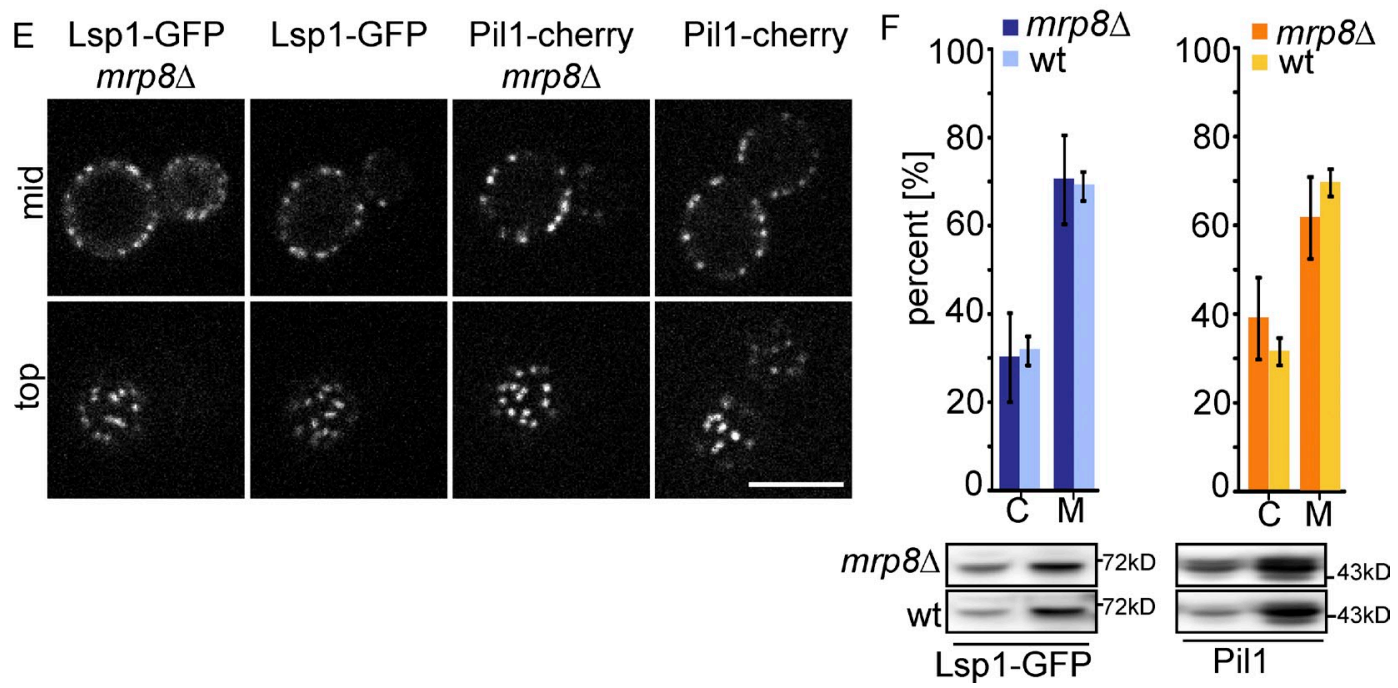

Figure S2. Mrp8 does not localize in eisosomes and is not required for normal eisosomes. (A) N-terminally GFP-tagged Mrp8 was expressed from a GAL promoter. Confocal mid and top sections show GFP-Mrp8 localizing in the cytoplasm, distinct from Pill-cherry-labeled eisosomes. (B) Fluorescence microscopy of C-terminally tagged Mrp8-GFP and expression under its endogenous promoter. Mrp8-GFP localizes in the cytoplasm, distinct from eisosomes marked by Pil 1-cherry or Lsp 1-cherry. (C) Mrp8-GFP fractionates as a cytosolic protein. Western blot analysis of yeast cell lysate after crude membrane versus cytosol fractionation. The majority of Mrp8 fractionates as a cytoplasmic protein, whereas Pill behaves as a membrane protein, similar to Pma 1 GFP. (D) Coomassie blue-stained SDS-PAGE of Pil 1-TAP immunoprecipitation experiments. Pil 1-TAP (Pill fused to a tandem affinity purification tag) purifies with Lsp1 as well as with either Mrp8 or Mrp8-GFP. For better visibility, indicated protein bands are marked by red asterisks. (E) mrp8 $\Delta$ deletion has no effect on Lsp 1-GFP and Pill-cherry localization. Confocal mid and top sections are shown. (A, B, and E) Bars, 5 um. (F) Quantification of cytoplasmic (marked as C) versus membrane (marked as M) signal of Lsp1-GFP mrp8s cells by Western blotting using antibodies recognizing Lsp 1-GFP as well as Pill. Quantification of band intensities shows no significant difference between mrp8s and control cells. Error bars represent three independent measurements. wt, wild type. 


\section{A}
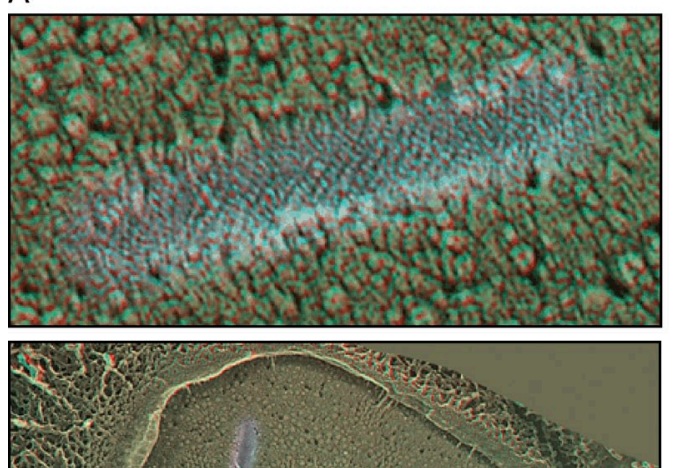

B

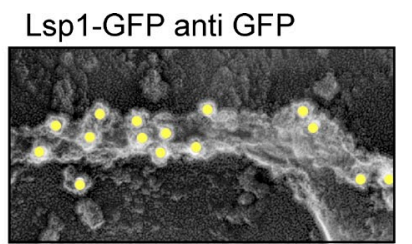

Pil1 anti Pil1

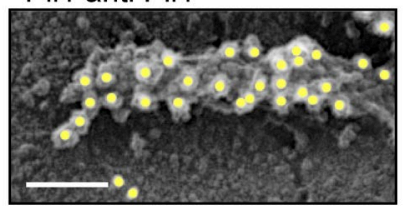

C

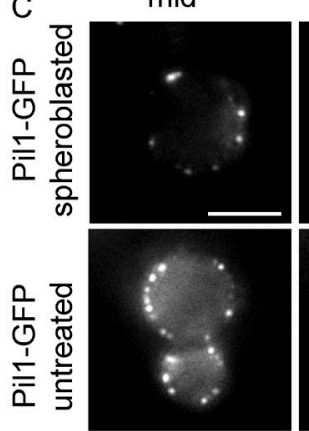

top

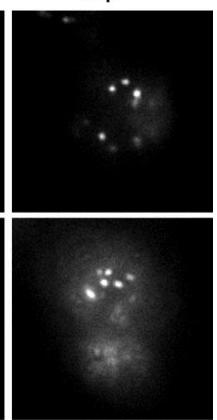

D
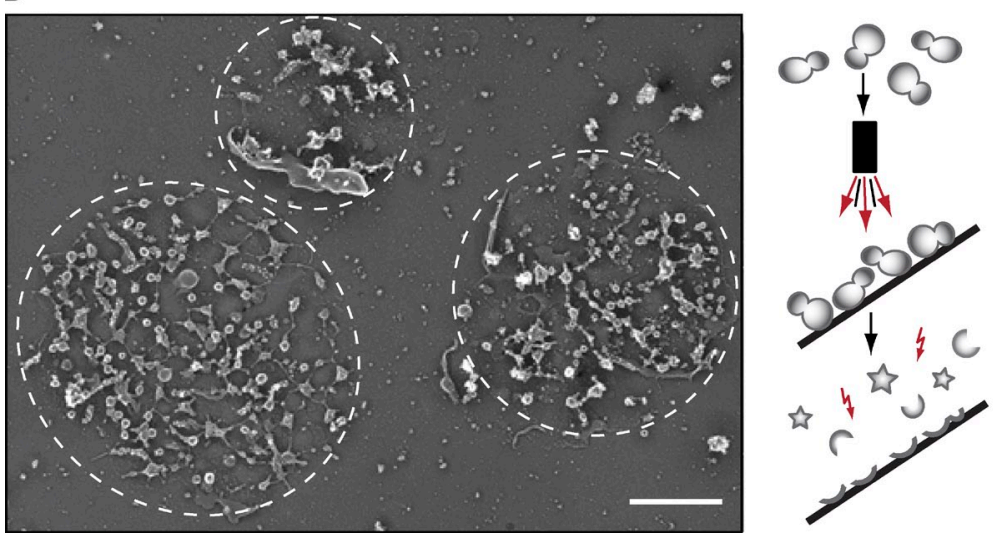

Figure S3. The yeast plasma membrane exhibits abundant furrows that are PIL1 dependent. (A) 3D anaglyphs show freeze-fracture views of the yeast plasma membrane (P-face). The membrane exhibits abundant furrows, showing a distinct striation pattern (red cyan 3D glasses are recommended for 3D view). The boxed area is magnified in the top image. Bar, $500 \mathrm{~nm}$. (B) Immunolabeling of plasma membranes of cells expressing Lsp 1-GFP using anti-GFP antibodies or wild-type cells using anti Pill antibodies. Yellow circles highlight 18-nm gold particles for better visibility. Bar, $100 \mathrm{~nm}$. (C) Fluorescence microscopy of Pill-GFP in spheroblasted cells treated with zymolyase (top panels) and control cells (bottom panels). Bar, $5 \mu \mathrm{m}$. (D) Schematic presentation of workflow for unroofing yeast cells and an overview of plasma membrane fragments generated by this procedure. Magnified selections from such images are shown in Fig. 8. Encircled regions represent areas of plasma membrane fragments generated by this procedure. Bar, 1 pm. 


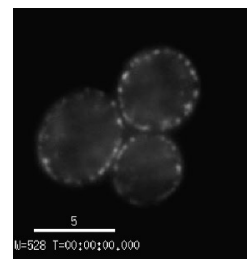

Video 1. Time-lapse imaging of eisosomes at time point $t=0$ min during Mss4 inactivation. Single-focal plane videos of $m s s 4^{t s}$ cells expressing Pill-GFP at the permissive temperature $\left(24^{\circ} \mathrm{C}\right)$. Images were captured using a DeltaVision system equipped with an IX-71 microscope, a 1.35 NA 100x Olympus objective, and a CoolSNAP HQ camera. Frames were taken every $0.5 \mathrm{~s}$. T represents time, whereas $W$ represents the emission wavelength $(528 \mathrm{~nm})$ for the FITC filter used in the video. Bar, $5 \mu \mathrm{m}$.

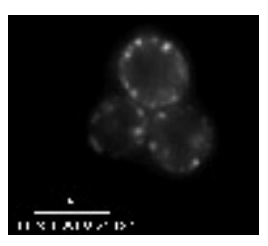

Video 2. Time-lapse imaging of eisosomes after $\mathbf{3 0} \mathrm{min}$ of Mss 4 inactivation. Single-focal plane videos of $m s s 4^{\text {ts }}$ cells expressing Pil 1-GFP after 30 min of a temperature shift to the restrictive temperature $\left(37^{\circ} \mathrm{C}\right)$. Images were captured using a DeltaVision system equipped with an IX-71 microscope, a 1.35 NA 100x Olympus objective, and a CoolSNAP HQ camera. Frames were taken every $0.5 \mathrm{~s}$. T represents time, whereas $W$ represents the emission wavelength $(528 \mathrm{~nm})$ for the FITC filter used in the video. Bar, 5 rm.

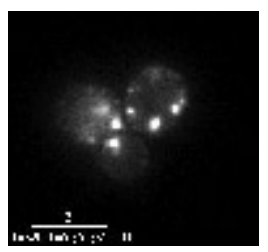

Video 3. Time-lapse imaging of eisosomes after $\mathbf{6 0}$ min of Mss 4 inactivation. Single-focal plane videos of mss $4^{\text {ts }}$ cells expressing Pill-GFP after $60 \mathrm{~min}$ of a temperature shift to the restrictive temperature $\left(37^{\circ} \mathrm{C}\right)$. Images were captured using a DeltaVision system equipped with an IX-71 microscope, a 1.35 NA 100x Olympus objective, and a CoolSNAP HQ camera. Frames were taken every $0.5 \mathrm{~s}$. T represents time, whereas $W$ represents the emission wavelength $(528 \mathrm{~nm})$ for the FITC filter used in the video. Bar, 5 um.

Table S1. Yeast strains used in this study

\begin{tabular}{|c|c|c|}
\hline Strain & Genotype & Reference \\
\hline TWY138 & MATa ura3 trp 1 leu2 his3 ade2 can $1-100$ & Walther et al., 2006 \\
\hline TWY1512 & MATa ura3 trp 1 leu2 his3 ade2 can 1-100 Isp 14::HIS Pill-GFP::KAN & This study \\
\hline TWY1952 & MATa ura3 trp 1 leu2 his3 ade2 can 1-100 Lsp 1-GFP::HIS pil14::NAT & This study \\
\hline SEY6210 & MAT_leu2-3, 112 ura3-52 his3-_200 trp 1-_901 lys2-801 suc2-_9 & Robinson et al., 1988 \\
\hline TWY770 & SEY6210; Pill-GFP::KAN & This study \\
\hline AAY202 & SEY6210; mss4_::HIS3MX6 carrying Ycplacmss4-102 (LEU2 CEN6 mss4-102) & Stefan et al., 2002 \\
\hline TWY764 & AAY202; Pill-GFP::URA & This study \\
\hline TWY2260 & AAY202; Pill-GFP::URA, Sur7-mars::Nat & This study \\
\hline TWY2491 & MATa ura $3 \operatorname{trp} 1$ leu2 his3 ade2 can 1-100 Lsp 1-GFP::HIS mrp8s::HPH & This study \\
\hline TWY2495 & MAT $\alpha$ ura 3 trp 1 leu2 his3 ade2 can 1-100 Pill-cherry::HIS mrp8s::HPH & This study \\
\hline TWY2490 & MAT $\alpha$ ura3 trp 1 leu2 his3 ade2 can 1-100 Pill-cherry::HIS NAT::GAL::GFP-Mrp8 & This study \\
\hline TWY2489 & MATo ura3 trp 1 leu2 his3 ade2 can 1-100 Pil1-cherry::HIS Mrp8-GFP::HPH & This study \\
\hline TWY2492 & MATa ura3 trp 1 leu2 his3 ade2 can 1-100 Mrp8-GFP::HPH & This study \\
\hline TWY958 & MAT $\alpha$ ura3 trp 1 leu2 his3 ade2 can 1-100 Pma 1-GFP::HIS & Howson et al., 2005 \\
\hline TWY344 & MATa his3_1 leu2_O met15_0 ura3_O Pill-TAP::HIS & Howson et al., 2005 \\
\hline TWY2496 & MATa his3_1 leu2_0 met15_0 ura3_0 Pil1-TAP::HIS Mrp8-GFP::HPH & This study \\
\hline
\end{tabular}

Underlined text represents standard genetic nomenclature denoting MAT $\alpha \alpha$ and MATa as standard names for mating type loci.

Table S2. Dataset for helical reconstruction

\begin{tabular}{lccc}
\hline Sample & Micrographs & Filaments & Segments \\
\hline Lspl & 218 & 2,515 & 55,500 \\
Lspl-PI $(4,5) P_{2}$ & 105 & 158 & 4,794 \\
Pill-PI(4,5)P 2 & 123 & 638 & 15,303 \\
\hline
\end{tabular}


Table S3. Data collection and reconstruction statistics for helical reconstruction

\begin{tabular}{|c|c|c|c|c|c|c|c|}
\hline Dataset & $\begin{array}{c}\text { Principal layer line } \\
\text { orders }\end{array}$ & Diameter & Turn & Rise & Symmetry & No. of segments & Resolution \\
\hline & & $n m$ & Degrees & $\AA$ & & & $\AA$ \\
\hline \multicolumn{8}{|l|}{ Lspl } \\
\hline$\# 1$ & $7,-13$ & 30 & -53.1 & 5.5 & $\mathrm{Cl}$ & 34,308 & 25 \\
\hline \#2 & $8,-13$ & 32 & -136.0 & 5.2 & $\mathrm{Cl}$ & 9,210 & 29 \\
\hline \multicolumn{8}{|c|}{ Lsp 1-liposome } \\
\hline$\# 1$ & $9,-13$ & 34 & -80.8 & 4.9 & $\mathrm{Cl}$ & 1,135 & 31 \\
\hline \#2 & $8,-14$ & 33 & -47.5 & 10.4 & $\mathrm{C} 2$ & 1,725 & $n / a$ \\
\hline \#3 & $8,-12$ & 32 & -49.7 & 22.0 & $\mathrm{C} 4$ & 964 & $n / a$ \\
\hline \multicolumn{8}{|c|}{ Pill-liposome } \\
\hline$\# 1$ & $7,-15$ & 34 & 49.4 & 5.1 & $\mathrm{Cl}$ & 3,833 & 29 \\
\hline \#2 & $8,-14$ & 34 & -48.2 & 10.1 & $\mathrm{C} 2$ & 2,696 & 35 \\
\hline \#3 & $8,-15$ & 36 & -46.6 & 4.8 & $\mathrm{Cl}$ & 1,744 & 35 \\
\hline \#4 & $7,-12$ & 31 & 152.3 & 5.6 & $\mathrm{Cl}$ & 3,917 & $\mathrm{n} / \mathrm{a}$ \\
\hline
\end{tabular}

n/a, not applicable.

\section{References}

Aguilar, P.S., F. Fröhlich, M. Rehman, M. Shales, I. Ulitsky, A. Olivera-Couto, H. Braberg, R. Shamir, P. Walter, M. Mann, et al. 2010. A plasma-membrane E-MAP reveals links of the eisosome with sphingolipid metabolism and endosomal trafficking. Nat. Struct. Mol. Biol. 17:901-908. http://dx.doi.org/10.1038/nsmb.1829

Howson, R., W.K. Huh, S. Ghaemmaghami, J.V. Falvo, K. Bower, A. Belle, N. Dephoure, D.D. Wykoff, J.S. Weissman, and E.K. O'Shea. 2005. Construction, verification and experimental use of two epitope-tagged collections of budding yeast strains. Comp. Funct. Genomics. 6:2-16. http://dx.doi.org/10.1002/cfg.449

Robinson, J.S., D.J. Klionsky, L.M. Banta, and S.D. Emr. 1988. Protein sorting in Saccharomyces cerevisiae: Isolation of mutants defective in the delivery and processing of multiple vacuolar hydrolases. Mol. Cell. Biol. 8:4936-4948.

Stefan, C.J., A. Audhya, and S.D. Emr. 2002. The yeast synaptojanin-like proteins control the cellular distribution of phosphatidylinositol (4,5)-bisphosphate. Mol. Biol. Cell. 13:542-557. http://dx.doi.org/10.1091/mbc.01-10-0476

Walther, T.C., J.H. Brickner, P.S. Aguilar, S. Bernales, C. Pantoja, and P. Walter. 2006. Eisosomes mark static sites of endocytosis. Nature. 439:998-1003. http://dx.doi. org/10.1038/nature04472 